"Nem a kép tárgya a fontos, hanem a szellemi valóság, amely általa testet öltött." Bernáth Aurél 


\title{
Exogen and endogen events trigger complex interactions in the melanoma microenvironment
}

\author{
PhD Thesis \\ Edina Gyukity-Sebestyén \\ Supervisor: \\ Krisztina Buzás, PhD
}

Department of Immunology, Faculty of Medicine, Faculty of Science and Informatics, University of Szeged

Biological Research Centre, Institute of Biochemistry
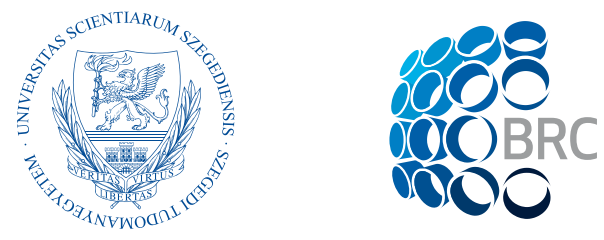

Doctoral School of Interdisciplinary Medicine,

Faculty of Medicine, University of Szeged

Szeged

2020 


\section{The dissertation is based on the following publications:}

I. Gyukity-Sebestyén E, Harmati M, Dobra G, Németh IB, Mihály J, Zvara Á, HunyadiGulyás É, Katona R, Nagy I, Horváth P, Bálind Á, Szkalisity Á, Kovács M, Pankotai T, Borsos B, Erdélyi M, Szegletes Z, Veréb ZJ, Buzás EI, Kemény L, Bíró T, Buzás K. Melanoma-Derived Exosomes Induce PD-1 Overexpression and Tumor Progression via Mesenchymal Stem Cell Oncogenic Reprogramming. Front Immunol. 10, 2459. doi: 10.3389/fimmu.2019.02459 (2019)

IF 5.085; Q1

II. Buzás K, Marton A, Vizler C, Gyukity-Sebestyén E, Harmati M, Nagy K, Zvara Á, Katona RL, Tubak V, Endrész V, Németh IB, Oláh J, Vígh L, Bíró T, Kemény L. Bacterial Sepsis Increases Survival in Metastatic Melanoma: Chlamydophila Pneumoniae Induces Macrophage Polarization and Tumor Regression. J Invest Dermatol. 136, 862-5. doi: 10.1016/j.jid.2015.12.032 (2016)

IF 6.287; Q1

\section{Further publications}

I. Dobra G, Bukva M, Szabo Z, Bruszel B, Harmati M, Gyukity-Sebestyen E, Jenei A, Szucs M, Horvath P, Biro T, Klekner A, Buzas K. Small Extracellular Vesicles Isolated from Serum May Serve as Signal-Enhancers for the Monitoring of CNS Tumors. Int J Mol Sci. 21(15). doi: 10.3390/ijms21155359 (2020)

IF 4.556; Q2

II. Harmati M, Gyukity-Sebestyen E, Dobra G, Janovak L, Dekany I, Saydam O, HunyadiGulyas E, Nagy I, Farkas A, Pankotai T, Ujfaludi Z, Horvath P, Piccinini F, Kovacs M, Biro T, Buzas K. Small extracellular vesicles convey the stress-induced adaptive responses of melanoma cells. Sci Rep. 9;15329. doi: 10.1038/s41598-019-51778-6 (2019)

IF 3.998; Q1

III. Farkas B, Zsedenyi A, Gyukity-Sebestyen E, Romano I, Nagy K, Diaspro A, Brandi F, Buzas K and Beke S. Excimer Laser-produced Biodegradable Photopolymer Scaffolds Do Not Induce Immune Rejection In Vivo. JLMN-Journal of Laser Micro/Nanoengineering 10, No. 1, doi: 10.2961/jlmn.2015.01.0002 (2015) IF 0.66; Q2

IV. Zsedenyi A, Farkas B, Abdelrasoul GN, Romano I, Gyukity-Sebestyen E, Nagy K, Harmati M, Dobra G, Kormondi S, Decsi G, Nemeth IB, Diaspro A, Brandi F, Beke S, Buzas K. Gold nanoparticle-filled biodegradable photopolymer scaffolds induced muscle remodeling: in vitro and in vivo findings. Mater Sci Eng C Mater Biol Appl. 72,625-630. doi: 10.1016/j.msec.2016.11.124 (2016)

IF 5.08; Q1 
V. Harmati M, Tarnai Z, Decsi G, Kormondi S, Szegletes Z, Janovak L, Dekany I, Saydam O, Gyukity-Sebestyen E, Dobra G, Nagy I, Nagy K, Buzas K. Stressors alter intercellular communication and exosome profile of nasopharyngeal carcinoma cells. J Oral Pathol Med. 46,259-266. doi: 10.1111/jop.12486 (2017)

IF 2.237; Q2

VI. Harmati M, Gyukity-Sebestyen E, Dobra G, Terhes G, Urban E, Decsi G, Mimica-Dukić N, Lesjak M, Simin N, Pap B, Nemeth IB, Buzas K. Binary mixture of Satureja hortensis and Origanum vulgare subsp. hirtum essential oils: in vivo therapeutic efficiency against Helicobacter pylori infection. Helicobacter. 22,e12350. doi: 10.1111/hel.12350 (2017)

IF 4.123; Q1

VII. Dobó J, Harmat V, Beinrohr L, Sebestyén E, Závodszky P, Gál P. MASP-1, a promiscuous complement protease: structure of its catalytic region reveals the basis of its broad specificity. J Immunol. 183,1207-14. doi: 10.4049/jimmunol.0901141 (2009) IF 5.0; Q1

VIII. Dobó J, Harmat V, Sebestyén E, Beinrohr L, Závodszky P, Gál P. Purification, crystallization and preliminary X-ray analysis of human mannose-binding lectinassociated serine protease-1 (MASP-1) catalytic region. Acta Cryst. 64,781-784. doi: 10.1107/S174430910802294X (2008)

IF 0.91; Q3 


\section{CONTENTS}

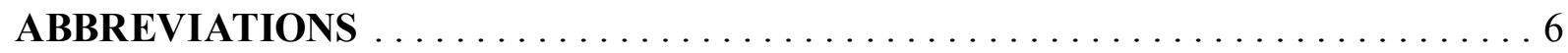

1. INTRODUCTION $\ldots \ldots \ldots \ldots \ldots \ldots \ldots \ldots \ldots \ldots \ldots \ldots \ldots \ldots \ldots$

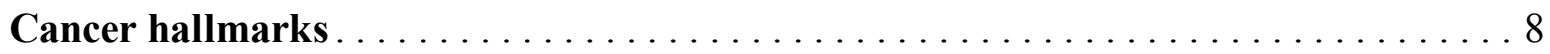

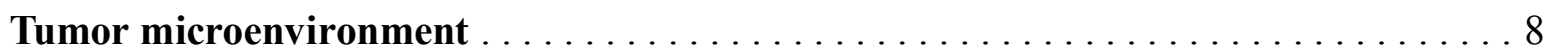

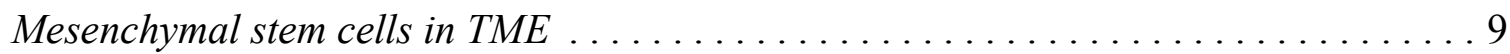

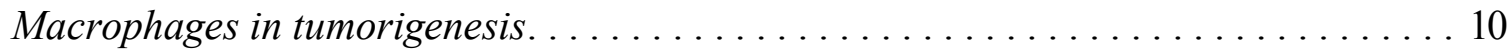

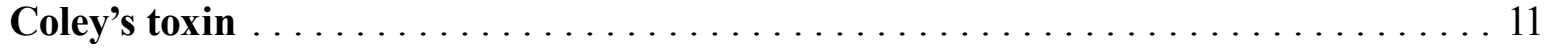

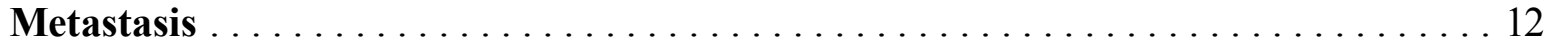

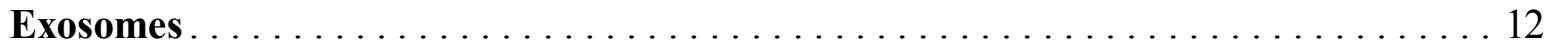

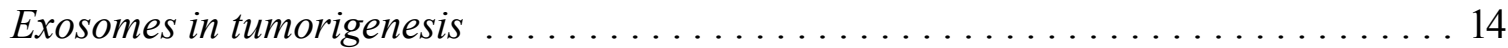

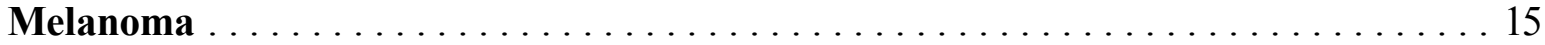

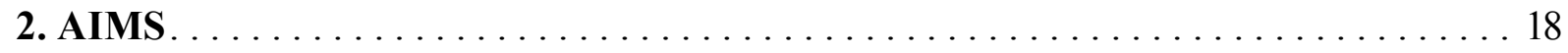

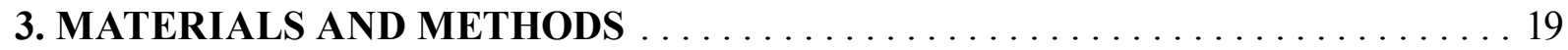

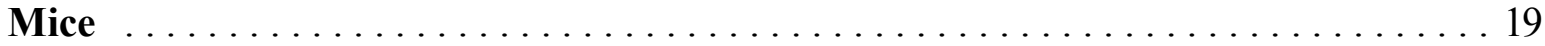

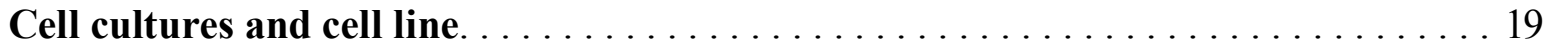

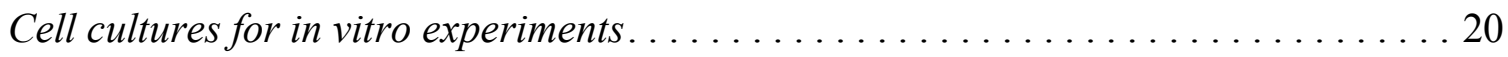

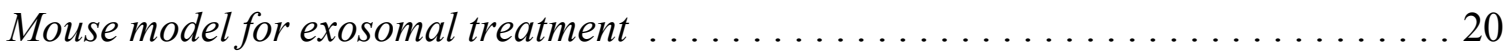

The mouse model for Chlamydia treatment . . . . . . . . . . . . . . . . . . . 21

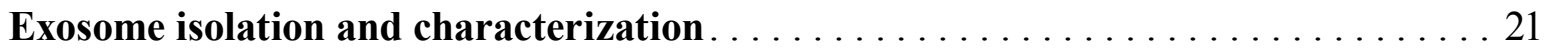

Determination of shape and size of the isolated vesicles . . . . . . . . . . . 22

Identification of exosomal proteins by mass spectrometry . . . . . . . . . . . 22

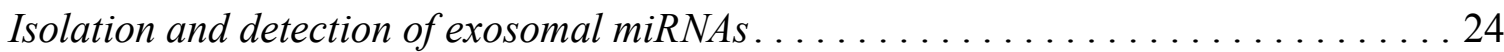

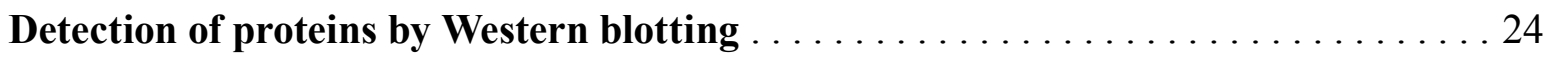

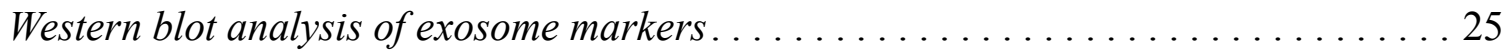

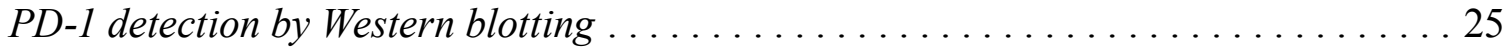

Cytokine and chemokine detection by proteome profiling $\ldots \ldots \ldots \ldots \ldots \ldots$ 


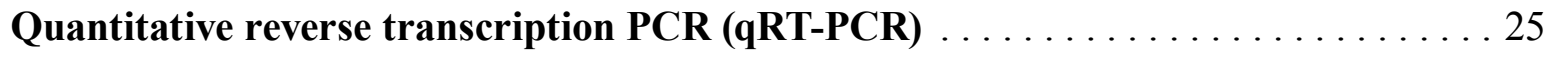

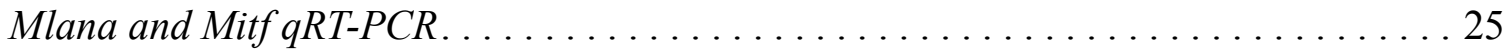

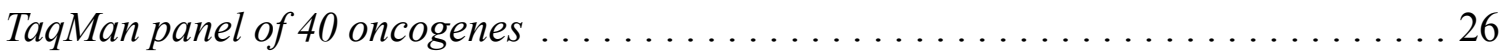

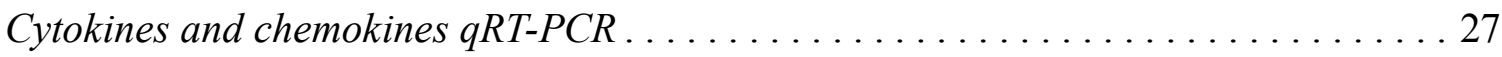

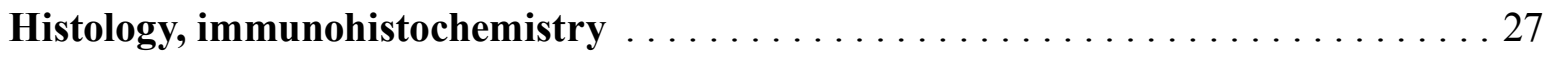

Visualization of labeled exosome internalization in MSCs. . . . . . . . . . . 27

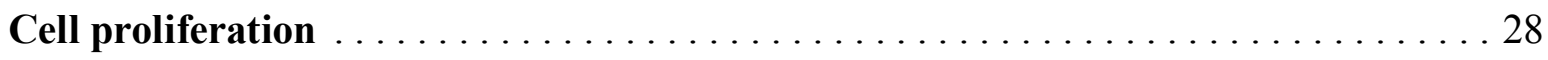

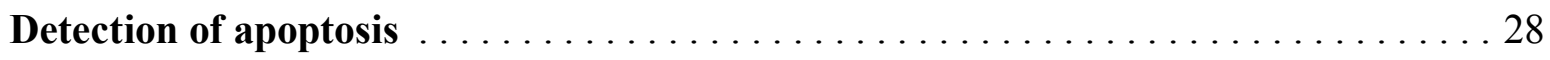

PD-1 and MLANA detection by immunofluorescence microscopy . . . . . . . . . 29

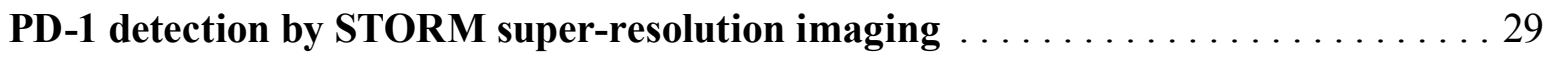

Network representation by Ingenuity Pathway Analysis $\ldots \ldots \ldots \ldots \ldots \ldots \ldots \ldots$

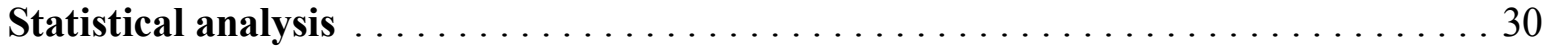

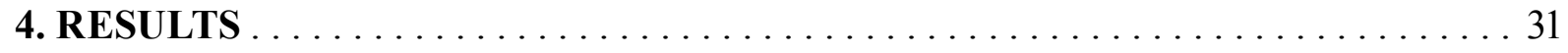

Isolated vesicles show exosomal properties $\ldots \ldots \ldots \ldots \ldots \ldots \ldots \ldots \ldots \ldots \ldots \ldots \ldots \ldots$

Tumor exosome exposure resulted in oncogenic reprogramming of MSCs in vitro . . 32

B16F1 exosomes augment in vivo tumorigenesis and tumor progression . . . . . . . 39

Melanoma-derived exosomes promote tumorigenic and cell survival signaling

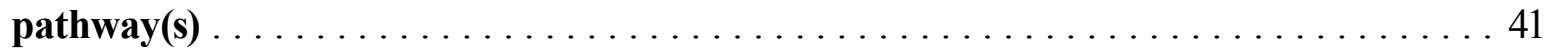

Treatment of immunocompetent mice with $C$. pneumoniae reduces

the number of lung metastases and increases survival $\ldots \ldots \ldots \ldots \ldots \ldots \ldots \ldots$

C. pneumoniae treatment induces tumor regression and immune cell infiltration . . 44

The $C$. pneumoniae treatment induces the macrophage differentiation shift toward an M1 phenotype . . . . . . . . . . . . . . . . . . . . . . . . . . . . . . . 47

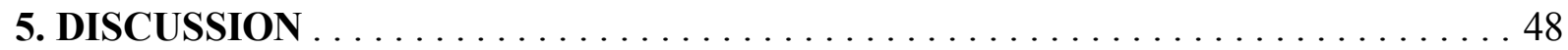

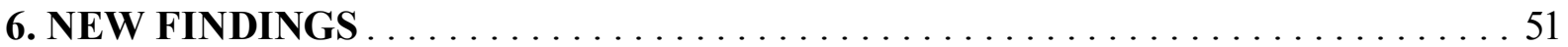

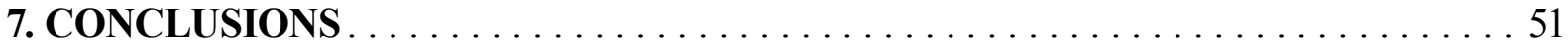

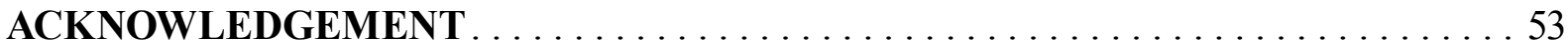

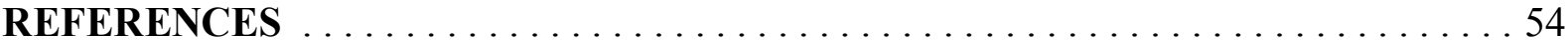

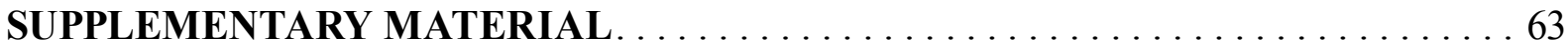




\section{ABBREVIATIONS}

AKT Protein kinase B

ALCAM1 Activated leukocyte cell adhesion molecules

BAX Bcl-2-associated $X$ protein

BCL2 B-cell lymphoma

C.pneumoniae Chlamydia pneumoniae

C.pn. Chlamydia pneumoniae

CSF Colony stimulating factor

ECM Extracellular matrix

ETS E26 transcription factor

EVs Extracellular vesicles

HRP Horseradish peroxidase

HSP70 70kDa heat shock protein

IL Interleukin

IPA Ingenuity Pathway Analysis

ISEV International Society for Extracellular Vesicles

ITG Integrin

JAK Janus kinase

KIT Mast/stem cell growth factor receptor (SCFR)

KITL KIT ligand

LC-MS/MS Liquid Chromatography - Tandem Mass Spectrometry

MAPK Mitogen-activated protein kinase

MET Receptor tyrosine kinase 
MHC Major histocompatibility complex

miRNA microRNA

MISEV2018 Minimal Information for Studies of Extracellular Vesicles 2018

MITF Microphthalmia-associated transcription factor

MLANA Melanoma antigen recognized by T-cells

mRNA messenger RNA

MSC Mesenchymal stem cell

mTOR Mammalian target of rapamycin

MVB Multivesicular body

NSG NOD/Scid IL2rg null (immunodeficient mice)

PD-1 Programmed cell death protein 1

PD-L1 Programmed cell death protein 1 ligand

PI3K Phosphoinositide 3-kinase

PMN Pre-metastatic niche

qRT-PCR Quantitative reverse transcription PCR

RAB Ras-associated binding protein

RAF1 Proto-oncogene serine/threonine-protein kinase

STAT Signal-transducer and activator of transcription

STORM Stochastic optical reconstruction microscopy, super-resolution imaging

TAM Tumor-associated macrophage

TGF Transforming growth factor

TLR Toll-like receptor

TME Tumor microenvironment

TNF- $\alpha \quad$ Tumor necrosis factor- $\alpha$ 


\section{INTRODUCTION}

\section{Cancer hallmarks}

Hanahan and Weinberg were the first to propose the six hallmarks of cancer, which provided a useful conceptual structure for understanding the complex biology of cancer (1). These hallmarks are proliferative signaling, evading growth suppressors, resisting cell death, enabling replicative immortality, inducing angiogenesis, activating invasion and metastasis. Genome instability underlies these hallmarks, which generates the genetic diversity that accelerates their acquisition, and inflammation supporting multiple hallmark function. Theoretical progress in the last decade has added additional hallmarks potentially characteristic of all cancers which transform cellular metabolism and evade the immune system (Figure 1) (2).

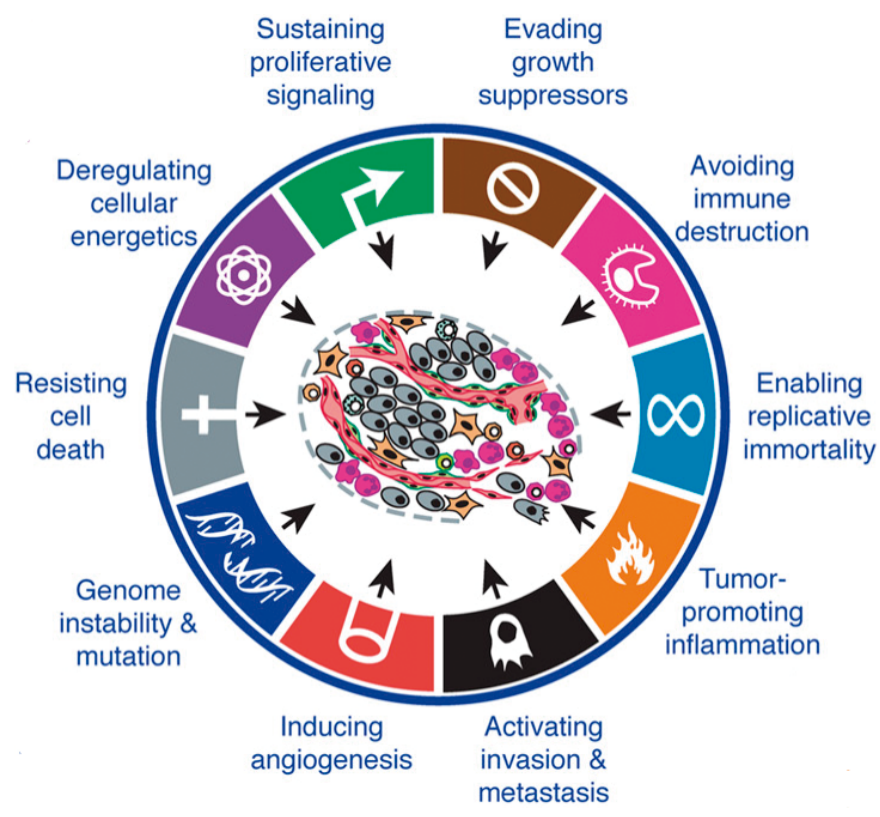

Figure 1. The hallmarks of cancer.

\section{Tumor microenvironment}

Made up of tumor cells and stroma, the tumor microenvironment (TME) is a dynamic milieu, which is continuously evolving and includes blood vessels, lymph vessels, extracellular matrix (ECM), stromal cells, immune/inflammatory cells, secreted proteins, RNAs and small 
organelles. Cancer cells manipulate their microenvironment through the secretion of various cytokines, chemokines, growth factors and extracellular vesicles (EVs). The development of a supporting niche is critical for the continuous and uncontrolled growth of cancer. Meanwhile, tumor cells persistently shape their microenvironment, thereby establishing an abnormal ambience (3). Stromal cells of the TME can be educated to promote cancer development and progression. Whereas normal fibroblasts do not show potent tumorigenesis, tumorassociated fibroblasts promote tumorigenesis. These properties indicate that the tumorigenesis of inflammatory fibroblasts is due to the influence of cancer cells $(4,5)$. Recruitment of monocytes is one of the primary events in tumor development, and the functional plasticity of macrophages, the phenotype of tumor-associated-macrophage (TAM) is strongly influenced by microenvironmental factors present within the developing tumor, which appear to promote their pro-tumoral function (6). Tumor-stroma interactions modulate the microenvironment to be more permissive towards cancer cells (7). Tumor cell hierarchies, as well as multiple cellular elements in the microenvironment, co-evolve during the process of carcinogenesis (8).

\section{Mesenchymal stem cells in TME}

Mesenchymal stem cells (MSCs) are a major component of the TME. The MSCs home to a cancer microenvironment in response to various cytokines, chemokines and EVs secreted by cancer cells. MSCs are a population of adult multipotent cells capable of self-renewal; they differentiate into many different cells of mesodermal origin, such as osteoblasts, chondrocytes and adipocytes (9).

Under tumoral influence, MSCs have been shown to differentiate into multiple tumor-associated cells types. Additionally, MSCs can maintain their phenotype and alter their cytokine secretion profile. Tumor-associated MSCs secrete increased levels of cytokines, chemokines, growth factors, and other small molecules, which interact with the tumor cells and the microenvironment. Conflicting data and concepts on antitumor and tumor-promoting roles of MSCs have been reported. Indeed, cellular fate could depend on the cancer type and also on the status of the affected MSCs. Activated MSCs can secrete pro-angiogenic soluble factors and are able to differentiate to vessel wall-associated cells (10) or to cancer-associated fibroblasts $(11,12)$. Most reports suggest MSCs have tumor-promoting roles (13). MSC can prompt osteosarcoma progression through interleukin (IL)-6 secretion induced by tumor exosomes (14). The bone marrow-derived cells enhance the metastatic behavior of primary malignant melanoma via permanent education by tumor cell-derived exosomes (15). On the other hand, the MSCs similar to the pro- and antitumor phenotypes described for macrophages can either stimulate or inhibit tumor progression depending on the environmental cue. For instance, it has been shown that MSCs can inhibit the malignant phenotypes of human liver cancer cell lines, in a number of 
ways, including proliferation, colony-forming ability, and oncogene expression both in vitro and in vivo (16).

An enhanced understanding of crosstalk between MSCs and tumor cells may reveal better targets for new therapies.

\section{Macrophages in tumorigenesis}

Macrophages are immune cells derived from the myeloid lineage, and they play an important role in tissue development, homeostasis and immune surveillance (17). TAMs are among the most abundant immune cells in the TME, and they play a key role in oncogenic processes of tumor proliferation, angiogenesis and metastasis. Mirroring the paradigm of Th1/Th2 lymphocytes, macrophages can be subdivided into two polarized subsets; classically activated macrophages and alternatively activated macrophages, M1 and M2, respectively (18). Macrophages polarize along the type 1 pathway in response to Th1-derived cytokines, such as interferon gamma, colony stimulating factor 2 (CSF2 or GM-CSF) or toll-like receptor (TLR) agonism (19). This induces expression of major histocompatibility complex II (MHC II) and costimulatory molecules (CD80 and CD86) leading to efficient T-cell priming. M1 cells produce nitric oxide (NO) and reactive oxygen species (ROS) associated with microbicidal and tumoricidal activity. Furthermore, M1 polarized macrophages recruit new Th1 cells via chemokines CXCL9 and CXCL10 and produce proinflammatory cytokines, such as tumor necrosis factor alpha (TNF- $\alpha$ ) and IL-1 $\beta$, IL-6, IL-12, and IL-23 (20). M1 infiltration in tumors is associated with a good prognosis in some cancers $(20,22)$. On the other end of the spectrum are M2 macrophages. This immunosuppressive state is initiated by Th2-derived cytokines, such as IL-4, IL-10, IL-13, transforming growth factor beta (TGF- $\beta$ ), prostaglandin E2 (PGE2) or colony stimulating factor 1 (CSF1 or M-CSF) $(23,24)$. M2 macrophages lose their antigen presenting capabilities and are involved in tissue remodeling, debris scavenging and immune modulation (17). They support angiogenesis by secretion of adrenomedullin and vascular epithelial growth factors (VEGFs), and express immunosuppressive molecules such as IL-10, programmed death ligand 1 (PD-L1) and TGF- $\beta(25,26)$.

This simplified M1/M2 definition provides a useful framework to classify macrophage function in macrophage biology. However, this definition overlooks the spectrum of trophic/developmental macrophages involved in tissue repair, angiogenesis or bone remodeling, which is found in normal physiology $(27,28)$. During tumor development, at least six distinct TAM populations are found, which often share features of both M1 and M2 types $(19,29)$. This spectrum of activation states is controlled by the cytokine profile of the TME, where different cytokines can integrate and induce a broad and dynamic range of functions (Figure 2). 
M2 macrophage

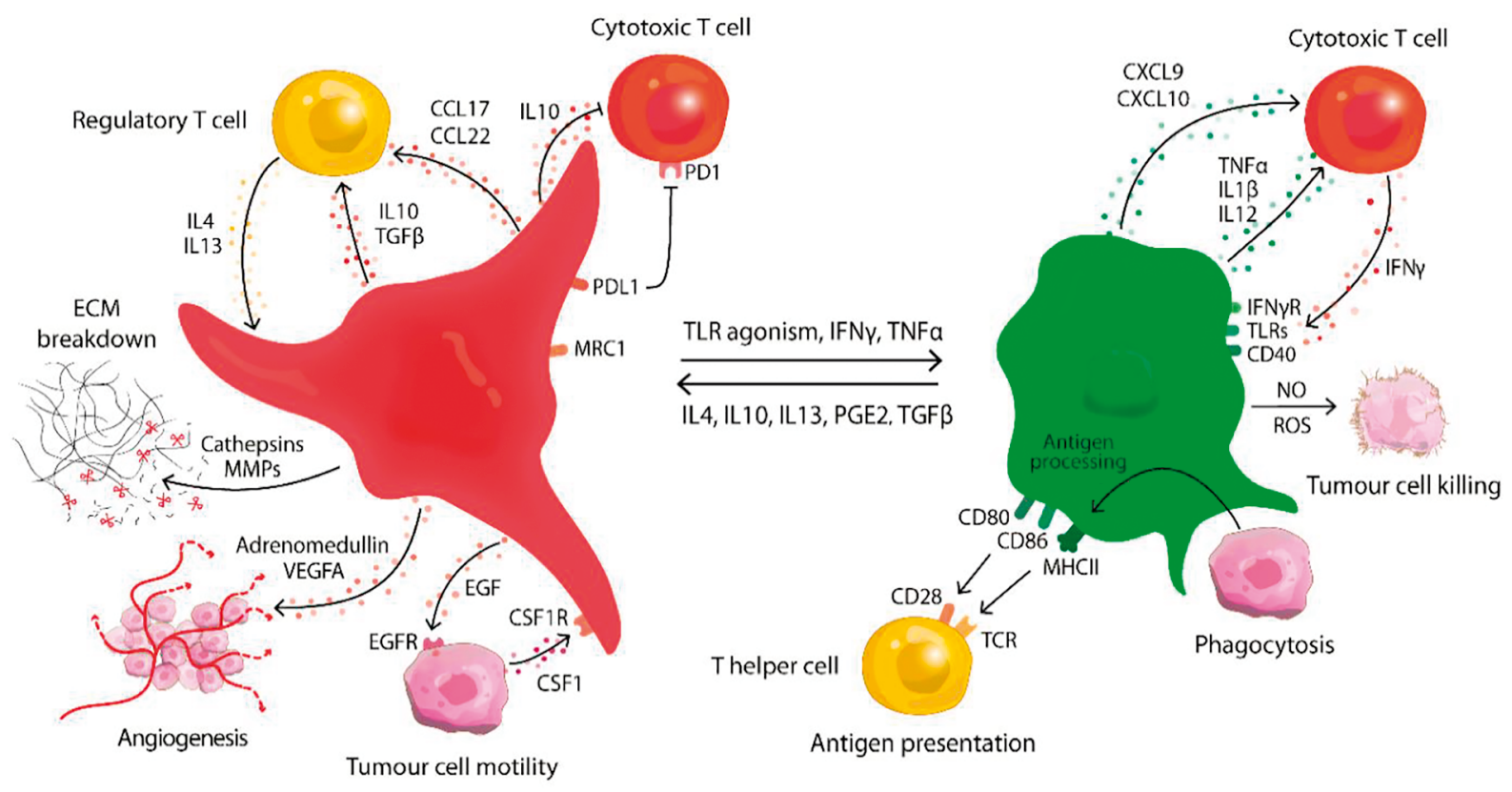

Figure 2. Schematic representation of the functions of M1 and M2 macrophages in tumor development.

Because the tumor mass contains a great number of M2 macrophages, TAMs may be used as a target for cancer treatment. Reducing the number of TAMs or polarizing them towards an M1 phenotype can help destroy cancer cells or impair tumor growth $(30,32)$.

\section{Coley's toxin}

It has been recognized over 100 years ago that cancer patients sometimes recover following bacterial infections (33). In a notable experiment, William B. Coley induced erysipelas by local injection of streptococcal cultures in a patient bearing a lymphoma. Surprisingly, he observed decreased tumor volume and a prompt improvement of the patient's general condition. Based on experiences of the initial study, a large-scale effort to cure cancer patients was initiated using inactivated suspensions of Streptococcus pyogenes and Serratia marcescens (34-39). In 2003, a large clinical study carried out by the European Organization for Research and Treatment of Cancer provided unexpected evidence supporting the positive effect of microbial induction in cancer. Krone B. et al. showed that febrile infections or vaccinations with intracellular pathogens Bacillus Calmette-Guérin or Vaccinia virus in early childhood significantly decrease incidence of melanoma (40). Nevertheless, as these exciting data in most cases were not reproducible, we still lack detailed and convincing explanations of this phenomenon.

Although it is generally accepted that anti-tumor immune effector mechanisms overlap with antibacterial immune responses (41), the exact anti-tumor mechanism induced by microbes is 
not yet understood. With respect to the anti-tumor mechanisms, it is also known that alterations in the polarization patterns of the overall immune system determine the outcome of malignant diseases (42). Key players of the immune responses include macrophages whose M1 type polarization mediates the anti-tumor action of the immune system $(43,44)$.

\section{Metastasis}

Metastasis is the hallmark of cancer that is responsible for the greatest number of cancer-related deaths. In 1889, the English surgeon Stephen Paget proposed the "seed and soil" hypothesis (45). This hypothesis suggested that the metastasis is dependent on the interactions between the cancer cells ('seed') and the host microenvironment ('soil'). The question that first captivated Paget was why tumor cells emerge only as disseminated tumor cells within specific organs. This organ specificity observed in metastasis is known as organotropism and remains one of the most intriguing unanswered questions in cancer research.

In recent years, additional fundamental discoveries have brought fresh insight into our understanding of cancer metastasis, and several novel concepts have been established. Tumors can induce the formation of microenvironments in distant organs that are conducive to the survival and outgrowth of tumor cells before their arrival at these sites (46). These predetermined microenvironments are termed pre-metastatic niches (PMNs) (47). Since the existence of PMNs was first demonstrated, numerous studies have identified various molecules that regulate its stepwise evolution, highlighting the complex molecular and cellular changes that occur in the PMN to support future metastatic tumor growth $(48,49)$. PMNs are the result of the combined systemic effects of tumor-secreted factors and tumor-shed EVs that promote a temporal sequence of events shaping the evolution of PMNs. Although congruent with both theories of Paget, the concept of the PMN is unique as it proposes that the primary tumor preconditions specific organ sites for future metastatic disease (that is, before CTC arrival) via tumor-derived factors (50).

\section{Exosomes}

Exosomes are a subset of extracellular vesicles. Most living cells release an array of EVs, nanoparticles which are $20-1,000 \mathrm{~nm}$ in size. The nomenclature of the different vesicle types depends on their cell of origin, as well as their function and size, and has generated confusion regarding the definition of exosomes. The EV terms used in this expanding area of research encompass exosomes, ectosomes, microvesicles, microparticles, prostasomes, tolerosomes (which induce immunological tolerance to dietary antigens), apoptotic bodies (released by apoptotic cells), and nanovesicles. Cells deliver microRNA (miRNA), messenger RNA (mRNA), proteins, and other biomolecules between intracellular organelles by membrane vesicles, which 
contain receptors to ensure traffic specificity. These membrane vesicles are actively secreted by most cells and exist in most body fluids, including blood, saliva, breast milk and sperm. There are three main types of such membrane vesicles: microparticles, microvesicles $(100-1,000 \mathrm{~nm})$, and exosomes (20-200 nm) (51).

In 2014, the International Society for Extracellular Vesicles (ISEV) proposed Minimal Information for Studies of Extracellular Vesicles ("MISEV") guidelines for the field and updated these guidelines in 2018. MISEV2018 (52) aims to standardize the nomenclature, and includes suggestions for the separation/isolation and characterization protocol of EVs. MISEV2018 suggests the use of the operational term for EV subtypes that refer to physical characteristics, such as size or density, biochemical composition, or releasing conditions. In this study, small EVs (sEVs) were isolated on the basis of their size $(<200 \mathrm{~nm})$, and the isolated vesicles are referred to as "exosomes" by their characterization, shape, and markers.

Exosomes are small, multivesicular body (MVB)-derived EVs constitutively secreted by almost all cell types. During the endocytosis of exosomes, exosomes membrane often incorporates lipid rafts, which contain a variety of tumor-specific receptors and common membrane proteins, such as tetraspanins (e.g. CD9, CD63, CD81), MHC I and II, and adhesion molecules (e.g. integrins, cadherins), and results in the formation of early endosomes. Exosomes show inward budding of the MVB. During this process, numerous proteins (e.g. receptors, ubiquitin-related proteins, heat shock proteins), nucleic acids (e.g. miRNAs, RNAs, DNAs, InRNAs), transcriptional factors, and lipids (e.g. cholesterol, ceramide) can be selectively packed into MVB in a cell type-dependent manner (Figure 3). After early-to-late endosome conversion, late endosomes containing MVB fuse with the plasma membrane to secrete exosomes into the extracellular space by exocytosis, which is mainly controlled by endosome-specific Rab GTPases including Rab11/35, Rab7, and Rab27. The uptake of exosomes by recipient cells can be mediated by direct fusion of exosomes with the cell membrane of the recipients via receptor-ligand interactions or by endocytosis (53). 


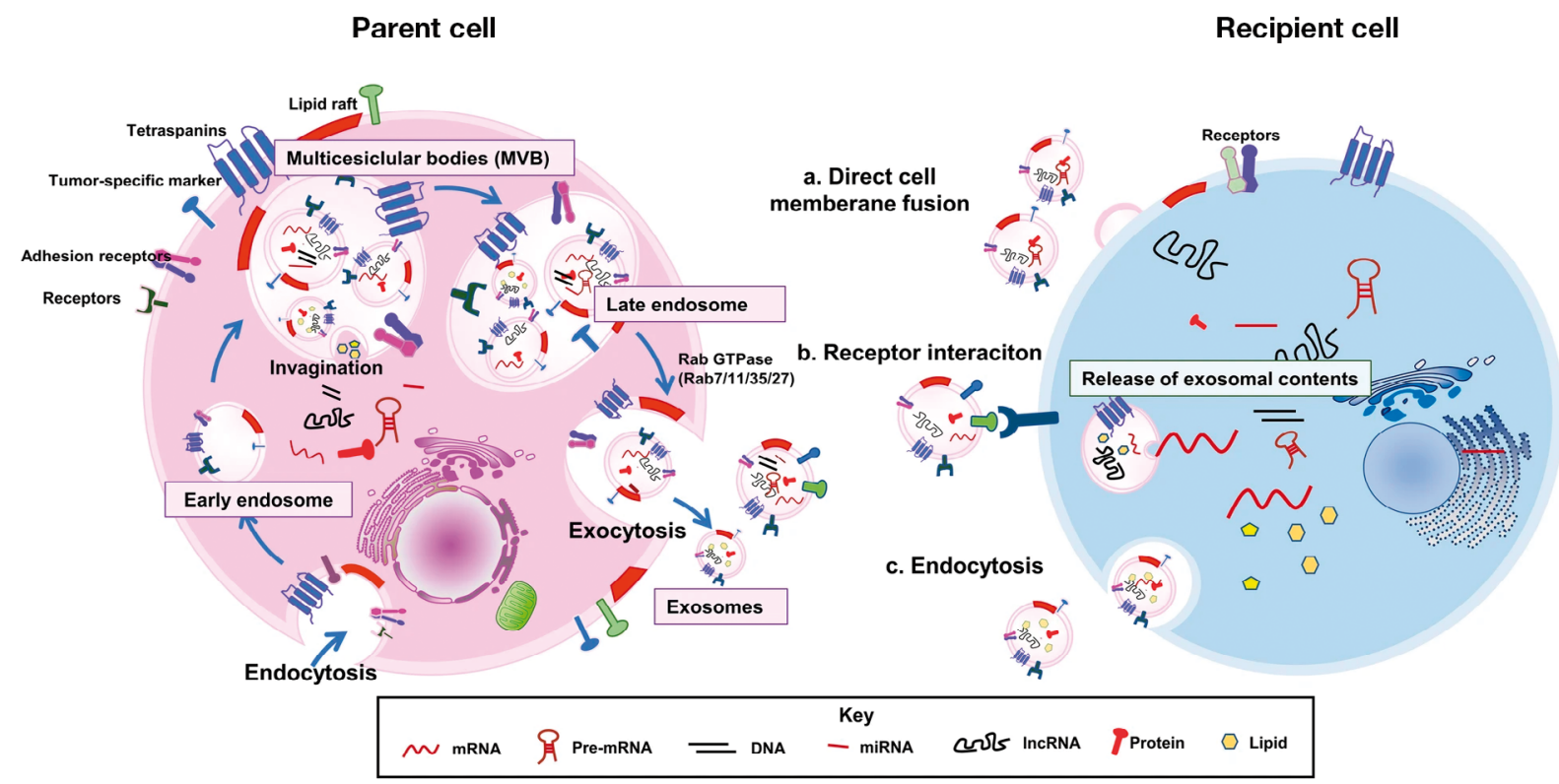

Figure 3. Biogenesis, secretion, and uptake of exosomes.

These exosomal-molecular patterns, as unique entities of the complex intercellular communication, are not independent of the quality or state of the mother cell (54). Indeed, exosomes are claimed to be capable of carrying and conveying tumor-related information and might have a significant role in formation of the tumor microenvironment.

\section{Exosomes in tumorigenesis}

In most cases, primary cancers can be cured by surgical resection and adjuvant treatment. However, metastatic cancers are difficult to completely alleviate and are the cause of about $90 \%$ of cancer-related deaths (55). Stephen Paget's "seed and soil" hypothesis of metastasis claims that the distribution of cancers is not random (45). Before metastasis, the target organs are specifically modified to establish a microenvironment suitable for tumor cell growth, known as the PMN. In recent years, EVs have been shown to play a critical role in mediating the interaction between tumor cells and host cells, which prepares the pre-metastatic niche for the formation of secondary sites (56). Notably, cancer cells secrete considerably more EVs than normal cells, leading to a substantial increase in detectable EVs circulating in the blood. Exosomes have been recognized long ago, but their identification, characterization, and isolation are still under intense investigation. Moreover, whereas the definition of exosomes is based on the pathway of biogenesis, oncosomes form a functional class of extracellular vesicles (57). Indeed, oncosomes are claimed to be capable of carrying and conveying tumor-related information (58) and can mediate important cancer-related pathways to enhance tumor invasion and metastasis through different manners, including controlling angiogenesis (59), modulating stromal cells (60), 
remodeling the ECM (61), transferring malignant traits, and establishing the PMN. Exosomes containing ncRNAs and proteins play significant roles in these pathways. While the mechanism by which exosomes secreted by cancer cells act on the target tissue has been elucidated, it has also been shown that exosomes secreted by the primary lesion lead to the formation of the metastatic lesion in distant tissues (i.e., PMN formation) before the cancer cells themselves migrate to the particular tissue. Baglio et al. showed that extracellular vesicles secreted by the tumor promote osteosarcoma progression via TGF- $\beta$ signaling-induced IL- 6 production by MSCs (14). Moreover, Peinado et al. demonstrated that exosomes from highly metastatic melanomas increase the metastatic behavior of primary tumors by permanently educating bone marrow progenitors through the receptor tyrosine kinase MET (15).

However, there are many details that remain unknown, and recent research findings also raise new questions. For instance, while the content of exosomes varies among cells and changes even for the same cell depending on the environment, the mechanism for this diversity is yet to be understood. Elucidating the role of the molecules on the exosomal surface and those encapsulated within may help reveal the pathological conditions caused by the exosome and develop methods to counter them.

\section{Melanoma}

Melanoma is a highly invasive, metastatic cancer with poor prognosis and survival rate. According to the World Health Organization, 287,800 new cases of melanoma were diagnosed worldwide in 2018 , and about 60,715 cancer deaths ( $0.7 \%$ of all cancer deaths) were due to cutaneous melanoma in the same year. The incidence and mortality rates of cutaneous melanoma differ widely across the globe depending on access to early detection and primary care. In 2018, the age standardized (world standard population) incidence was 12.6 per 100,000 person-year in the EU (62).

A melanoma develops through malignant transformation of melanocytes. The pathogenic features of melanomas are characterized by their growth and - with the amplification of atypical melanocytes - self-sufficiency of growth factors, insensitivity to growth inhibitors, evasion of cellular apoptosis, limitless replicative potential, sustained angiogenesis, tissue invasion, and metastasis. These pathogenic events may result from the complex interaction between exogenous and endogenous triggers as well as tumor-intrinsic and immune-related factors (63). Similarly to other cancers, melanoma development and progression follows a sequential genetic model that results in activation of oncogenes or inactivation of tumor-suppressor genes (64). At the protein level, some genetic alteration (mutations in BRAF or TERT promoters; $C D K N 2 A, P T E N$ and TP53 genes) leads to a reciprocal overstimulation of the affected cellular 
pathways, mainly the mitogen-activated protein kinase (MAPK) pathway, the phosphoinositide 3-kinase (PI3K), protein kinase B (AKT), phophatase and tensine homologue (PTEN), and mammalian target of rapamycin (mTOR) pathway (Figure 4) (64-67).

Melanoma cells are able to escape the immune system, and modify the immune response in order to survive, for example, by reinforcing immune checkpoints. The cytotoxic T lymphocyte associated antigen-4 (CTLA-4) on T-cells competitively binds to B7 ligands on melanoma cells, interfering with CD28 interactions, thus preventing costimulation and the priming phase of T-cell activation. Additional immunosuppressive mechanisms include the downregulation of tumor-associated antigens and MHC I (68). The Janus kinase (JAK), and signal transducer and activator of transcription (STAT) pathway is a major regulator of the programmed cell death protein 1 (PD-1) immune checkpoint. Upon tumor antigen recognition by $\mathrm{T}$-cells, the interferons released trigger JAK-STAT-mediated

Figure 4. Crucial pathways in melanoma pathogenesis.

(a) MAPK, PI3K-AKT signaling, and cell-cycle regulation under normal conditions permits balanced control of basic cell functions.

(b) In melanomas, genetic alterations lead to constitutive pathway activation with loss of cellular homeostasis.

(c) The PD-1-PD-L1 immune checkpoint is primarily regulated by interferon- $\gamma$ signaling.
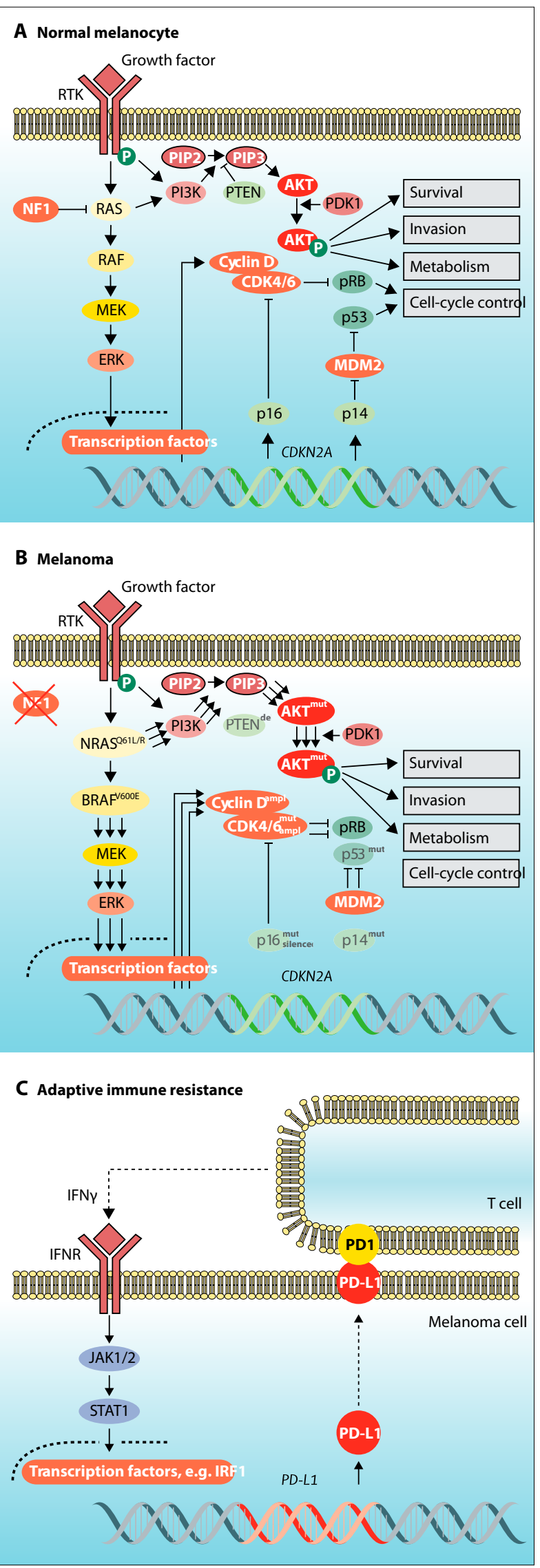
expression of PD-1 ligands (PD-L1 and PD-L2) on the surface of melanoma cells. Interaction of PD-1 with its ligands leads to the suppression of T-cell effector activity and inhibits the antitumor immune response (69).

Notably, PD-1 expression is not a default property of melanoma cells. Of further importance, Kleffel et al. recently demonstrated that PD-1 overexpressing melanoma cell subpopulations are especially aggressive, and that melanoma PD-1:PD-L1 interactions modulate mTOR signaling, whereby they interfere with programmed cell death (70). This work addressed the consequences of PD-1 overexpression and experimental inhibition of PD-1 functions (using Pdcd1 shRNA or anti-PD-1 antibodies). However, it failed to define the factors whose activation leads to the generation of PD-1 overexpressing subpopulations. This question is especially intriguing, if one considers that one of the most effective immunotherapies available today is based on the blockade of the PD-1:PD-L1 interaction mentioned above.

In addition to genetic changes in melanocytes, the interaction between the elements of the microenvironment is also a key part of melanoma formation. The melanoma microenvironment is also markedly heterogenic and involves a wide variety of collocated cells: fibroblasts, macrophages, lymphocytes, other immune cells, mesenchymal stem cells, adipocytes and cells from the structural elements of cutaneous blood vessels sunk in the extracellular matrix (71). 


\section{AIMS}

The TME includes nonmalignant cells, immune cells, secreted proteins, extracellular vesicles, blood vessels, etc. that surround and support the growth of the tumor. Interactions between the various components of the TME are crucially important; tumor cells can change the nature of the microenvironment, and conversely, the microenvironment can affect the spread and growth of tumors. The structure and composition of the TME varies among different types of cancers. In this study, we aimed to investigate how TME factors promote the pro-metastatic characteristics of melanoma. Pro-metastatic characteristics often depend on the specific tissue microenvironment. The TME was investigated from two different points of view - from the aspect of tumor, and from the behavior of tumor-infiltrating immune cells.

We aimed to investigate how tumor progression is influenced on the one hand by the tumorderived exosomes, and, on the other hand, by the various types of tumor-infiltrating macrophages (M1, M2).

We hypothesized that the tumor-derived exosomes promote tumor progression, whereas the M1 polarization of tumor-associated macrophages induces tumor regression.

In this project, we addressed four specific aims:

1. Investigate the cellular and molecular signals of melanoma-derived exosome-induced, intercellular communication-mediated malignant transformation of MSC cultures.

2. Inspect the alteration in the expression of PD-1, a melanoma progression marker and therapeutic target, upon exposure to melanoma-derived exosomes.

3. Examine the melanoma-derived exosome-induced tumor progression in vivo.

4. Evaluate the impact of macrophage polarization on tumor regression. 


\section{MATERIALS AND METHODS}

\section{Mice}

C57BL/6N and immunodeficient NOD/Scid IL2rg null (NSG) mice were obtained from Charles River Laboratories, Sulzfeld, Germany. Mice were housed in pathogen-free conditions and experiments were performed in accordance with national (1998. XXVIII; 40/2013) and European (2010/63/EU) animal ethics guidelines. The experimental protocols were approved by the Animal Experimentation and Ethics Committee of the BRC-HAS and the Hungarian National Animal Experimentation and Ethics Board (clearance numbers: XVI./03521/2011, CSI/01/3929-4/2017, XVI./03521/2011, and CSI/01/3929-4/2017).

\section{Cell cultures and cell line}

The B16F1 mouse melanoma cell line was obtained from ECACC and cultured in Dulbecco's modified Eagle's medium (DMEM, Lonza) containing 10\% fetal bovine serum (Euroclone), $0.01 \%$ sodium pyruvate, $1 \%$ MEM non-essential amino acids, $1 \%$ MEM vitamin solution, 2 mM L-glutamine and 1\% Penicillin-Streptomycin-Amphotericin B Mixture (all from Lonza).

Murine MSCs were isolated from C57BL/6N (Charles River Laboratories) 8-week-old male mice (72). Abdominal inguinal fat pads were excised, rinsed with RPMI 1640 medium, transferred to sterile tissue culture dishes and mechanically dissociated. The dissociated tissue was resuspended in RPMI 1640 containing $100 \mu \mathrm{g} / \mathrm{ml}$ collagenase (Sigma-Aldrich) and incubated at $37^{\circ} \mathrm{C}$ for $50 \mathrm{~min}$. Collagenase was neutralized with a growth medium containing $10 \%$ fetal bovine serum (FBS). After centrifugation at $470 \times \mathrm{g}$ for $15 \mathrm{~min}$, cell pellets were resuspended and washed in the culture medium. After the centrifugation, cell pellets were resuspended in complete MecenCult medium (Stemcell Technologies) and filtered through a $100 \mu \mathrm{m}$ cell strainer (BD Biosciences) to tissue culture dishes and cultured for $48 \mathrm{~h}$ at $37^{\circ} \mathrm{C}$ in $5 \% \mathrm{CO}_{2}$ and 90\% humidity. Unattached cells and debris were then removed and fresh medium was added to the adherent cells. The cells were cultured to $80 \%$ confluence before being released with trypsin-EDTA and sub-cultured. 
The purity of MSC cultures were checked by flow cytometry analysis using the Mouse Multipotent Mesenchymal Stromal Cell Marker Antibody Panel (R\&D Systems) according to the manufacturer's instructions.

\section{Cell cultures for in vitro experiments}

Passage 2 MSCs $\left(6 \times 10^{4}\right.$ cell $\left./ \mathrm{ml}\right)$ were plated in cell culture dishes $\left(1.5 \times 10^{4} / \mathrm{cm}^{2}\right)$. After $24 \mathrm{~h}$ incubation, MSC cultures were exposed to B16F1-derived exosomes (40 $\mu \mathrm{g} / \mathrm{ml}$ exosomal proteins; $1.5 \times 10^{11}$ exosomes) every 24 hours. Samples were exposed to exosomes for $24 \mathrm{~h}$, $48 \mathrm{~h}, 72 \mathrm{~h}$ and $96 \mathrm{~h}$ and then harvested in method-competent buffer.

\section{Mouse model for exosomal treatment}

B16F1 melanoma cells were administered intravenously $\left(1 \times 10^{5}\right.$ cell/100 $\left.\mu \mathrm{l}\right)$ to 6 to 8 -week-old female C57BL/6N mice. One week later, tumor bearing mice were randomized and divided into 3 groups ( $n=10$ /group). Mice were injected intravenously with control buffer $(100 \mu l)$, exosomeexposed MSCs $\left(1 \times 10^{5}\right.$ cell/100 $\left.\mu \mathrm{l}\right)$ or exosomes $(40 \mu \mathrm{g} / 100 \mu \mathrm{l}) 7,8,9,10$, and 11 days after the tumor injection (Table S1). One week after the first MSC administration, 3 animals/group were euthanized, their lungs were removed, photographed and stored at $-80^{\circ} \mathrm{C}$ for further protein, mRNA and histological analysis. The remained mice were observed for 10 more days. At the end point, the animals were euthanized, and the tumor metastases were investigated not only in their lung, but also in their entire body and removed for histological analysis. Experiments were repeated 3 times.

\section{Tumor coverage}

The tumor coverage of lungs was determined by the analysis of acquired images using the ImageJ software. The area of tumors and the healthy regions was measured, and the mean percentage, SD and $\mathrm{p}$ values were calculated in Microsoft Excel.

\section{Quantification of metastases-associated vessel diameter}

From native animal lungs, $4 \mu \mathrm{m}$ cryostat sections were made on silanized slides, then fixed and retrieved by Fix\&Perm A-B solution (Thermo Fisher Scientific) for 20 minutes. Sections were counterstained by conventional hematoxilin for 30 minutes, then washed in tap water and coverslipped. Sections were digitalized by automatic slide-scanner (3DHistech, Hu), using the software 3DHISTECH Pannoramic Viewer (3DHistech, Hu). Strict tumor border was carefully marked, then vessel diameters were measured. 


\section{The mouse model for Chlamydia treatment}

B16F1 melanoma cells (DTP, DCTP Tumor Repository) were administered intravenously $\left(1 \times 10^{5}\right.$ cell/100 $\left.\mu \mathrm{l}\right)$ to 6 to 8 -week-old female immunocompetent C57BL/6 or immunodeficient NOD/Scid IL2rg null (NSG) mice. One week after tumor cell administration, mice were treated with Chlamydia pneumoniae (C. pneumoniae) strain CWL-029 propagated in Hep2 cells (73). C. pneumoniae and the mock control (processed Hep2 cells) were heat-inactivated at $90^{\circ} \mathrm{C}$ for 30 minutes. Mice were mildly sedated with sodium pentobarbital $(7.5 \mathrm{mg} / \mathrm{ml})$ and treated intranasally with $1 \times 10^{6}$ IFU $C$. pneumoniae 7, 9, 11, 14, and 16 days after tumor implantation. Since the physical condition of NSG mice deteriorated extremely rapidly, animals were euthanized at day 14 after the third $C$. pneumoniae treatment. The special advantages of this model are: (i) with intravenous injection of melanoma cells, visible lung tumor metastases develop within 7 days after injection without significant spreading into other organs; and (ii) C. pneumoniae is a lung-specific intracellular pathogen with a significant invasion rate even to the lung metastases. Two hours after the first inhalation (day 7), 4 hours after 2nd, 12 hours after 3rd and 24 hours after 5th inhalation, 3 animals/group were anaesthetized and their lungs were harvested for protein, mRNA and histological analysis. The remaining mice received the 4th (day 14) and the 5th (day 16) treatments and were followed for survival. At the endpoint, the animals were euthanized, their lungs were removed and 3 independent persons counted the number of surface metastases in a blind fashion.

For the survival experiments, groups of mice $(n=15)$ were treated as described 5 times after melanoma implantation. Kaplan-Meier survival curves were analyzed by a log-rank statistical test and $p \leq 0.05$ was regarded as statistically significant. All animal experiments were authorized by the institutional and national animal welfare committees.

\section{Exosome isolation and characterization}

Exosomes were isolated by adapting the protocol of Peinado et al. (15) from melanoma cell culture supernatant. B16F1 supernatants were harvested, supplemented by complete protease inhibitor cocktail (Roche) and centrifuged at $780 \times \mathrm{g}$ for $5 \mathrm{~min}$ at $4^{\circ} \mathrm{C}$ to remove intact cells. Then, the supernatants were centrifuged at $3,900 \times \mathrm{g}$ for $15 \mathrm{~min}$ at $4^{\circ} \mathrm{C}$ and filtered through a $0.2 \mu \mathrm{m}$ membrane (Millipore) to remove larger cell fragments and microvesicles. Exosomes were pelleted by ultracentrifugation at $150,000 \times \mathrm{g}(\mathrm{T}-1270$ rotor at $40,500 \mathrm{rpm})$ for $1 \mathrm{~h}$ at $4^{\circ} \mathrm{C}$. The pellet was washed twice and resuspended in DPBS and stored at $-80^{\circ} \mathrm{C}$.

The concentration of exosomal proteins was determined using a Pierce BCA Protein assay kit (Thermo Fisher Scientific) and a Benchmark Microplate Reader (Bio-Rad,) according to the manufacturer's instructions. 


\section{Determination of shape and size of the isolated vesicles}

\section{Scanning Electron Microscopy (SEM)}

Five microliters of isolated exosomes in DPBS were spotted on glass coverslips coated with $0.01 \%$ Poly-L-Lysine (Sigma-Aldrich) and incubated overnight at $4{ }^{\circ} \mathrm{C}$. Using sterile forceps, coverslips were transferred into a plastic plate. Exosomes were fixed by gently adding $2 \%$ paraformaldehyde buffer $\mathrm{pH} 7.2$ diluted in DPBS for $30 \mathrm{~min}$. The coverslips were washed twice with DPBS and dehydrated with a graded series of ethanol $(40 \%, 60 \%, 80 \%$, and $100 \%$ ethanol, each for $5 \mathrm{~min})$. The samples were dried with a critical point dryer (QUORUM K850) and the coverslips were mounted onto a microscope stub one at a time using carbon tape, followed by a $7 \mathrm{~nm}$ gold coating (QUORUM Q150) and observed under a field-emission scanning electron microscope (JEOL JSM-7100F/LV).

\section{Atomic force microscopy (AFM)}

Exosomes were allowed to adsorb to the freshly cleaved muscovite mica (SPI-ChemTM Mica Sheets, Structure Probe, Inc.) surface directly from buffer solution (DPBS, Lonza). All AFM measurements were carried out with an Asylum MFP-3D head and Molecular Force Probe Controller (Asylum Research), using the Asylum Research MFP-3D program (version 15.09.112) written in Igor Pro software (version 6.37, Wavemetrics). Image processing and data calculation were carried out using the same software. Rectangular silicon cantilevers (OMLC-AC240TS, Olympus Optical Co., Ltd.) with a typical spring constant of $2 \mathrm{~N} / \mathrm{m}$ were used in a dry condition and silicon nitride rectangular cantilevers with "V" shaped tips (Bio-Lever BL-RC150VB, Olympus Optical Co. Ltd.) with a typical spring constant of $0.03 \mathrm{~N} / \mathrm{m}$ in buffer solution. Typically, $512 \times 512$ points were taken at a scan rate of 1 line/s. The measurements presented here are amplitude images and height profiles.

\section{Identification of exosomal proteins by mass spectrometry}

Exosomal proteins $(24 \mu \mathrm{g})$ were separated in 4-12\% Bis-Tris Protein Gels and stained with Coomassie blue (0.1\% Coomassie Brilliant Blue R-250, 50\% methanol and $10 \%$ acetic acid).

The lanes were each cut into 12 equal bands and subjected to in-gel digestion. The gel bands were diced into smaller pieces, and the SDS and CBB dyes were washed out with $3 \times 50 \mu 1$ $25 \mathrm{mM}$ ammonium-bicarbonate $(\mathrm{ABC}) / 50 \%$ Acetonitrile $(\mathrm{AcN})$. After reduction with DTT (1,4dithiothreitol, Sigma-Aldrich; $20 \mu 1,10 \mathrm{mM}$ DTT in $25 \mathrm{mM} \mathrm{ABC}$ ) at $56^{\circ} \mathrm{C}$ for $30 \mathrm{~min}$, and alkylation with IAM (iodoacetamide, Sigma-Aldrich; $20 \mu 1,55 \mathrm{mM}$ IAM in $25 \mathrm{mM} \mathrm{ABC}$ ) at $\mathrm{RT}$ in the dark for $30 \mathrm{~min}$, the gel samples were dried in a vacuum centrifuge, and after that 
rehydrated in $20 \mu 1$ of trypsin (Sequencing Grade Modified Trypsin, Promega; $5 \mathrm{ng} / \mu 1$ in $25 \mathrm{mM}$ $\mathrm{ABC}$ ) and incubated at $37^{\circ} \mathrm{C}$. The digestion was stopped after 4 hours by lowering the $\mathrm{pH}$ of the buffer below 3 and by adding $2 \mu 1$ of $10 \%$ formic acid. Tryptic peptides were extracted from the gel with $3 \times 50 \mu \mathrm{l}$ of $2 \%$ formic acid in $50 \% \mathrm{AcN}$ and dried. Prior to mass spectrometric analysis, each sample was dissolved in $50 \mu 1$ of $0.1 \%$ formic acid.

Samples were analyzed in an LTQ-Orbitrap Elite (Thermo Fisher Scientific) mass spectrometer on-line coupled with a nanoHPLC (nanoAcquity, Waters) system. Five $\mu 1$ of each of the ingel digests were loaded (for $3 \mathrm{~min}$ at a flow rate of $8 \mu \mathrm{l} / \mathrm{min}$, using $0.1 \%$ Formic acid [FA] in 3\% Acetonitrile 97\% water) onto a reversed phase trap column (Waters, Symmetry C18, $0.180 \times 20 \mathrm{~mm}$ ) and separated on a $\mathrm{C} 18$ reversed phase (Waters, BEH300C18 $1.7 \mu \mathrm{m}$ ) nanocolumn $(0.075 \times 200 \mathrm{~mm})$. The flow rate was $330 \mathrm{nl} / \mathrm{min}$ and a linear gradient was used from $3 \%$ to $40 \%$ $\mathrm{B}$ in $37 \mathrm{~min}$ (solvent $\mathrm{A}$ was $0.1 \% \mathrm{FA}$ in water and solvent $\mathrm{B}$ was $0.1 \% \mathrm{FA}$ in Acetonitrile).

The high voltage $(1.2 \mathrm{kV})$ was applied through liquid junction between the chromatographic column and the non-coated silica nanospray emitter (NewObjective, $10 \mu \mathrm{m}$ tip ID). The mass spectrometer operated in data-dependent mode: the survey mass spectra were detected in the orbitrap with high resolution $(\mathrm{R}=60 \mathrm{k} @ \mathrm{~m} / \mathrm{z}$ : 400, mass range m/z: 380-1,400) and the most abundant multiply charged 20 peaks were selected for ion-trap fragmentation (NCE: 35\%; activation q: 0.25 ; activation time: $10 \mathrm{~ms}$; minimum signal intensity: 5,000 counts). The MS/MS spectra were detected in the ion trap. Dynamic exclusion was used; the precursors were excluded for $15 \mathrm{sec}$ after the first fragmentation event.

Data analysis: searchable peaklists (mgf format) were extracted using ProteomeDiscoverer(ver:1.4 Thermo Fisher Scientific) and subjected to database search on our in-house ProteinProspector (ver: 5.14.1) search engine using the following parameters: parent ion tolerance: $5 \mathrm{ppm}$; fragment ion tolerance: $0.6 \mathrm{Da}$; Cys carbamidomethylation was set as constant, and Met oxidation, cyclisation of peptide $\mathrm{N}$-terminal Glu to pyroglutamic acid, protein $\mathrm{N}$ terminal acetylation were set as variable modifications. Only fully tryptic peptides were considered with a maximum of 2 missed cleavage sites. The Mus musculus and Bos taurus protein sequences of the Uniprot (UniProtKB.06.11.2014) database completed with human keratins and pig trypsin, altogether 106,330 protein sequences were searched. For the false discovery rate (FDR) estimation, the searches were performed on the database concatenated with the randomized sequences. Protein identification was accepted if the ProteinProspector expectation value was less than 0.01 , and the protein was identified with at least 2 unique peptides (expectation value less than 0.05 and score higher than 15$)$. FDR values were less than $1 \%$ in all cases.

For the functional validation, the obtained protein list was analyzed by the "Core Analysis" function included in Ingenuity Pathway Analysis (IPA, Qiagen Bioinformatics) software. 


\section{Isolation and detection of exosomal miRNAs}

miRNA sequencing was performed using SOLiD Total RNA-Seq lit for Small RNA Libraries (Applied Biosystems now part of Thermo Fisher Scientific) according to the manufacturer's instructions. Purification was performed on 10\% TBE-Urea gels stained with Sybr Gold nucleic acid gels stain (both from Invitrogen now part of Thermo Fisher Scientific). Final purification was performed using PureLink PCR Micro Kit (Invitrogen). Final libraries were quality checked using High Sense DNA kit on Bioanalyzer (all from Agilent). Concentration of each library was determined using the SOLID Library TaqMan Quantitation Kit (Life Technologies now part of Thermo Fisher Scientific). Each library was clonally amplified on SOLiD P1 DNA Beads by emulsion PCR (ePCR). Emulsions were broken with butanol, and ePCR beads enriched for template-positive beads by hybridization with magnetic enrichment beads. Template-enriched beads were extended at the 3' end in the presence of terminal transferase and 3' bead linker. Beads with the clonally amplified DNA were deposited onto a SOLiD sequencing slide and sequenced on the SOLiD 5500 Instrument using the 50-base sequencing chemistry.

Bioinformatic Analysis Raw data quality assessment, read trimming, read mapping and miRNA expression profiling was carried out in CLC Genomics Workbench tool version 8.0.2 (CLC Bio now part of Qiagen) using annotated Mus musculus miRNA sequences according to the miRBase release 21 as a mapping reference.

\section{Detection of proteins by Western blotting}

Protein samples were resuspended in 4x sample buffer (NuPAGE® LDS Sample Buffer (4X), NuPAGE® Sample Reducing Agent (10X), Thermo Fisher Scientific), boiled at $96^{\circ} \mathrm{C}$ for $10 \mathrm{~min}$, and immediately cooled on ice. Electrophoresis of the proteins was performed using $4-12 \%$ BisTris Protein Gels (NuPAGE ${ }^{\mathrm{TM}}$ Novex $^{\mathrm{TM}}$, Thermo Fisher Scientific), ProSieve ${ }^{\mathrm{TM}}$ Color Protein Markers (Lonza), and electrophoresis buffer (NuPAGETM MOPS SDS Running Buffer, Thermo Fisher Scientific) at $200 \mathrm{~V}$ and $0.03 \mathrm{~A}$ for $40 \mathrm{~min}$.

The proteins of electrophoresed gels were transferred to Immobilon transfer membrane (Millipore) using transfer buffer (NuPAGE® Transfer Buffer (20X)) at $30 \mathrm{~V}$ and 170-110 mA for $60 \mathrm{~min}$. Membranes were blocked in TBST buffer $(25 \mathrm{mM}$ Tris-HCl, $150 \mathrm{mM} \mathrm{NaCl}$, $0.05 \%$ Tween-20, $\mathrm{pH} 7.2$ ) containing $5 \%$ non-fat milk for $60 \mathrm{~min}$ at room temperature. After blocking, membranes were incubated with each primary antibody in TBST buffer containing $1 \%$ non-fat milk overnight at $4^{\circ} \mathrm{C}$. Membranes were washed three times for 10 min with TBST buffer and incubated for $60 \mathrm{~min}$ at room temperature with secondary antibody in TBST buffer containing 1\% non-fat milk. Membranes were washed three times for 10 min with TBST buffer. Bound antibodies were visualized by chemiluminescence using an ECL Plus Western 
Blotting detection system (Advansta). Immunoreactive signals were detected by using LI-COR ODYSSEY ${ }^{\circledR}$ Fc (Dual-mode imaging system) imager followed by analysis with Odyssey v1.2.

\section{Western blot analysis of exosome markers}

For exosomal marker identification, Western blot analyses were performed with an anti-CD63 polyclonal antibody (1:250 dilution; Biorbyt), an anti-CD9 rabbit monoclonal antibody (clone: EPR2949, 1:500 dilution, LifeSpan Biosciences), an anti-CD81 hamster monoclonal antibody (clone: EAT2, 1:1,000 dilution, LifeSpan Biosciences), an anti-HSP70 monoclonal antibody (clone: C92F3A-5, 1:8,000 dilution; Enzo Life Sciences), an anti-rabbit IgG HRP-Conjugated antibody (1:1,000 dilution, R\&D Systems), and an anti-Hamster IgG HRP-Conjugated antibody (1:30,000 dilution, Thermo Fisher Scientific).

\section{PD-1 detection by Western blotting}

The cells were washed three times in PBS, then lysed in TENT Buffer $(50 \mathrm{mM}$ Tris-HCl, $2 \mathrm{mM}$ EDTA, $150 \mathrm{mM} \mathrm{NaCl}$, 1\% TritonX-100, completed with 1x protease inhibitor cocktail (Roche). Protein samples were separated and blotted as described above.

For PD-1 protein level detection, an anti-PD-1 mouse monoclonal antibody (clone: RMP1-14, 1:1,000 dilution, Biolegend) and an anti-rat IgG HRP-conjugated secondary antibody (1:500 dilution, R\&D Systems) was used.

\section{Cytokine and chemokine detection by proteome profiling}

Lung samples were lysed in NP40 cell lysis buffer (Thermo Fisher Scientific), and protein content was measured by the Pierce BCA Protein Assay kit (Thermo Fisher Scientific). Expression levels of different cytokines in pooled lung specimens were determined using Mouse Cytokine Array Panel A (R\&D Systems), according to the manufacturer's instructions. Immunoreactive signals were detected by using LI-COR ODYSSEY ${ }^{\circledR}$ Fc (Dual-mode imaging system) imager followed by analysis with Image Studio Lite v5.2.

\section{Quantitative reverse transcription PCR (qRT-PCR)}

\section{Mlana and Mitf qRT-PCR}

Total RNA of biological samples was purified using the Quick-RNA MiniPrep isolation kit of \#R1054S (Zymo Research). All the preparation steps were carried out according the 
manufacturer's instructions. RNA samples were stored at $-80^{\circ} \mathrm{C}$ in the presence $30 \mathrm{U}$ of Prime RNAse inhibitor (Fermentas, part of Thermo Fisher Scientific) for further analysis. The quantity of isolated RNA samples was checked by spectrophotometry (NanoDrop 3.1.0).

qRT-PCR was performed on a RotorGene 3000 instrument (Corbett Research) with genespecific primers and SybrGreen protocol to monitor gene expression. $1 \mu \mathrm{g}$ of total RNA was reverse transcribed with random primers using the High-Capacity cDNA Archive Kit (Applied Biosystems) according to the manufacturer's instructions in final volume of $30 \mu \mathrm{l}$. The temperature profile of the reverse transcription was the following: $10 \mathrm{~min}$ at room temperature, 2 hours at $37^{\circ} \mathrm{C}, 5 \mathrm{~min}$ on ice and finally $10 \mathrm{~min}$ at $75^{\circ} \mathrm{C}$ for enzyme inactivation. These steps were carried out in a Thermal Cycler machine (MJ Research, Marshall Scientific). After dilution with $30 \mu \mathrm{l}$ of water, $1 \mu \mathrm{l}$ of the diluted reaction mix was used as template in the qRT-PCR. Reactions were done with FastStart SYBR Green Master mix (Roche) according to the manufacturer's instructions at a final primer concentration of $250 \mathrm{nM}$ under the following conditions: $15 \mathrm{~min}$ at $95^{\circ} \mathrm{C}, 40$ cycles of $95^{\circ} \mathrm{C}$ for $15 \mathrm{sec}, 60^{\circ} \mathrm{C}$ for $25 \mathrm{sec}$ and $72^{\circ} \mathrm{C}$ for $25 \mathrm{sec}$. The fluorescence intensity of SybrGreen dye was detected after each amplification step. Melting temperature analysis was done after each reaction to check the quality of the products. Primers were designed using the online Roche Universal Probe Library Assay Design Center. The quality of the primers was verified by MS analysis provided by Bioneer (Daejeon, Korea). Relative expression ratios were calculated as normalized ratios to MmRpl27 (Mus musculus ribosomal protein L27) gene. Nontemplate control sample was used for each PCR run to check the primer-dimer formation. The final relative gene expression ratios were calculated as delta-delta $\mathrm{Ct}$ values. Information about the genes and the primers is collected in Table S2.

\section{TaqMan panel of 40 oncogenes}

qRT-PCR was performed on an ABI Prism 7000 sequence detection system (Applied Biosystems) using specific pre-designed customized 96-well TaqMan Array oncogene panels (Thermo Fisher Scientific) containing 44 primers and probes specifically selected based on extensive literature work. RNA was isolated from MSC cells with Qiagen RNeasy mini kit (Qiagen) based on the manufacturer's instructions. RNA concentrations were measured by NanoDrop spectrophotometer (NanoDrop). cDNAs were reverse transcribed with TaqMan Reverse Transcription Reagents (Thermo Fisher Scientific) following the manufacturer's instructions. For the TaqMan qRT-PCR panel, cDNA mixture of three parallel samples was used for each of the following conditions: control, $6 \mathrm{~h}, 24 \mathrm{~h}$ and $72 \mathrm{~h}$ exosome treatment applied on MSC cells derived from four mice. $80 \mathrm{ng}$ cDNA and TaqMan Gene Expression Master Mix (Thermo Fisher Scientific) was used for the qRT-PCR experiment. 
Total RNA was isolated using TRIzol (Thermo Fisher Scientific) from the lung samples, DNase treatment was performed according to the manufacturers' protocol, and then total RNA was transcribed into cDNA using the High Capacity cDNA Kit (Thermo Fisher Scientific). PCR amplification was performed using TaqMan primers and probes, and thermal cycle conditions were set as follows: $2 \mathrm{~min}$ at $50^{\circ} \mathrm{C}, 10 \mathrm{~min}$ at $95^{\circ} \mathrm{C}, 40$ cycles of $15 \mathrm{sec}$ at $95^{\circ} \mathrm{C}$ and $1 \mathrm{~min}$ at $60^{\circ} \mathrm{C}$. As internal control transcripts of ACTB (Assay ID: Mm00607939_s1), GAPDH (Assay ID: Mm99999915_g1) and PPIA (Assay ID: Mm02342430_g1) were determined. The amount of the transcripts was normalized to those of the housekeeping genes using the $\Delta \mathrm{Ct}$ method. Finally, the results were normalized to the expression of the vehicle control ( $\Delta \Delta \mathrm{Ct}$ method).

For relationship discovery, Hierarchical Cluster Analysis was performed by R software. In detail, the "bottom up" agglomerative hierarchical clustering strategy was used, and the results were presented in a tree-based dendrogram. (R software: R. D. C. Team, R: A Language and Environment for Statistical Computing, R Foundation for Statistical Computing, Vienna, Austria, 2008).

\section{Cytokines and chemokines qRT-PCR}

Total RNA of lungs was purified using a NucleoSpin RNA II RNA isolation kit; first-strand cDNA was synthesized and qRT-PCR reactions were performed on pooled samples $(n=3)$ on a RotorGene 3000 instrument with gene-specific primers (CCL2, CCL3, CD86, IL-12, IL-6, IL-10, CXCL16, CCL7, CD80, CXCL11, CXCL9, IL-23, TNF- $\alpha$ ), and SYBR Green protocol to monitor gene expression. Each individual $\mathrm{Ct}$ value was normalized to the average $\mathrm{Ct}$ values of four internal control genes ( $\Delta \mathrm{Ct}$ values). The final relative gene expression ratios (fold change) were calculated as comparisons of $\Delta \mathrm{Ct}$ values ( $\Delta \Delta \mathrm{Ct}$ values). Non-template control sample was used for each PCR run to check the primer-dimer formation. Primer sequences are available upon request.

\section{Histology, immunohistochemistry}

Lung specimens were fixed in $4 \%$ buffered formaldehyde; then, routine HE histology as well as standardized immunohistochemistry tissue microarray were performed using anti-CD11b (clone M1/70; R\&D Systems) and anti-CD80 (B7-1; R\&D Systems) antibodies.

\section{Visualization of labeled exosome internalization in MSCs}

To examine the uptake of exosomes by MSCs, cells were plated to black 24-well Visiplates $\left(1 \times 10^{4}\right.$ cells/well $)$ and incubated for $24 \mathrm{~h}$. The exosomes were labeled with Dil dye 
(1,1'dioctadecyl-3,3,3',3'-tetramethylindocarbocyanine perchlorate, PromoKine) and the MSC cultures were labeled with $\mathrm{DiO}$ dye (3,3'-dioctadecyloxacarbo-cyanine perchlorate, PromoKine) according to the manufacturer's instructions. Dil-labeled exosomes were washed in PBS by ultracentrifugation (at $150,000 \times \mathrm{g}$ for $1 \mathrm{~h}$ at $4^{\circ} \mathrm{C}$ ). Forty micrograms per milliliter Dil-labeled exosomes were added to DiO-labeled MSC cultures, and the exosome uptake was followed for $24 \mathrm{~h}$ in the Celldiscoverer 7 automated live cell imaging system (Zeiss). After $24 \mathrm{~h}$, the cells were fixed with $4 \%$ paraformaldehyde solution and a nucleus staining was performed using DAPI (Life Technologies now part of Thermo Fisher Scientific). Then, 5 image z-stacks were acquired for both channels by Operetta High Content Screening System (PerkinElmer). The stacks were maximum intensity projected and then analyzed automatically using a customized version of CellProfiler (74). Nuclei were detected with Otsu-adaptive threshold combined with diameter-based filtering, then cytoplasms were identified with propagation method seeded from the nuclei and using the exosome channel. Exosomes were located with a customized version of A-trous wavelet transform based spot detection (75). Several wavelet levels were used to ensure the detection of exosomes with various sizes and then the overlaps were removed based on circularity measures. Finally, the exosome numbers per cell were identified using MATLAB programming, the diagrams were created in Microsoft Excel.

\section{Cell proliferation}

After $72 \mathrm{~h}$ incubation, exosome-exposed and control MSC cultures were dissociated with trypsin from the culture surface. Cells were washed in medium and counted in a Bürker chamber and a cell counter (Bio-Rad, TC10 Automated Cell Counter).

\section{Detection of apoptosis}

Exosome-exposed MSCs and control cells were treated with $100 \mathrm{ng} / \mathrm{ml}$ mouse TNF- $\alpha$ (R\&D Systems). After $24 \mathrm{~h}$ incubation, cell death was determined by the Annexin V Apoptosis Detection Kit with PI (Biolegend) according to the manufacturer's recommendations. Samples were measured by FACS Calibur flow cytometer (BD Biosciences), data were analyzed by Flowing Software (Cell Imaging Core, Turku Centre for Biotechnology) where percent of positive cells was determined by relative fluorescence intensity, and the results were expressed as mean of percentage of positive cells $(\%) \pm \mathrm{SD}$. Cells that are annexin V/propidium iodide (PI) double positive show the sign of late apoptosis, while cells that are annexin V positive and PI negative indicate early apoptosis. Annexin V negative and PI positive cells are necrotic; viable cells are both annexin V and PI negative. 


\section{PD-1 and MLANA detection by immunofluorescence microscopy}

MSC cultures were fixed with $4 \%$ paraformaldehyde for 10 min, permeabilized with $0.1 \%$ Triton X-100, and blocked in PBS buffer containing 5\% BSA (Sigma-Aldrich) for $60 \mathrm{~min}$ at room temperature. After blocking, cells were incubated with primary antibody in PBS buffer containing 1.2\% BSA overnight at $4^{\circ} \mathrm{C}$. As primary antibodies, 1:200 anti-PD-1 (clone: RMO114, Biolegend) or 1:200 anti-MLANA (Byorbit) were used. Then, cells were incubated for $1 \mathrm{~h}$ at room temperature with 1:100 Alexa Fluor 647 conjugated anti-rat antibody (Jackson ImmunoResearch Laboratories) or 1:500 Alexa Fluor 555 conjugated anti-rabbit antibody (Thermo Fisher Scientific). Tubulin was stained with 1:500 Alexa Fluor 488 conjugated antitubulin- $\alpha$ antibody (clone: 10D8, Biolegend). Nucleus staining was performed using DAPI (Life Technologies). Slides were washed three times with DPBS between each step. Images were obtained at 60x magnification using an Olympus confocal laser scanning microscope.

\section{PD-1 detection by STORM super-resolution imaging}

All dSTORM super-resolution experiments were performed on a custom-made inverted microscope based on a Nikon Eclipse Ti-E frame. After conditioning (through spatial filtering via fiber coupling and beam expansion), the applied laser beams were focused onto the back focal plane of the microscope objective (Nikon CFI Apo 100x, NA = 1.49), which produced a collimated beam on the sample. The angle of illumination was set via a tilting mirror mounted into a motorized gimbal holder and placed onto the conjugate plane of the sample. All the dSTORM images were captured under EPI illumination at an excitation wavelength of $647 \mathrm{~nm}$ (Nikon: $647 \mathrm{~nm}, 300 \mathrm{~mW}$ ). The laser power, controlled via an acousto-optic tunable filter (AOTF), was set to $4 \mathrm{~kW} / \mathrm{cm}^{2}$ on the sample plane. An additional laser (Nichia, $405 \mathrm{~nm}, 60 \mathrm{~mW}$ ) was used for both reactivation and reference measurements. Images were captured by an Andor iXon3 897 BV EMCCD digital camera $(512 \times 512$ pixels with a pixel size of $16 \mu \mathrm{m})$. The size of the illuminated region of the sample was matched to the size of the detector, which determined the field of view $\left(\mathrm{FOV}=80 \times 80 \mu \mathrm{m}^{2}\right)$. Frame stacks for dSTORM super-resolution imaging were typically captured at a reduced image size (crop mode), when only the central $128 \times 128$ pixel region was selected. A fluorescence filter set (Semrock, LF405/488/561/635-A-000) was used to select and separate the excitation and emission lights in the microscope. Additional emission filters (Semrock, BLP01647R-25) were used in the detection path to further clean the fluorescent light spectrally for the reduction of spectral crosstalk.

During the measurements, the perfect focus system of the microscope was used to keep the sample in focus with a precision of $<30 \mathrm{~nm}$. The storage buffer on the sample was replaced with a special switching buffer (76). Typically, 10,000 frames were captured with an exposure time 
of $30 \mathrm{~ms}$. Reference images with full size FOV were captured at low intensity when the majority of fluorescent molecules were still active and the overall structure of the labeled sample could be visualized.

The captured and stored image stacks were evaluated and analyzed by rainSTORM localization software (77). The individual images of single molecules were fitted with a Gaussian point spread function and their center positions were associated with the position of the fluorescent molecule. Localizations were filtered via their intensity, ellipticity and standard deviation values. Localizations with precisions of $<45 \mathrm{~nm}$ were only used to form the final image. The estimated mean precision of the accepted localizations was $19 \mathrm{~nm}$. Mechanical drift introduced by either the mechanical movement of the sample or thermal effects was analyzed and reduced by means of a blind drift correction algorithm. Spatial coordinates of the localized molecules were stored and the final super-resolved image was visualized. The multicolor merged images were generated by ImageJ software.

\section{Network representation by Ingenuity Pathway Analysis}

According to literature data, we established a protein network from the in vivo overexpressed genes in the exosome-related animal groups. A custom graphical representation of this network was generated using the Path Explorer tool of the Ingenuity Pathway Analysis (IPA) Path Designer. Genes are represented as red nodes, using various shapes that represent the functional class of the gene product. In a few cases, proteins are substituted with the complex, which they are a part of. To identify potential exosomal factors, which can induce the activation of the network, a list was generated from the exosomal proteins detected by LC-MS/MS and the exosomal miRNAs identified by SOLiD sequencing. The Grow tool in the IPA Path Designer revealed significant interactions between the network and the generated list (the interacting exosomal factors are listed in grey boxes and the types of relationships are indicated in parentheses). During construction in the IPA, the significance level was set to "experimentally observed" data to avoid the representation of predicted, unproven interactions.

\section{Statistical analysis}

All of the data are presented as the mean \pm SD or SEM and represent a minimum of three independent experiments. Statistical parameters including statistical analysis, statistical significance, and $\mathrm{n}$ values are reported in the Figure legends. For in vivo experiments $\mathrm{n}=$ number of animals. For statistical comparison, we performed two-tailed Student's t-test. A value of $\mathrm{p} \leq 0.05$ was considered significant (represented as ${ }^{*} \mathrm{p} \leq 0.05$, not significant [n.s.]). 


\section{RESULTS}

\section{Isolated vesicles show exosomal properties}

First, we isolated extracellular vesicles from B16F1 mouse melanoma cells. As it was shown by SEM and AFM (Figures 5a,b), the isolated fraction indeed contained exosomes, as the particles were cap-shaped, and their size was within the 40-120 nm range. Then, the presence of molecules (CD9, CD63, CD81 and HSP70) characteristic for exosomes $(51,52)$ was assessed by Western blotting (Figure 5c). Overall, the physical properties, the shape and size, and also the protein composition of the isolated vesicles meet the MISEV2018 requirements (52).

a

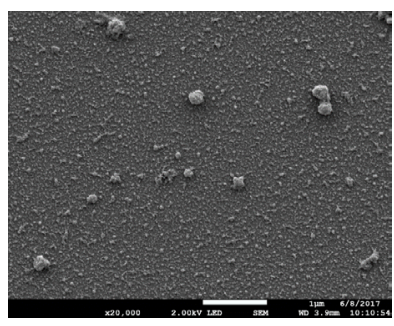

C

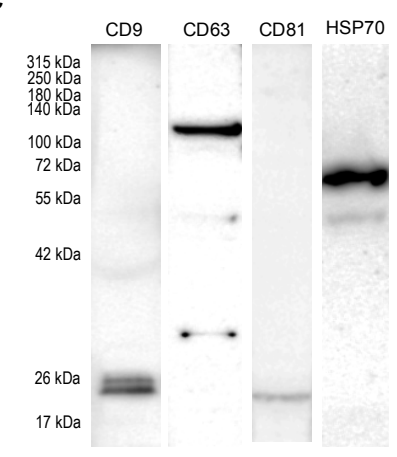

b
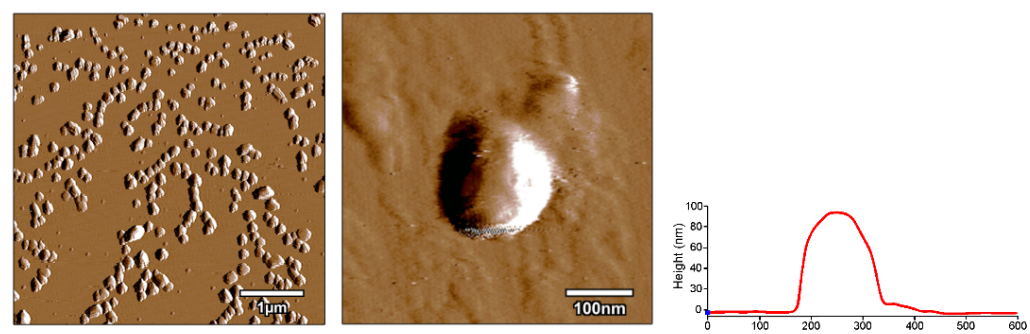

d

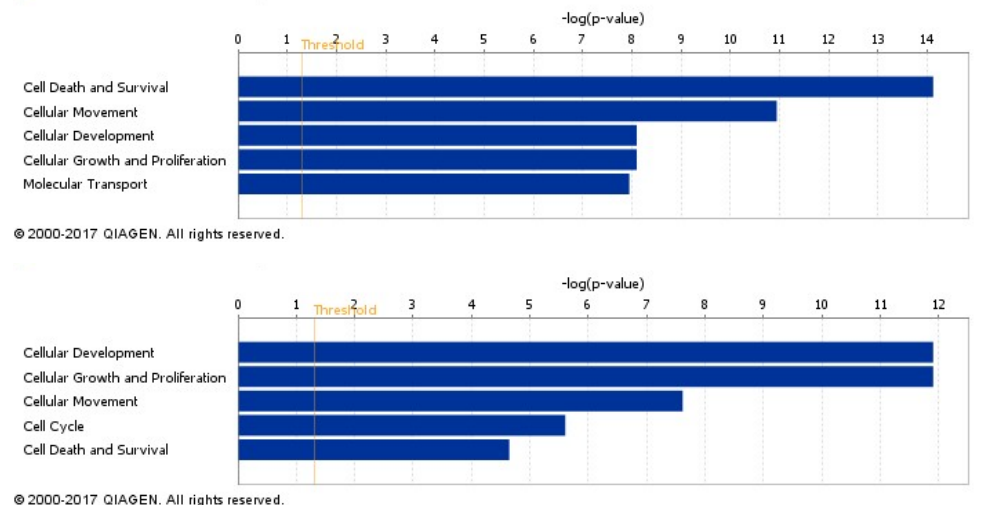

Figure 5. Characterization of B16F1 melanoma cell culture-derived exosomes.

(a) Scanning electron micrograph of exosomes secreted by melanoma cells. (b) Atomic force microscopy images of exosomes. The left and middle pictures represent the shape, and surface topography of vesicles; the graph to the right represents height profile. (c) Western blot analysis of common exosome markers (CD9, CD63, CD81 and HSP70). (d and e) The top 5 molecular and cellular functions identified by IPA of exosomal proteins and miRNAs. 
Exosomes were then subjected to large-scale analysis to determine their protein and miRNA profiles. Whole proteome analysis (using LC-MS/MS) and bioinformatics tools (used to identify elements of the exosomes' proteomics spectrum in the UniProt database, and to compare the findings with entries of the ExoCarta database) revealed that 95 distinct proteins were identified in melanoma exosomes (Table S3). These proteins exhibited an $86.3 \%$ overlap with molecules listed as characteristic exosome protein markers in ExoCarta. Furthermore, miRNA sequencing (using SOLiD 5500xl technology) identified 168 known miRNA elements (Table S4) which, similarly to the proteomics data, exhibited a large (93.5\%) overlap with molecules listed as characteristic exosome miRNA markers in ExoCarta.

To uncover the functional significance of the proteomics and miRNA sequencing data, IPA was employed. This analysis has shown that the identified proteins most probably participate in cellular and molecular processes such as "Cell Death and Survival", "Cellular Movement", "Cellto-Cell Signaling and Interaction", "Cellular Growth and Proliferation" and "Cell Morphology" $\left(\mathrm{p}_{\text {range }}=7.53 \times 10^{-15}-9.32 \times 10^{-4}\right)$ (Figure $5 \mathrm{~d}$ ). Very similarly to these data, functions of the identified miRNAs were suggested to be linked to mechanisms of "Cellular Development", "Cellular Growth and Proliferation", "Cellular Movement", "Cell Cycle" and "Cell Death and Proliferation" $\left(\mathrm{p}_{\text {range }}=1.25 \times 10^{-12}-4.88 \times 10^{-2}\right)$ (Figure 5e).

\section{Tumor exosome exposure resulted in oncogenic reprogramming of MSCs in vitro}

Then, we investigated the impact of exosomes on the biological processes (e.g. proliferation, survival, malignant transformation, etc.) of MSCs, which are generally considered as adequate in vitro models of tumor stroma (14). For these experiments, MSC cultures were initiated from mouse abdominal adipose tissue (72) and were subjected to melanoma exosome treatment.

First, we assessed whether exosomes were internalized by MSCs. High-throughput microscopy showed that MSCs (labeled green by the $\mathrm{DiOC}_{18}$ (3) lipid dye) indeed took up exosomes (labeled red by the $\mathrm{DilC}_{18}$ (3) lipid dye) as early as $1-2 \mathrm{~h}$ after application (Figure S1). Importantly, after $24 \mathrm{~h}$, the majority of MSCs were populated by exosomes (Figure 6a,b). Indeed, image analysis and statistical evaluation revealed a $91 \%$ internalization efficacy. This suggests that functional alterations demonstrated by this study were due to exosome-induced cell-population, and not individual cell level effects.

Then, we determined whether the internalized exosomes could induce a melanoma-like malignant transformation of the MSCs. By employing two complementary cell-counting methods, we found that proliferation rate of the MSCs significantly accelerated $72 \mathrm{~h}$ after exosome exposure (Figure 6c). Flow cytometry also showed that the exosome-treated MSCs exhibited a partial 
resistance to the cell death-inducing effects of $100 \mathrm{ng} / \mathrm{ml} \mathrm{TNF}-\alpha$, as the fraction of the dead cells was significantly decreased in these cultures (Figure 6d).

Since the exosomes were isolated from melanoma cells, we were then intrigued to see whether the above alterations (which all argue for the malignant transformation of the susceptible cells) also resulted in de novo appearance of melanoma-specific features in the transformed MSCs. To answer this question, we assessed the expression of the melanoma-specific markers MLANA and MITF. By qRT-PCR, we found that mRNA transcript levels of both markers elevated markedly in MSCs upon exosome treatment (Figure 6e and Figure S2), albeit the kinetics of elevation of the two molecules were slightly different. Moreover, in good accordance with the mRNA data, immunofluorescence labeling showed that exosome exposure markedly increased the expression of MLANA at the protein level as well (Figure 6f). 


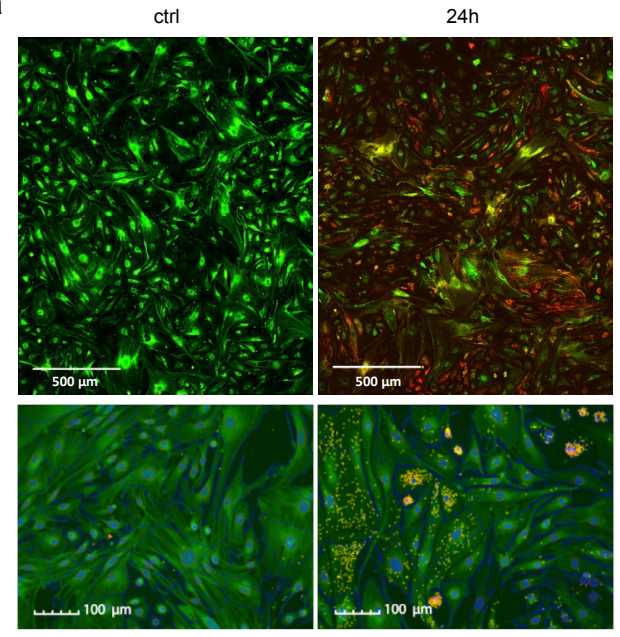

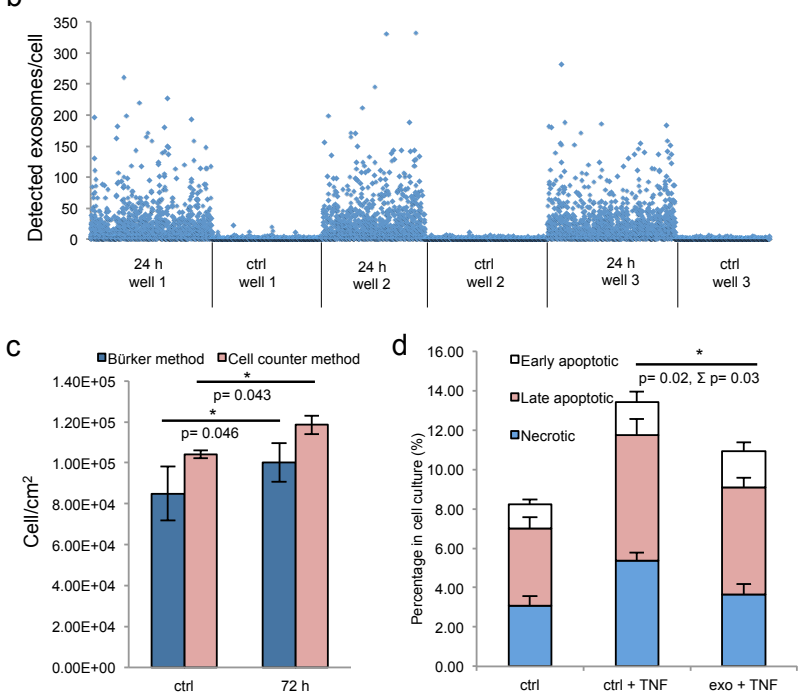

e

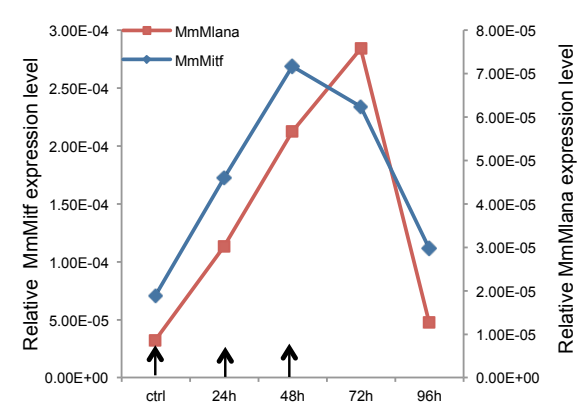

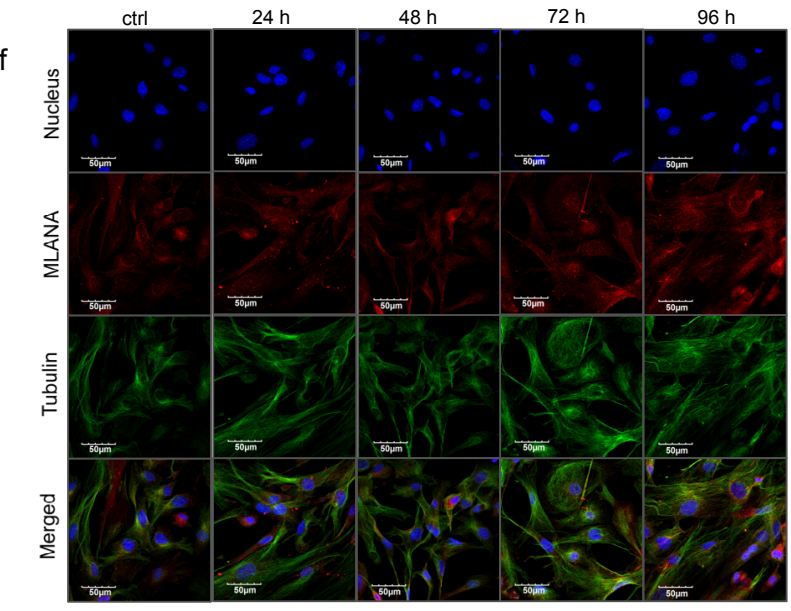

Figure 6. Internalized melanoma exosomes induce malignant transformation of the recipient MSCs.

(a) Fluorescent images of exosome uptake by MSCs. DiO (green lipid dye)-labeled cells were exposed to Dil (red lipid dye)-labeled exosomes for $24 \mathrm{~h}$ and fixed in 4\% PFA. Control cells were treated in the same manner without exosomes. Pictures were acquired by an Operetta high content screening system (Perkin Elmer). The lower two images represent an experiment where nuclei were counterstained with DAPI. Exosomes were detected with a customized version of A-torus wavelet transform and were highlighted with yellow. (b) Quantitative analysis of exosome uptake. The graph shows the number of detected exosomes ( $\mathrm{Y}$ axis) in each cell (X axis) in three exosome-exposed (24 h) and three control cell cultures (ctrl). (c) Cell proliferation assay of exosome-exposed MSCs. Cells were plated at a density of $1 \times 10^{4} \mathrm{cell} / \mathrm{cm}^{2}$ density in control and exosome-exposed cultures, and $72 \mathrm{~h}$ after the exosome treatment, the cell number was determined by manual counting and by an automated cell counter. Both methods showed significantly increased cell proliferation of exosome-treated cells. Results are presented as mean $\pm \mathrm{SD}(\mathrm{n}=3)$. (d) Apoptosis analysis of exosome-exposed MSCs by flow cytometry. Exosomepre-exposed cells were treated by $100 \mathrm{ng} / \mathrm{ml} \mathrm{TNF}-\alpha$ for $24 \mathrm{~h}$, stained with Annexin V-FITC and propidium iodide. TNF- $\alpha$-treated and the untreated control cell cultures were both analyzed by flow cytometry. The graph represents the percentage of early apoptotic, late apoptotic, and necrotic cells. Both TNF- $\alpha$-induced total cell death $(\Sigma)$ and necrosis were significantly lower $(\Sigma p=0.03$ and $p=0.02$, respectively) in exosome-pretreated cell cultures compared to the corresponding control cells. Results are presented as mean +SD (n = 3). (e) qRT-PCR analysis of Mlana and Mitf in MSC cultures treated by exosomes every $24 \mathrm{~h}$ as indicated by arrows in the graph. The expression of both mRNAs increased after exosome exposure, but they showed different kinetics. (f) Fluorescent immunocytochemistry of MLANA in exosome-exposed MSC cultures using a primary rabbit antibody to MLANA and a secondary AlexaFluor555-conjugated antibody to rabbit IgG (red). $\alpha$-tubulin network of cells was directly labeled by an AlexaFluor488-conjugated antibody (green) and the nuclei were stained with DAPI (blue). 
Next, we assessed whether the above effects of exosomes - whereby they induce a malignantlike transformation of the MSCs - was accompanied by a cellular-molecular oncogenic reprogramming of the target cells. Naïve MSCs were exposed to a standardized volume of exosomes for various time intervals (to avoid the experimental fluctuations, we collected pooled samples from multiple independent in vitro experiments). Then, samples were subjected to qRTPCR analysis using a self-designed panel of 40 oncogenes and tumor suppressor genes that were previously suggested to play a role in melanoma progression (Table 1).

Table 1. List of genes investigated by a self-designed oncopanel

\begin{tabular}{|c|c|c|c|}
\hline Gene & Protein & Function & References \\
\hline Alcam & $\begin{array}{l}\text { ALCAM (CD166) } \\
\text { Activated leukocyte cell adhesion molecule }\end{array}$ & $\begin{array}{l}\text { plays an important role in human malignant melanoma progression } \\
\text { and formation of locoregional and distant metastases }\end{array}$ & 78 \\
\hline Bmil & $\begin{array}{l}\text { BMI1 } \\
\text { B cell-specific Moloney murine leukemia virus } \\
\text { integration site } 1\end{array}$ & $\begin{array}{l}\text { induces an invasive signature in melanoma that promotes metasta- } \\
\text { sis and chemoresistance }\end{array}$ & 79 \\
\hline $\mathrm{Cd} 44$ & CD44 & $\begin{array}{l}\text { is a CD44s interaction with HA plays a crucial role in cell inva- } \\
\text { siveness }\end{array}$ & 80 \\
\hline Eng & $\begin{array}{l}\text { ENG } \\
\text { Endoglin (CD105) }\end{array}$ & $\begin{array}{l}\text { has a crucial role in angiogenesis, important protein for tumor } \\
\text { growth, survival and metastasis of cancer cells to other locations } \\
\text { in the body }\end{array}$ & 81 \\
\hline Flot2 & $\begin{array}{l}\text { FLOT1 } \\
\text { Flotillin-2 }\end{array}$ & is associated with melanoma progression & 82 \\
\hline Itga2 & $\begin{array}{l}\text { ITGA2 } \\
\text { Integrin alpha2 }\end{array}$ & is associated with increased risk of melanoma & 83 \\
\hline Itga4 & $\begin{array}{l}\text { ITGA } 4 \\
\text { Integrin alpha } 4\end{array}$ & $\begin{array}{l}\alpha 4 \beta 1 \text { integrin plays an important role in metastasis of malignant } \\
\text { melanoma }\end{array}$ & 83 \\
\hline Itga6 & $\begin{array}{l}\text { ITGA6 } \\
\text { Integrin alpha } 6\end{array}$ & $\begin{array}{l}\alpha 6 \beta 1 \text { integrin as a laminin receptor expression is associated with } \\
\text { invasive potential in a highly metastatic melanoma cell line }\end{array}$ & 83 \\
\hline Itgb1 & $\begin{array}{l}\text { ITGB1 } \\
\text { Integrin beta-1 (CD29) }\end{array}$ & $\begin{array}{l}\alpha 4 \beta 1 \text { integrin plays an important role in metastasis of malignant } \\
\text { melanoma }\end{array}$ & 83 \\
\hline Kit & $\begin{array}{l}\text { KIT (CD117) } \\
\text { Mast/stem cell growth factor receptor (SCFR) }\end{array}$ & c-Kit signaling activates the MAPK and PI3K signaling cascades & 84 \\
\hline Muc1 & $\begin{array}{l}\text { MUC1 } \\
\text { Mucin1 cell surface associated }\end{array}$ & promotes melanoma migration through the Akt signaling pathway & 84 \\
\hline Pecam1 & $\begin{array}{l}\text { PECAM1 } \\
\text { Platelet endothelial cell adhesion mole- } \\
\text { cule (CD31) }\end{array}$ & $\begin{array}{l}\text { can play multiple roles in diverse processes related to melanoma } \\
\text { development, dormancy, migration/invasion and angiogenesis }\end{array}$ & 85 \\
\hline Prom1 & Prominin-1 (CD133) & is a melanoma stem cell marker & 86 \\
\hline Thy & CD90 & $\begin{array}{l}\text { is a cell adhesion molecule. Melanoma cells use Thy- } 1 \text { on endo- } \\
\text { thelial cells for metastasis formation }\end{array}$ & 87 \\
\hline $\mathrm{Cdc} 42$ & $\begin{array}{l}\text { CDC42 } \\
\text { Cell division control protein } 42 \text { homolog }\end{array}$ & $\begin{array}{l}\text { is vital for the transforming Ras signal emanating from endomem- } \\
\text { branes }\end{array}$ & 88 \\
\hline Tiam1 & $\begin{array}{l}\text { TIAM1 } \\
\text { T-cell lymphoma invasion and metastasis } 1\end{array}$ & $\begin{array}{l}\text { has crucial roles in regulation of the actin cytoskeleton, cell migra- } \\
\text { tion, cell cycle progression, gene transcription and cell adhesion }\end{array}$ & 89 \\
\hline
\end{tabular}




\begin{tabular}{|c|c|c|c|}
\hline Gene & Protein & Function & References \\
\hline $\mathrm{Bcl} 2$ & $\begin{array}{l}\text { BCL-2 } \\
\text { B-cell lymphoma } 2\end{array}$ & $\begin{array}{l}\text { plays a pivotal role in the regulation of molecules associated with } \\
\text { the migratory and invasive phenotype, contributing, in cooperation } \\
\text { to hypoxia, to tumor progression }\end{array}$ & 84 \\
\hline Bax & $\begin{array}{l}\text { BAX } \\
\text { Bcl-2-associated X protein }\end{array}$ & $\begin{array}{l}\text { plays a crucial role in apoptotic cell death induced, the Bax/Bcl-2 } \\
\text { ratio determines the susceptibility of melanoma cells }\end{array}$ & 90 \\
\hline Casp9 & $\begin{array}{l}\text { CASP9 } \\
\text { Caspase-9 }\end{array}$ & is linked to the mitochondrial death pathway & 90 \\
\hline Casp8 & $\begin{array}{l}\text { CASP8 } \\
\text { Caspase- } 8\end{array}$ & plays a central role in the execution-phase of cell apoptosis & 91 \\
\hline Cdk4 & $\begin{array}{l}\text { CDK } 4 \\
\text { Cyclin-dependent kinase } 4\end{array}$ & $\begin{array}{l}\text { promotes cell-cycle progression and inhibit both cell senescence } \\
\text { and apoptosis }\end{array}$ & 84 \\
\hline Elk1 & $\begin{array}{l}\text { ELK1 } \\
\text { ETS domain-containing protein Elk-1 }\end{array}$ & is a member of ETS oncogene family, transcription activator & 92 \\
\hline Ets1 & $\begin{array}{l}\text { ETS1 } \\
\text { E26 transcription factor }\end{array}$ & $\begin{array}{l}\text { is required for migration in cell lines with an active RAS/ERK } \\
\text { signaling pathway }\end{array}$ & 84 \\
\hline $\mathrm{Hgf}$ & $\begin{array}{l}\text { HGF } \\
\text { Hepatocyte growth factor }\end{array}$ & $\begin{array}{l}\text { can activate the MAP-kinase pathway, which is upregulated in the } \\
\text { majority of melanoma, through the proto-oncogene c-MET }\end{array}$ & 93 \\
\hline Jak2 & $\begin{array}{l}\text { JAK2 } \\
\text { Janus kinase } 2\end{array}$ & $\begin{array}{l}\text { is an activator of transcription (STAT) pathway is thought to play a } \\
\text { central role in melanoma cell biology }\end{array}$ & 94 \\
\hline Met & $\begin{array}{l}\text { MET } \\
\text { Hepatocyte growth factor receptor }\end{array}$ & $\begin{array}{l}\text { induces several biological responses that collectively give rise to a } \\
\text { program known as invasive growth }\end{array}$ & 95 \\
\hline Myb & $\begin{array}{l}\text { MYB } \\
\text { transcriptional activator Myb }\end{array}$ & $\begin{array}{l}\text { is a transcription factor; among other genes, MYB regulates the } \\
\text { transcription of the Kit, Bcl2, Ets-2 and N-Ras }\end{array}$ & 96 \\
\hline Nras & $\begin{array}{l}\text { NRAS } \\
\text { Neuroblastoma RAS viral oncogene homolog }\end{array}$ & $\begin{array}{l}\text { recruits and stimulates a number of intracellular signaling path- } \\
\text { ways including the Raf/MEK/ERK mitogen activated protein } \\
\text { kinase (MAPK) pathway, the PI3K/AKT pathway }\end{array}$ & 84 \\
\hline Stat3 & $\begin{array}{l}\text { STAT3 } \\
\text { Signal transducer and activator of transcription } 3\end{array}$ & $\begin{array}{l}\text { promotes transcription of many genes that involve in melanoma } \\
\text { metastasis }\end{array}$ & 97 \\
\hline Kitl & $\begin{array}{l}\text { KIT-ligand } \\
\text { Stem cell factor (CD117) }\end{array}$ & $\begin{array}{l}\text { is a cytokine that binds to the c-KIT receptor. This cytokine plays } \\
\text { an important role in melanogenesis }\end{array}$ & 84 \\
\hline $\mathrm{Rb} 1$ & $\begin{array}{l}\text { RB1 } \\
\text { Retinoblastoma } 1 \text { protein }\end{array}$ & $\begin{array}{l}\text { is a tumor suppressor protein that is dysfunctional in several ma- } \\
\text { jor cancers }\end{array}$ & 98 \\
\hline Pik3ca & $\begin{array}{l}\text { PI3K } \\
\text { Phosphatidylinositide 3-kinases }\end{array}$ & $\begin{array}{l}\text { is a PI3K/AKT pathway play a pivotal role in tumor development, } \\
\text { growth and metastasis of melanoma }\end{array}$ & 84 \\
\hline Raf1 & $\begin{array}{l}\text { RAF1 } \\
\text { Proto-oncogene serine/threonine-protein kinase }\end{array}$ & is a crucial regulators of the ERK MAP kinase signaling cascade & 99 \\
\hline Mtor & $\begin{array}{l}\text { mTOR } \\
\text { Serine/threonine-protein kinase }\end{array}$ & $\begin{array}{l}\text { mechanistic target of rapamycin, is a serine/threonine protein } \\
\text { kinase that regulates cell growth, cell proliferation, cell motility, } \\
\text { cell survival }\end{array}$ & 83 \\
\hline Akt1 & $\begin{array}{l}\text { AKT } \\
\text { Protein kinase B }\end{array}$ & $\begin{array}{l}\text { plays a key role in multiple cellular processes such as glucose } \\
\text { metabolism, apoptosis, cell proliferation, transcription and cell } \\
\text { migration }\end{array}$ & 84 \\
\hline Map2k1 & $\begin{array}{l}\text { MEK1 } \\
\text { Dual specificity mitogen-activated protein } \\
\text { kinase kinase } 1\end{array}$ & $\begin{array}{l}\text { is an essential component of the MAP kinase signal transduction } \\
\text { pathway, this kinase is involved in many cellular processes such } \\
\text { as proliferation, differentiation, transcription regulation and devel- } \\
\text { opment }\end{array}$ & 84 \\
\hline
\end{tabular}




\begin{tabular}{llll}
\hline Table 1. Continued & & \\
\hline Gene & Protein & Function & References \\
\hline Map2k2 MEK2 & $\begin{array}{l}\text { Dual specificity mitogen-activated protein } \\
\text { kinase kinase 2 }\end{array}$ & $\begin{array}{l}\text { plays a critical role in mitogen growth factor signal transduction. } \\
\text { It phosphorylates and thus activatesMAPK1/ERK2 and MAPK2/ } \\
\text { ERK3 }\end{array}$ & 84 \\
\hline Mapk3 3 & $\begin{array}{l}\text { ERK1 } \\
\text { Extracellular-signal-regulated kinases }\end{array}$ & $\begin{array}{l}\text { Ras-Erk1/2 is a key regulator pathway in melanoma cell prolifer- } \\
\text { ation. }\end{array}$ & 84 \\
\hline Mapk1 & $\begin{array}{l}\text { ERK2 } \\
\text { Mitogen-activated protein kinase 1 }\end{array}$ & $\begin{array}{l}\text { Ras-Erk1/2 is a key regulator pathway in melanoma cell prolifer- } \\
\text { ation }\end{array}$ & 84 \\
\hline Rac1 & $\begin{array}{l}\text { RAC1 } \\
\text { Ras-related C3 botulinum toxin substrate 1 }\end{array}$ & $\begin{array}{l}\text { functions in multiple signaling pathways are leading to cell adhe- } \\
\text { sion, migration, proliferation and transformation }\end{array}$ & 100 \\
\end{tabular}

As shown in Figure 7a, the gene expression pattern of MSCs exposed to melanoma exosomes exhibited a clear oncogenic dominance (compared to the non-exposed cells). This was verified by statistical analysis of the mean relative gene expression levels of all molecules investigated. Statistically higher values were obtained in the case of exosome-treated cells $\left(\mathrm{p}=1.9 \times 10^{-5}\right.$, $\mathrm{p}=0.031$ and $\mathrm{p}=2.3 \times 10^{-8}$ for the $6 \mathrm{~h}, 24 \mathrm{~h}$ and $72 \mathrm{~h}$ time points, respectively).

As mentioned above, Kleffel et al. has recently shown that melanoma cell subpopulations which overexpress PD-1, quite intriguingly exhibit remarkably increased invasiveness and aggressive growth properties (70). However, the authors did not define the factor(s) which induced the above PD-1 overexpression. Since the above finding strongly suggested an "MSC re-education" capacity of melanoma exosomes to induce malignant-like behavior, we next assessed the expression of PD-1 in MSC cultures.

As expected, only insignificant PD-1 expression (both at the mRNA and protein levels) could be identified in control, non-treated MSCs. In contrast, a marked, significant, and time-dependent elevation of PD-1 expression was detected upon exosome treatment by qRT-PCR (Figure 7b), Western blot and an immunocytochemical analysis (Figure 7c,d). Furthermore, by employing super-resolution microscopy, we were able to identify a dramatic upregulation of PD-1 at the single molecular level in exosome-treated MSCs (Figure 7e). 
a

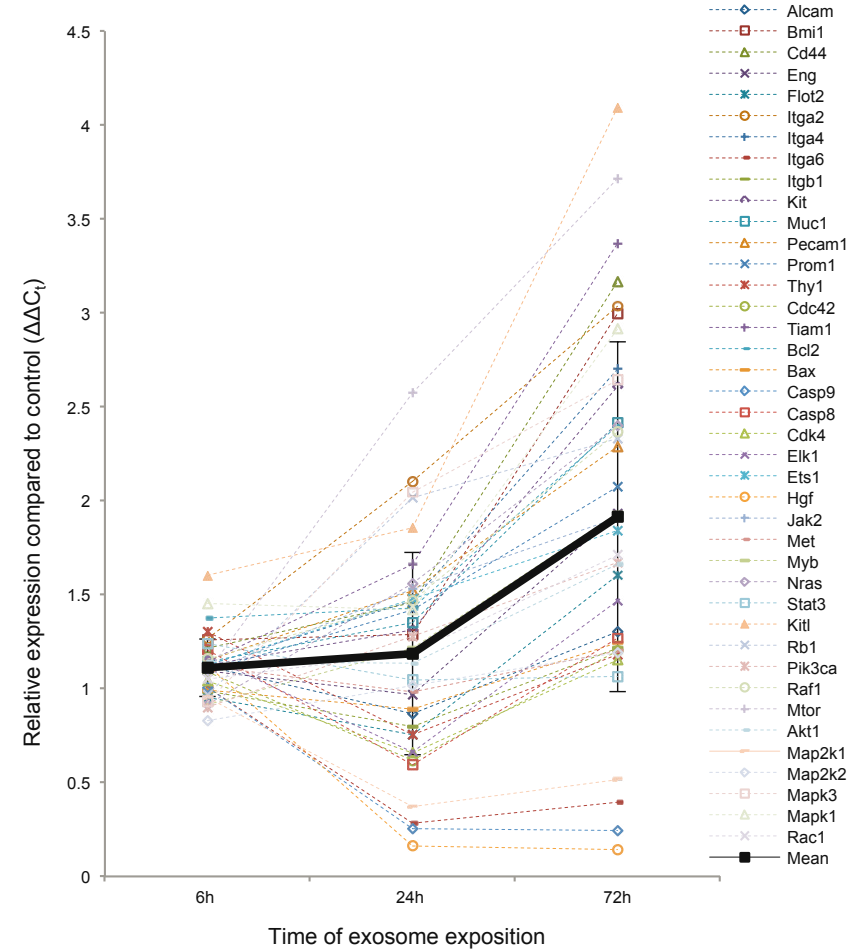

d

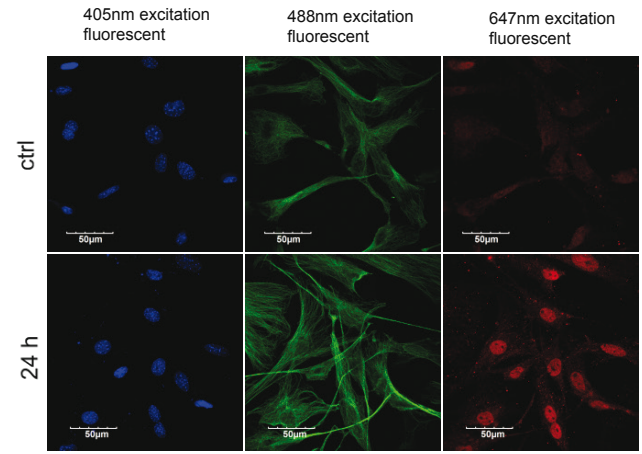

Merged

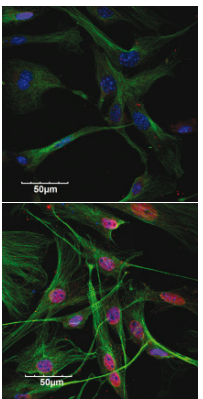

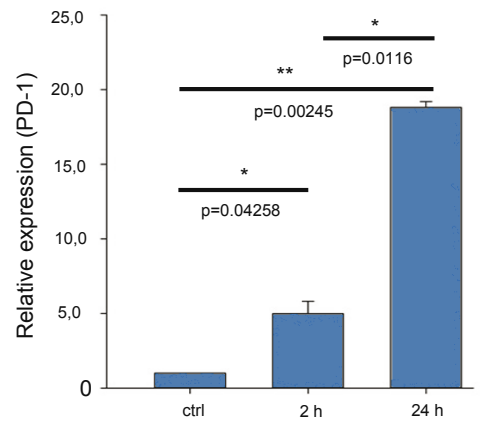

C

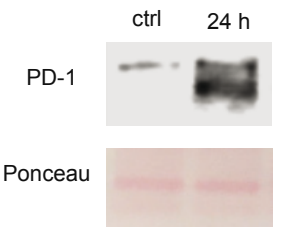

e

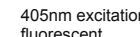

$647 n m$ excitation
STORM

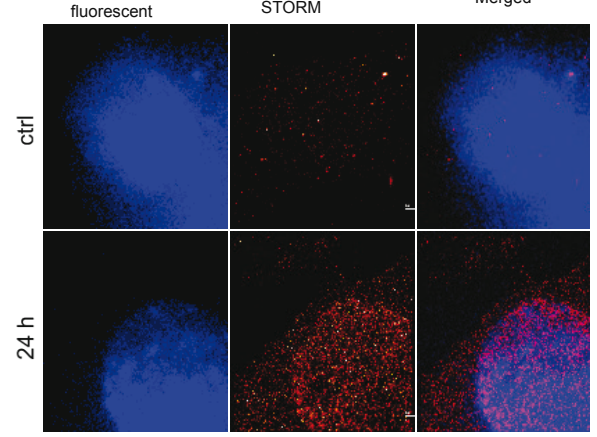

Figure 7. Exosome re-educated MSCs show oncogene dominance and PD-1 expression.

(a) qRT-PCR analysis of 40 tumor-related genes in exosome-exposed MSCs using a self-designed panel. The graph shows the relative expression values for each gene after $6 \mathrm{~h}, 24 \mathrm{~h}$ and $72 \mathrm{~h}$ of exosome exposure. The trend line of the altered gene expression pattern (indicated by thick black line) shows an increasing tendency over time (mean $\pm \mathrm{SD}$ ). (b) qRT-PCR analysis of PD-1 in MSCs after $2 \mathrm{~h}$ and $24 \mathrm{~h}$ of exosome exposure. The graph represents mean + SEM $(n=3)$. (c) Representative immunoblot of PD-1 protein expression in control and exosome-exposed MSCs after $24 \mathrm{~h}$ of exosome treatment. (d,e) Fluorescent immunocytochemistry of PD-1 in $24 \mathrm{~h}$ exosome-exposed MSC cultures, using a primary rat antibody to PD-1 and a secondary AlexaFluor647conjugated antibody to rat IgG (red). Nuclei were stained with DAPI. (d) $\alpha$-tubulin network of cells was directly labeled by an AlexaFluor488-conjugated antibody (green). Images were acquired by confocal microscopy. (e) Images were taken by STORM super-resolution microscopy. STORM super-resolution imaging of PD-1 revealed that PD-1 was localized mostly around the nucleus, which were blurred and were not resolvable using diffractionlimited confocal microscopy (d) of the same region.

Importantly, since proteomics analysis did not identify the presence of PD-1 in exosomes, these data suggest that the high PD-1 protein content in exosome-exposed MSCs was a result of de novo induction and not of exosome-mediated molecular transfer. Our findings therefore 
suggest that melanoma exosome-mediated "re-education" of the cells resulted in a novel MSC population which could be identified as $\mathrm{MSC}^{\mathrm{PD}-1+}$.

\section{B16F1 exosomes augment in vivo tumorigenesis and tumor progression}

After presenting evidence for the in vitro tumorigenic induction potential of exosomes on cultured MSCs, we hypothesized that this phenomenon could be identified in vivo as well. To probe this assumption, we employed the well-known animal model, routinely used in our laboratories, in which tumors, developed mostly in the lungs, are induced in mice by intravenous administration of mouse B16F1 melanoma cells (to the tail vein). These tumor-bearing mice were then administered by buffer, or exosomes isolated from the same B16F1 melanoma cells, or exosome-induced $\mathrm{MSC}^{\mathrm{PD}-1+}$ cells.

Notably, the exosome-related groups (i.e. groups treated with exosomes or $\mathrm{MSC}^{\mathrm{PD}-1+}$ ) were characterized by a markedly increased size of tumor-covered lung tissues (the increase proved to be significant in the $\mathrm{MSC}^{\mathrm{PD}-1+}$ group) (Figure 8a). Of further importance, we also found that in both exosome-related groups, the number of distant metastases were significantly elevated when compared to the control (Figure $8 \mathrm{~b}$ ), and the diameter of blood vessels associated with metastases significantly increased in exosome and $\mathrm{MSC}^{\mathrm{PD}-1+}$ injected groups of tumor bearing mice (Figure S3). Levels of tumor supportive cytokines and chemokines (C5/C5a, TIMP-1, IL-16, IL-1F3, SDF1, CCL5) were elevated in the exosome-related groups (Figure S4). In this animal-model, we have previously observed that these metastases were mostly localized to the ovaries and kidneys (and very rarely to the lymph nodes) of control tumor-bearing animals. However, besides these sites, the presence of exosomes resulted in frequent metastases in the lymph nodes and, as a new location, in the liver. Interestingly, in $\mathrm{MSC}^{\mathrm{PD}-1+}$ treated mice, exosometransformed MSCs could be identified in the paraaortic lymph nodes by FISH (fluorescence in situ hybridization) (Figure 8c) verifying the successful in vivo adherence of $\mathrm{MSC}^{\mathrm{PD}-1^{+}}$cells.

Lung tissues of the different groups were then subjected to in-depth expressional profiling 14 days after injection of exosomes or MSC ${ }^{\text {PD-1+ }}$ cells. Namely, qRT-PCR analysis was performed using a self-designed panel of 40 genes; besides housekeeping genes, we assessed the expression of (i) proto-oncogenes; (ii) genes reportedly involved in malignant transformation; and/or (iii) genes found to be involved in melanoma development and progression.

Hierarchical cluster analysis (HCA) of the gene expression patterns clearly showed a robust proto-oncogenic dominance in lung samples from the exosome-related groups when compared to the control tissues (Figure 8d). Indeed, we identified significant de novo induction of the following genes: Alcam1, Eng, Flot2, Itga4, Itga6, Kit, Pecam1, Prom1, Thy, Cdc42, Tiam1, Bcl2, Bax, Casp9, Casp8, Ets1, Hgf, Jak2, Met, Myb, Map2k1, Map2k2, Mapk1 in both groups 
(23 genes in total) and Elk1, Rb1, Itga2 in the exosome group (26 genes in total). Six additional genes (Cd44, Itgb1, Mucl, Pik3ca, Akt1, and Racl) in the exosome group and an three additional genes (Cd44, Itgb1, Rac1) in the MSC ${ }^{\mathrm{PD}-1+}$ group were identified with at least 10 -fold increases.

a

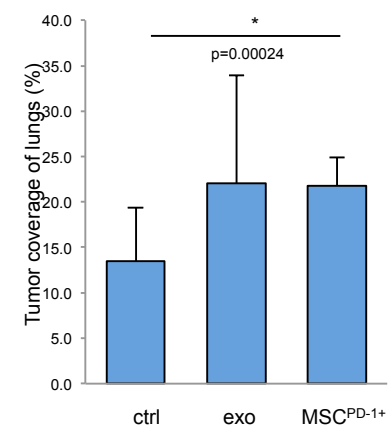

d

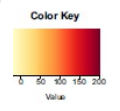

b

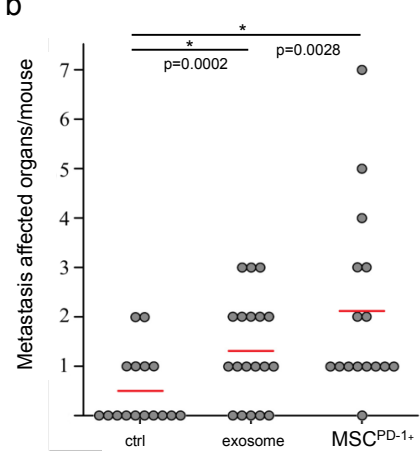

C

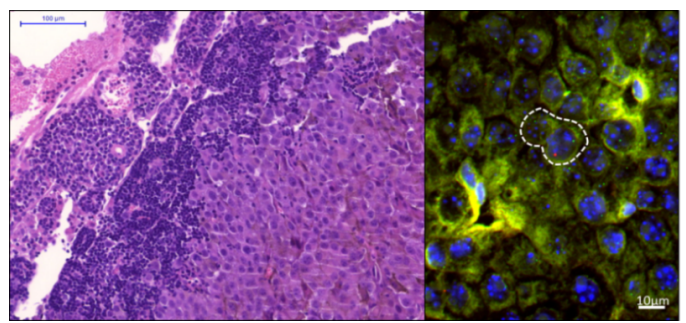

e

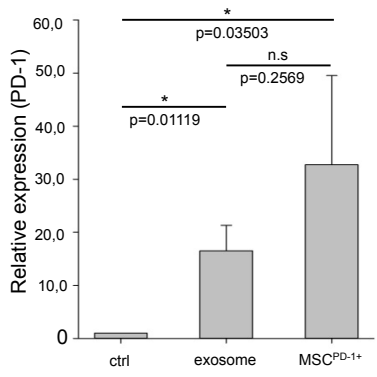

f

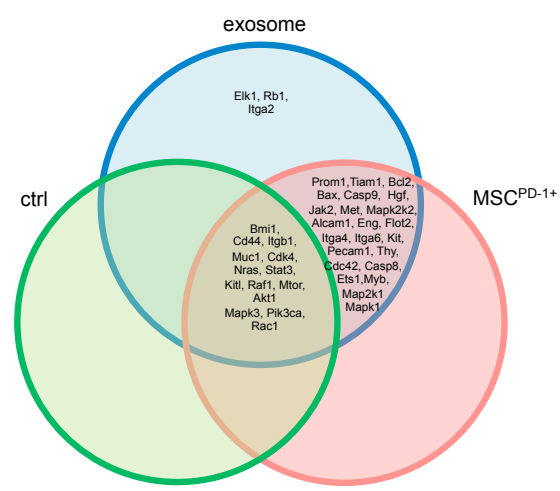

Figure 8. Melanoma exosomes promote tumor progression and metastasis formation in vivo.

(a) Tumor coverage of lungs on day 15 in the three different animal groups. The graph represents mean $+\mathrm{SD}$ $(\mathrm{n}=3)$. (b) The number of distant metastases on day 25. One dot represents one animal in each group, red lines show the average number of metastases per animal. (c) FISH analysis of a paraaortic lymph node metastases, which showed the presence of exosome-exposed MSCs. Y chromosome (red dot) of the male mouse-derived MSC was detected in the metastases of a female mouse. (d) Heatmap and cluster analysis of the gene expression pattern, which show protooncogenic dominance in exosome or $\mathrm{MSC}^{\mathrm{PD}-1+}$ groups. Robust hierarchical clustering based on fold changes in the gene expression data between the selected groups divide the treated groups into several upper classes. Groups treated by exosomes or $\mathrm{MSC}^{\mathrm{PD}-1+}$ were compared to untreated controls. Exosomes and $\mathrm{MSC}^{\mathrm{PD}-1+}$ treatments were closely related. (e) qRT-PCR analysis of PD-1 in the lung samples using TaqMan probes $(\mathrm{n}=3)$. (f) The Venn diagram shows possible relations between a finite collection of different sets of genes measured during gene expression profiling (see Figure 8d). 
Furthermore, six genes (Bmil, Cdk4, Stat3, Kitl, Raf1, Mtor) for the $\mathrm{MSC}^{\mathrm{PD}-1+}$ group and eight genes (Bmi1, Muc1, Cdk4, Stat3, Kitl, Pik3ca, Mtor, Akt1) for the exosome group showed at least two-fold mRNA level elevations. Figure $8 \mathrm{~d}$ presents a heatmap of overexpressed genes induced by exosome exposure.

Moreover, we showed that, besides the above genes, expression of PD-1 was also significantly increased in both exosome-related groups (Figure 8e). Notably, although mRNA transcript level of PD-1 was close to two-fold in $\mathrm{MSC}^{\mathrm{PD}-1+}$ lung tissues in comparison to samples in the exosome group, the difference was not significant (most probably due to the large inter-animal variability and standard error).

We constructed a Venn-diagram (Figure 8f) to show all possible logical connections between the various gene expressions patterns presented in Figure 8d. Importantly, according to cluster analysis, we could not identify a single gene which was missing from the exosome-related groups in comparison to the control tumor-bearing mice. In other words, whereas the exosomerelated mice exhibited gene expression profiles specific to the group, such individual profiles could not be detected in the control group.

Specifically, the following gene expression patterns were defined:

- three genes (Elk1, Rb1, Igta2) were exclusively induced only in the exosome-treated group;

- twenty-three genes (Prom1, Tiam1, Bcl2, Bax, Casp9, Hgf, Jak2, Met, Mapk2k2, Alcam1, Eng, Flot2, Itga4, Itga6, Kit, Pecam1, Thy, Cdc42, Casp8, Ets1, Myb, Map2k1, Mapk1) were found to be upregulated both in the exosome group and the $\mathrm{MSC}^{\mathrm{PD}-1+}$ cell treated group;

- fourteen genes (Bmi1, Cd44, Itgb1, Muc1, Cdk4, Nras, Sat3, Kitl, Raf1, Mtor, Akt1, Mapk3, Pik3ca, Racl) were found to be upregulated in all three groups.

Finally, it should be noted that the dramatic gene expression alterations seen in the exosomerelated groups were exclusively due to the presence of the exosomes as the "MSC ${ }^{\mathrm{PD}-1^{+}}$only" cluster contained no genes (Figure 8f).

\section{Melanoma-derived exosomes promote tumorigenic and cell survival signaling pathway(s)}

Figure 9 shows the map of interaction pathways based on the overexpressed molecules (red symbols) from the in vivo experiments. We detected overexpressed elements from three main pathways which participate in tumor progression and metastasis formation (101). 


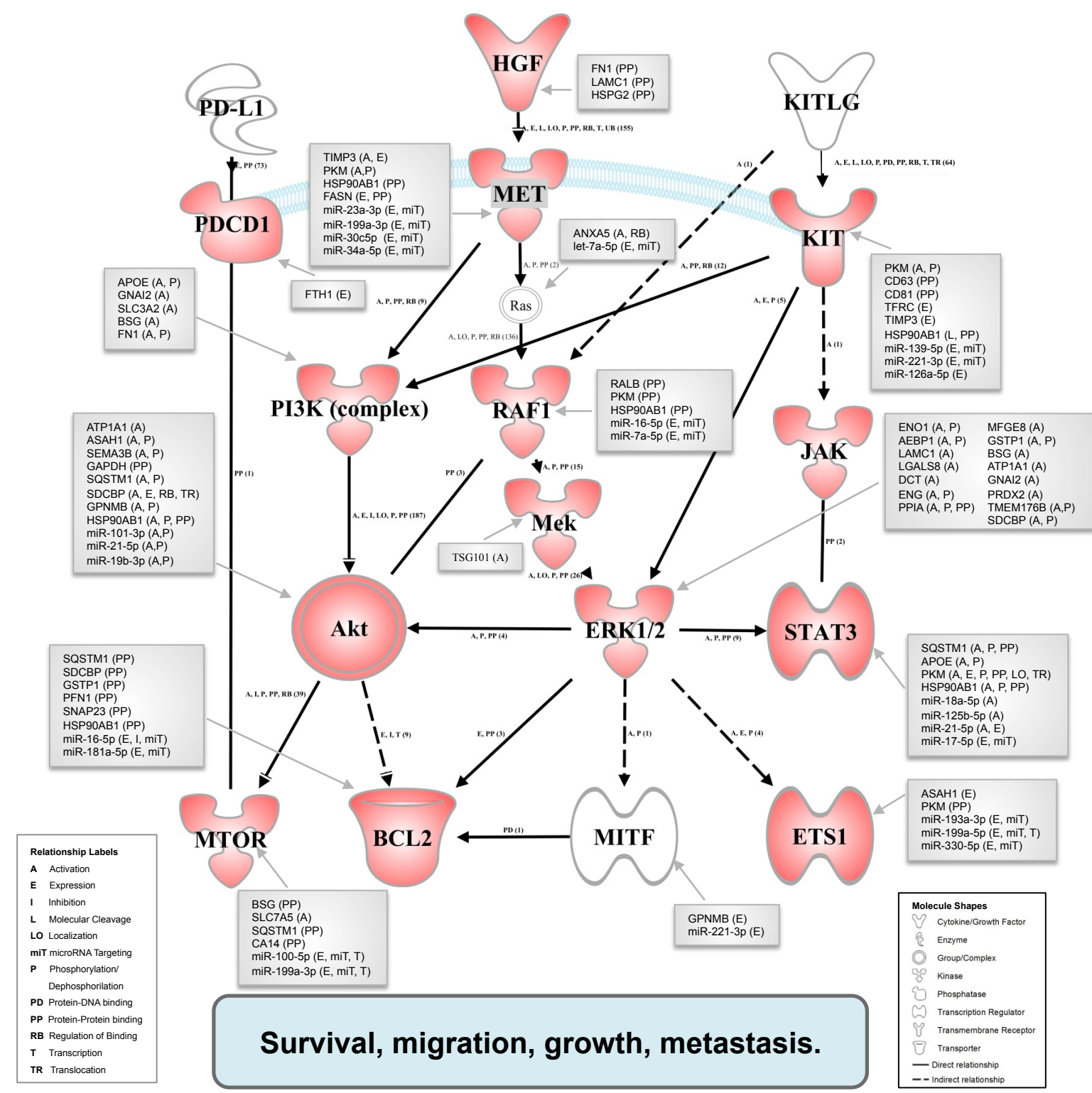

Figure 9. An integrated associative network of the in vivo overexpressed genes supplemented with the interacting exosomal factors. We created this network of overexpressed genes (red symbols) based on literature data. The relationships between molecules were also supported by the IPA knowledge base. Network visualization was performed using the Path Explorer tool of the IPA Path Designer. The exosomal proteins and miRNAs (grey boxes) were connected to elements of the network by the Grow tool of the IPA Path Designer based on experimental data from the IPA knowledge base. Activation of the established network by exosomal components may support the survival, migration, growth and metastasis of tumor cells.

Using the IPA Path Designer Grow tool, we generated an interaction map which contains proteins (or their established complexes) encoded by the overexpressed genes, and exosomal miRNAs and proteins (grey boxes) which were previously shown to control or affect the marked signaling molecules.

To demonstrate these results and to identify potential causative exosomal factors, we performed an IPA again. The analysis allowed further refinement of the underlying molecular processes 
and pathways involved in the summarized effect of this gene expression profile. The previously described 40-gene qRT-PCR panel is abundant in genes related to cellular movement and migration, cell survival and connective tissue development and function, involving tumor cells. Additional processes included growth and proliferation of tumor cells as well as the PD-1:PD-1L interaction (and its consequences). Focusing on the PD-1 and mTOR pathways, a network was built from the overexpressed genes and their complexes known to be related to tumor progression using the Path Explorer tool in the IPA Path Designer. After that, a list was generated that contained the exosomal proteins detected by MS, and the exosomal miRNAs identified by SOLiD sequencing. The Grow tool in the IPA Path Designer revealed significant interactions between the exosome-induced expression network and the molecular content of these vesicles. The resulting interaction network shows both direct and indirect interactions, but it contains only experimentally observed relationships based on the Ingenuity Knowledge Base. This network demonstrates that 61 exosomal molecules (Table S5,S6) may affect tumor progression through pathways controlled by key components including MET, RAS, RAF1, MEK, ERK1/2, MITF, BCL2, PI3K, AKT, mTOR, PD-1, KIT, JAK STAT3 or ETS1.

Taken together, these findings demonstrate that the interaction between exosomes and MSCs induces a tumor-like phenotype with PD-1 overexpression of naïve MSCs in vitro and a fast tumor progression in vivo.

On the other hand, we investigated the melanoma TME on the attitude of tumor-infiltrating immune cells and we demonstrate that bacterial infection may induce a complex anti-tumor immune response.

\section{Treatment of immunocompetent mice with $C$. pneumoniae reduces the number of lung metastases and increases survival}

To clarify the role of the tumor associated macrophages in the anti-tumor immune mechanisms induced by C. pneumoniae, metastatic melanomas were generated in immunocompetent C57BL/6 mice or immunodeficient NSG mice. B16F1 melanoma cells were administered intravenously to 6 to 8-week old female immunocompetent C57BL/6 or immunodeficient NSG mice. One week after tumor cell administration, the mice were treated with heat-inactivated C. pneumoniae or the mock as a negative control. In immunocompetent animals, the number of lung metastases significantly decreased ( $\mathrm{p}=0.003 ; 3$ independent experiments) in the inactivated $C$. pneumoniaetreated group vs. mock (Figure 10a). Moreover, the survival of the inactivated C. pneumoniaetreated mice (Figure 10b) was significantly increased ( $\mathrm{p}=0.04 ; 3$ independent experiments). Importantly, such phenomena were not observed in immunodeficient mice suggesting the antitumor role of the immune response. It is also notable that the treated animals (in either strain) 
did not have fever $\left(33.2^{\circ} \mathrm{C} \pm 1.0\right.$ control vs. $34.8^{\circ} \mathrm{C} \pm 0.5$ treated $)$ which is against the "fever hypothesis" (33) according to which the fever induction may be considered as part of a Coley's immunological therapy regimen for cancers.

a

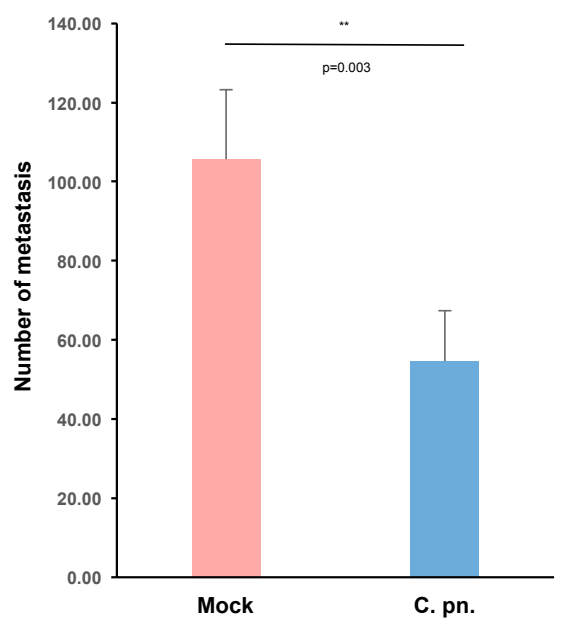

b

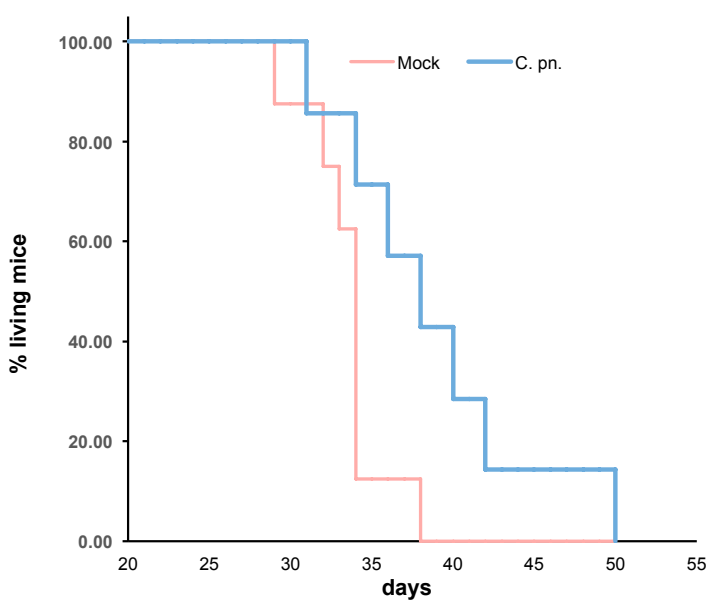

Figure 10. C. pneumoniae treatment results in melanoma metastasis regression and increases survival of animals. (a) The number of lung metastases and (b) survival rate of mock or C. pneumoniae (C. pn.) treated immunocompetent C57BL/6 mice.

\section{C. pneumoniae treatment induces tumor regression and immune cell infiltration}

Histological sections of lungs from mock-treated melanoma-bearing immunocompetent C57BL/6 mice showed high number of metastases, with frequent intra-tumor necrosis indicating high tumor burden and tumor mass replacing the normal lung tissue (Figure 11a). In contrast, fewer and smaller foci of regressive metastases (with residual pneumonitis) were observed in the $C$. pneumoniae-treated immunocompetent animals (Figure 11b). Moreover, in this group, a high number of tumor infiltrating mononuclear histiocytes and lymphoid cells were identified in the regressive metastases compared to mock-treated samples. Notably, in both mock and C. pneumoniae-treated immunodeficient NSG mice, the abundant metastases showed mostly subpleural and intraparenchymal localization and no significant intratumor immune reactions (Figure 11c,d). 
C57BL/6

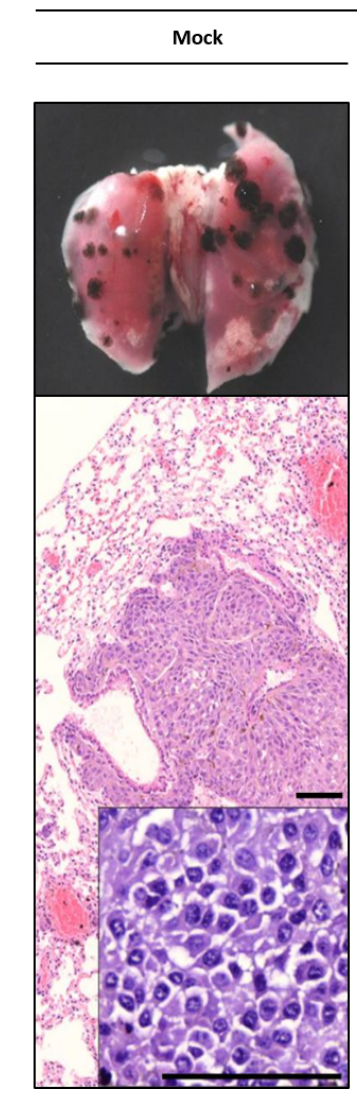

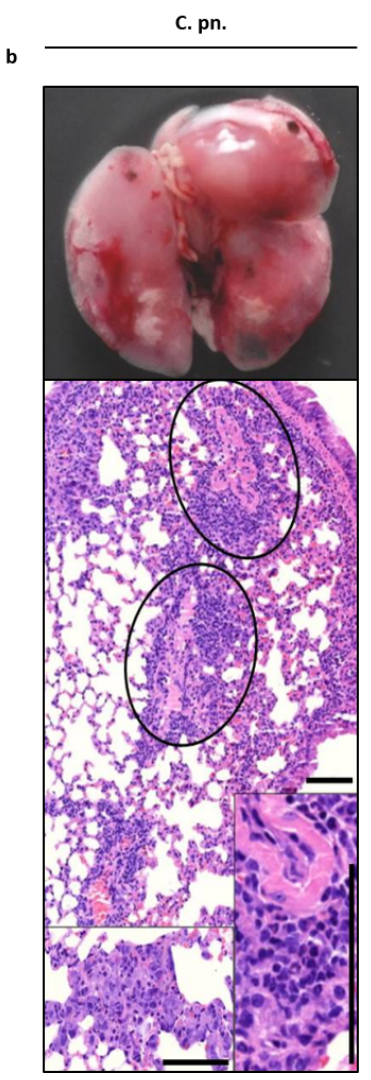

NSG

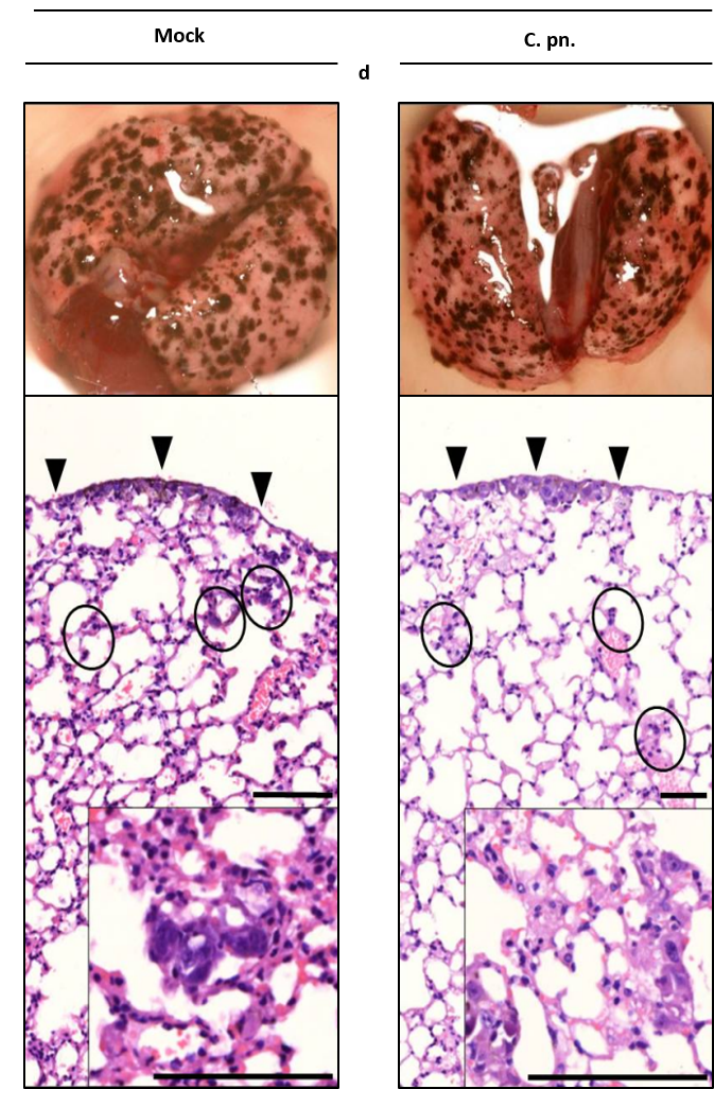

Figure 11. C. pneumoniae treatment induces tumor regression and immune cell infiltration

Representative photos and HE-stained histological sections of dissected lungs of mock (a) and C. pneumoniae (b) treated immunocompetent mice as well as of mock (c) and C. pneumoniae (d) treated immunodeficient (NSG) animals. Scale bars, $100 \mu \mathrm{m}$. (a) Trophical necroses indicating high tumor burden. Insert: atypical tumor cells and regions of necrosis. (b) Circles and right insert, foci of regressive metastases, left insert: areas of residual pneumonitis after $C$. pneumoniae treatment. (c, d) In both mock and C. pneumoniae treated NSG mice, military metastases were developed subpleurally (arrowheads) and intraparenchymally (circles) without significant inflammatory reactions (inserts: higher magnification of intraparenchymal metastases).

The markedly increased immune reaction in the lungs of the C. pneumoniae-treated melanomabearing C57BL/6 mice was also verified by immunolabeling of cell surface activation markers CD11b and CD80 (Figure 12). Invasion of immune cells was not detected in the control group where the immune cells were chiefly concentrated in the marginal zones of the tumors (Figure 12a,c). However, dramatically increased numbers of tumor-infiltrating, activated (i.e. CD11b and CD80 positive) lymphocytes were detected within the internal tumor stroma of the C. pneumoniaetreated animals (Figure 12b,d); the differences show high levels of significance in both cases (Figure 12e,f). C. pneumoniae treatment induces M1 type macrophage polarization. 
Mock
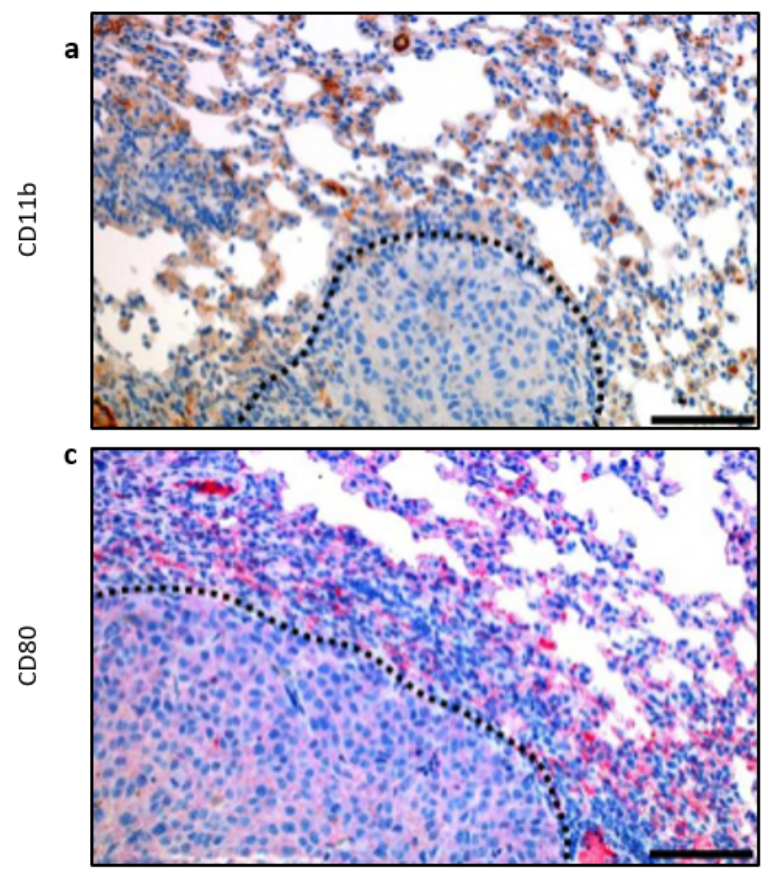

e

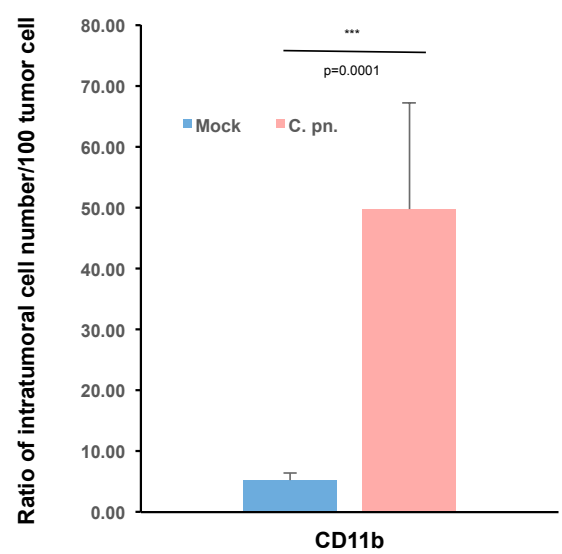

C. pn.
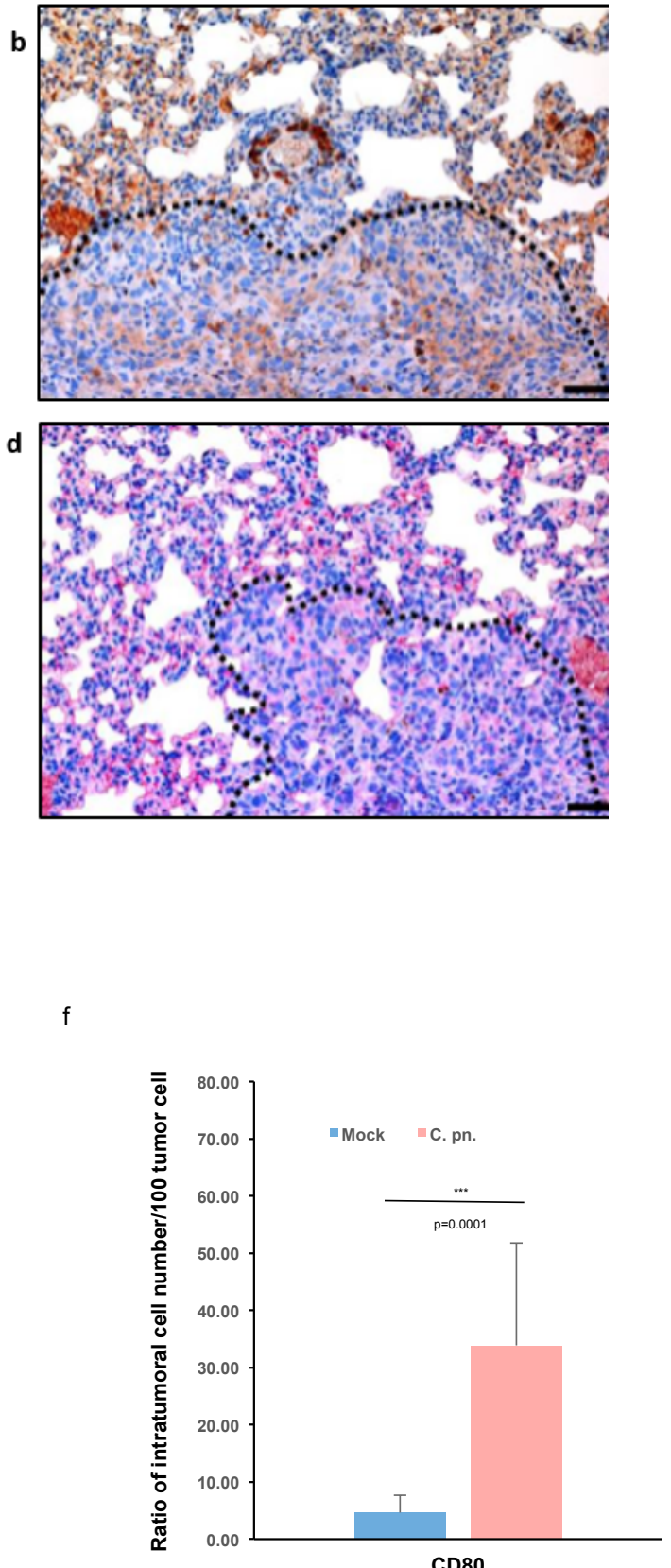

Figure 12. C. pneumoniae treatment results $\mathrm{CD11b}+$ and $\mathrm{CD80}+$ immune cell infiltration of tumor tissues Immunohistochemistry of CD11b (a, b, DAB, brown) and CD80 (c,d, Fast red) on lungs of mock (a, e) or C. pneumoniae (b, d) treated C57BL/6 mice. Dashed lines indicate tumor border. (a-d). Intratumoral number of $\mathrm{CD} 11 \mathrm{~b}+(\mathbf{e})$ and $\mathrm{CD} 80+(\mathbf{f})$ cells determined as a ratio of 100 tumor cells. (e, f) Data are expressed as mean + SD. 


\section{The $C$. pneumoniae treatment induces the macrophage differentiation shift toward an M1 phenotype}

As a next step, we assessed whether C. pneumoniae treatment induced macrophage polarization. To this end, we mapped the cytokine and chemokine transcriptome profiles, characteristics for M1 (anti-tumor) or M2 (protumor) type macrophages $(102,103)$ of tumor bearing immunocompetent mice. M1 and M2 type macrophage markers were detected with qRT-PCR from pooled lung samples (3 animals/pool) 2, 4 and 12 hours after mock or $C$. pneumoniae treatment. qRT-PCR was performed using M1 (CCL2, CCL3, CD86, IL-12, IL-6, IL-10, CXCL16, CCL7, CD80, CXCL11, CXCL9, IL-23, TNF- $\alpha$ ) and M2 type (CD163, CXCL13, TGF- $\beta$, IL-1Ra, CD23, CCL1, CCL22, IL-4, CCL17, CCL24, CD150, IL-10, CXCL1) markers. Four hours after the treatment with $C$. pneumoniae, markedly increased levels of specific mRNA transcripts for CCL2, CCL3, IL-6, CXCL10, CCL7, CD80, CXCL11, CXCL9, IL-23, and TNF- $\alpha$ - markers of M1 type macrophage polarization - were detected (Figure 13a). In line with these data, mRNA expression of CD163, CCL1, TGF- $\beta$, and IL-10 (known M2 type macrophage markers) was decreased by the treatment; yet, levels of other important M2 markers (CXCL13, IL-1Ra) were increased (Figure 13b). Significantly, statistical analysis revealed that upon C. pneumoniae administration, the amount of M1 type cytokine and chemokine-specific mRNA transcripts significantly increased $(p=0.014$ ) (Figure 13a) after 4 hours in comparison to M2 type macrophage markers (Figure 13b).

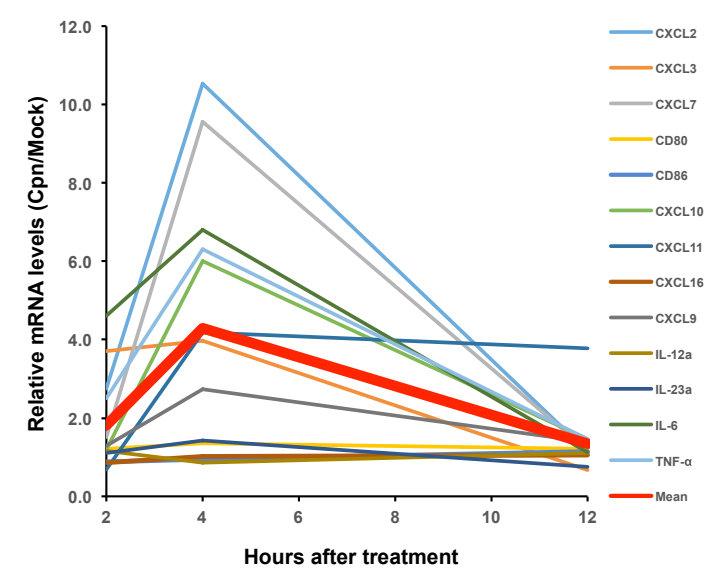

b

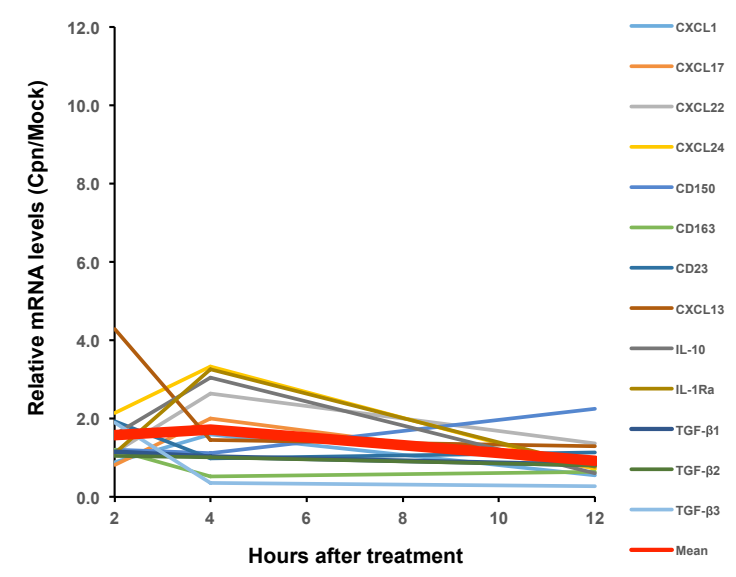

Figure 13. $C$. pneumoniae treatment induces M1 type macrophage polarization

(a) Relative alterations in the levels of individual M1 type cytokine/chemokine specific mRNA transcripts in lung samples of $C$. pneumoniae (C. pn.) vs. mock-treated tumor-bearing C57BL/6 mice, as determined by real-time PCR. (b) Mean values of relative M2 type cytokine mRNA expressions; at 4 hours after treatment, M1 and M2 levels are significantly different (two-tailed t-test). 


\section{DISCUSSION}

It is obvious now that the biology of cancer can no longer be understood simply by specifying the traits of the cancer cells. The contribution of the "tumor microenvironment" to tumorigenesis must also be incorporated into this understanding. Exosomes produced by tumor cells in the TME emerge as a new means of communication operating between cells engaged in supporting tumor progression.

In this study, we aimed to investigate how TME factors influence the pro-metastatic characteristics of melanoma. The TME was investigated from two different points of view; first, the aspect of tumor, and second, the attitude of tumor infiltrating immune cells. We hypothesized that tumor-derived exosomes promote the tumor progression, while the M1 polarization of tumorassociated macrophages induces tumor regression.

As a first step, we intended to precisely define the EV population which we later used in in vitro and in vivo experimental approaches. Besides applying the experimental requirements of the International Society for Extracellular Vesicles (ISEV) (i.e. determination of shape, size distribution and protein markers) (52), we thoroughly defined the miRNA and protein content of melanoma exosomes. Findings of these systematic assays showed us that the isolated vesicles contain factors that may regulate signaling pathways related to cell death and survival, cellular movement, development and cell proliferation. Given the appropriate uptake of these vesicles by the targeted, multipotent recipient cells, it is highly probable that most of these signaling pathways are significantly affected. In accordance with previous results in the literature, we showed successful internalization of exosomes by recipient MSCs. As expected, following $72 \mathrm{~h}$ of exposure, MSCs displayed accelerated proliferation presumably initiated by the transforming exosome cargo. Moreover, melanoma exosomes were able to hinder the induction of the cell death response commonly triggered by TNF- $\alpha$. TNF- $\alpha$ is a soluble tumor-suppressing factor frequently identified in cancerous microenvironments (104). This inhibition strongly suggested that exosomes initialize and sustain the malignant transformation of MSCs.

Furthermore, we described numerous, exosome-induced phenotypic manifestations of the malignant re-education process in the recipient cells addressed above. Apart from the tumor 
markers that were identified, the transformation of exposed cells is also characterized by considerably modified gene expression patterns. As discussed previously, specific gene expression alterations are often associated with specific steps of the cancerous transformation. Altered gene expression in malignant cell lines is responsible for apoptotic regulation (BAX, BCL2, caspases), regulating metastasis (integrins, ITGA2-6, ITGB1, KIT) or various growth factors (ETS1, HGF, MET, etc.). Importantly, we successfully identified two melanoma-specific markers, MITF (Microphthalmia-associated transcription factor) and MLANA (Melanoma antigen recognized by T-cells), in the exosome-exposed MSCs. MLANA and its regulator MITF play a fundamental role in melanocyte development, tumor progression and they were overexpressed in melanoma cells (105). The presence of these markers in exosome-exposed MSCs shows effective transfer of the encoded tumorigenic information, and this information may have a pivotal role in disease progression. In addition, we also identified a characteristic set of genes, which belong mostly to proto-oncogenes, whose expression was significantly elevated upon exosome treatment. Among these molecules, we highlight the increased expression of CD44, which was previously assigned as cancer stem cell marker, and mTOR, a characteristic indicator of the engagement of the PD-1:PD-1L pathway (70). Indeed, the activation of mTOR signaling is particularly important. As it was previously demonstrated by Kleffel et al., overexpression of PD-1 in exceptionally aggressive melanoma subpopulations facilitated tumor progression by activating the mTOR signaling pathway (actually, this response was highly unexpected compared to that of the classic PD-1:PD-L1 dependent T-cell anergy, a crucial aspect of immunotherapy in the clinics) (70). Quite strikingly, in exosome treated MSCs, we detected a significant induction of PD-1 and mTOR. These novel data strongly support that these vesicles might trigger the formation of an aggressive, melanoma-like subpopulation of re-educated recipient MSCs.

Therefore, our findings indicate that the re-educated melanoma-like $\mathrm{MSC}^{\mathrm{PD}-1+}$ subpopulation, originating from multipotent tissue-derived MSCs, is an autonomous entity. It is characterized by typical properties of cancerous transformation, such as hyperproliferation, resistance to apoptosis, expression of melanoma markers, proto-oncogenic gene expression patterns, and PD-1 overexpression. Indeed, the melanoma exosomes that induced in vitro generation of the melanoma-like $\mathrm{MSC}^{\mathrm{PD}-1+}$ cells, also facilitated in vivo metastasis formation in the lungs or other distant organs of treated animals. Actually, our finding, which shows that melanoma exosomes or exosome-re-educated $\mathrm{MSC}^{\mathrm{PD}-1+}$ promote tumor progression, is in good accordance with the previous finding of Kleffel et al., which demonstrates that shRNA or antibody mediated inhibition of PD-1 signaling inhibits metastasis formation and tumor progression in experimental animals (70). Assessment of the expression patterns of genes related to tumor progression in the exposed animal tissues revealed numerous cases of exosome-induced overexpression; this supports our hypothesis that melanoma exosomes should be considered as oncosomes (57). 
Our results also demonstrate that melanoma exosomes also generated a characteristic signaling pattern in the $\mathrm{MSC}^{\mathrm{PD}-1+}$ recipient population. As it was shown in numerous studies, PI3K/ AKT, RAS/MAPK, and STAT3 pathways are commonly activated in tumor cells $(101,106)$. In this study, based on our in vitro and in vivo results and literature data, we introduced a novel, comprehensive network of common tumor-related proteins, such as PD-1, MET, RAF1, STAT3, BCL2 or mTOR. The network highlights the relationship of these elements, and also contains upstream exosomal regulating factors which may contribute to the activation of tumorigenic signaling; thus, fast tumor progression. According to our best knowledge, this associative network between overexpressed genes and the potential exosomal inducers, is the first tumor progression signaling pattern connecting experimental response patterns with experimentally detected exosomal-molecular-patterns. Moreover, our data also indicate that the above complexity of exosomal communication requires system-level approaches.

It is important to note that our conclusions are not based on in silico predictions exclusively, but rather on carefully designed and systematically executed in vitro and in vivo experiments, which all suggest that the specific, robust molecular content of the isolated exosomes can indeed generate a unique intercellular niche responsible for the re-education of neighboring cells via oncogenic transformation. These re-educated melanoma-like $\mathrm{MSC}^{\mathrm{PD}-1+}$ cells, in turn, facilitate metastatic disease progression by initiating a complex series of subsequent events both locally and systematically. Thus, based on our results and recently published, additional evidences about exosomes, there is an urgent demand to supplement and extend the outdated "seed and soil" hypothesis regarding the oncosome-driven re-education process.

On the other hand, it is generally accepted that anti-tumor immune effector mechanisms overlap with antibacterial immune responses (107). Coley's hypothesis claims that fever induced by infectious agents causes tumor regression, but the exact anti-tumor mechanism induced by microbes is not yet understood (108). With respect to anti-tumor mechanisms, it is also known that alterations in the polarization patterns of the overall immune system determine the outcome of malignant diseases (102). Macrophages are key players of the immune responses whose M1 type polarization mediates the anti-tumor action of the immune system $(102,103)$.

In this study, we demonstrated that the heat-inactivated bacterial treatment may induce a complex anti-tumor immune response. The $C$. pneumoniae treatment suppressed metastasis formation (and hence prolonged survival) in immunocompetent, but not in immunodeficient mice. As shown by the cytokine/chemokine profiles, the $C$. pneumoniae treatment induced M1 type macrophage polarization, which, as reported in numerous publications, may exert anti-tumor effects (it must be added that the whole spectrum of M1 polarization was not observed) (102). The anti-tumor immune polarization/activation was further verified by detecting the profound 
enrichment of CD80 and CD11b expressing immune cells in the lungs of C. pneumoniae-treated animals. Indeed, it was previously shown that $C$. pneumoniae, recognized by TLR2 and TLR4, upregulates the expression of the immune activation marker CD11b and the costimulatory molecules CD40, CD80 and CD86 on macrophages (109).

These data prompted us to hypothesize that the concomitant anti-microbial immune response may contribute to the anti-tumor effect.

\section{NEW FINDINGS}

1. Internalized melanoma-derived exosomes conveying oncogenic molecular reprogramming induce the malignant transformation of the recipient MSCs.

2. The melanoma-derived exosomes generate the formation of a melanoma-like, PD-1 overexpressing cell population ( $\mathrm{mMSC}^{\mathrm{PD}-1+}$ ) from naïve mesenchymal stem cells (MSCs).

4. Melanoma-derived exosomes and the re-educated melanoma-like $\mathrm{MSC}^{\mathrm{PD}-1+}$ cell population induce tumor progression and facilitate metastatic disease via overexpression of oncogenic factors in vivo.

5. Bacterial antigen pattern-induced macrophage polarization and complex anti-tumor immune response indeed leads to the regression of melanoma metastasis.

\section{CONCLUSIONS}

We aimed to understand how the tumor microenvironment impacts tumor progression and metastatic behavior. Most of the efforts in the past focused on defining intrinsic and extrinsic factors involved in tumor progression and metastasis. However, the interpretation of tumor evolution and metastasis has shifted and currently, it is widely accepted that extrinsic factors are actively involved in cancer progression and regression. To answer this question, we used melanoma models to investigate the role of secreted exosomes in tumor microenvironment education and pre-metastatic niche formation. In addition, we explored the influence of bacterial antigens on macrophage polarization in anti-tumor immune effector mechanisms. 
In this study, we demonstrated that MSCs, widely abundant in solid tumors as well as in healthy tissues, undergo a marked re-education process upon communication with metastatic cancer cells via exosome-mediated information transfer. This transformation process results in characteristic response patterns corresponding to a given cancerous cell line. We (i) determined the cellular and molecular signals involved in the melanoma-derived exosome-induced, malignant transformation of MSC culture; (ii) described the melanoma-derived exosome-induced tumor progression; (iii) demonstrated the alteration in the expression of PD-1, a melanoma progression marker, and therapeutic target, upon exposure to melanoma-derived exosomes.

Metastasis, a critical phase of melanoma progression, remains a major cause of melanoma mortality and primary challenge in therapy. In this study, we highlighted the emerging roles of melanoma exosomes as coordinators of PMN formation.

Our experimental data provide compelling evidence for the significant tumor regression following the treatment of melanoma lung metastases with the inactivated intracellular pathogen C. pneumoniae, and suggest that this microbe may be responsible for tumor regression via the induction of anti-tumoral macrophage polarization. 


\section{ACKNOWLEDGEMENT}

I would like to express my deep and sincere gratitude to my supervisor and friend, Krisztina Buzás, for her excellent scientific guidance, continuous motivation, patience, unconditional trust, and for her unwavering faith. This thesis would not have been possible without her help and support.

I am grateful to the head of the Institute of Biochemistry and the Laboratory of Image Analysis and Machine Learning in the BRC, Péter Horváth, for the scientific and infrastructural support of my project.

I am grateful to my colleagues Mária Harmati, Gabriella Dobra, Lilla Pintér, Mátyás Bukva for the scientific and technical aid, and for creating a great atmosphere in the laboratory. I would like to thank all help for the other members of the Laboratory of Image Analysis and Machine Learning.

I wish to thank Annamária Marton, Csaba Vizler, Katalin Jósvay for the scientific and technical support.

I also thank all of the co-authors of my publications for their helpful cooperation, and support. I would like to extend my special thanks to Péter Závodszky, Péter Gál, Andrea Kocsis, Júlianna Barlczerné, József Dobó, István Hajdú, Barbara Végh, László Barna, László Beinhror, my colleagues at the Laboratory of Structural Biophysics for their guidance in the complex world of structural biochemistry, their patience, and their support.

The research was supported by GINOP-2.3.2-15-2016-00015; GINOP-2.2.1-15-2017-00052, NKFI-6-K-11493, János Bolyai Research Scholarship of the Hungarian Academy of Sciences, and ÚNKP-19-4.

Lastly, but most importantly, I wish to thank my parents and my brother for their unconditional care, and support throughout my life; my family - who missed me a lot during the last years for their support, encouragement, and patience. 


\section{REFERENCES}

1. Hanahan D, Weinberg RA. The hallmarks of cancer. Cell. 2000;100(1):57-70. doi: 10.1016/ s0092-8674(00)81683-9

2. Hanahan D, Weinberg RA. Hallmarks of cancer: the next generation. Cell. 2011;144(5):646674. doi: 10.1016/j.cell.2011.02.013

3. Quail DF, Joyce JA. Microenvironmental regulation of tumor progression and metastasis. Nat Med. 2013;19(11):1423-1437. doi: 10.1038/nm.3394

4. Polyak K, Haviv I, Campbell IG. Co-evolution of tumor cells and their microenvironment. Trends Genet. 2009;25(1):30-38. doi: 10.1016/j.tig.2008.10.012

5. $\mathrm{Hu}$ M, Yao J, Carroll DK, et al. Regulation of in situ to invasive breast carcinoma transition. Cancer Cell. 2008;13(5):394-406. doi: 10.1016/j.ccr.2008.03.007

6. Sica A, Larghi P, Mancino A, et al. Macrophage polarization in tumour progression. Semin Cancer Biol. 2008; 18(5):349-355. doi: 10.1016/j.semcancer.2008.03.004

7. Finger EC, Giaccia AJ. Hypoxia, inflammation, and the tumor microenvironment in metastatic disease. Cancer Metastasis Rev. 2010;29(2):285-293. doi: 10.1007/s10555-0109224-5

8. Korkaya H, Liu S, Wicha MS. Breast cancer stem cells, cytokine networks, and the tumor microenvironment. J Clin Invest. 2011;121(10):3804-3809. doi: 10.1172/JCI57099

9. Lazennec G, Jorgensen C. Concise review: adult multipotent stromal cells and cancer: risk or benefit?. Stem Cells. 2008;26(6):1387-1394. doi: 10.1634/stemcells.2007-1006

10. Ridge SM, Sullivan FJ, Glynn SA. Mesenchymal stem cells: key players in cancer progression. Mol Cancer. 2017;16(1):31. doi: 10.1186/s12943-017-0597-8

11. Suzuki K, Sun R, Origuchi M, et al. Mesenchymal stromal cells promote tumor growth through the enhancement of neovascularization. Mol Med. 2011;17(7-8):579-587. doi: 10.2119/molmed.2010.00157

12. Mishra PJ, Mishra PJ, Glod JW, Banerjee D. Mesenchymal stem cells: flip side of the coin. Cancer Res. 2009;69:1255-8. doi: 10.1158/0008-5472.CAN-08-3562

13. Sethi N, Kang Y. Unravelling the complexity of metastasis - molecular understanding and targeted therapies. Nat Rev Cancer. 2011;11(10):735-748. doi: 10.1038/nrc3125 
14. Baglio SR, Lagerweij T, Pérez-Lanzón M, Ho XD, Léveillé N, Melo SA, et al. Blocking Tumor-Educated MSC Paracrine Activity Halts Osteosarcoma Progression. Clin Cancer Res. 2017; 23:3721-3733. doi: 10.1158/1078-0432.CCR-16-2726

15. Peinado H, Alečković M, Lavotshkin S, Matei I, Costa-Silva B, Moreno-Bueno G, et al. Melanoma exosomes educate bone marrow progenitor cells toward a pro-metastatic phenotype through MET. Nat Med. 2012, 18:883-91. doi: 10.1038/nm.2753

16. Qiao L, Xu Z, Zhao T, et al. Suppression of tumorigenesis by human mesenchymal stem cells in a hepatoma model. Cell Res. 2008, 18(4):500-507. doi: 10.1038/cr.2008.40

17. Qian BZ, Pollard JW. Macrophage diversity enhances tumor progression and metastasis. Cell. 2010;141(1):39-51. doi: 10.1016/j.cell.2010.03.014

18. Gabrilovich DI, Ostrand-Rosenberg S, Bronte V. Coordinated regulation of myeloid cells by tumours. Nat Rev Immunol. 2012;12(4):253-268. doi: 10.1038/nri3175

19. Wang N, Liang H, Zen K. Molecular mechanisms that influence the macrophage m1-m2 polarization balance. Front Immunol. 2014;5:614. doi: 10.3389/fimmu.2014.00614

20. Chávez-Galán L, Olleros ML, Vesin D, Garcia I. Much More than M1 and M2 Macrophages, There are also $\mathrm{CD} 169(+)$ and $\mathrm{TCR}(+)$ Macrophages. Front Immunol. 2015;6:263. doi: 10.3389/fimmu.2015.00263

21. Biswas SK, Mantovani A. Macrophage plasticity and interaction with lymphocyte subsets: cancer as a paradigm. Nat Immunol. 2010;11(10):889-896. doi: 10.1038/ni.1937

22. Genard G, Lucas S, Michiels C. Reprogramming of Tumor-Associated Macrophages with Anticancer Therapies: Radiotherapy versus Chemo- and Immunotherapies. Front Immunol. 2017;8:828. doi: 10.3389/fimmu.2017.00828

23. Ohri CM, Shikotra A, Green RH, Waller DA, Bradding P. Macrophages within NSCLC tumour islets are predominantly of a cytotoxic M1 phenotype associated with extended survival. Eur Respir J. 2009;33(1):118-126. doi: 10.1183/09031936.00065708

24. Quatromoni JG, Eruslanov E. Tumor-associated macrophages: function, phenotype, and link to prognosis in human lung cancer. Am J Transl Res. 2012;4(4):376-389. PMCID: PMC3493031

25. Hamilton JA. Colony-stimulating factors in inflammation and autoimmunity. Nat Rev Immunol. 2008;8(7):533-544. doi: 10.1038/nri2356

26. Mosser DM, Edwards JP. Exploring the full spectrum of macrophage activation. Nat Rev Immunol. 2008;8(12):958-969. doi: 10.1038/nri2448

27. Chen $\mathrm{P}$, Huang $\mathrm{Y}$, Bong R, et al. Tumor-associated macrophages promote angiogenesis and melanoma growth via adrenomedullin in a paracrine and autocrine manner. Clin Cancer Res. 2011;17(23):7230-7239. doi: 10.1158/1078-0432.CCR-11-1354 
28. Pollard JW. Trophic macrophages in development and disease. Nat Rev Immunol. 2009;9(4):259-270. doi: 10.1038/nri2528

29. Mantovani A, Sica A, Allavena P, Garlanda C, Locati M. Tumor-associated macrophages and the related myeloid-derived suppressor cells as a paradigm of the diversity of macrophage activation. Hum Immunol. 2009;70(5):325-330. doi: 10.1016/j.humimm.2009.02.008

30. Laoui D, Van Overmeire E, Movahedi K, et al. Mononuclear phagocyte heterogeneity in cancer: Different subsets and activation states reaching out at the tumor site. Immunobiology 2011; 216: 1192-1202. doi:10.1016/j.imbio.2011.06.007

31. Wang C, Hu Z, Zhu Z, et al. The MSHA strain of Pseudomonas aeruginosa (PA-MSHA) inhibits gastric carcinoma progression by inducing M1 macrophage polarization. Tumour Biol. 2016;37(5):6913-6921. doi: 10.1007/s13277-015-4451-6

32. Liu J, Duan X. PA-MSHA induces apoptosis and suppresses metastasis by tumor associated macrophages in bladder cancer cells. Cancer Cell Int 2017;17(76) doi 10.1186/ s12935-017-0445-3

33. Wiemann B, Starnes CO. Coley's toxins, tumor necrosis factor and cancer research: a historical perspective. Pharmacol Ther. 1994;64(3):529-564. doi: $\underline{10.1016 / 0163-}$ 7258(94)90023-x

34. Maletzki C, Klier U, Obst W, Kreikemeyer B, Linnebacher M. Reevaluating the concept of treating experimental tumors with a mixed bacterial vaccine: Coley's Toxin. Clin Dev Immunol. 2012;2012:230625. doi: 10.1155/2012/230625

35. Nauts HC, Swift WE, Coley BL. The treatment of malignant tumors by bacerial toxins as developed by the late William B Coley, M.D., reviewed in the light of modern research. Cancer Res. 1946;6:205-216. DOI: Published April 1946

36. Richardson MA, Ramirez T, Russell NC, Moye LA. Coley toxins immunotherapy: a retrospective review. Altern Ther Health Med. 1999;5(3):42-47..

37. Hoption Cann SA, van Netten JP, van Netten C. Dr William Coley and tumour regression: a place in history or in the future. Postgrad Med J. 2003;79:672-680. PMCID: PMC1742910

38. Zacharski LR, Sukhatme VP. Coley's toxin revisited: immunotherapy or plasminogen activator therapy of cancer?. J Thromb Haemost. 2005;3(3):424-427. doi: 10.1111/j.15387836.2005.01110.x

39. McCarthy EF. The toxins of William B. Coley and the treatment of bone and soft-tissue sarcomas. Iowa Orthop J. 2006;26:154-158. PMCID: PMC1888599

40. Krone B, Kölmel KF, Henz BM, Grange JM. Protection against melanoma by vaccination with Bacille Calmette-Guerin (BCG) and/or vaccinia: an epidemiology-based hypothesis on the nature of a melanoma risk factor and its immunological control. Eur J Cancer. 2005;41(1):104-117. doi: 10.1016/j.ejca.2004.08.010 
41. Sakuishi K, Jayaraman P, Behar SM, Anderson AC, Kuchroo VK. Emerging Tim-3 functions in antimicrobial and tumor immunity. Trends Immunol. 2011;32(8):345-349. doi: 10.1016/j.it.2011.05.003

42. Mantovani A, Sozzani S, Locati M, Allavena P, Sica A. Macrophage polarization: tumorassociated macrophages as a paradigm for polarized M2 mononuclear phagocytes. Trends Immunol. 2002;23(11):549-555. doi: 10.1016/s1471-4906(02)02302-5

43. Mantovani A, Sica A, Locati M. Macrophage polarization comes of age. Immunity. 2005;23(4):344-346. doi: 10.1016/j.immuni.2005.10.001

44. Sica A, Larghi P, Mancino A, et al. Macrophage polarization in tumour progression. Semin Cancer Biol. 2008;18(5):349-355. doi: 10.1016/j.semcancer.2008.03.004

45. Paget $\mathrm{S}$. The distribution of secondary growths in cancer of the breast. Lancet 1889;1:571573. doi: 10.1016/S0140-6736(00)49915-0

46. Psaila B, Lyden D. The metastatic niche: adapting the foreign soil. Nat Rev Cancer. 2009;9(4):285-293. doi: 10.1038/nrc2621

47. Kaplan RN, Riba RD, Zacharoulis S, et al. VEGFR1-positive haematopoietic bone marrow progenitors initiate the pre-metastatic niche. Nature. 2005;438(7069):820-827. doi: 10.1038 /nature04186

48. Chin AR, Wang SE. Cancer Tills the Premetastatic Field: Mechanistic Basis and Clinical Implications. Clin Cancer Res. 2016;22(15):3725-3733. doi: 10.1158/1078-0432.CCR-160028

49. Géraud C, Koch PS, Damm F, Schledzewski K, Goerdt S. The metastatic cycle: metastatic niches and cancer cell dissemination. J Dtsch Dermatol Ges. 2014;12(11):1012-1019. doi: $10.1111 /$ ddg.12451

50. Joyce JA, Pollard JW. Microenvironmental regulation of metastasis. Nat Rev Cancer. 2009;9(4):239-252. doi: 10.1038/nrc2618

51. Yáñez-Mó M, Siljander PR, Andreu Z, et al. Biological properties of extracellular vesicles and their physiological functions. J Extracell Vesicles. 2015;4:27066. Published 2015 May 14. doi: $10.3402 /$ jev.v4.27066

52. Théry C, Witwer KW, Aikawa E, et al. Minimal information for studies of extracellular vesicles 2018 (MISEV2018): a position statement of the International Society for Extracellular Vesicles and update of the MISEV2014 guidelines. J Extracell Vesicles. 2018;7(1):1535750. Published 2018 Nov 23. doi: 10.1080/20013078.2018.1535750

53. Wu M, Wang G, Hu W, Yao Y, Yu XF. Emerging roles and therapeutic value of exosomes in cancer metastasis. Mol Cancer. 2019;18(1):53. doi: 10.1186/s12943-019-0964-8 
54. Harmati M, Gyukity-Sebestyen E, Dobra G, et al. Small extracellular vesicles convey the stress-induced adaptive responses of melanoma cells. Sci Rep. 2019;9(1):15329. doi: 10.1038/s41598-019-51778-6

55. Chaffer CL, Weinberg RA. A perspective on cancer cell metastasis. Science 2011;331:155964. doi: $10.1126 /$ science. 1203543

56. Peinado, H., Zhang, H., Matei, I. et al. Pre-metastatic niches: organ-specific homes for metastases. Nat Rev Cancer 2017;17,302-317 doi: 10.1038/nrc.2017.6

57. Meehan B, Rak J, Di Vizio D. Oncosomes - large and small: what are they, where they came from?. J Extracell Vesicles. 2016;5:33109. doi: 10.3402/jev.v5.33109

58. Chen W, Hoffmann AD, Liu H, Liu X. Organotropism: new insights into molecular mechanisms of breast cancer metastasis. NPJ Precis Oncol. 2018;2(1):4. doi: 10.1038/ s41698-018-0047-0

59. Ekstrom EJ, Bergenfelz C, von Bulow V, et al. WNT5A induces release of exosomes containing pro-angiogenic and immunosuppressive factors from malignant melanoma cells. Mol Cancer. 2014;13:88 doi: 10.1186/1476-4598-13-88

60. McAllister SS, Weinberg RA. The tumour-induced systemic environment as a critical regulator of cancer progression and metastasis. Nat Cell Biol. 2014;16(8):717-727. doi: $10.1038 / \mathrm{ncb} 3015$

61. Roma-Rodrigues C, Fernandes AR, Baptista PV. Exosome in tumour microenvironment: overview of the crosstalk between normal and cancer cells. Biomed Res Int. 2014;2014:179486. doi: 10.1155/2014/179486

62. International Agency for Research on Cancer, WHO. GLOBOCAN 2020: estimated cancer incidence, mortality, and prevalence worldwide in 2018. http://globocan.iarc.fr (accessed Feb 05, 2020).

63. Miller AJ, Mihm MC Jr. Melanoma. N Engl J Med. 2006;355(1):51-65. doi: 10.1056/ NEJMra052166

64. Shain AH, Yeh I, Kovalyshyn I, et al. The genetic evolution of melanoma from precursor lesions. N Engl J Med 2015;373: 1926-36 doi: 10.1056/NEJMoa1502583

65. Hodis E, Watson IR, Kryukov GV, et al. A landscape of driver mutations in melanoma. Cell. 2012;150(2):251-263. doi: 10.1016/j.cell.2012.06.024

66. Cancer Genome Atlas Network. Genomic Classification of Cutaneous Melanoma. Cell. 2015;161(7):1681-1696. doi: 10.1016/j.cell.2015.05.044

67. Jakob JA, Bassett RL Jr, Ng CS, et al. NRAS mutation status is an independent prognostic factor in metastatic melanoma. Cancer. 2012;118(16):4014-4023. doi: 10.1002/cncr.26724

68. Umansky V, Sevko A. Melanoma-induced immunosuppression and its neutralization. Semin Cancer Biol. 2012;22(4):319-326. doi: 10.1016/j.semcancer.2012.02.003 
69. Garcia-Diaz A, Shin DS, Moreno BH, et al. Interferon Receptor Signaling Pathways Regulating PD-L1 and PD-L2 Expression. Cell Rep. 2017;19(6):1189-1201. doi: 10.1016/j. celrep.2017.04.031

70. Kleffel S, Posch C, Barthel SR, et al. Melanoma Cell-Intrinsic PD-1 Receptor Functions Promote Tumor Growth. Cell. 2015;162(6):1242-1256. doi: 10.1016/j.cell.2015.08.052

71. Pieniazek M, Matkowski R, Donizy P. Macrophages in skin melanoma-the key element in melanomagenesis. Oncol Lett. 2018;15(4):5399-5404. doi: 10.3892/ol.2018.8021

72. Szebeni GJ, Kriston-Pál É, Blazsó P, et al. Identification of galectin-1 as a critical factor in function of mouse mesenchymal stromal cell-mediated tumor promotion. PLoS One. 2012;7(7):e41372. doi: 10.1371/journal.pone.0041372

73. Campbell LA, Kuo CC. Cultivation and laboratory maintenance of Chlamydia pneumoniae. Curr Protoc Microbiol. 2009; Chapter 11:Unit11B.1. doi: 10.1002/9780471729259. mc11b01s12

74. Carpenter AE, Jones TR, Lamprecht MR, et al. CellProfiler: image analysis software for identifying and quantifying cell phenotypes. Genome Biol. 2006;7(10):R100. doi: 10.1186/ gb-2006-7-10-r100

75. Olivo-Marin JC. Extraction of spots in biological images using multiscale products. Pattern Recognit. 2002;35(9):1989-1996. doi: 10.1016/S0031-3203(01)00127-3

76. van de Linde S, Löschberger A, Klein T, et al. Direct stochastic optical reconstruction microscopy with standard fluorescent probes. Nat Protoc. 2011;6(7):991-1009. doi: 10.1038/ nprot.2011.336

77. Rees EJ, Erdelyi M, Kaminski-Schierle GS, Knight AE, Kaminski CF. Elements of image processing in localisation microscopy. J Opt 2013;15:094012. doi:10.1088/2040$\underline{8978 / 15 / 9 / 094012}$

78. Donizy P, Zietek M, Halon A, Leskiewicz M, Kozyra C, Matkowski R. Prognostic significance of ALCAM (CD166/MEMD) expression in cutaneous melanoma patients. Diagn Pathol. 2015;10:86. Published 2015 Jul 2. doi: 10.1186/s13000-015-0331-z

79. Ferretti R, Bhutkar A, McNamara MC, Lees JA. BMI1 induces an invasive signature in melanoma that promotes metastasis and chemoresistance. Genes Dev. 2016;30(1):18-33. doi: $10.1101 / \mathrm{gad} .267757 .115$

80. Mummert ME, Mummert DI, Ellinger L, Takashima A. Functional roles of hyaluronan in B16-F10 melanoma growth and experimental metastasis in mice. Mol Cancer Ther. 2003;2(3):295-300.

81. Muñoz R, Arias Y, Ferreras JM, et al. In vitro and in vivo effects of an anti-mouse endoglin (CD105)-immunotoxin on the early stages of mouse B16MEL4A5 melanoma 
tumours. Cancer Immunol Immunother. 2013;62(3):541-551. doi: 10.1007/s00262-012$\underline{1357-7}$

82. Hazarika P, McCarty MF, Prieto VG, et al. Up-regulation of Flotillin-2 is associated with melanoma progression and modulates expression of the thrombin receptor protease activated receptor 1. Cancer Res. 2004;64(20):7361-7369. doi: 10.1158/0008-5472.CAN04-0823

83. Lee N, Barthel SR, Schatton T. Melanoma stem cells and metastasis: mimicking hematopoietic cell trafficking?. Lab Invest. 2014;94(1):13-30. doi: 10.1038/labinvest.2013.116

84. Ahmed KBR, Davies AM. New molecular targets for the systemic therapy of melanoma. In: Murph M, editor. Research on Melanoma - A Glimpse into Current Directions and Future Trends. London, UK: Intech 2011. p. 161-80.

85. Dunleavey JM, Xiao L, Thompson J, et al. Vascular channels formed by subpopulations of PECAM1+ melanoma cells. Nat Commun. 2014;5:5200. Published 2014 Oct 22. doi: $10.1038 /$ ncomms 6200

86. Madjd Z, Erfani E, Gheytanchi E, Moradi-Lakeh M, Shariftabrizi A, Asadi-Lari M. Expression of CD133 cancer stem cell marker in melanoma: a systematic review and meta-analysis. Int J Biol Markers. 2016;31(2):e118-e125. doi: 10.5301/jbm.5000209

87. Schubert K, Gutknecht D, Köberle M, Anderegg U, Saalbach A. Melanoma cells use Thy1 (CD90) on endothelial cells for metastasis formation. Am J Pathol. 2013;182(1):266-276. doi: 10.1016/j.ajpath.2012.10.003

88. Stengel K, Zheng Y. Cdc42 in oncogenic transformation, invasion, and tumorigenesis. Cell Signal. 2011;23(9):1415-1423. doi: 10.1016/j.cellsig.2011.04.001

89. Minard ME, Kim LS, Price JE, Gallick GE. The role of the guanine nucleotide exchange factor Tiam1 in cellular migration, invasion, adhesion and tumor progression. Breast Cancer Res Treat. 2004;84(1):21-32. doi: 10.1023/B:BREA.0000018421.31632.e6

90. Raisova M, Hossini AM, Eberle J, et al. The Bax/Bcl-2 ratio determines the susceptibility of human melanoma cells to CD95/Fas-mediated apoptosis. $J$ Invest Dermatol. 2001;117(2):333-340. doi: 10.1046/j.0022-202x.2001.01409.x

91. Stupack DG. Caspase-8 as a therapeutic target in cancer. Cancer Lett. 2013;332(2):133140. doi: $10.1016 /$ j.canlet.2010.07.022

92. Chen J, Zhang XD. Nanodelivery of anticancer agents in melanoma: encouraging, but a long way to go. In: Hamblin M, Avci P, Prow T, editors. Nanoscience in Dermatology. Cambridge: Academic Press 2016. p. 189-202. doi:10.1016/B978-0-12-802926-8.00015-X

93. Hügel R, Muendlein A, Volbeding L, et al. Serum levels of hepatocyte growth factor as a potential tumor marker in patients with malignant melanoma. Melanoma Res. 2016;26(4):354-360. doi: 10.1097/CMR.0000000000000269 
94. Nicholas C, Lesinski GB. The Jak-STAT signal transduction pathway in melanoma. In: Tanaka Y, editor. Breakthroughs in Melanoma Research. London, UK: IntechOpen. 2011. p. 283-306. doi:10.5772/18876

95. Al-U'datt DGF, Al-Husein BAA, Qasaimeh GR. A mini-review of c-Met as a potential therapeutic target in melanoma. Biomed Pharmacother. 2017;88:194-202. doi: 10.1016/j. biopha.2017.01.045

96. Ramsay RG, Gonda TJ. MYB function in normal and cancer cells. Nat Rev Cancer. 2008;8(7):523-534. doi: 10.1038/nrc2439

97. Cao HH, Chu JH, Kwan HY, et al. Inhibition of the STAT3 signaling pathway contributes to apigenin-mediated anti-metastatic effect in melanoma. Sci Rep. 2016;6:21731. doi: 10.1038/ srep21731

98. Roesch A, Becker B, Meyer S, et al. Overexpression and hyperphosphorylation of retinoblastoma protein in the progression of malignant melanoma. Mod Pathol. 2005;18(4):565-572. doi: 10.1038/modpathol.3800324

99. Kyriakis JM, App H, Zhang XF, et al. Raf-1 activates MAP kinase-kinase. Nature. 1992;358(6385):417-421. doi: 10.1038/358417a0

100. Li A, Machesky LM. Rac1 cycling fast in melanoma with P29S [published online ahead of print, 2013 Feb 8]. Pigment Cell Melanoma Res. 2013;10.1111/pcmr.12074. doi: 10.1111/ pcmr.12074

101. Flaherty KT, Hodi FS, Fisher DE. From genes to drugs: targeted strategies for melanoma. Nat Rev Cancer. 2012;12(5):349-361. doi: 10.1038/nrc3218

102. Mantovani A, Biswas SK, Galdiero MR, Sica A, Locati M. Macrophage plasticity and polarization in tissue repair and remodelling. J Pathol. 2013;229(2):176-185. doi: 10.1002/ path.4133

103. Sica A, Mantovani A. Macrophage plasticity and polarization: in vivo veritas. $J$ Clin Invest. 2012;122(3):787-795. doi: 10.1172/JCI59643

104. Balkwill F. Tumour necrosis factor and cancer. Nat Rev Cancer. 2009;9(5):361-371. doi: $10.1038 / \mathrm{nrc} 2628$

105. Schepsky A, Bruser K, Gunnarsson GJ, et al. The microphthalmia-associated transcription factor Mitf interacts with beta-catenin to determine target gene expression. Mol Cell Biol. 2006;26(23):8914-8927. doi: 10.1128/MCB.02299-05

106. Chen J, Shao R, Zhang XD, Chen C. Applications of nanotechnology for melanoma treatment, diagnosis, and theranostics. Int J Nanomedicine. 2013;8:2677-2688. doi: 10.2147/ IJN.S45429 
107. Iida N, Dzutsev A, Stewart CA, et al. Commensal bacteria control cancer response to therapy by modulating the tumor microenvironment. Science. 2013;342(6161):967-970. doi: $10.1126 /$ science. 1240527

108. Hobohm U. Fever and cancer in perspective. Cancer Immunol Immunother. 2001;50(8):391396. doi: $10.1007 / \mathrm{s} 002620100216$

109. Prebeck S, Kirschning C, Dürr S, et al. Predominant role of toll-like receptor 2 versus 4 in Chlamydia pneumoniae-induced activation of dendritic cells. J Immunol. (2001) 167(6):3316-3323. doi: 10.4049/jimmunol.167.6.3316 


\section{SUPPLEMENTARY MATERIAL}

a

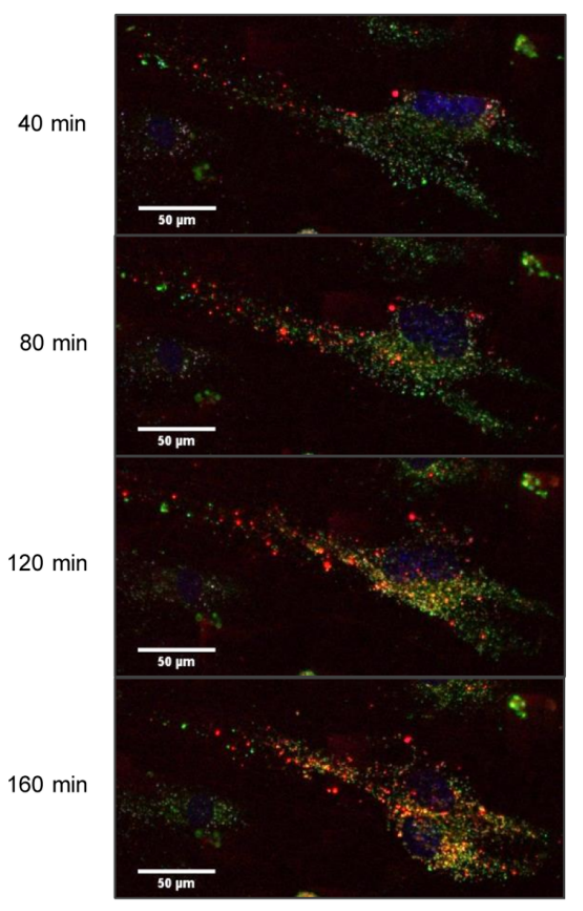

b

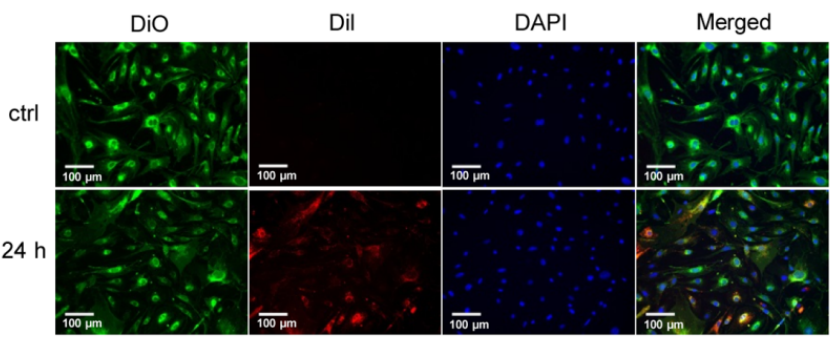

Figure S1. Kinetics of exosome uptake by MSCs.

(a) Fluorescent images of exosome uptake by MSCs in early timepoints. DiO (green fluorescent lipid dye) and Hoechst 33342 (nucleic acid stain)-labeled cells were exposed to Dil (red fluorescent lipid dye)-labeled exosomes and were investigated by a Celldiscoverer 7 automated live cell imaging system (Zeiss). Internalization of exosomes was visible as early as $40 \mathrm{~min}$ after exosome exposure. (b) Fluorescent images of exosome uptake by MSCs $24 \mathrm{~h}$ after exosome exposure. Images were taken by an Operetta high content screening system. The amount of internalized exosomes showed cell to cell variation. 


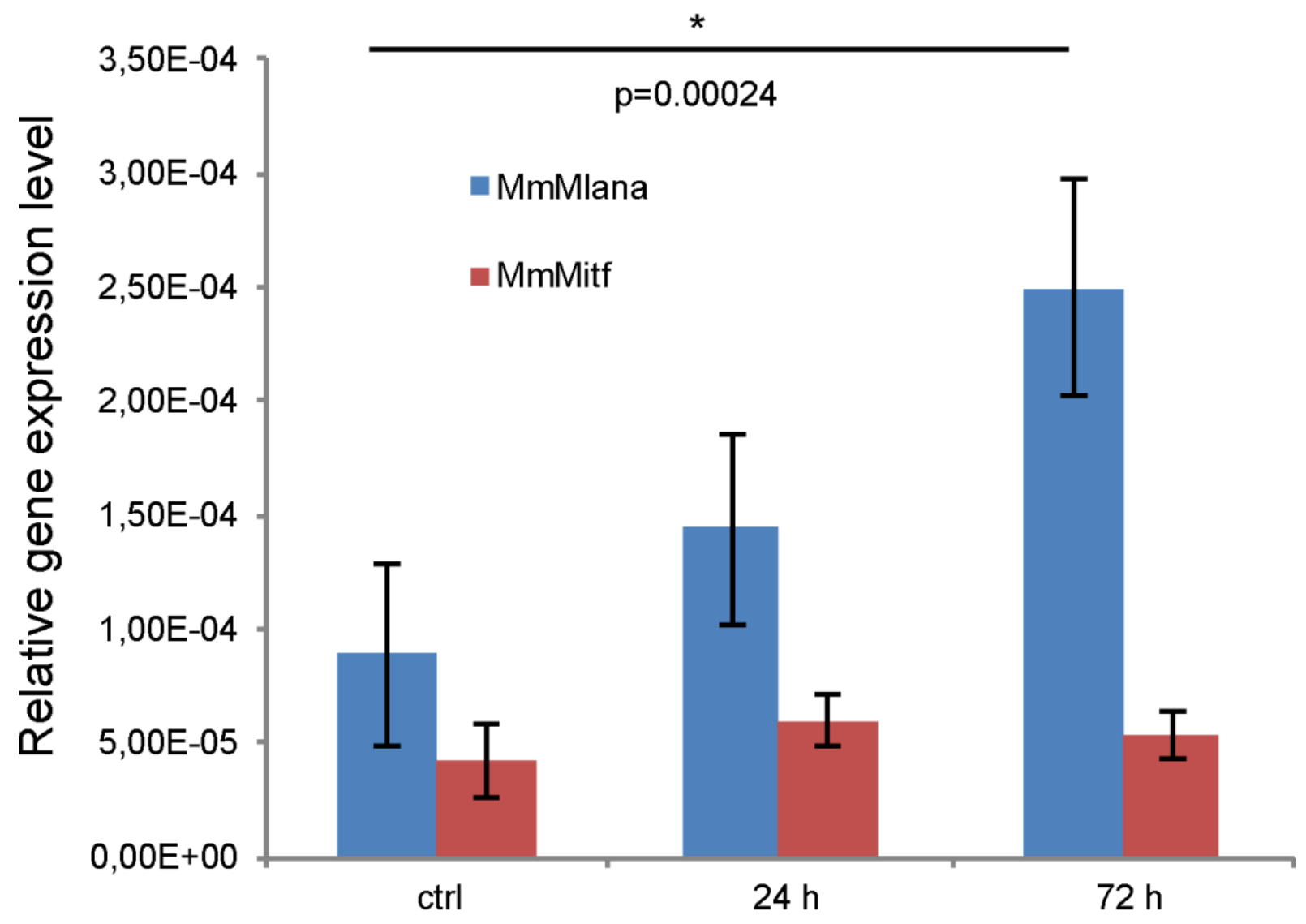

Figure S2. QRT-PCR analysis of Mlana and Mitf in exosome-exposed MSCs.

The Mlana and Mitf mRNA levels were determinated by QRT-PCR using a SyberGreen protocol after $24 \mathrm{~h}$ and $72 \mathrm{~h}$ of exosome exposure. Results are presented as mean $\pm S D(\mathrm{n}=3)$. 


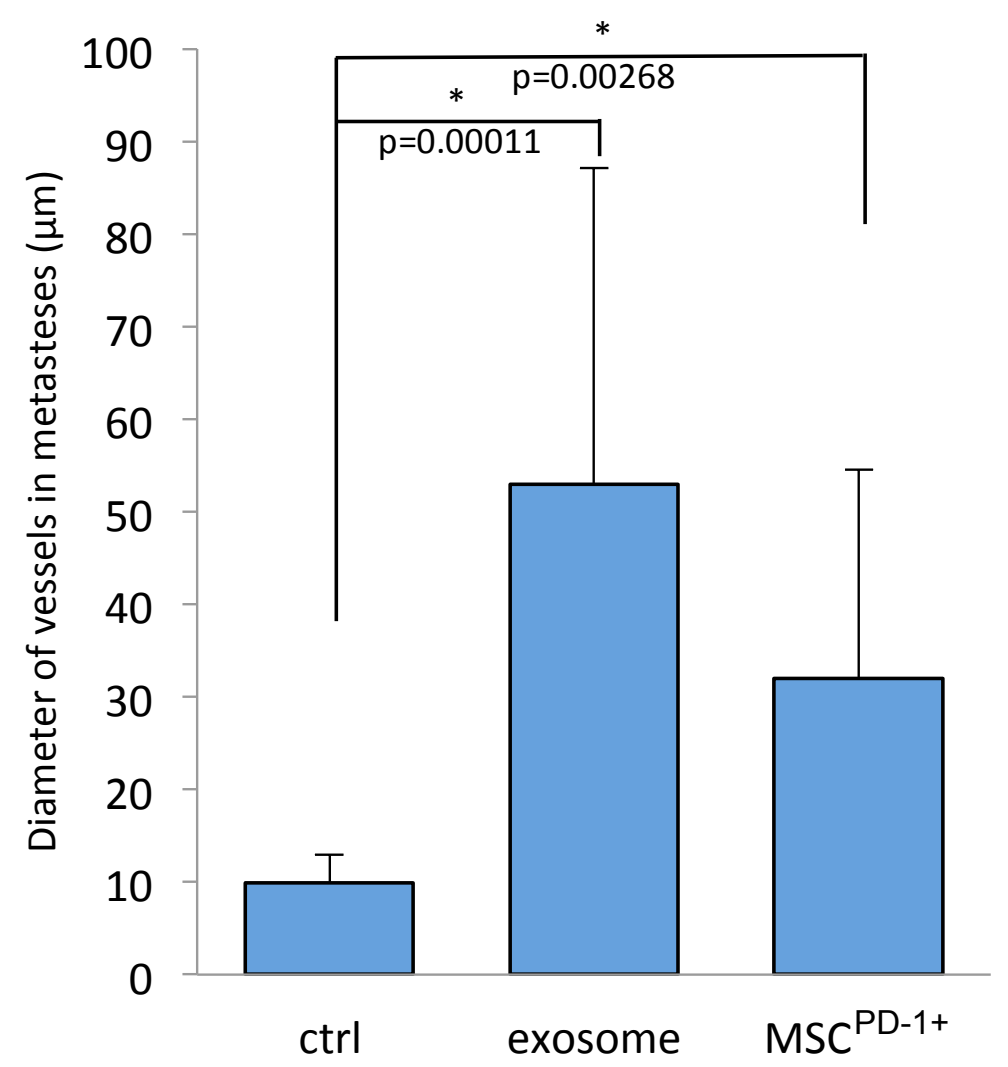

Figure S3. Quantification of vessel diameter of tumor-associated blood vessels in melanoma metastases. Diameter of vessels significantly increased in the metastases of exosome and $\mathrm{MSC}^{\mathrm{PD} 1+}$ injected groups of tumorbearing mice inside the lung metastases. 
a

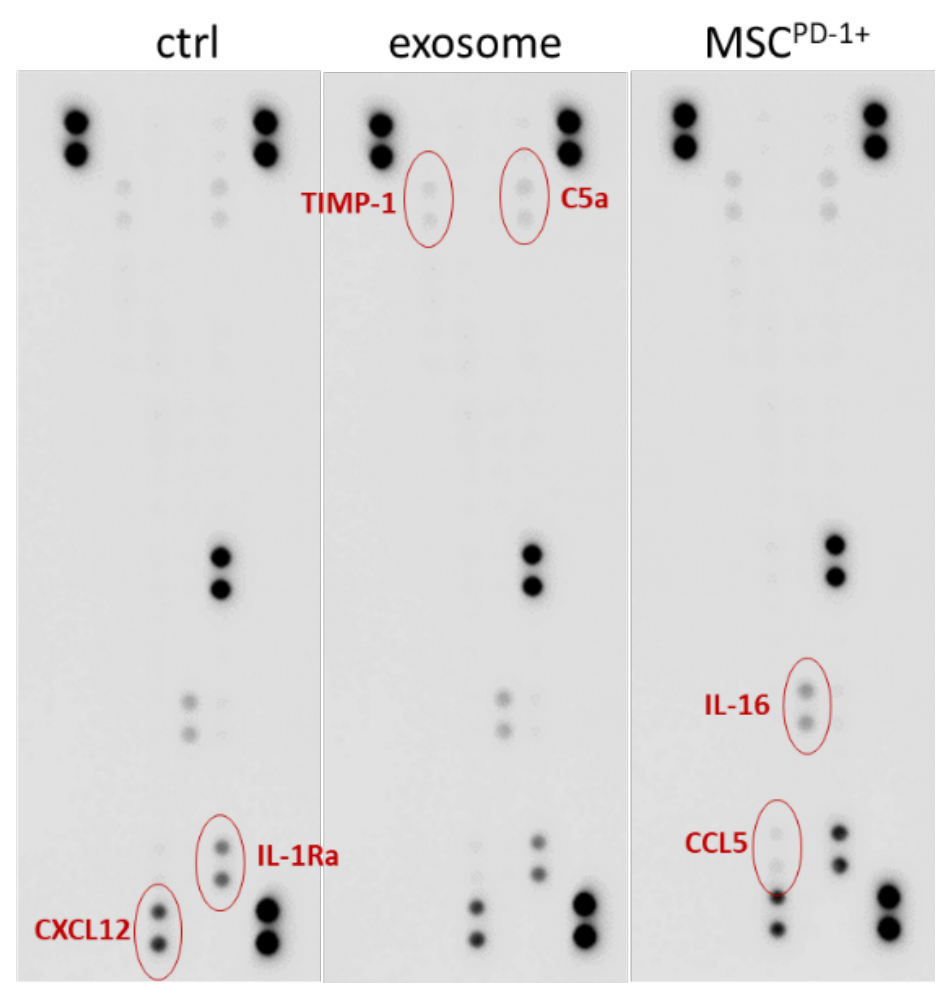

b

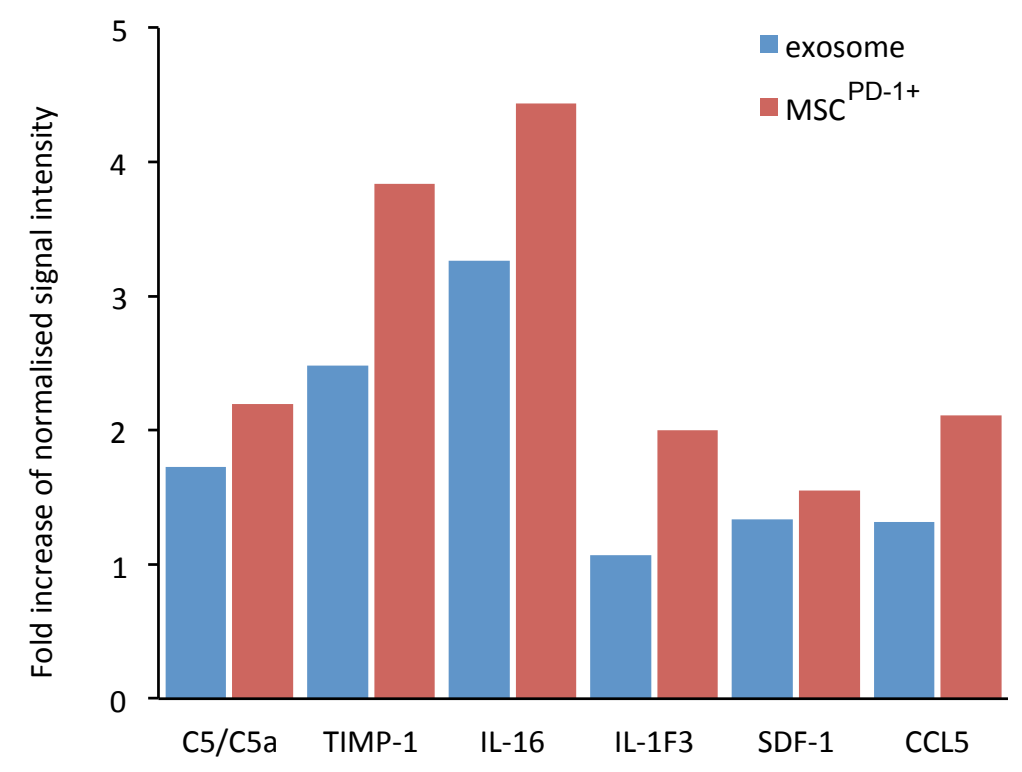

Figure S4. Exosomes and $\mathrm{MSC}^{\mathrm{PD}-1+}$ administration-induced cytokine profiles in the lung tissues of tumorbearing mice. (a) cytokine array panels of the lung tissue lysates, cytokines with elevated levels are marked. (b) bar graph shows the fold increase values of cytokines for both groups compared to the ctrl one. Data were obtained by densitometry. 
Table S1. Treatment schedule of animals

\begin{tabular}{ccccc}
\hline Group & B16F1 & Control buffer & MSC $^{\mathrm{PD}-1+}$ i.v. & B16F1 exosome i.v. \\
\hline ctrl & + & + & - & - \\
MSC $^{\mathrm{PD}-1+}$ & + & - & + & - \\
exosome & + & - & - & + \\
\hline
\end{tabular}

Table S2. Mlana and Mitf primers used in QRT-PCR

\begin{tabular}{lcccc}
\hline Gene name & Symbol & Ref. seq. ID & $\begin{array}{c}\text { Forward primer } \\
\text { sequence }\end{array}$ & $\begin{array}{c}\text { Reverse primer } \\
\text { sequence }\end{array}$ \\
\hline $\begin{array}{l}\text { melan-A } \\
\text { microphthalmia-associated }\end{array}$ & Mlana & NM_029993.1 & ggtcctggggattgctct & caatatgacgecttttgtcca \\
\begin{tabular}{l} 
transcription factor \\
\hline
\end{tabular} & NM_008601.3 & ctaagtggtctgcggtgtctc & ggttttccaggtgggtctg \\
\hline
\end{tabular}

Primers were designed using the online Roche Universal Probe Library Assay Design Center. The quality of the primers was verified by MS analysis provided by Bioneer (Daejeon, Korea). 
Table S3. List of exosomal proteins identified by LC-MSMS

\begin{tabular}{|c|c|c|}
\hline ID & Symbol & Entrez Gene Name \\
\hline P97857 & ADAMTS1 & ADAM metallopeptidase with thrombospondin type 1 motif 1 \\
\hline Q3TNX8 & ADAMTS4 & ADAM metallopeptidase with thrombospondin type 1 motif 4 \\
\hline Q640N1 & AEBP1 & AE binding protein 1 \\
\hline P05064 & ALDOA & aldolase, fructose-bisphosphate A \\
\hline P97429 & ANXA4 & annexin A4 \\
\hline P48036 & ANXA5 & annexin A5 \\
\hline P08226 & APOE & apolipoprotein E \\
\hline Q3TWT5 & ASAH1 & $\mathrm{N}$-acylsphingosine amidohydrolase 1 \\
\hline Q3TXF9 & ATP1A1 & ATPase $\mathrm{Na}+/ \mathrm{K}+$ transporting subunit alpha 1 \\
\hline P97370 & ATP1B3 & ATPase $\mathrm{Na}+\mathrm{K}+$ transporting subunit beta 3 \\
\hline Q1XID4 & ATP6AP2 & ATPase $\mathrm{H}+$ transporting accessory protein 2 \\
\hline Q9JL18 & BACE2 & beta-site APP-cleaving enzyme 2 \\
\hline O55107 & BSG & basigin (Ok blood group) \\
\hline Q8R2Q8 & Bst2 & bone marrow stromal cell antigen 2 \\
\hline Q9WVT6 & CA14 & carbonic anhydrase 14 \\
\hline P41731 & CD63 & CD63 molecule \\
\hline P35762 & CD81 & CD81 molecule \\
\hline P10605 & CTSB & cathepsin B \\
\hline P18242 & CTSD & cathepsin D \\
\hline P29812 & DCT & dopachrome tautomerase \\
\hline P57776 & EEF1D & eukaryotic translation elongation factor 1 delta \\
\hline Q3UAM9 & ENG & endoglin \\
\hline P17182 & ENO1 & enolase 1 \\
\hline P19096 & FASN & fatty acid synthase \\
\hline P30416 & FKBP4 & FK506 binding protein 4 \\
\hline P11276 & FN1 & fibronectin 1 \\
\hline P09528 & FTH1 & ferritin heavy chain 1 \\
\hline P16858 & GAPDH & glyceraldehyde-3-phosphate dehydrogenase \\
\hline P08752 & GNAI2 & $\mathrm{G}$ protein subunit alpha i2 \\
\hline Q3TAV1 & GPNMB & glycoprotein nmb \\
\hline P19157 & GSTP1 & glutathione S-transferase pi 1 \\
\hline P11499 & HSP90AB1 & heat shock protein 90 alpha family class B member 1 \\
\hline B1B0C7 & HSPG2 & heparan sulfate proteoglycan 2 \\
\hline Q9CQW9 & IFITM3 & interferon induced transmembrane protein 3 \\
\hline G3UYZ1 & IGSF8 & immunoglobulin superfamily member 8 \\
\hline Q91VK4 & ITM2C & integral membrane protein $2 \mathrm{C}$ \\
\hline P02468 & LAMC1 & laminin subunit gamma 1 \\
\hline Q60961 & LAPTM4A & lysosomal protein transmembrane 4 alpha \\
\hline P35951 & LDLR & low density lipoprotein receptor \\
\hline Q07797 & LGALS3BP & galectin 3 binding protein \\
\hline Q3U2W5 & LGALS8 & galectin 8 \\
\hline P16056 & MET & MET proto-oncogene, receptor tyrosine kinase \\
\hline P21956 & MFGE8 & milk fat globule-EGF factor 8 protein \\
\hline Q2TA50 & MLANA & melan-A \\
\hline Q6NVG5 & MREG & melanoregulin \\
\hline Q9EPX2 & PAPLN & papilin, proteoglycan like sulfated glycoprotein \\
\hline Q3UIP2 & PCOLCE & procollagen C-endopeptidase enhancer \\
\hline Q811J2 & LOC72520 & LOC72520 protein \\
\hline
\end{tabular}




\begin{tabular}{|c|c|c|}
\hline $\begin{array}{l}\text { Table S3. } \\
\text { ID }\end{array}$ & Symbol & Entrez Gene Name \\
\hline Q80Y09 & PDCD6IP & programmed cell death 6 interacting protein \\
\hline P62962 & PFN1 & profilin 1 \\
\hline P09411 & PGK1 & phosphoglycerate kinase 1 \\
\hline P52480 & PKM & pyruvate kinase, muscle \\
\hline Q9CZB2 & PMEL & premelanosome protein \\
\hline P17742 & PPIA & peptidylprolyl isomerase A \\
\hline P35700 & PRDX1 & peroxiredoxin 1 \\
\hline Q61171 & PRDX2 & peroxiredoxin 2 \\
\hline Q543S0 & PRELP & proline and arginine rich end leucine rich repeat protein \\
\hline P53994 & RAB2A & RAB2A, member RAS oncogene family \\
\hline Q8CCG5 & RALB & RAS like proto-oncogene B \\
\hline O89086 & RBM3 & RNA binding motif (RNP1, RRM) protein 3 \\
\hline P35980 & RPL18 & ribosomal protein L18 \\
\hline Q3U5P4 & SCPEP1 & serine carboxypeptidase 1 \\
\hline O08992 & SDCBP & syndecan binding protein \\
\hline Q0VGP2 & SEMA3B & semaphorin 3B \\
\hline P32261 & SERPINC1 & serpin family $\mathrm{C}$ member 1 \\
\hline P10852 & SLC3A2 & solute carrier family 3 member 2 \\
\hline Q3UQM7 & SLC7A5 & solute carrier family 7 member 5 \\
\hline O09044 & SNAP23 & synaptosome associated protein 23 \\
\hline Q64337 & SQSTM1 & sequestosome 1 \\
\hline Q8CI59 & STEAP3 & STEAP3 metalloreductase \\
\hline Q3TDG9 & STX12 & syntaxin 12 \\
\hline O70439 & STX7 & syntaxin 7 \\
\hline P40749 & SYT4 & synaptotagmin 4 \\
\hline O88968 & TCN2 & transcobalamin 2 \\
\hline Q542D9 & TFRC & transferrin receptor \\
\hline P39876 & TIMP3 & TIMP metallopeptidase inhibitor 3 \\
\hline Q4FJX7 & TINAGL1 & tubulointerstitial nephritis antigen like 1 \\
\hline Q9DCS1 & TMEM176A & transmembrane protein $176 \mathrm{~A}$ \\
\hline Q9R1Q6 & TMEM176B & transmembrane protein $176 \mathrm{~B}$ \\
\hline Q9CZX7 & TMEM55A & transmembrane protein $55 \mathrm{~A}$ \\
\hline Q9QY73 & TMEM59 & transmembrane protein 59 \\
\hline O88746 & TOM1 & target of myb1 membrane trafficking protein \\
\hline O89023 & TPP1 & tripeptidyl peptidase 1 \\
\hline Q3UCW0 & TSG101 & tumor susceptibility 101 \\
\hline Q4FJW7 & TSPAN4 & tetraspanin 4 \\
\hline Q8BJU2 & TSPAN9 & tetraspanin 9 \\
\hline P11344 & TYR & tyrosinase \\
\hline P07147 & TYRP1 & tyrosinase related protein 1 \\
\hline O70404 & VAMP8 & vesicle associated membrane protein 8 \\
\hline Q8R0J7 & VPS37B & VPS37B, ESCRT-I subunit \\
\hline Q8R105 & VPS37C & VPS37C, ESCRT-I subunit \\
\hline O88384 & VTI1B & vesicle transport through interaction with t-SNAREs $1 \mathrm{~B}$ \\
\hline A8DUQ1 & HBBT1 & beta-globin \\
\hline P70356 & MELA & Gag-pol poliprotein \\
\hline P70355 & MELA & envelope protein \\
\hline
\end{tabular}


Table S4. List of exosomal miRNAs identified by SOLiD 5500xl technology

\begin{tabular}{|c|c|c|c|c|c|}
\hline Symbol & Seed regio & ID & Symbol & Seed regio & ID \\
\hline \multirow[t]{4}{*}{ let- $7 a-3 p$} & UAUACAA & mmu-let-7a-1-3p & miR-146a-5p & GAGAACU & mmu-mir-146a-5p \\
\hline & & mmu-let-7b-3p & $\operatorname{miR}-148 a-3 p$ & CAGUGCA & mmu-mir-148b-3p \\
\hline & & mmu-let-7c-2-3p & miR-151-3p & UAGACUG & mmu-mir-151-3p \\
\hline & & mmu-let-7f-1-3p & $\operatorname{miR}-15 a-3 p$ & AGGCCAU & mmu-mir-15a-3p \\
\hline \multirow[t]{7}{*}{ let-7a-5p } & GAGGUAG & mmu-let-7a-5p & & & mmu-mir- $15 b-5 p$ \\
\hline & & mmu-let-7b-5p & & & mmu-mir-16-5p \\
\hline & & mmu-let-7c-5p & & & mmu-mir-195a-5p \\
\hline & & mmu-let-7d-5p & & & mmu-mir-322-5p \\
\hline & & mmu-let-7e-5p & & & mmu-mir-497a-5p \\
\hline & & mmu-let-7f-5p & $\operatorname{miR}-17-3 p$ & CUGCAGU & mmu-mir-17-3p \\
\hline & & mmu-let-7g-5p & miR-17-5p & AAAGUGC & mmu-mir-106b-5p \\
\hline let-7d-3p & UAUACGA & $\begin{array}{l}\text { mmu-mir-98-5p } \\
\text { mmu-let-7d-3p }\end{array}$ & & & $\begin{array}{l}\text { mmu-mir-17-5p } \\
\text { mmu-mir-20a-5p }\end{array}$ \\
\hline let-7i-3p & UGCGCAA & mmu-let-7i-3p & & & mmu-mir-93-5p \\
\hline \multirow[t]{2}{*}{$\operatorname{miR}-100-5 p$} & ACCCGUA & mmu-mir-99a-5p & $\begin{array}{l}\text { miR-181a- } \\
1-3 p\end{array}$ & CCAUCGA & mmu-mir-181a-1-3p \\
\hline & & mmu-mir-99b-5p & miR-181a-5p & ACAUUCA & mmu-mir-181a-5p \\
\hline miR-101-3p & ACAGUAC & mmu-mir-101a-3p & & & mmu-mir-181b-5p \\
\hline miR-103-1-5p & GCUUCUU & mmu-mir-107-5p & & & mmu-mir-181c-5p \\
\hline \multirow[t]{2}{*}{ miR-103-3p } & GCAGCAU & mmu-mir-103-3p & & & mmu-mir-181d-5p \\
\hline & & mmu-mir-107-3p & miR-1827 & GAGGCAG & mmu-mir-709 \\
\hline \multirow[t]{2}{*}{$\operatorname{miR}-10 a-5 p$} & ACCCUGU & mmu-mir-10a-5p & miR-1839-3p & GACCUAC & mmu-mir-1839-3p \\
\hline & & mmu-mir-10b-5p & miR-185-5p & GGAGAGA & mmu-mir-185-5p \\
\hline miR-1191a & AGUCUUA & mmu-mir-1191a & miR-186-5p & AAAGAAU & mmu-mir-186-5p \\
\hline miR-1249-3p & CGCCCUU & mmu-mir-1249-3p & miR-187-3p & CGUGUCU & mmu-mir-187-3p \\
\hline \multirow{3}{*}{$\operatorname{miR}-125 b-5 p$} & CCCUGAG & mmu-mir-125a-5p & $\operatorname{miR}-188-3 p$ & UCCCACA & mmu-mir-188-3p \\
\hline & & mmu-mir- $125 b-5 p$ & miR-188-5p & AUCCCUU & mmu-mir-188-5p \\
\hline & & mmu-mir-351-5p & $\operatorname{miR}-18 a-5 p$ & AAGGUGC & mmu-mir-18a-5p \\
\hline miR-126a-5p & AUUAUUA & mmu-mir-126a-5p & miR-191-5p & AACGGAA & mmu-mir-191-5p \\
\hline $\operatorname{miR}-128-3 p$ & CACAGUG & $\mathrm{mmu}-\mathrm{mir}-128-3 \mathrm{p}$ & miR-193a-3p & ACUGGCC & mmu-mir-193a-3p \\
\hline \multirow[t]{2}{*}{ miR-129-1-3p } & AGCCCUU & mmu-mir-129-1-3p & miR-1981-3p & AUCUAAC & mmu-mir-1981-3p \\
\hline & & mmu-mir-129-2-3p & miR-199a-3p & CAGUAGU & mmu-mir-199a-3p \\
\hline $\operatorname{miR}-129 b-5 p$ & CUUUUUG & mmu-mir-129b-5p & & & mmu-mir-199b-3p \\
\hline \multirow[t]{4}{*}{ miR-130a-3p } & AGUGCAA & mmu-mir-130a-3p & miR-199a-5p & CCAGUGU & mmu-mir-199a-5p \\
\hline & & mmu-mir-130b-3p & & & mmu-mir-199b-5p \\
\hline & & mmu-mir-301a-3p & miR-19b-3p & GUGCAAA & mmu-mir-19a-3p \\
\hline & & mmu-mir-301b-3p & & & mmu-mir-19b-3p \\
\hline $\operatorname{miR}-130 a-5 p$ & CUCUUUU & mmu-mir-130a-5p & miR-204-5p & UCCCUUU & mmu-mir-211-5p \\
\hline miR-130b-5p & CUCUUUC & mmu-mir-130b-5p & miR-21-5p & AGCUUAU & mmu-mir-21a-5p \\
\hline $\operatorname{miR}-132-3 p$ & AACAGUC & mmu-mir-132-3p & $\operatorname{miR}-210-3 p$ & UGUGCGU & mmu-mir-210-3p \\
\hline miR-132-5p & ACCGUGG & mmu-mir-132-5p & miR-210-5p & GCCACUG & mmu-mir-210-5p \\
\hline $\operatorname{miR}-135 a-5 p$ & AUGGCUU & mmu-mir-135a-5p & miR-219a-5p & GAUUGUC & mmu-mir-219a-5p \\
\hline miR-138-5p & GCUGGUG & mmu-mir-138-5p & $\operatorname{miR}-22-3 p$ & AGCUGCC & mmu-mir-22-3p \\
\hline miR-139-5p & CUACAGU & mmu-mir-139-5p & $\operatorname{miR}-22-5 p$ & GUUCUUC & mmu-mir-22-5p \\
\hline miR-140-3p & ACCACAG & mmu-mir-140-3p & $\operatorname{miR}-221-3 p$ & GCUACAU & mmu-mir-222-3p \\
\hline miR-140-5p & AGUGGUU & mmu-mir-140-5p & $\operatorname{miR}-223-3 p$ & GUCAGUU & mmu-mir-223-3p \\
\hline miR-142-3p & GUAGUGU & mmu-mir-142a-3p & $\operatorname{miR}-224-5 p$ & AAGUCAC & mmu-mir-224-5p \\
\hline miR-143-5p & & mmu-mir-143-5p & $\operatorname{miR}-23 a-3 p$ & UCACAUU & mmu-mir-23a-3p \\
\hline $\operatorname{miR}-144-3 p$ & ACAGUAU & mmu-mir-144-3p & & & mmu-mir-23b-3p \\
\hline \multirow[t]{2}{*}{ miR-144-5p } & GAUAUC & mmu-mir-144-5p & miR-24-1-5p & UGCCUAC & mmu-mir-24-2-5p \\
\hline & & & miR-24-3p & GGCUCAG & mmu-mir-24-3p \\
\hline \multirow[t]{2}{*}{$\operatorname{miR}-145-5 p$} & UCCAGUU & mmu-mir-145a-5p & miR-24-1-5p & UGCCUAC & mmu-mir-24-2-5p \\
\hline & & & & & Continued \\
\hline
\end{tabular}




\begin{tabular}{|c|c|c|c|c|c|}
\hline Symbol & Seed regio & ID & Symbol & Seed regio & ID \\
\hline miR-24-3p & GGCUCAG & mmu-mir-24-3p & miR-34a-5p & GGCAGUG & mmu-mir-34a-5p \\
\hline miR-26a-5p & UCAAGUA & mmu-mir-26a-5p & & & mmu-mir-34b-5p \\
\hline \multirow[t]{2}{*}{$\operatorname{miR}-26 a-5 p$} & UCAAGUA & mmu-mir-26a-5p & & & mmu-mir-34c-5p \\
\hline & & mmu-mir-26b-5p & miR-350 & UCACAAA & mmu-mir-350-3p \\
\hline \multirow[t]{2}{*}{$\operatorname{miR}-27 a-3 p$} & UCACAGU & mmu-mir-27a-3p & $\operatorname{miR}-361-5 p$ & UAUCAGA & mmu-mir-361-5p \\
\hline & & mmu-mir-27b-3p & $\operatorname{miR}-362-5 p$ & AUCCUUG & mmu-mir-362-5p \\
\hline miR-29a-5p & CUGAUUU & mmu-mir-29a-5p & miR-374b-5p & UAUAAUA & mmu-mir-374b-5p \\
\hline miR-29b-1-5p & CUGGUUU & mmu-mir-29b-1-5p & $\operatorname{miR}-378 a-3 p$ & CUGGACU & mmu-mir-378a-3p \\
\hline \multirow[t]{3}{*}{ miR-29b-3p } & AGCACCA & mmu-mir-29a-3p & & & mmu-mir-378c \\
\hline & & mmu-mir-29b-3p & miR-378a-5p & UCCUGAC & mmu-mir-378a-5p \\
\hline & & mmu-mir-29c-3p & miR-3909 & GUCCUCU & mmu-mir-877-3p \\
\hline $\operatorname{miR}-3065-5 p$ & CAACAAA & mmu-mir-3065-5p & $\operatorname{miR}-423-3 p$ & GCUCGGU & mmu-mir-423-3p \\
\hline \multirow[t]{5}{*}{$\operatorname{miR}-30 c-5 p$} & GUAAACA & mmu-mir-30a-5p & $\operatorname{miR}-423-5 p$ & GAGGGGC & mmu-mir-423-5p \\
\hline & & mmu-mir-30b-5p & $\operatorname{miR}-425-5 p$ & AUGACAC & mmu-mir-425-5p \\
\hline & & mmu-mir-30c-5p & miR-451a & AACCGUU & mmu-mir-451a \\
\hline & & mmu-mir-30d-5p & $\operatorname{miR}-501-5 p$ & AUCCUUU & mmu-mir-501-5p \\
\hline & & mmu-mir-30e-5p & miR-503-5p & AGCAGCG & mmu-mir-503-5p \\
\hline $\operatorname{miR}-31-3 p$ & GCUAUGC & mmu-mir-31-3p & $\operatorname{miR}-532-5 p$ & AUGCCUU & mmu-mir-532-5p \\
\hline $\operatorname{miR}-31-5 p$ & GGCAAGA & mmu-mir-31-5p & $\operatorname{miR}-542-3 p$ & GUGACAG & mmu-mir-542-3p \\
\hline miR-3176 & CUGGCCU & mmu-mir-378d & $\operatorname{miR}-574-5 p$ & GAGUGUG & mmu-mir-574-5p \\
\hline miR-324-5p & GCAUCCC & mmu-mir-324-5p & $\operatorname{miR}-582-5 p$ & UACAGUU & mmu-mir-582-5p \\
\hline $\operatorname{miR}-328-3 p$ & UGGCCCU & mmu-mir-328-3p & miR-652-3p & AUGGCGC & mmu-mir-652-3p \\
\hline miR-329-3p & ACACACC & mmu-mir-362-3p & miR-670-5p & UCCCUGA & mmu-mir-670-5p \\
\hline $\operatorname{miR}-33-5 p$ & UGCAUUG & mmu-mir-33-5p & miR-700-5p & AAGGCUC & mmu-mir-700-5p \\
\hline miR-330-5p & CUCUGGG & mmu-mir-326-3p & $\operatorname{miR}-744-3 p$ & UGUUGCC & mmu-mir-744-3p \\
\hline miR-331-3p & CCCCUGG & mmu-mir-331-3p & miR-744-5p & & mmu-mir-744-5p \\
\hline miR-339-5p & CCCUGUC & mmu-mir-339-5p & $\operatorname{miR}-7 a-5 p$ & GGAAGAC & mmu-mir-7a-5p \\
\hline $\operatorname{miR}-340-3 p$ & CCGUCUC & mmu-mir-340-3p & $\operatorname{miR}-872-3 p$ & GAACUAU & mmu-mir-872-3p \\
\hline $\operatorname{miR}-344 a-5 p$ & CAGGCUC & mmu-mir-484 & $\operatorname{miR}-872-5 p$ & AGGUUAC & mmu-mir-872-5p \\
\hline $\operatorname{miR}-345-5 p$ & CUGACCC & mmu-mir-345-5p & miR-9-5p & CUUUGGU & mmu-mir-9-5p \\
\hline $\operatorname{miR}-3473 b$ & GGCUGGA & mmu-mir-3473b & $\operatorname{miR}-92 a-3 p$ & AUUGCAC & mmu-mir-25-3p \\
\hline $\operatorname{miR}-34 c-3 p$ & AUCACUA & $\begin{array}{l}\text { mmu-mir-3473e } \\
\text { mmu-mir-34b-3p }\end{array}$ & & & $\begin{array}{l}\text { mmu-mir-32-5p } \\
\text { mmu-mir-92a-3p }\end{array}$ \\
\hline
\end{tabular}




\begin{tabular}{|c|c|c|}
\hline ID & Symbol & Entrez Gene Name \\
\hline Q640N1 & AEBP1 & AE binding protein 1 \\
\hline P48036 & ANXA5 & annexin A5 \\
\hline P08226 & APOE & apolipoprotein E \\
\hline Q3TWT5 & ASAH1 & $\mathrm{N}$-acylsphingosine amidohydrolase 1 \\
\hline Q3TXF9 & ATP1A1 & ATPase $\mathrm{Na}+/ \mathrm{K}+$ transporting subunit alpha 1 \\
\hline O55107 & BSG & basigin (Ok blood group) \\
\hline Q9WVT6 & CA14 & carbonic anhydrase 14 \\
\hline P41731 & CD63 & CD63 molecule \\
\hline P35762 & CD81 & CD81 molecule \\
\hline P29812 & DCT & dopachrome tautomerase \\
\hline Q3UAM9 & ENG & endoglin \\
\hline P17182 & ENO1 & enolase 1 \\
\hline P19096 & FASN & fatty acid synthase \\
\hline P11276 & FN1 & fibronectin 1 \\
\hline P09528 & FTH1 & ferritin heavy chain 1 \\
\hline P16858 & GAPDH & glyceraldehyde-3-phosphate dehydrogenase \\
\hline P08752 & GNAI2 & G protein subunit alpha i2 \\
\hline Q3TAV1 & GPNMB & glycoprotein $\mathrm{nmb}$ \\
\hline P19157 & GSTP1 & glutathione S-transferase pi 1 \\
\hline P11499 & HSP90AB1 & heat shock protein 90 alpha family class B member 1 \\
\hline B1B0C7 & HSPG2 & heparan sulfate proteoglycan 2 \\
\hline P02468 & LAMC1 & laminin subunit gamma 1 \\
\hline Q3U2W5 & LGALS8 & galectin 8 \\
\hline P21956 & MFGE8 & milk fat globule-EGF factor 8 protein \\
\hline P62962 & PFN1 & profilin 1 \\
\hline P52480 & PKM & pyruvate kinase, muscle \\
\hline P17742 & PPIA & peptidylprolyl isomerase A \\
\hline Q61171 & PRDX2 & peroxiredoxin 2 \\
\hline Q8CCG5 & RALB & RAS like proto-oncogene B \\
\hline O08992 & SDCBP & syndecan binding protein \\
\hline Q0VGP2 & SEMA3B & semaphorin 3B \\
\hline P10852 & SLC3A2 & solute carrier family 3 member 2 \\
\hline Q3UQM7 & SLC7A5 & solute carrier family 7 member 5 \\
\hline O09044 & SNAP23 & synaptosome associated protein 23 \\
\hline Q64337 & SQSTM1 & sequestosome 1 \\
\hline Q542D9 & TFRC & transferrin receptor \\
\hline P39876 & TIMP3 & TIMP metallopeptidase inhibitor 3 \\
\hline Q9R1Q6 & TMEM176B & transmembrane protein 176B \\
\hline Q3UCW0 & TSG101 & tumor susceptibility 101 \\
\hline
\end{tabular}




\begin{tabular}{|c|c|c|}
\hline Symbol & Seed regio & ID \\
\hline \multirow[t]{2}{*}{ let-7a-3p } & UAUACAA & mmu-let-7a-1-3p, mmu-let-7b-3p \\
\hline & & mmu-let-7c-2-3p, mmu-let-7f-1-3p \\
\hline \multirow[t]{4}{*}{ let-7a-5p } & GAGGUAG & mmu-let-7a-5p, mmu-let-7b-5p \\
\hline & & mmu-let-7c-5p, mmu-let-7d-5p \\
\hline & & mmu-let-7e-5p, mmu-let-7f-5p \\
\hline & & mmu-let-7g-5p, mmu-mir-98-5p \\
\hline $\operatorname{miR}-100-5 \mathrm{p}$ & ACCCGUA & mmu-mir-99a-5p, mmu-mir-99b-5p \\
\hline \multirow[t]{2}{*}{$\operatorname{miR}-125 b-5 p$} & CCCUGAG & mmu-mir-125a-5p, mmu-mir-125b-5p \\
\hline & & mmu-mir-351-5p \\
\hline miR-126a-5p & AUUAUUA & mmu-mir-126a-5p \\
\hline miR-139-5p & CUACAGU & mmu-mir-139-5p \\
\hline \multirow[t]{3}{*}{$\operatorname{miR}-16-5 p$} & AGCAGCA & mmu-mir-15a-5p, mmu-mir-15b-5p \\
\hline & & mmu-mir-16-5p, mmu-mir-195a-5p \\
\hline & & mmu-mir-322-5p, mmu-mir-497a-5p \\
\hline \multirow[t]{2}{*}{$\operatorname{miR}-17-5 p$} & AAAGUGC & mmu-mir-106b-5p, mmu-mir-17-5p \\
\hline & & mmu-mir-20a-5p, mmu-mir-93-5p \\
\hline \multirow[t]{2}{*}{ miR-181a-5p } & ACAUUCA & mmu-mir-181a-5p, mmu-mir-181b-5p \\
\hline & & mmu-mir-181c-5p, mmu-mir-181d-5p \\
\hline $\operatorname{miR}-18 \mathrm{a}-5 \mathrm{p}$ & AAGGUGC & mmu-mir-18a-5p \\
\hline miR-193a-3p & ACUGGCC & mmu-mir-193a-3p \\
\hline miR-199a-3p & CAGUAGU & mmu-mir-199a-3p, mmu-mir-199b-3p \\
\hline miR-199a-5p & CCAGUGU & mmu-mir-199a-5p, mmu-mir-199b-5p \\
\hline \multirow[t]{2}{*}{$\operatorname{miR}-19 b-3 p$} & GUGCAAA & mmu-mir-19a-3p, \\
\hline & & mmu-mir-19b-3p \\
\hline $\operatorname{miR}-21-5 p$ & AGCUUAU & mmu-mir-21a-5p \\
\hline miR-221-3p & GCUACAU & mmu-mir-222-3p \\
\hline miR-223-3p & GUCAGUU & mmu-mir-223-3p \\
\hline \multirow[t]{2}{*}{$\operatorname{miR}-23 a-3 p$} & UCACAUU & mmu-mir-23a-3p \\
\hline & & mmu-mir-23b-3p \\
\hline \multirow[t]{5}{*}{$\operatorname{miR}-30 c-5 p$} & GUAAACA & mmu-mir-30a-5p \\
\hline & & mmu-mir-30b-5p \\
\hline & & mmu-mir-30c-5p \\
\hline & & mmu-mir-30d-5p \\
\hline & & mmu-mir-30e-5p \\
\hline miR-330-5p & CUCUGGG & mmu-mir-326-3p \\
\hline \multirow[t]{3}{*}{$\operatorname{miR}-34 a-5 p$} & GGCAGUG & mmu-mir-34a-5p \\
\hline & & mmu-mir-34b-5p \\
\hline & & mmu-mir-34c-5p \\
\hline $\operatorname{miR}-7 a-5 p$ & GGAAGAC & mmu-mir-7a-5p \\
\hline
\end{tabular}




\section{OPEN ACCESS}

Edited by:

Fabrizio Mattei,

National Institute of Health (ISS), Italy

Reviewed by:

Kawaljit Kaur

University of California, Los Angeles,

United States

Fatemeh Momen-Heravi,

Columbia University, United States

*Correspondence:

Krisztina Buzás

kr.buzas@gmail.com

Specialty section:

This article was submitted to Cancer Immunity and Immunotherapy,

a section of the journal

Frontiers in Immunology

Received: 21 March 2019 Accepted: 01 October 2019 Published: 18 October 2019

Citation:

Gyukity-Sebestyén E, Harmati M,

Dobra G, Németh IB, Mihály J, Zvara Á, Hunyadi-Gulyás É, Katona $R$,

Nagy I, Horváth P, Bálind Á,

Szkalisity Á, Kovács M, Pankotai T,

Borsos B, Erdélyi M, Szegletes Z,

Veréb ZJ, Buzás El, Kemény L, Bíró T and Buzás K (2019)

Melanoma-Derived Exosomes Induce

PD-1 Overexpression and Tumor

Progression via Mesenchymal Stem

Cell Oncogenic Reprogramming.

Front. Immunol. 10:2459.

doi: 10.3389/fimmu.2019.02459

\section{Melanoma-Derived Exosomes Induce PD-1 Overexpression and Tumor Progression via Mesenchymal Stem Cell Oncogenic Reprogramming}

Edina Gyukity-Sebestyén ${ }^{1,2}$, Mária Harmati ${ }^{1,2}$, Gabriella Dobra ${ }^{1,2}$, István B. Németh ${ }^{3}$, Johanna Mihály ${ }^{4}$, Ágnes Zvara ${ }^{5}$, Éva Hunyadi-Gulyás ${ }^{6}$, Róbert Katona ${ }^{7}$, István Nagy ${ }^{8}$, Péter Horváth ${ }^{1}$, Árpád Bálind ${ }^{1}$, Ábel Szkalisity ${ }^{1}$, Mária Kovács ${ }^{1,9}$, Tibor Pankotai ${ }^{10}$, Barbara Borsos ${ }^{10}$, Miklós Erdélyi ${ }^{11}$, Zsolt Szegletes ${ }^{12}$, Zoltán J. Veréb ${ }^{3}$, Edit l. Buzás ${ }^{13}$, Lajos Kemény ${ }^{3}$, Tamás Bíró ${ }^{4}$ and Krisztina Buzás ${ }^{1,14 *}$

1 Laboratory of Microscopic Image Analysis and Machine Learning, Institute of Biochemistry, Biological Research Centre of Hungarian Academy of Sciences, Szeged, Hungary, ${ }^{2}$ Doctoral School of Interdisciplinary Sciences, Faculty of Medicine, University of Szeged, Szeged, Hungary, ${ }^{3}$ Department of Dermatology and Allergology, University of Szeged, Szeged, Hungary, ${ }^{4}$ Department of Immunology, Faculty of Medicine, University of Debrecen, Debrecen, Hungary, ${ }^{5}$ Laboratory of Functional Genomics, Institute of Genetics, Biological Research Centre of Hungarian Academy of Sciences, Szeged, Hungary, ${ }^{6}$ Laboratory of Proteomics Research, Institute of Biochemistry, Biological Research Centre of Hungarian Academy of Sciences, Szeged, Hungary, ${ }^{7}$ Artificial Chromosome and Stem Cell Research Laboratory, Institute of Genetics, Biological Research Centre of Hungarian Academy of Sciences, Szeged, Hungary, ${ }^{8}$ Sequencing Platform, Institute of Biochemistry, Biological Research Centre of Hungarian Academy of Sciences, Szeged. Hungary, ${ }^{9}$ Department of Pharmacology and Pharmacotherapy, Faculty of Medicine, University of Szeged, Szeged, Hungary, ${ }^{10}$ Department of Biochemistry and Molecular Biology, Faculty of Science and Informatics, University of Szeged, Szeged, Hungary, ${ }^{11}$ Advanced Optical Imaging Group, Department of Optics and Quantum Electronics, Faculty of Science and Informatics, University of Szeged, Szeged, Hungary, ${ }^{12}$ Atomic Force Microscope Laboratory, Institute of Biophysics, Biological Research Centre of Hungarian Academy of Sciences, Szeged, Hungary, ${ }^{13}$ MTA-SE Immuno-proteogenomics Extracellular Vesicle Research Group, Department of Genetics, Cell- and Immunobiology, Faculty of Medicine, Semmelweis University, Budapest, Hungary, ${ }^{14}$ Department of Oral Biology and Experimental Dental Research, Faculty of Dentistry, University of Szeged, Szeged, Hungary

Recently, it has been described that programmed cell death protein 1 (PD-1) overexpressing melanoma cells are highly aggressive. However, until now it has not been defined which factors lead to the generation of PD-1 overexpressing subpopulations. Here, we present that melanoma-derived exosomes, conveying oncogenic molecular reprogramming, induce the formation of a melanoma-like, PD-1 overexpressing cell population (mMSC ${ }^{\mathrm{PD}-1+}$ ) from naïve mesenchymal stem cells (MSCs). Exosomes and $\mathrm{mMSC}^{\mathrm{PD}-1+}$ cells induce tumor progression and expression of oncogenic factors in vivo. Finally, we revealed a characteristic, tumorigenic signaling network combining the upregulated molecules (e.g., PD-1, MET, RAF1, BCL2, MTOR) and their upstream exosomal regulating proteins and miRNAs. Our study highlights the complexity of exosomal communication during tumor progression and contributes to the detailed understanding of metastatic processes.

Keywords: PD-1, exosome, melanoma/tumor progression, stem cell, reprogramming, signalization pattern, metastasis 


\section{INTRODUCTION}

Heterogeneous tumor tissue is comprised of a wide variety of collocated cells. Their spatiotemporal co-existence facilitates direct communication between them. Cancer cells contribute to tumor niche formation not only by soluble factor production and receptor-ligand interactions (1), but also by releasing vesicles whose molecular contents add up to a complex information package. Previous studies demonstrated that cultured human tumor cells release extracellular vesicles such as exosomes of 20$120 \mathrm{~nm}$ diameters (2). Among others, exosomes carry structural and signaling proteins, MHC molecules, cell surface molecules typically associated with apoptosis, and mRNAs/miRNAs with multiple functions (3). Therefore, these exosomal-molecularpatterns, as unique entities of the complex intercellular communication, are not independent of the quality or state of the mother cell $(4,5)$. Exosomes have been recognized long ago, but their identification, characterization, and isolation are still under intense investigation. Further, whereas the definition of exosomes is based on the pathway of biogenesis, oncosomes form a functional class of extracellular vesicles. Indeed, oncosomes are suggested being capable of carrying and conveying tumor-related information (6) and might have a significant role in formation of tumor microenvironment (7).

Mesenchymal stem cells (MSCs) were first described as stromal cells of the bone marrow with multipotent differentiation potential and characteristic immunomodulatory effects (8). In relation to their immunological and differentiation properties, there is a debate about the role of MSCs in tumor progression (9). Indeed, the cellular fate could depend on the cancer type and also on the status of the affected MSCs. Activated MSCs can secrete pro-angiogenic soluble factors and are able to differentiate to vessel wall associated cells (10) or to cancer associated fibroblasts (CAFs) (11). Furthermore, Baglio et al. showed that tumor secreted extracellular vesicles promote osteosarcoma progression via TGF $\beta$ signaling induced IL-6 production by MSCs (12). Moreover, Peinado et al. demonstrated that exosomes from highly metastatic melanomas increase the metastatic behavior of primary tumors by permanently educating bone marrow progenitors through the receptor tyrosine kinase MET (13).

Malignant melanoma is a highly invasive, metastatic cancer with poor prognosis and survival rate. Since melanoma has been long recognized as a highly immunogenic tumor, therapeutic approaches target different immunological mechanisms to treat patients with this aggressive skin cancer. In the past decade, besides IL-2 and CTLA-4 therapies, the PD-1:PD-L1 blockade proved to be an effective treatment in metastatic melanoma (14). Programmed cell death protein 1 (PD-1), mainly expressed by immune cells such as activated $\mathrm{T}$ cells, dendritic cells and macrophages is a cell surface receptor with a central role in modulation of $\mathrm{T}$ cell responses. PD-1 binding to its ligand, PD-L1, can result in apoptosis and anergy of immune cells.

Notably, PD-1 expression is not a default property of melanoma cells. Of further importance, recently Kleffel et al. demonstrated that PD-1 overexpressing melanoma cell subpopulations are especially aggressive, and that melanoma PD-1:PD-L1 interactions modulate mTOR signaling, whereby they interfere with programmed cell death (15). This work is remarkably instrumental with respect to the consequences of PD-1 overexpression and experimental inhibition of PD-1 functions (Pdcd1-shRNA or anti-PD-1 antibody). However, it does not define the factors the activation of which lead to the generation of PD-1 overexpressing subpopulations. This question, though, is an especially intriguing one, if one considers that one of the most effective immunotherapies available today is based on the blockade of the aforementioned PD-1:PD-L1 interaction (16).

Based on the above, in the current study, we aimed at specifically and systematically addressing the following questions:

1. Can we unambiguously define the cellular and molecular signs of melanoma-derived exosome-induced, intercellular communication-mediated malignant transformation of MSC cultures?

2. Can we detect the melanoma-derived exosome-induced tumor progression in vivo?

3. What alteration can be detected in the expression of PD-1, a melanoma progression marker and therapeutic target, upon exposure to melanoma-derived exosomes?

Here, we present the first piece of evidence that melanoma exosomes, by conveying a complex oncogenic molecular reprogramming, induce the formation of a PD-1 overexpressing cell population (melanoma-like $\mathrm{MSC}^{\mathrm{PD}-1+} ; \mathrm{mMSC}^{\mathrm{PD}-1+}$ ) from naïve MSCs. These $\mathrm{mMSC}^{\mathrm{PD}-1+}$ cells represent a new entity with melanoma-like gene expression profile and phenotypic properties. Further, exosomes and exosomeactivated $\mathrm{mMSC}^{\mathrm{PD}-1+}$ cells induce rapid tumor progression in vivo, due to their strong expression of oncogenic dominance factors and decreased susceptibility to programmed cell death.

\section{MATERIALS AND METHODS}

\section{Cell Culture and Cell Line}

B16F1 mouse melanoma cell line was obtained from ECACC and cultured in Dulbecco's modified Eagle's medium (DMEM, Lonza, Basel, Switzerland) containing 10\% fetal bovine serum (FBS) (Euroclone, Milano, Italy), $1 \%$ sodium pyruvate, $1 \%$ MEM non-essential amino acids, 1\% MEM vitamin solution, $2 \mathrm{mM}$ L-glutamine, and 1\% Penicillin-Streptomycin-Amphotericin B Mixture (all from Lonza).

Murine MSCs were isolated from C57BL/6N (Charles River Laboratories, Sulzfeld) 8 week-old male mice (17). Abdominal inguinal fat pads were excised, rinsed with RPMI 1640 medium, transferred to sterile tissue culture dishes and mechanically dissociated. The dissociated tissue was resuspended in RPMI 1640 containing $100 \mu \mathrm{g} / \mathrm{ml}$ collagenase (Sigma-Aldrich, St. Louis, $\mathrm{MO}$ ) and incubated at $37^{\circ} \mathrm{C}$ for $50 \mathrm{~min}$. Collagenase was neutralized with a growth medium containing $10 \%$ FBS. After centrifugation at $470 \mathrm{~g}$ for $15 \mathrm{~min}$, cell pellets were resuspended and washed in the culture medium. After the centrifugation, cell pellets were resuspended in complete MesenCult medium (Stemcell Technologies, Vancouver, British Columbia, Canada) and filtered through a $100 \mu \mathrm{m}$ cell strainer (BD Biosciences, 
Franklin Lakes, NJ) to tissue culture dishes, and cultured for $48 \mathrm{~h}$ at $37^{\circ} \mathrm{C}$ in $5 \% \mathrm{CO}_{2}$ and $90 \%$ humidity. Unattached cells and debris were then removed and fresh medium was added to the adherent cells. The cells were cultured to $80 \%$ confluence before being released with trypsin-EDTA and sub-cultured.

Purity of MSC cultures were checked by flow cytometry analysis using the Mouse Multipotent Mesenchymal Stromal Cell Marker Antibody Panel (R\&D Systems, Minneapolis, MN) according to the manufacturer's instructions.

\section{Isolation and Characterization of the Melanoma-Derived Exosomes Exosome Isolation by Filtration and Differential Centrifugation}

Exosomes were isolated by adapting the protocol of Peinado et al. (13) from melanoma cell culture supernatant. B16F1 supernatants were harvested, supplemented by complete protease inhibitor cocktail (Roche, Mannheim, Germany) and centrifuged at $780 \mathrm{~g}$ for $5 \mathrm{~min}$ at $4^{\circ} \mathrm{C}$ to remove intact cells. Then, the supernatants were centrifuged at $3,900 \mathrm{~g}$ for $15 \mathrm{~min}$ at $4^{\circ} \mathrm{C}$ and filtered through a $0.2 \mu \mathrm{m}$ membrane (Millipore, Billerica, MA) to remove larger cell fragments and microvesicles. Exosomes were pelleted by ultracentrifugation at $150,000 \mathrm{~g}$ (T-1270 rotor at $40,500 \mathrm{rpm}$ ) for $1 \mathrm{~h}$ at $4^{\circ} \mathrm{C}$. The pellet was washed twice and resuspended in DPBS and stored at $-80^{\circ} \mathrm{C}$.

The concentration of exosomal proteins was determined using a Pierce BCA Protein assay kit (Thermo Fisher Scientific, Waltham, MA) and a Benchmark Microplate Reader (Bio-Rad, Hercules, CA) according to the manufacturer's instructions.

\section{Determination of Shape and Size of the Isolated Vesicles}

\section{Scanning electron microscopy (SEM)}

Five microliters of isolated exosomes in DPBS were spotted on glass coverslips coated with $0.01 \%$ Poly-L-Lysine (SigmaAldrich) and incubated overnight at $4^{\circ} \mathrm{C}$. Using sterile forceps, coverslips were transferred into a plastic plate. Exosomes were fixed by gently adding 2\% paraformaldehyde buffer $\mathrm{pH} 7.2$ diluted in DPBS for $30 \mathrm{~min}$. The coverslips were washed twice with DPBS and dehydrated with a graded ethanol series (40, 60 , 80, and $100 \%$ ethanol, each for $5 \mathrm{~min}$ ). The samples were dried with a critical point dryer (QUORUM K850, Quorum Technologies Ltd, Laughton, UK) and the coverslips were mounted onto a microscope stub at a time using carbon tape, followed by $7 \mathrm{~nm}$ gold coating (QUORUM Q150, Quorum Technologies Ltd) and observed under a field-emission scanning electron microscope (JEOL JSM-7100F/LV).

\section{Atomic force microscopy (AFM)}

Exosomes were let to adsorb to the freshly cleaved muscovite mica (SPI-ChemTM Mica Sheets, Structure Probe, Inc., West Chester, PA) surface directly from DPBS. All AFM measurements were carried out with an Asylum MFP-3D head and Molecular Force Probe Controller (Asylum Research, Santa Barbara, CA), using Asylum Research MFP-3D program (version 15.09.112) written in Igor Pro software (version 6.37, Wavemetrics, Lake Oswego, OR). Image procession and data calculation were made using the same software. Silicon rectangular cantilevers (OMLCAC240TS, Olympus Optical Co., Ltd., Tokyo, Japan) with the typical spring constant of $2 \mathrm{~N} / \mathrm{m}$ were used in dry condition and silicon nitride rectangular cantilevers with "V" shaped tips (BioLever BL-RC150VB, Olympus Optical Co. Ltd.) with the typical spring constant of $0.03 \mathrm{~N} / \mathrm{m}$ in buffer solution. Typically, 512 $\times 512$ points was taken at 1 line/s scan rate. The measurements presented here are amplitude images and height profile.

\section{Detection of Exosomal Markers by Western Blotting}

Protein samples were resuspended in 4x sample buffer (NuPAGE LDS Sample Buffer (4X), NuPAGE Sample Reducing Agent (10X), Thermo Fisher Scientific), boiled at $96^{\circ} \mathrm{C}$ for $10 \mathrm{~min}$, and immediately cooled on ice. Electrophoresis of the proteins was performed using 4-12\% Bis-Tris Protein Gels (NuPAGE Novex, Thermo Fisher Scientific), ProSieve Color Protein Markers (Lonza), and electrophoresis buffer (NuPAGE MOPS SDS Running Buffer, Thermo Fisher Scientific) at $200 \mathrm{~V}$ and $0.03 \mathrm{~A}$ for $40 \mathrm{~min}$.

The proteins of electrophoresed gels were transferred to Immobilon transfer membrane (Millipore, Darmstadt, Germany) using transfer buffer [NuPAGE Transfer Buffer (20X)] at $30 \mathrm{~V}$ and $170-110 \mathrm{~mA}$ for $60 \mathrm{~min}$. Membranes were blocked in TBST buffer $(25 \mathrm{mM}$ Tris- $\mathrm{HCl}, 150 \mathrm{mM} \mathrm{NaCl}, 0.05 \%$ Tween-20, $\mathrm{pH}$ 7.2 ) containing $5 \%$ non-fat milk for $60 \mathrm{~min}$ at room temperature (RT). After blocking, membranes were incubated with each primary antibody in TBST buffer containing $1 \%$ non-fat milk overnight at $4^{\circ} \mathrm{C}$. Membranes were washed three times for $10 \mathrm{~min}$ with TBST buffer and incubated for $60 \mathrm{~min}$ at room temperature with secondary antibody in TBST buffer containing $1 \%$ nonfat milk. Membranes were washed three times for $10 \mathrm{~min}$ with TBST buffer.

For exosomal marker identification western blot analyses were performed with an anti-CD63 polyclonal antibody (1:250 dilution; Biorbyt, Cambridge, UK) anti-CD9 monoclonal antibody (clone EPR2949, 1:500 dilution, LifeSpan Biosciences, Seattle, WA), anti-CD81 monoclonal antibody (clone: EAT2, 1:1,000 dilution, LifeSpan Biosciences, Seattle, WA), and anti-HSP70 monoclonal antibody (clone: C92F3A-5, 1:8,000 dilution; Enzo Life Sciences, Farmingdale, NY), anti-rabbit IgG HRP-Conjugated antibody (1:1,000 dilution, R\&D Systems), anti-Hamster IgG HRP-Conjugated antibody (1:30,000 dilution, Thermo Fisher Scientific). Bound antibodies were visualized by chemiluminescence using an ECL Plus Western Blotting detection system (Advansta, Menlo Park, CA). Immunoreactive signals were detected by using LI-COR ODYSSEY Fc (Dual-mode imaging system) imager followed by analysis with Odyssey v1.2, Image Studio Lite v5.2.

\section{Identification of Exosomal Proteins by Mass Spectrometry}

Exosomal proteins $(24 \mu \mathrm{g})$ were separated in $4-12 \%$ Bis-Tris Protein Gels and stained with Coomassie blue (0.1\% Coomassie Brilliant Blue R-250, 50\% methanol and 10\% acetic acid).

Each lane was cut to 12-12 equal bands and subjected to ingel digestion. Gel bands were diced to smaller pieces, and the SDS and CBB dyes were washed out with $3 \times 50 \mu l 25 \mathrm{mM}$ 
ammonium-bicarbonate $(\mathrm{ABC}) / 50 \%$ Acetonitrile $(\mathrm{AcN})$. After reduction with DTT (1,4-dithiothreitol, Sigma-Aldrich; $20 \mu \mathrm{l}$, $10 \mathrm{mM}$ DTT in $25 \mathrm{mM} \mathrm{ABC}$ ) at $56^{\circ} \mathrm{C}$ for $30 \mathrm{~min}$, and alkylation with IAM (iodoacetamide, Sigma- Aldrich; $20 \mu \mathrm{l}, 55 \mathrm{mM}$ IAM in $25 \mathrm{mM} \mathrm{ABC}$ ) at RT in the dark for $30 \mathrm{~min}$, the gel samples were dried in a vacuum centrifuge and after that rehydrated in $20 \mu \mathrm{l}$ of trypsin (Sequencing Grade Modified Trypsin, Promega, Fitchburg, WI; $5 \mathrm{ng} / \mu \mathrm{l}$ in $25 \mathrm{mM} \mathrm{ABC}$ ) and incubated at $37^{\circ} \mathrm{C}$. The digestion was stopped after $4 \mathrm{~h}$ by lowering the $\mathrm{pH}$ of the buffer below 3 , by adding $2 \mu \mathrm{l}$ of $10 \%$ formic acid. Tryptic peptides were extracted from the gel with $3 \times 50 \mu$ of $2 \%$ formic acid in $50 \% \mathrm{AcN}$ and dried. Prior mass spectrometric analysis, all samples were redissolved in 50-50 $\mathrm{kl}$ of $0.1 \%$ formic acid (FA).

Samples were analyzed on an LTQ-Orbitrap Elite (Thermo Fisher Scientific) mass spectrometer on-line coupled with a nanoHPLC (nanoAcquity, Waters, Milford, MA) system. 5-5 $\mathrm{ll}$ of the in-gel digests were loaded (for $3 \mathrm{~min}$ at $8 \mu \mathrm{l} / \mathrm{min}$ flow, using $0.1 \% \mathrm{FA}$ in $3 \%$ Acetonitrile-97\% water) onto a reversed phase trap column (Waters, Symmetry C18, $0.180 \times 20 \mathrm{~mm}$ ) and separated on a C18 reversed phase (Waters, Milford, MA, BEH300C18 $1.7 \mu \mathrm{m})$ nanocolumn $(0.075 \times 200 \mathrm{~mm})$. The flow rate was $330 \mathrm{nl} / \mathrm{min}$ and a linear gradient was used from 3 to $40 \%$ $\mathrm{B}$ in $37 \mathrm{~min}$ (solvent $\mathrm{A}$ was $0.1 \% \mathrm{FA}$ in water and solvent $\mathrm{B}$ was $0.1 \%$ FA in Acetonitrile).

The high voltage $(1.2 \mathrm{kV})$ was applied through liquid junction between the chromatographic column and the non-coated silica nanospray emitter (NewObjective, Woburn, MA, $10 \mu \mathrm{m}$ tip ID). The mass spectrometer operated in data-dependent mode: the survey mass spectra were detected in the orbitrap with high resolution $(\mathrm{R}=60 \mathrm{k} @ \mathrm{~m} / \mathrm{z}$ : 400, mass range $\mathrm{m} / \mathrm{z}$ : 380 $1,400)$ and the most abundant multiply charged 20 peaks were selected for ion-trap fragmentation (NCE: $35 \%$; activation q: 0.25 ; activation time: $10 \mathrm{~ms}$; minimum signal intensity: 5,000 counts). The MSMS spectra were detected in the ion trap. Dynamic exclusion was used, the precursors were excluded for $15 \mathrm{~s}$ after the first fragmentation event.

Data analysis: searchable peaklists (mgf format) were extracted using ProteomeDiscoverer (ver:1.4 Thermo Fisher Scientific) and subjected to database search on our in-house ProteinProspector (ver: 5.14.1) search engine using the following parameters: parent ion tolerant: $5 \mathrm{ppm}$; fragment ion tolerant: 0.6 Da; Cys carbamidomethylation was set as constant and Met oxidation, cyclisation of peptide N-terminal Glu to pyroglutamic acid, protein $\mathrm{N}$ terminal acetylation were set as variable modifications. Only fully tryptic peptides were considered with maximum of 2 missed cleavage sites. The Mus musculus and Bos taurus protein sequences of the Uniprot (UniProtKB.06.11.2014) database completed with human keratins and pig trypsin, altogether 106,330 protein sequences were searched. For the false discovery rate (FDR) estimation, the searches were performed on the database concatenated with the randomized sequences. Protein identification was accepted if the ProteinProspector expectation value was $<0.01$ and the protein was identified with at least 2 unique peptides (expectation value $<0.05$ and score higher than 15). FDR values were $<1 \%$ in all cases.
For functional validation, the resulted protein list was analyzed by the "Core Analysis" function included in Ingenuity Pathway Analysis (IPA, Quiagen Bioinformatics) software.

\section{Isolation and Detection of Exosomal miRNAs}

miRNA sequencing was performed using SOLiD Total RNASeq lit for Small RNA Libraries (Applied Biosystems now part of Thermo Fisher Scientific) according to the manufacturer's instructions. Purification was performed on 10\% TBE-Urea gels stained with Sybr Gold nucleic acid gel stain (both from Invitrogen now part of Thermo Fisher Scientific). Final purification was performed using PureLink PCR Micro Kit (Invitrogen). Final libraries were quality checked using High Sense DNA kit on Bioanalyzer (all from Agilent, Santa Clara, CA). Concentration of each library was determined using the SOLID Library TaqMan Quantitation Kit (Life Technologies now part of Thermo Fisher Scientific). Each library was clonally amplified on SOLiD P1 DNA Beads by emulsion PCR (ePCR). Emulsions were broken with butanol, and ePCR beads enriched for template-positive beads by hybridization with magnetic enrichment beads. Template-enriched beads were extended at the $3^{\prime}$ end in the presence of terminal transferase and $3^{\prime}$ bead linker. Beads with the clonally amplified DNA were deposited onto SOLiD sequencing slide and sequenced on SOLiD 5500 Instrument using the 50-base sequencing chemistry.

Bioinformatic Analysis Raw data quality assessment, read trimming read mapping and miRNA expression profiling were carried out in CLC Genomics Workbench tool version 8.0.2 (CLC Bio now part of Qiagen, Venlo, Netherlands) using annotated Mus musculus miRNA sequences according to the miRBase release 21 as a mapping reference.

\section{In vitro Experiments \\ Cell Cultures}

$6 \times 10^{4}$ cell $/ \mathrm{ml}$ passage 2 MSCs were plated in cell culture dishes $\left(1.5 \times 10^{4} / \mathrm{cm}^{2}\right)$. After $24 \mathrm{~h}$ incubation, MSC cultures were exposed to B16F1-derived exosomes $(40 \mu \mathrm{g} / \mathrm{ml}$ exosomal proteins; $1.5 \times 10^{11}$ exosomes) at every $24 \mathrm{~h}$. Samples were exposed to exosomes for $24,48,72$, and $96 \mathrm{~h}$ and then harvested in method-competent buffers.

\section{Visualization of Labeled Exosome Internalization in MSCs}

To examine the uptake of exosomes by MSCs, cells were plated to black 24-well Visiplates $\left(1 \times 10^{4}\right.$ cells/well $)$ and incubated for $24 \mathrm{~h}$. The exosomes were labeled with Dil dye $\left(1,1^{\prime}\right.$-dioctadecyl$3,3,3^{\prime}, 3^{\prime}$-tetramethylindocarbocyanine perchlorate, PromoKine, Heidelberg, Germany) and the MSC cultures were labeled with $\mathrm{DiO}$ dye $\left(3,3^{\prime}\right.$-dioctadecyloxacarbo-cyanine perchlorate, PromoKine) according to the manufacturer's instructions. Dillabeled exosomes were washed in DPBS by ultracentrifugation (at $150,000 \mathrm{~g}$ for $1 \mathrm{~h}$ at $4^{\circ} \mathrm{C}$ ). Forty micrograms per milliliter DiL-labeled exosomes were added to DiO-labeled MSC cultures and the exosome uptake was followed for $24 \mathrm{~h}$ in the Celldiscoverer 7 automated live cell imaging system (Zeiss, Oberkochen, Germany). After $24 \mathrm{~h}$, the cells were fixed with $4 \%$ paraformaldehyde solution and a nucleus staining was performed 
using DAPI (Life Technologies now part of Thermo Fisher Scientific). Then, 5 image z-stacks were acquired for both channels by Operetta High Content Screening System (Perkin Elmer, Waltham, MA). The stacks were maximum intensity projected and then analyzed automatically using a customized version of CellProfiler (18). Nuclei were detected with Otsuadaptive threshold combined with diameter based filtering, then cytoplasms were identified with propagation method seeded from the nuclei and using the exosome channel. Exosomes were located with a customized version of A-trous wavelet transform based spot detection (19). Several wavelet levels were used to ensure the detection of exosomes with various size and then the overlaps were removed based on circularity measures. Finally, the exosome numbers per cell were identified using MATLAB programming, the diagrams were created in Microsoft Excel.

\section{Cell Proliferation}

After $72 \mathrm{~h}$ incubation, exosome-exposed and control MSC cultures were dissociated with trypsin from the culture surface. Cells were washed in medium and counted in a Bürker chamber and a cell counter (Bio-Rad, TC10 Automated Cell Counter).

\section{Detection of Apoptosis}

Exosome-exposed MSCs and control cells were treated with $100 \mathrm{ng} / \mathrm{ml}$ mouse TNF $\alpha$ (R\&D Systems). After $24 \mathrm{~h}$ incubation, cell death was determined by the Annexin V Apoptosis Detection Kit with PI (Biolegend, San Diego, CA) according to the manufacturer's recommendations. Samples were measured by FACS Calibur flow cytometer (BD Biosciences), data were analyzed by Flowing Software (Cell Imaging Core, Turku Center for Biotechnology, Finland) where percent of positive cells was determined by relative fluorescence intensity and the results were expressed as mean of percentage of positive cells (\%) \pm SD. Cells that are annexin-V/PI double positive show the sign of late apoptosis, while cells that are annexin- $\mathrm{V}$ positive and $\mathrm{PI}$ negative indicate early apoptosis. Annexin-V negative and PI positive cells are necrotic, viable cells are both annexin- $\mathrm{V}$, and propidium iodide (PI) negative.

\section{RNA Preparation, Melan-A, and Mitf Quantitative Real-Time PCR (QRT-PCR)}

Total RNA of biological samples was purified using the QuickRNA MiniPrep isolation kit of \#R1054S (Zymo Research Irvine, CA). All the preparation steps were carried out according the manufacturer's instructions. RNA samples were stored at $-80^{\circ} \mathrm{C}$ in the presence $30 \mathrm{U}$ of Prime RNAse inhibitor (Fermentas, part of Thermo Fisher Scientific) for further analysis. The quantity of isolated RNA samples was checked by spectrophotometry (NanoDrop 3.1.0, Rockland, DE).

QRT-PCR was performed on a RotorGene 3000 instrument (Corbett Research, Sydney, Australia) with gene-specific primers and SybrGreen protocol to monitor gene expression. One microgram of total RNA was reverse transcribed with random primers using the High-Capacity cDNA Archive Kit (Applied Biosystems) according to the manufacturer's instructions in final volume of $30 \mu \mathrm{l}$. The temperature profile of the reverse transcription was the following: $10 \mathrm{~min}$ at room temperature, $2 \mathrm{~h}$ at $37^{\circ} \mathrm{C}, 5 \mathrm{~min}$ on ice, and finally $10 \mathrm{~min}$ at $75^{\circ} \mathrm{C}$ for enzyme inactivation. These steps were carried out in a Thermal Cycler machine (MJ Research, Marshall Scientific, Hampton, NH). After dilution with $30 \mu \mathrm{l}$ of water, $1 \mu \mathrm{l}$ of the diluted reaction mix was used as template in the QRT-PCR. Reactions were done with FastStart SYBR Green Master mix (Roche) according to the manufacturer's instructions at a final primer concentration of $250 \mathrm{nM}$ under the following conditions: $15 \mathrm{~min}$ at $95^{\circ} \mathrm{C}, 40$ cycles of $95^{\circ} \mathrm{C}$ for $15 \mathrm{~s}, 60^{\circ} \mathrm{C}$ for $25 \mathrm{~s}$, and $72^{\circ} \mathrm{C}$ for $25 \mathrm{~s}$. The fluorescence intensity of SybrGreen dye was detected after each amplification step. Melting temperature analysis was done after each reaction to check the quality of the products. Primers were designed using the online Roche Universal Probe Library Assay Design Center. The quality of the primers was verified by MS analysis provided by Bioneer (Daejeon, Republic of Korea). Relative expression ratios were calculated as normalized ratios to MmRpl27 (Mus musculus ribosomal protein L27) gene. Nontemplate control sample was used for each PCR run to check the primer-dimer formation. The final relative gene expression ratios were calculated as delta-delta $\mathrm{Ct}$ values. Information about the genes and the primers is collected in Table S1.

\section{RNA Isolation, Reverse Transcription, and TaqMan Panel}

RNAs were isolated from MSC cells with Qiagen RNeasy mini kit (Qiagen) based on the manufacturer's instruction. RNA concentrations were measured by NanoDrop spectrophotometer (NanoDrop). cDNAs were reverse transcribed with TaqMan Reverse Transcription Reagents (Thermo Fisher Scientific) following the manufacturer's instruction. For the TaqMan QRTPCR panel, cDNA mixture of three parallel samples was used in case of each condition: control, 6, 24, and $72 \mathrm{~h}$ exosome treatment applied on MSC cells derived from four mice. Eighty nanogram cDNA and TaqMan Gene Expression Master Mix (Thermo Fisher Scientific) were used for the qPCR experiment. Expression of the examined 44 genes was calculated by $\Delta \Delta \mathrm{Ct}$ method and normalized to the average $\mathrm{Ct}$ values of 4 internal controls (PPIA, 18S RNA, ACTB, and GAPDH).

\section{PD-1 Detection}

\section{PD-1 detection by Western blotting}

The cells were washed three times in PBS, then were lysed in TENT Buffer $(50 \mathrm{mM}$ Tris-HCl, $2 \mathrm{mM}$ EDTA, $150 \mathrm{mM}$ $\mathrm{NaCl}, 1 \%$ TritonX-100, completed with $1 \mathrm{x}$ protease inhibitor cocktail (Roche). Protein samples were separated and blotted as described above.

For PD-1 protein level detection, anti-PD-1 (clone: RMO114, Biolegend) antibody was used in 1:1,000 dilution. Anti-rat horseradish peroxidase (HRP)-conjugated secondary antibody (R\&D Systems) was used in 1:500 dilution.

\section{PD-1 detection by Immunofluorescence microscopy}

MSC cultures were fixed with $4 \%$ paraformaldehyde for $10 \mathrm{~min}$, and blocked in PBS buffer containing 5\% BSA (Sigma-Aldrich) for $60 \mathrm{~min}$ at room temperature. After blocking, cells were incubated with primary antibody in DPBS buffer containing 
1.2\% BSA overnight at $4^{\circ} \mathrm{C}$. As primary antibodies, 1:200 antiPD-1 (clone: RMO1-14, Biolegend,) and 1:200 anti-MLANA (Byorbit) were used. After incubation, cells were washed three times in PBS, and incubated for $1 \mathrm{~h}$ at room temperature with 1:100 Alexa Fluor 647-conjugated anti-rat antibody (Jackson ImmunoResearch Laboratories, Baltimore, PA) or 1:500 Alexa Fluor 555-conjugated anti-rabbit antibody (Thermo Fisher Scientific). Tubulin was stained with 1:500 Alexa Fluor 488conjugated anti-tubulin- $\alpha$ antibody (clone: 10D8, Biolegend). Nucleus staining was performed using DAPI (Life Technologies). Slides were washed three times with DPBS between each step. Images were obtained at 60x magnification using an Olympus confocal laser scanning microscope.

\section{PD-1 detection by STORM super-resolution imaging}

All dSTORM super-resolution experiments were performed on a custom-made inverted microscope based on a Nikon Eclipse Ti-E frame. After being conditioned (through spatial filtering via fiber coupling and beam expansion) the applied laser beams were focused into the back focal plane of the microscope objective (Nikon CFI Apo 100x, NA = 1.49), which produced a collimated beam on the sample. The angle of illumination was set via a tilting mirror mounted into a motorized gimbal holder and placed into the conjugate plane of the sample. All the dSTORM images were captured under EPI illumination at an excitation wavelength of $647 \mathrm{~nm}$ (Nikon: $647 \mathrm{~nm}, 300 \mathrm{~mW}$ ). The laser power, controlled via an acousto-optic tunable filter (AOTF), was set to $4 \mathrm{~kW} / \mathrm{cm}^{2}$ on the sample plane. An additional laser (Nichia, Anan, Tokushima, Japan, $405 \mathrm{~nm}, 60 \mathrm{~mW}$ ) was used for both reactivation and reference measurements. Images were captured by an Andor iXon3 897 BV EMCCD digital camera $(512 \times 512$ pixels with pixel size of $16 \mu \mathrm{m}$ ). The size of the illuminated region of the sample was matched to the size of the detector, which determined the field of view $\left(\mathrm{FOV}=80 \times 80 \mu \mathrm{m}^{2}\right)$. Frame stacks for dSTORM super-resolution imaging were typically captured at a reduced image size (crop mode), when only the central $128 \times 128$ pixel region was selected. A fluorescence filter set (Semrock, Rochester, NY, LF405/488/561/635-A-000) was used to select and separate the excitation and emission lights in the microscope. Additional emission filters (Semrock, BLP01647R-25) were used in the detection path to further clean the fluorescent light spectrally for the reduction of spectral crosstalk.

During the measurements, the perfect focus system of the microscope was used to keep the sample in focus with a precision of $<30 \mathrm{~nm}$. The storage buffer on the sample was replaced with a special switching buffer (20). Typically, 10,000 frames were captured with an exposure time of $30 \mathrm{~ms}$. Reference images with full size FOV were captured at low intensity when the majority of fluorescent molecules were still active and the overall structure of the labeled sample could be visualized.

The captured and stored image stacks were evaluated and analyzed by rainSTORM localization software (21). The individual images of single molecules were fitted with a Gaussian point spread function and their center positions were associated with the position of the fluorescent molecule. Localizations were filtered via their intensity, ellipticity and standard deviation values. Localizations with precisions of $<45 \mathrm{~nm}$ were only used to form the final image. The estimated mean precision of the accepted localizations was $19 \mathrm{~nm}$. Mechanical drift introduced by either the mechanical movement of the sample or thermal effects was analyzed and reduced by means of a blind drift correction algorithm. Spatial coordinates of the localized molecules were stored and the final super-resolved image was visualized. The multicolor merged images were generated by Image J software.

\section{In vivo Experiments Mouse Model}

B16F1 melanoma cells were administrated intravenously $\left(1 \times 10^{5}\right.$ cell/100 $\mu \mathrm{l}$ ) to $6-8$ week old female $\mathrm{C} 57 \mathrm{BL} / 6 \mathrm{~N}$ mice (Charles River Laboratories). One week later, tumor bearing mice were randomized and divided into 3 groups $(n=10$ /group). Mice were injected intravenously with control buffer $(100 \mu \mathrm{l})$, exosome exposed MSCs $\left(1 \times 10^{5}\right.$ cell/100 $\left.\mu \mathrm{l}\right)$ or exosomes $(40 \mu \mathrm{g} / 100$ $\mu$ l) 7, 8, 9, 10, 11 days after the tumor injection (Table S2). One week after the first MSC administration, 3 animals/group were euthanized, their lungs were removed, photographed and stored at $-80^{\circ} \mathrm{C}$ for further protein, mRNA and histological analyses. The remained mice were observed for 10 more days. At the end point, the animals were euthanized, and the tumor metastases were investigated not only in their lung, but also in their entire body and removed for histological analysis. Experiments were repeated 3 times. All animal experiments were performed in accordance with national (1998. XXVIII; 40/2013) and European (2010/63/EU) animal ethics guidelines. The experimental protocols were approved by the Animal Experimentation and Ethics Committee of the Biological Research Centre of the Hungarian Academy of Sciences and the Hungarian National Animal Experimentation and Ethics Board (clearance number: XVI./03521/2011 and XV./78/2018).

\section{Tumor Coverage}

Tumor coverage of lungs was determined by the analysis of acquired images using the ImageJ software. The area of tumors and the healthy regions was measured and the mean percentage, $\mathrm{SD}$, and $p$ values were calculated in Microsoft Excel.

\section{Quantification of Metastases Associated Vessel Diameters}

From native animal lungs $4 \mu \mathrm{m}$ criostat sections were made on silanized slides, than fixed and retrieved by Fix and Perm A-B solution (Thermo Fisher Scientific, USA) for $20 \mathrm{~min}$. Sections were counterstained by conventional hematoxilin for $30 \mathrm{~min}$ than washed in tap water and coverslipped. Sections were digitalized by automatic slide-scanner (3DHistech, Hungary), using the software 3DHISTECH Pannoramic Viewer (3DHistech, Hungary). Strict tumor border was carefully marked then vessel diameters were measured.

\section{Cytokine and Chemokine Array}

Lung samples were lysed in NP40 cell lysis buffer (Thermo Fisher Scientific, USA) and protein content was measured by the Pierce BCA Protein Assay kit (Thermo Fisher Scientific, USA). Expression levels of different cytokines in pooled lung specimens were determined using Mouse Cytokine Array Panel A (R\&D 
Systems, Cat. no. ARY006), according to the manufacturer's instructions. Immunoreactive signals were detected by using LICOR ODYSSEY Fc (Dual-mode imaging system) imager followed by analysis with Image Studio Lite v5.2.

\section{Custom TaqMan Array Panels}

QRT-PCR was performed on an ABI Prism 7000 sequence detection system (Applied Biosystems) using specific predesigned customized 96-well TaqMan Array oncogene panels (Thermo Fisher Scientific) containing 44 specifically selected primers and probes according to extensive literature work. Total RNA was isolated using TRIzol (Thermo Fisher Scientific), DNase treatment was performed according to the manufacturer's protocol, and then total RNA was transcribed into cDNA using High Capacity cDNA Kit (Thermo Fisher Scientific). PCR amplification was performed using TaqMan primers and probes and thermal cycle conditions were set as follows: $2 \mathrm{~min}$ at $50^{\circ} \mathrm{C}, 10 \mathrm{~min}$ at $95^{\circ} \mathrm{C}$, and 40 cycles of $15 \mathrm{~s}$ at $95^{\circ} \mathrm{C}$ and $1 \mathrm{~min}$ at $60^{\circ} \mathrm{C}$. As internal control transcripts of ACTB (Assay ID: Mm00607939_s1), GAPDH (Assay ID: Mm99999915_g1), and PPIA (Assay ID: Mm02342430_g1) were determined. The amount of the transcripts was normalized to those of the housekeeping genes using the $\Delta \mathrm{Ct}$ method. Finally the results were normalized to the expression of the vehicle control ( $\Delta \Delta \mathrm{Ct}$ method).
For relationship discovery Hierarchical Cluster Analysis was performed by $\mathrm{R}$ software. In detail, the "bottom up" agglomerative hierarchical clustering strategy was used and results represented in a tree-based dendrogram [For refer the $\mathrm{R}$ software: (22)].

\section{Network Representation by Ingenuity Pathway Analysis}

According to literature data, we established a protein network from the in vivo overexpressed genes. A custom graphical representation of this network was generated using the Path Explorer tool of the IPA Path Designer. Genes are represented as red nodes, using various shapes that represent the functional class of the gene product. In a few cases proteins are substituted with the complex, which they are involved in. To identify the potential exosomal factors, which can induce the activation of the network, a list was generated from the exosomal proteins detected by LC-MS/MS and the exosomal miRNAs identified by SOLiD sequencing. The Grow tool in the IPA Path Designer revealed significant interactions between the network and the generated list (the interacting exosomal factors are listed in gray boxes and the types of relationships are indicated in parentheses). During construction in the IPA, the significance level was set to "experimentally observed" data to avoid the representation of predicted, unproven interactions.
A

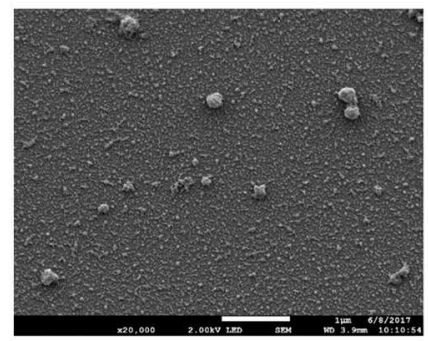

C

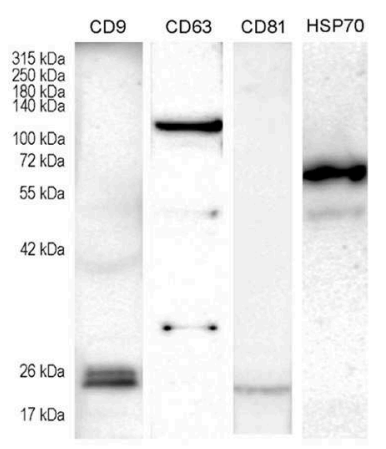

B
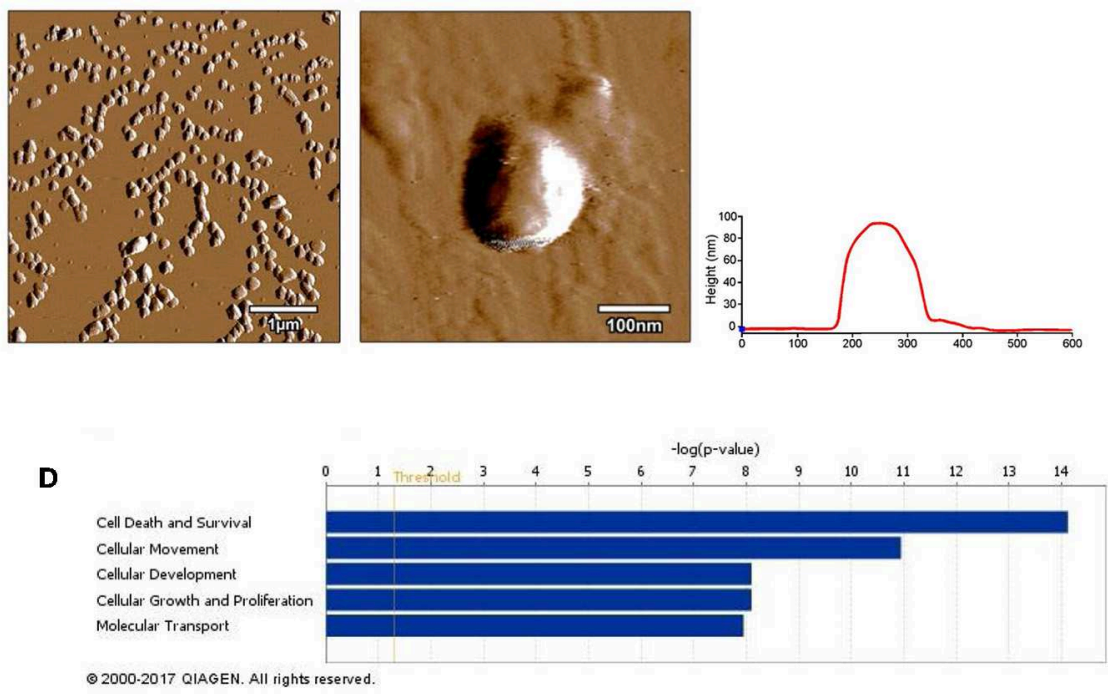

E

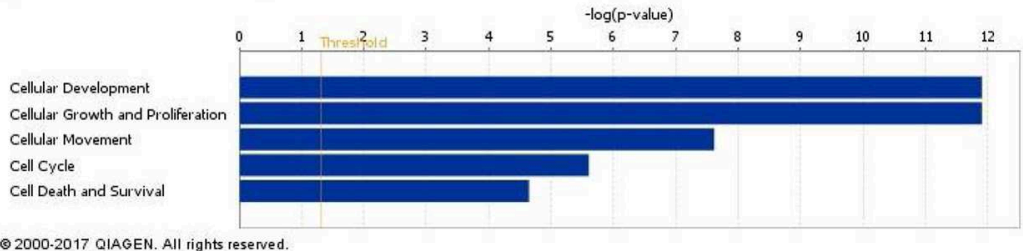

FIGURE 1 | Characterization of B16F1 melanoma cell culture-derived exosomes. (A) Scanning electron micrograph of melanoma exosomes. (B) Atomic force microscopy images of exosomes. Left and middle pictures represent the shape and surface topography of vesicles, right graph represents height profile. (C) Western blot analysis of common exosome markers (CD9, CD63, CD81, and HSP70). (D,E) Top 5 molecular and cellular functions identified by Ingenuity Pathway Analysis of exosomal proteins and miRNAs. 
TABLE 1 | List of exosomal proteins identified by LC-MS/MS.

\begin{tabular}{|c|c|c|}
\hline ID & Symbol & Entrez gene name \\
\hline P97857 & ADAMTS1 & $\begin{array}{l}\text { ADAM metallopeptidase with } \\
\text { thrombospondin type } 1 \text { motif } 1\end{array}$ \\
\hline Q3TNX8 & ADAMTS4 & $\begin{array}{l}\text { ADAM metallopeptidase with } \\
\text { thrombospondin type } 1 \text { motif } 4\end{array}$ \\
\hline Q640N1 & AEBP1 & AE binding protein 1 \\
\hline P05064 & ALDOA & Aldolase, fructose-bisphosphate A \\
\hline P97429 & ANXA4 & Annexin A4 \\
\hline P48036 & ANXA5 & Annexin A5 \\
\hline P08226 & APOE & Apolipoprotein E \\
\hline Q3TWT5 & ASAH1 & $\mathrm{N}$-acylsphingosine amidohydrolase 1 \\
\hline Q3TXF9 & ATP1A1 & ATPase $\mathrm{Na}^{+} / \mathrm{K}^{+}$transporting subunit alpha 1 \\
\hline P97370 & ATP1B3 & ATPase $\mathrm{Na}^{+} / \mathrm{K}^{+}$transporting subunit beta 3 \\
\hline Q1XID4 & ATP6AP2 & ATPase $\mathrm{H}^{+}$transporting accessory protein 2 \\
\hline Q9JL18 & BACE2 & Beta-site APP-cleaving enzyme 2 \\
\hline O55107 & $B S G$ & Basigin (Ok blood group) \\
\hline Q8R2Q8 & Bst2 & Bone marrow stromal cell antigen 2 \\
\hline Q9WVT6 & CA14 & Carbonic anhydrase 14 \\
\hline P41731 & CD63 & CD63 molecule \\
\hline P35762 & CD81 & CD81 molecule \\
\hline P10605 & CTSB & Cathepsin B \\
\hline P18242 & CTSD & Cathepsin D \\
\hline P29812 & DCT & Dopachrome tautomerase \\
\hline P57776 & EEF1D & $\begin{array}{l}\text { Eukaryotic translation elongation factor } 1 \\
\text { delta }\end{array}$ \\
\hline Q3UAM9 & ENG & Endoglin \\
\hline P17182 & ENO1 & Enolase 1 \\
\hline P19096 & FASN & Fatty acid synthase \\
\hline P30416 & FKBP4 & FK506 binding protein 4 \\
\hline P11276 & FN1 & Fibronectin 1 \\
\hline P09528 & $\mathrm{FTH} 1$ & Ferritin heavy chain 1 \\
\hline P16858 & GAPDH & Glyceraldehyde-3-phosphate dehydrogenase \\
\hline P08752 & GNAI2 & G protein subunit alpha i2 \\
\hline Q3TAV1 & GPNMB & Glycoprotein nmb \\
\hline P19157 & GSTP1 & Glutathione S-transferase pi 1 \\
\hline P11499 & HSP90AB1 & $\begin{array}{l}\text { Heat shock protein } 90 \text { alpha family class } B \\
\text { member } 1\end{array}$ \\
\hline B1B0C7 & HSPG2 & Heparan sulfate proteoglycan 2 \\
\hline Q9CQW9 & IFITM3 & Interferon induced transmembrane protein 3 \\
\hline G3UYZ1 & IGSF8 & Immunoglobulin superfamily member 8 \\
\hline Q91VK4 & ITM2C & Integral membrane protein $2 \mathrm{C}$ \\
\hline P02468 & LAMC1 & Laminin subunit gamma 1 \\
\hline Q60961 & LAPTM4A & Lysosomal protein transmembrane 4 alpha \\
\hline P35951 & LDLR & Low density lipoprotein receptor \\
\hline Q07797 & LGALS3BP & Galectin 3 binding protein \\
\hline Q3U2W5 & LGALS8 & Galectin 8 \\
\hline P16056 & MET & $\begin{array}{l}\text { MET proto-oncogene, receptor tyrosine } \\
\text { kinase }\end{array}$ \\
\hline P21956 & MFGE8 & Milk fat globule-EGF factor 8 protein \\
\hline Q2TA50 & MLANA & Melan-A \\
\hline Q6NVG5 & MREG & Melanoregulin \\
\hline Q9EPX2 & PAPLN & $\begin{array}{l}\text { Papilin, proteoglycan like sulfated } \\
\text { glycoprotein }\end{array}$ \\
\hline
\end{tabular}

(Continued)
TABLE 1 | Continued

\begin{tabular}{|c|c|c|}
\hline ID & Symbol & Entrez gene name \\
\hline Q3UIP2 & PCOLCE & Procollagen C-endopeptidase enhancer \\
\hline Q811J2 & LOC72520 & LOC72520 protein \\
\hline Q80Y09 & PDCD6IP & Programmed cell death 6 interacting protein \\
\hline P62962 & PFN1 & Profilin 1 \\
\hline P09411 & PGK1 & Phosphoglycerate kinase 1 \\
\hline P52480 & PKM & Pyruvate kinase, muscle \\
\hline Q9CZB2 & PMEL & Premelanosome protein \\
\hline P17742 & PPIA & Peptidylprolyl isomerase A \\
\hline P35700 & PRDX1 & Peroxiredoxin 1 \\
\hline Q61171 & PRDX2 & Peroxiredoxin 2 \\
\hline Q543S0 & PRELP & $\begin{array}{l}\text { Proline and arginine rich end leucine rich } \\
\text { repeat protein }\end{array}$ \\
\hline P53994 & RAB2A & RAB2A, member RAS oncogene family \\
\hline Q8CCG5 & RALB & RAS like proto-oncogene B \\
\hline O89086 & RBM3 & RNA binding motif (RNP1, RRM) protein 3 \\
\hline P35980 & RPL18 & Ribosomal protein L18 \\
\hline Q3U5P4 & SCPEP1 & Serine carboxypeptidase 1 \\
\hline O08992 & SDCBP & Syndecan binding protein \\
\hline QOVGP2 & SEMA3B & Semaphorin 3B \\
\hline P32261 & SERPINC1 & Serpin family $\mathrm{C}$ member 1 \\
\hline P10852 & SLC3A2 & Solute carrier family 3 member 2 \\
\hline Q3UQM7 & SLC7A5 & Solute carrier family 7 member 5 \\
\hline 009044 & SNAP23 & Synaptosome associated protein 23 \\
\hline Q64337 & SQSTM1 & Sequestosome 1 \\
\hline Q8Cl59 & STEAP3 & STEAP3 metalloreductase \\
\hline Q3TDG9 & STX12 & Syntaxin 12 \\
\hline 070439 & STX7 & Syntaxin 7 \\
\hline P40749 & SYT4 & Synaptotagmin 4 \\
\hline O88968 & TCN2 & Transcobalamin 2 \\
\hline Q542D9 & TFRC & Transferrin receptor \\
\hline P39876 & TIMP3 & TIMP metallopeptidase inhibitor 3 \\
\hline Q4FJX7 & TINAGL1 & Tubulointerstitial nephritis antigen like 1 \\
\hline Q9DCS1 & TMEM176A & Transmembrane protein $176 \mathrm{~A}$ \\
\hline Q9R1Q6 & TMEM176B & Transmembrane protein 176B \\
\hline Q9CZX7 & TMEM55A & Transmembrane protein 55A \\
\hline Q9QY73 & TMEM59 & Transmembrane protein 59 \\
\hline O88746 & TOM1 & Target of myb1 membrane trafficking protein \\
\hline O89023 & TPP1 & tripeptidyl peptidase 1 \\
\hline Q3UCW0 & TSG101 & Tumor susceptibility 101 \\
\hline Q4FJW7 & TSPAN4 & Tetraspanin 4 \\
\hline Q8BJU2 & TSPAN9 & Tetraspanin 9 \\
\hline P11344 & TYR & Tyrosinase \\
\hline P07147 & TYRP1 & Tyrosinase related protein 1 \\
\hline O70404 & VAMP8 & Vesicle associated membrane protein 8 \\
\hline Q8R0J7 & VPS37B & VPS37B, ESCRT-I subunit \\
\hline Q8R105 & VPS37C & VPS37C, ESCRT-I subunit \\
\hline O88384 & VTI1B & $\begin{array}{l}\text { Vesicle transport through interaction with } \\
\text { t-SNAREs } 1 B\end{array}$ \\
\hline A8DUQ1 & HBBT1 & Beta-globin \\
\hline P70356 & MELA & Gag-pol poliprotein \\
\hline P70355 & MELA & Envelope protein \\
\hline
\end{tabular}




\section{Statistical Analysis}

All of the data are presented as the mean \pm SD or SEM and represent minimum of three independent experiments. Statistical parameters including statistical analysis, statistical significance, and $\mathrm{n}$ value are reported in the figure legends. For in vivo experiments $n=$ number of animals. For statistical comparison, we performed two-tailed Student's $t$-test. A value of $p<0.05$ was considered significant [represented as ${ }^{*} p<0.05$, not significant (n.s.)].

\section{RESULTS}

\section{Isolated Vesicles Show Exosomal Properties}

First, we isolated extracellular vesicles from B16F1 mouse melanoma cells. As it was shown by SEM and AFM (Figures 1A,B), the isolated fraction indeed contained exosomes as the particles were cap-shaped, and their size was within the 40-120 nm range. Then, presence of molecules (CD9, CD63, CD81, and HSP70), characteristic for exosomes (4, 17) was assessed by Western blotting (Figure 1C).

Exosomes were then subjected to large-scale analysis to determine their protein and miRNA profiles. Whole proteome analysis (using LC-MS/MS) and bioinformatics tools (used to identify elements of the exosome's proteomics spectrum in the UniProt database, and to compare the findings with entries of the ExoCarta database) revealed that 95 distinct proteins were identified in melanoma exosomes (Table 1). These proteins exhibited $86.3 \%$ overlap with molecules listed as characteristic exosome protein markers in ExoCarta. Further, miRNA sequencing (using SOLiD 5500xl technology) identified 168 known miRNA elements (Table 2) which, similarly to the proteomics data, exhibited a large (93.5\%) overlap with molecules listed as characteristic exosome miRNA markers in ExoCarta.

To uncover the functional significance of the proteomics and miRNA sequencing data, IPA was employed. This analysis has shown that the identified proteins most probably participated in cellular and molecular processes such as "Cell Death and Survival," "Cellular Movement," "Cell-to-Cell Signaling and Interaction," "Cellular Growth and Proliferation," and "Cell Morphology" ( $P_{\text {range }}=7.53 \times 10^{-15}-9.32 \times 10^{-4}$ significance range) (Figure 1D). Very similarly to these data, functions of the identified miRNAs were suggested to be linked to mechanisms of "Cellular Development," "Cellular Growth and Proliferation," "Cellular Movement," "Cell Cycle," and "Cell Death and Proliferation" $\left(P_{\text {range }}=1.25 \times 10^{-12}-4.88 \times 10^{-2}\right.$ significance range) (Figure 1E).

\section{Tumor Exosome Exposure Resulted in Oncogenic Reprogramming of MSCs in vitro}

Then, we investigated the effect of exosomes on biological processes (e.g., proliferation, survival, malignant transformation, etc.) of MSCs, which are generally considered as proper in vitro models of tumor stroma (12). For these experiments, MSC
TABLE 2 | List of exosomal miRNAs identified by SOLiD 5500xl technology.

\begin{tabular}{|c|c|c|}
\hline Symbol & Seed regio & ID \\
\hline \multirow[t]{4}{*}{ let-7a-3p } & UAUACAA & mmu-let-7a-1-3p \\
\hline & & mmu-let-7b-3p \\
\hline & & mmu-let-7c-2-3p \\
\hline & & mmu-let-7f-1-3p \\
\hline \multirow[t]{8}{*}{ let-7a-5p } & GAGGUAG & mmu-let-7a-5p \\
\hline & & mmu-let-7b-5p \\
\hline & & mmu-let-7c-5p \\
\hline & & mmu-let-7d-5p \\
\hline & & mmu-let-7e-5p \\
\hline & & mmu-let-7f-5p \\
\hline & & mmu-let-7g-5p \\
\hline & & mmu-mir-98-5p \\
\hline let-7d-3p & UAUACGA & mmu-let-7d-3p \\
\hline let-7i-3p & UGCGCAA & mmu-let-7i-3p \\
\hline \multirow[t]{2}{*}{$m i R-100-5 p$} & ACCCGUA & mmu-mir-99a-5p \\
\hline & & mmu-mir-99b-5p \\
\hline miR-101-3p & ACAGUAC & mmu-mir-101a-3p \\
\hline miR-103-1-5p & GCUUCUU & mmu-mir-107-5p \\
\hline \multirow[t]{2}{*}{ miR-103-3p } & GCAGCAU & mmu-mir-103-3p \\
\hline & & mmu-mir-107-3p \\
\hline \multirow[t]{2}{*}{ miR-10a-5p } & ACCCUGU & mmu-mir-10a-5p \\
\hline & & mmu-mir-10b-5p \\
\hline miR-1191a & AGUCUUA & mmu-mir-1191a \\
\hline miR-1249-3p & CGCCCUU & mmu-mir-1249-3p \\
\hline \multirow[t]{3}{*}{$m i R-125 b-5 p$} & CCCUGAG & mmu-mir-125a-5p \\
\hline & & mmu-mir-125b-5p \\
\hline & & mmu-mir-351-5p \\
\hline miR-126a-5p & 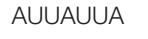 & mmu-mir-126a-5p \\
\hline miR-128-3p & CACAGUG & mmu-mir-128-3p \\
\hline \multirow[t]{2}{*}{ miR-129-1-3p } & AGCCCUU & mmu-mir-129-1-3p \\
\hline & & mmu-mir-129-2-3p \\
\hline miR-129b-5p & CUUUUUG & mmu-mir-129b-5p \\
\hline \multirow[t]{4}{*}{ miR-130a-3p } & AGUGCAA & mmu-mir-130a-3p \\
\hline & & mmu-mir-130b-3p \\
\hline & & mmu-mir-301a-3p \\
\hline & & mmu-mir-301b-3p \\
\hline miR-130a-5p & CUCUUUU & mmu-mir-130a-5p \\
\hline$m i R-130 b-5 p$ & CUCUUUC & mmu-mir-130b-5p \\
\hline miR-132-3p & AACAGUC & mmu-mir-132-3p \\
\hline$m i R-132-5 p$ & ACCGUGG & mmu-mir-132-5p \\
\hline miR-135a-5p & AUGGCUU & mmu-mir-135a-5p \\
\hline$m i R-138-5 p$ & GCUGGUG & mmu-mir-138-5p \\
\hline$m i R-139-5 p$ & CUACAGU & mmu-mir-139-5p \\
\hline$m i R-140-3 p$ & ACCACAG & mmu-mir-140-3p \\
\hline$m i R-140-5 p$ & AGUGGUU & mmu-mir-140-5p \\
\hline miR-142-3p & GUAGUGU & mmu-mir-142a-3p \\
\hline$m i R-143-5 p$ & & $m m u-m i r-143-5 p$ \\
\hline$m i R-144-3 p$ & ACAGUAU & mmu-mir-144-3p \\
\hline$m i R-144-5 p$ & GAUAUCA & mmu-mir-144-5p \\
\hline$m i R-145-5 p$ & UCCAGUU & mmu-mir-145a-5p \\
\hline
\end{tabular}


TABLE 2 | Continued

\begin{tabular}{|c|c|c|}
\hline Symbol & Seed regio & ID \\
\hline miR-146a-5p & GAGAACU & mmu-mir-146a-5p \\
\hline miR-148a-3p & CAGUGCA & mmu-mir-148b-3p \\
\hline miR-151-3p & UAGACUG & mmu-mir-151-3p \\
\hline miR-15a-3p & AGGCCAU & mmu-mir-15a-3p \\
\hline miR-15b-3p & GAAUCAU & mmu-mir-15b-3p \\
\hline miR-16-2-3p & CCAAUAU & mmu-mir-16-2-3p \\
\hline \multirow[t]{6}{*}{$m i R-16-5 p$} & AGCAGCA & mmu-mir-15a-5p \\
\hline & & mmu-mir-15b-5p \\
\hline & & mmu-mir-16-5p \\
\hline & & mmu-mir-195a-5p \\
\hline & & mmu-mir-322-5p \\
\hline & & mmu-mir-497a-5p \\
\hline miR-17-3p & CUGCAGU & mmu-mir-17-3p \\
\hline \multirow[t]{4}{*}{$m i R-17-5 p$} & AAAGUGC & mmu-mir-106b-5p \\
\hline & & mmu-mir-17-5p \\
\hline & & mmu-mir-20a-5p \\
\hline & & mmu-mir-93-5p \\
\hline$m i R-181 a-1-3 p$ & CCAUCGA & mmu-mir-181a-1-3p \\
\hline \multirow[t]{4}{*}{ miR-181a-5p } & ACAUUCA & mmu-mir-181a-5p \\
\hline & & mmu-mir-181b-5p \\
\hline & & mmu-mir-181c-5p \\
\hline & & mmu-mir-181d-5p \\
\hline miR-1827 & GAGGCAG & mmu-mir-709 \\
\hline miR-1839-3p & GACCUAC & mmu-mir-1839-3p \\
\hline miR-185-5p & GGAGAGA & mmu-mir-185-5p \\
\hline$m i R-186-5 p$ & AAAGAAU & mmu-mir-186-5p \\
\hline miR-187-3p & CGUGUCU & mmu-mir-187-3p \\
\hline miR-188-3p & UCCCACA & mmu-mir-188-3p \\
\hline miR-188-5p & AUCCCUU & mmu-mir-188-5p \\
\hline$m i R-18 a-5 p$ & AAGGUGC & mmu-mir-18a-5p \\
\hline$m i R-191-5 p$ & AACGGAA & mmu-mir-191-5p \\
\hline miR-193a-3p & ACUGGCC & mmu-mir-193a-3p \\
\hline miR-1981-3p & AUCUAAC & mmu-mir-1981-3p \\
\hline \multirow[t]{2}{*}{ miR-199a-3p } & CAGUAGU & mmu-mir-199a-3p \\
\hline & & mmu-mir-199b-3p \\
\hline \multirow[t]{2}{*}{ miR-199a-5p } & CCAGUGU & mmu-mir-199a-5p \\
\hline & & mmu-mir-199b-5p \\
\hline \multirow[t]{2}{*}{ miR-19b-3p } & GUGCAAA & mmu-mir-19a-3p \\
\hline & & mmu-mir-19b-3p \\
\hline miR-204-5p & UCCCUUU & $m m u-m i r-211-5 p$ \\
\hline$m i R-21-5 p$ & AGCUUAU & mmu-mir-21a-5p \\
\hline miR-210-3p & UGUGCGU & mmu-mir-210-3p \\
\hline miR-210-5p & GCCACUG & mmu-mir-210-5p \\
\hline miR-219a-5p & GAUUGUC & mmu-mir-219a-5p \\
\hline miR-22-3p & AGCUGCC & mmu-mir-22-3p \\
\hline$m i R-22-5 p$ & GUUCUUC & mmu-mir-22-5p \\
\hline miR-221-3p & GCUACAU & mmu-mir-222-3p \\
\hline miR-223-3p & GUCAGUU & mmu-mir-223-3p \\
\hline miR-224-5p & AAGUCAC & mmu-mir-224-5p \\
\hline \multirow[t]{2}{*}{ miR-23a-3p } & UCACAUU & mmu-mir-23a-3p \\
\hline & & mmu-mir-23b-3p \\
\hline
\end{tabular}

TABLE 2 | Continued

\begin{tabular}{|c|c|c|}
\hline Symbol & Seed regio & ID \\
\hline miR-24-1-5p & UGCCUAC & mmu-mir-24-2-5p \\
\hline miR-24-3p & GGCUCAG & mmu-mir-24-3p \\
\hline miR-26a-5p & UCAAGUA & mmu-mir-26a-5p \\
\hline \multirow[t]{2}{*}{ miR-26a-5p } & UCAAGUA & mmu-mir-26a-5p \\
\hline & & mmu-mir-26b-5p \\
\hline \multirow[t]{2}{*}{ miR-27a-3p } & UCACAGU & mmu-mir-27a-3p \\
\hline & & mmu-mir-27b-3p \\
\hline miR-29a-5p & CUGAUUU & mmu-mir-29a-5p \\
\hline$m i R-29 b-1-5 p$ & CUGGUUU & mmu-mir-29b-1-5p \\
\hline \multirow[t]{3}{*}{ miR-29b-3p } & AGCACCA & mmu-mir-29a-3p \\
\hline & & mmu-mir-29b-3p \\
\hline & & mmu-mir-29c-3p \\
\hline miR-3065-5p & CAACAAA & mmu-mir-3065-5p \\
\hline \multirow[t]{5}{*}{$m i R-30 c-5 p$} & GUAAACA & mmu-mir-30a-5p \\
\hline & & mmu-mir-30b-5p \\
\hline & & mmu-mir-30c-5p \\
\hline & & mmu-mir-30d-5p \\
\hline & & mmu-mir-30e-5p \\
\hline miR-31-3p & GCUAUGC & mmu-mir-31-3p \\
\hline miR-31-5p & GGCAAGA & mmu-mir-31-5p \\
\hline miR-3176 & CUGGCCU & mmu-mir-378d \\
\hline miR-324-5p & GCAUCCC & mmu-mir-324-5p \\
\hline miR-328-3p & UGGCCCU & mmu-mir-328-3p \\
\hline miR-329-3p & ACACACC & mmu-mir-362-3p \\
\hline miR-33-5p & UGCAUUG & mmu-mir-33-5p \\
\hline miR-330-5p & CUCUGGG & mmu-mir-326-3p \\
\hline miR-331-3p & CCCCUGG & mmu-mir-331-3p \\
\hline miR-339-5p & CCCUGUC & mmu-mir-339-5p \\
\hline miR-340-3p & CCGUCUC & mmu-mir-340-3p \\
\hline$m i R-344 a-5 p$ & CAGGCUC & mmu-mir-484 \\
\hline miR-345-5p & CUGACCC & mmu-mir-345-5p \\
\hline \multirow[t]{2}{*}{ miR-3473b } & GGCUGGA & mmu-mir-3473b \\
\hline & & mmu-mir-3473e \\
\hline \multirow[t]{3}{*}{ miR-34a-5p } & GGCAGUG & mmu-mir-34a-5p \\
\hline & & mmu-mir-34b-5p \\
\hline & & mmu-mir-34c-5p \\
\hline miR-34c-3p & AUCACUA & mmu-mir-34b-3p \\
\hline miR-350 & UCACAAA & mmu-mir-350-3p \\
\hline miR-361-5p & UAUCAGA & mmu-mir-361-5p \\
\hline miR-362-5p & AUCCUUG & mmu-mir-362-5p \\
\hline miR-374b-5p & UAUAAUA & mmu-mir-374b-5p \\
\hline \multirow[t]{2}{*}{ miR-378a-3p } & CUGGACU & mmu-mir-378a-3p \\
\hline & & mmu-mir-378c \\
\hline$m i R-378 a-5 p$ & UCCUGAC & mmu-mir-378a-5p \\
\hline miR-3909 & GUCCUCU & mmu-mir-877-3p \\
\hline miR-423-3p & GCUCGGU & mmu-mir-423-3p \\
\hline$m i R-423-5 p$ & GAGGGGC & mmu-mir-423-5p \\
\hline$m i R-425-5 p$ & AUGACAC & mmu-mir-425-5p \\
\hline $\mathrm{miR}-451 \mathrm{a}$ & AACCGUU & mmu-mir-451a \\
\hline miR-501-5p & AUCCUUU & mmu-mir-501-5p \\
\hline miR-503-5p & AGCAGCG & mmu-mir-503-5p \\
\hline
\end{tabular}


TABLE 2 | Continued

\begin{tabular}{lll}
\hline Symbol & Seed regio & ID \\
\hline miR-532-5p & AUGCCUU & mmu-mir-532-5p \\
miR-542-3p & GUGACAG & mmu-mir-542-3p \\
miR-574-5p & GAGUGUG & mmu-mir-574-5p \\
miR-582-5p & UACAGUU & mmu-mir-582-5p \\
miR-652-3p & AUGGCGC & mmu-mir-652-3p \\
miR-670-5p & UCCCUGA & mmu-mir-670-5p \\
miR-700-5p & AAGGCUC & mmu-mir-700-5p \\
miR-744-3p & UGUUGCC & mmu-mir-744-3p \\
miR-744-5p & & mmu-mir-744-5p \\
miR-7a-5p & GGAAGAC & mmu-mir-7a-5p \\
miR-872-3p & GAACUAU & mmu-mir-872-3p \\
miR-872-5p & AGGUUAC & mmu-mir-872-5p \\
miR-9-5p & CUUUGGU & mmu-mir-9-5p \\
miR-92a-3p & AUUGCAC & mmu-mir-25-3p \\
& & mmu-mir-32-5p \\
& & mmu-mir-92a-3p \\
\hline
\end{tabular}

cultures were initiated from mouse abdominal adipose tissue (17) and were subjected to melanoma exosome treatment.

First, we assessed whether exosomes were internalized by MSCs. High-throughput microscopy showed that MSCs [labeled green by the $\mathrm{DiOC}_{18}(3)$ lipid dye] indeed took up exosomes [labeled red by the $\operatorname{DilC}_{18}(3)$ lipid dye] as early as $1-2 \mathrm{~h}$ after application (Figure S1). Importantly, after $24 \mathrm{~h}$, the majority of MSCs were loaded by exosomes (Figures 2A,B). Indeed, image analysis and statistical evaluation revealed a $91 \%$ internalization efficacy. This suggests that functional alterations demonstrated by this study were due to exosome-induced cell-population, and not individual cell level effects.

Then, we determined whether the internalized exosomes could induce a melanoma-like malignant transformation of the MSCs. By employing two complementary cell-counting methods, we found that proliferation rate of the MSCs significantly accelerated $72 \mathrm{~h}$ after exosome exposure (Figure 2C). By flow cytometry, we also showed that the exosome-treated MSCs exhibited a partial resistance to the cell death-inducing effects of $100 \mathrm{ng} / \mathrm{ml}$ tumor necrosis factor- $\alpha(\mathrm{TNF} \alpha)$ as the fraction of the dead cells was significantly decreased in these cultures (Figure 2D).

Since the exosomes were isolated from melanoma cells, we were then intrigued to uncover whether the above alterations (which all argue for the malignant transformation of the susceptible cells) also resulted in de novo appearance of melanoma-specific features in the transformed MSCs. To answer this question, expression of the melanoma-specific markers MLANA and MITF were investigated. By QRT-PCR, we found that mRNA transcript levels of both markers elevated markedly in MSCs upon exosome treatment (Figure 2E and Figure S2), albeit the kinetics of elevation of the two molecules were slightly different. Moreover, in good accordance with the mRNA data, immunofluorescence labeling showed that exosome exposure markedly increased the expression of MLANA at the protein level as well (Figure 2F).

Next, we assessed whether the above effects of exosomes inducing a malignant-like transformation of the MSCs was accompanied by a cellular-molecular oncogenic reprogramming of the target cells. Naïve MSCs were exposed to a standardized volume of exosomes for various time intervals (to avoid the experimental fluctuations, we collected pooled samples from multiple independent in vitro experiments). Then, samples were subjected to QRT-PCR analysis using a self-designed panel of 40 oncogenes and tumor suppressor genes which were previously suggested to play a role in melanoma progression (Table 3 ).

As shown in Figure 3A, gene expression pattern of MSCs exposed to melanoma exosomes, exhibited a clear oncogenic dominance (compared to the non-exposed cells). This was verified by statistical analysis of the mean relative gene expression levels of all molecules investigated. Statistically higher values were obtained in the case of exosome-treated cells $(p=1.9 \times$ $10^{-5}, p=0.031$ and $p=2.3 \times 10^{-8}$ for the 6,24 , and $72 \mathrm{~h}$ time points, respectively).

As mentioned above, Kleffel et al. has recently shown that melanoma cell subpopulations which overexpress PD-1, quite intriguingly exhibit remarkably increased invasiveness and aggressive growth properties (15). However, the authors did not define the factor(s) which induced the above PD-1 overexpression. Since the above finding strongly suggested an "MSC re-education" capacity of melanoma exosomes to induce malignant-like behavior, we next assessed the expression of PD-1 in MSC cultures.

As expected, only insignificant PD-1 expression (both at the mRNA and protein levels) could be identified in control, nontreated MSCs. In contrast, a marked, significant, and timedependent elevation of PD-1 expression was detected upon exosome treatment by QRT-PCR (Figure 3B), Western blot and an immunocytochemical analysis (Figures 3C,D). Further, by employing super-resolution microscopy, we were able to identify a dramatic upregulation of PD-1 at the single molecular level in exosome-treated MSCs (Figure 3E).

Importantly, since proteomics analysis did not identify the presence of PD-1 in exosomes, these data suggest that the high PD-1 protein content in exosome-exposed MSCs was a result of de novo induction and not of exosome-mediated molecular transfer. Our findings therefore suggest that melanoma exosomemediated "re-education" of the cells resulted in a novel MSC population which could be identified as $\mathrm{MSC}^{\mathrm{PD}-1+}$.

\section{B16F1 Exosomes Augment in vivo Tumorigenesis and Tumor Progression}

After presenting evidence for the in vitro tumorigenic induction potential of exosomes on cultured MSCs, we hypothesized that this phenomenon could be identified in vivo as well. To probe this assumption, we employed the well-known animal model, routinely used in our laboratories (46), in which tumors, developed mostly in the lungs, are induced in mice by intravenous administration of mouse B16F1 melanoma cells (to the tail vein). Then, tumor-bearing mice received buffer, or exosomes isolated from the same B16F1 melanoma cells, or exosome-induced $\mathrm{MSC}^{\mathrm{PD}-1+}$ cells.

Notably, the exosome-related groups (i.e., exosome, $\mathrm{MSC}^{\mathrm{PD}-1+}$ ) were characterized by a markedly increased size of tumor-covered lung tissues (the increase proved to be significant in the $\mathrm{MSC}^{\mathrm{PD}-1+}$ group) (Figure 4A). Of further 


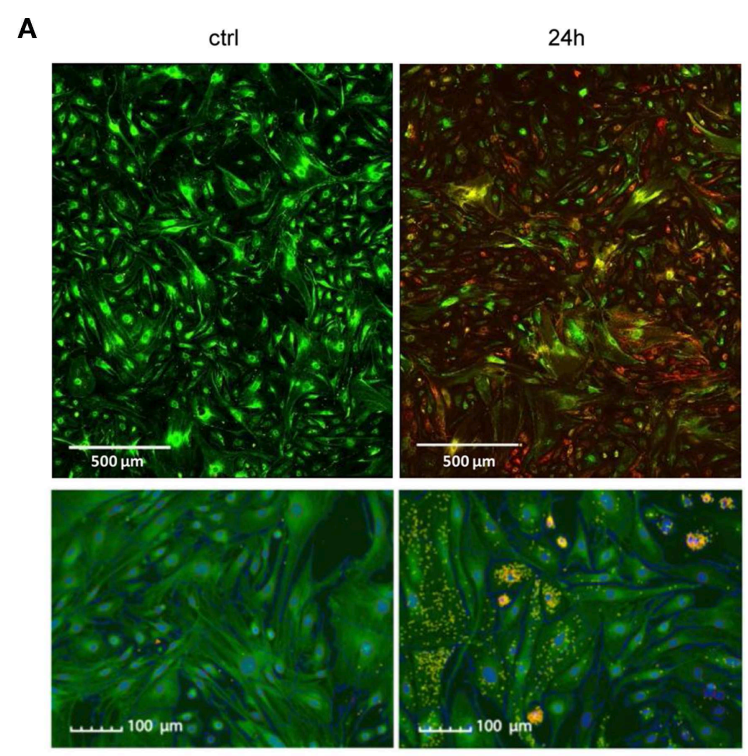

A

E

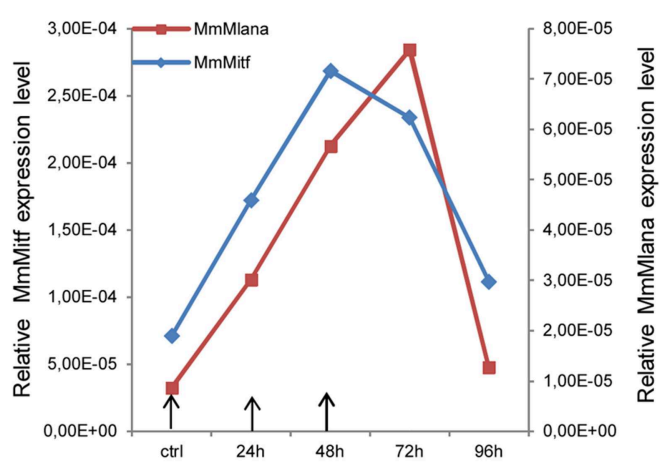

B

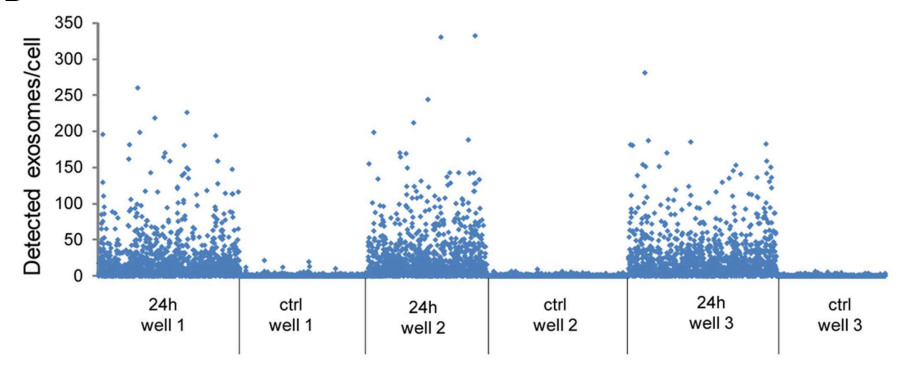

C
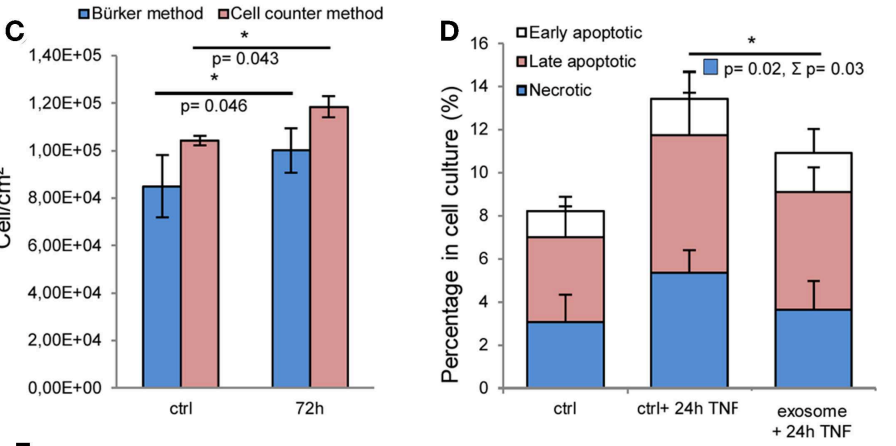

$\mathbf{F}$

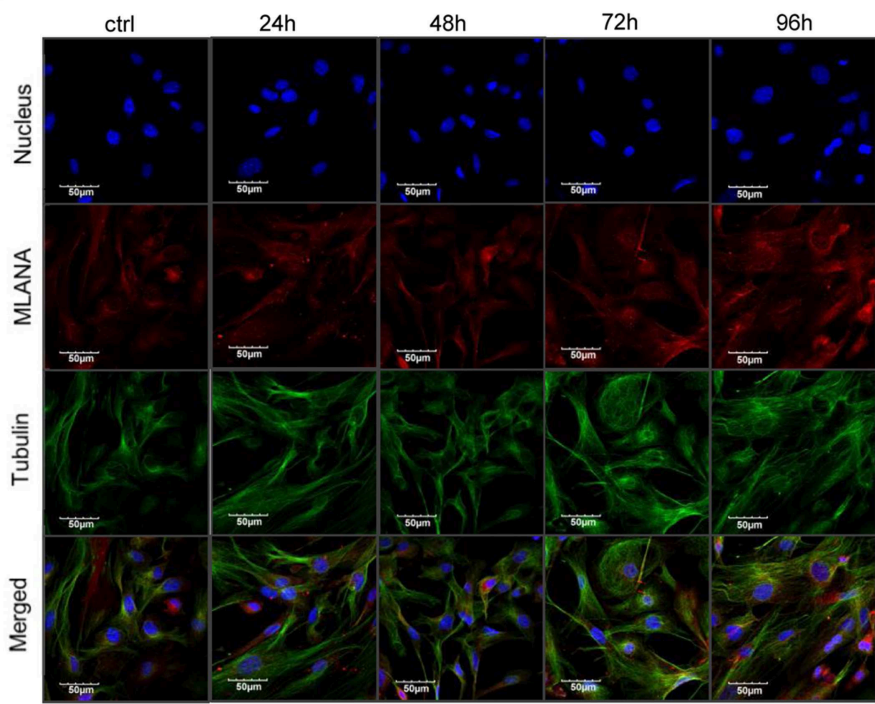

FIGURE 2 | Internalized melanoma exosomes induce malignant transformation of the recipient MSCs. (A) Fluorescent images of exosome uptake by MSCs. DiO (green lipid dye)-labeled cells were exposed to Dil (red lipid dye)-labeled exosomes for $24 \mathrm{~h}$ and fixed in $4 \%$ PFA. Control cells were treated in the same manner without exosomes. Pictures were acquired by an Operetta high content screening system (Perkin Elmer). Lower two images represent an experiment, where nuclei were counterstained with DAPI. Exosomes were detected with a customized version of A-trous wavelet transform, and were highlighted with yellow. (B) Quantitative analysis of exosome uptake. The graph shows the number of detected exosomes ( $\mathrm{Y}$ axis) in each cell ( $\mathrm{X}$ axis) in three exosome-exposed (24 h) and three control cell cultures (ctrl). (C) Cell proliferation assay of exosome-exposed MSCs. Cells were plated at $1 \times 10^{4} \mathrm{cell} / \mathrm{cm}^{2}$ density in control and exosome-exposed cultures, and $72 \mathrm{~h}$ after the exosome treatment, the cell number was determined by manual counting and by an automated cell counter. Both methods showed a significantly increased cell proliferation of exosome-treated cells. Results are presented as mean $\pm \mathrm{SD}(n=3)$. (D) Apoptosis analysis of exosome-exposed MSCs by flow cytometry. Exosome-pre-exposed cells were treated by $100 \mathrm{ng} / \mathrm{ml} \mathrm{TNF} \alpha$ for $24 \mathrm{~h}$, stained with Annexin V-FITC and propidium iodide. They were analyzed by flow cytometry both in the case of the TNF $\alpha$-treated and the untreated control cell cultures. The graph represents the percentage of early apoptotic, late apoptotic and necrotic cells. Both TNF $\alpha$-induced total cell death $(\Sigma)$ and necrosis were significantly lower $(\Sigma \mathrm{p}=0.03$ and $p=0.02$, respectively) in exosome-pretreated cell cultures compared to the corresponding control cells. Results are presented as mean $+\mathrm{SD}(n=3)$. (E) QRT-PCR analysis of Mlana and Mitf in MSC cultures treated by exosomes in every $24 \mathrm{~h}$ indicated by arrows in the graph. The expression of both mRNAs increased after exosome exposure, but they showed different kinetics. (F) Fluorescent immunocytochemistry of MLANA in exosome-exposed MSC cultures using a primary rabbit antibody to MLANA and a secondary AlexaFluor555-conjugated antibody to rabbit lgG (red). $\alpha$-tubulin network of cells was directly labeled by an AlexaFluor488-conjugated antibody (green) and the nuclei were stained with DAPI (blue). 
TABLE 3 | List of genes investigated by a self-designed oncopanel.

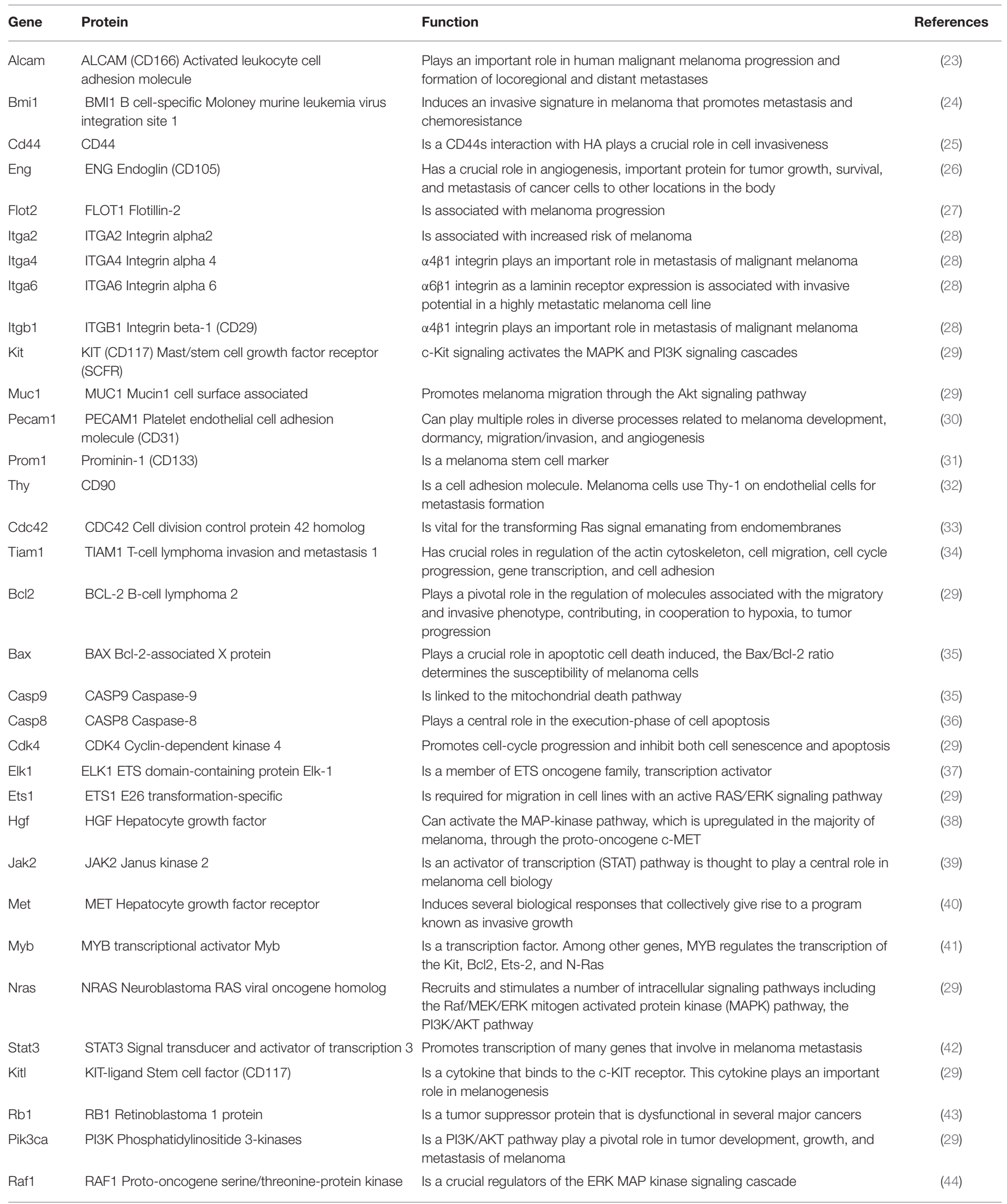


TABLE 3 | Continued

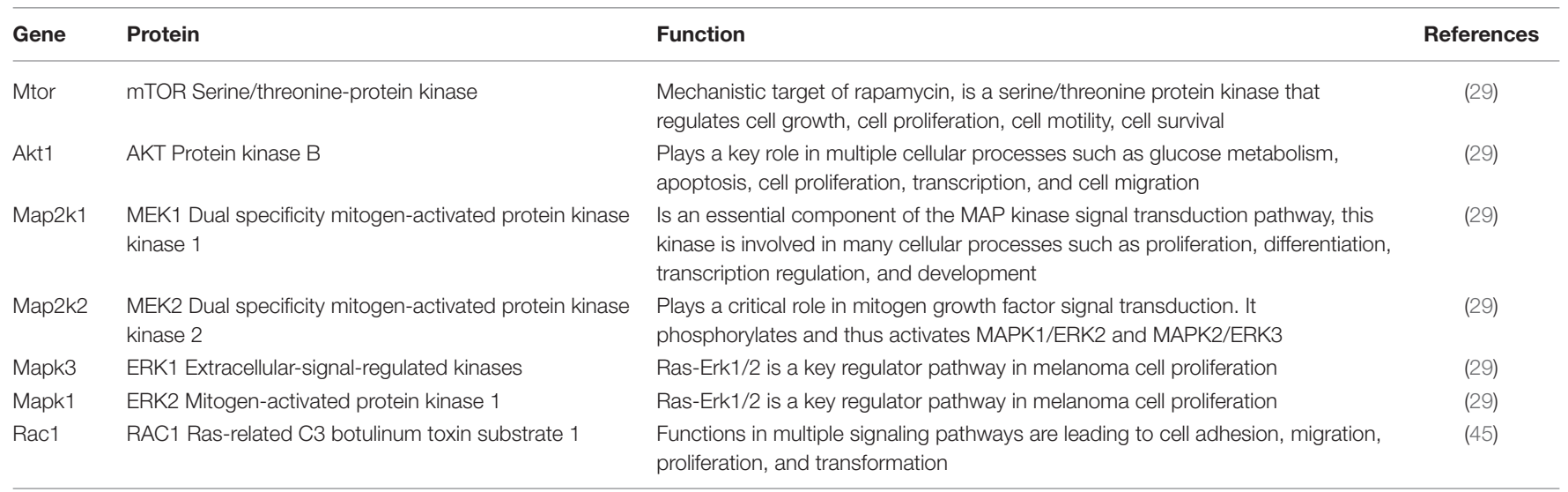

importance, we also found that in both exosome-related groups, the numbers of distant metastases were significantly elevated when compared to the control (Figure 4B), and diameter of metastases associated blood vessels significantly increased in exosome and $\mathrm{MSC}^{\mathrm{PD}-1+}$ injected groups of tumor bearing mice (Figure S3). Using of cytokine and chemokine arrays, tumor supportive cytokine and chemokine levels were elevated in exosome related groups (Figure S4). As we have seen during our previous studies (46), these metastases were mostly localized to the ovaries and kidneys (and very rarely to the lymph nodes) of control tumor-bearing animals. However, besides these sites, the presence of exosomes resulted in frequent metastases in the lymph nodes and, as a new location, in the liver. Interestingly, in MSC $^{\mathrm{PD}-1+}$ treated mice, exosome-transformed MSCs could be identified in the para-aortic lymph nodes by FISH (Figure 4C) verifying the successful in vivo adherence of $\mathrm{MSC}^{\mathrm{PD}-1+}$ cells.

Lung tissues of the different groups were then subjected to indepth expressional profiling 14 days after injection of exosomes or MSC $\mathrm{MD}^{\mathrm{PD}} 1+$ cells. Namely, QRT-PCR analysis was performed using a self-designed panel of 40 genes; besides housekeeping genes, we assessed expression of (i) proto-oncogenes; (ii) genes reportedly involved in malignant transformation; and/or (iii) genes found to be involved in melanoma development and progression.

Hierarchical cluster analysis (HCA) of the gene expression patterns clearly showed a robust proto-oncogenic dominance in lung samples of the exosome-related groups when compared to the control tissues. Indeed, we identified significant de novo induction of 26 and 23 genes: Alcam1, Eng, Flot2, Itga4, Itga6, Kit, Pecam1, Prom1, Thy, Cdc42, Tiam1, Bcl2, Bax, Casp9, Casp8, Ets1, Hgf, Jak2, Met, Myb, Map2k1, Map2k2, Mapk1 in both group and Elk1, Rb1, Itga2 in the exosome group. An additional six and three genes were identified with at least 10-fold increase (Cd44, Itgb1, Muc1, Pik3ca, Akt1, Rac1 in the exosome group and Cd44, Itgb1, Rac1 in the $\mathrm{MSC}^{\mathrm{PD}-1+}$ group). Furthermore, six and eight genes showed at least two-fold mRNA level elevations (Bmi1, Cdk4, Stat3, Kitl, Raf1, Mtor and Bmi1, Muc1, Cdk4, Stat3, Kitl, Pik3ca, Mtor, Akt1, respectively). Figure 4D presents a heatmap of indicative data of overexpressed genes induced by exosome exposure.
Furthermore, we showed that, besides the above genes, expression of PD-1 was also significantly increased in both exosome-related groups (Figure 4E). Notably, although mRNA transcript level of PD-1 was close to two-fold in $\mathrm{MSC}^{\mathrm{PD}-1+}$ lung tissues in comparison to samples in the exosome group, the difference was not significant (most probably due to the large inter-animal variability and standard error).

We constructed a Venn-diagram (Figure 4F) to show all possible logical connections between the various gene expression patterns presented in Figure 4D. Importantly, according to cluster analysis, we could not identify a single gene which was missing from the exosome-related groups in comparison to the control tumor-bearing mice. In other words, whereas these exosome-related groups did exhibit gene expression profiles which were characteristic only for them, such individual profiles could not be detected in the control group.

Specifically, the following gene expression patterns were defined:

- three genes (Elk1, Rb1, Igta2) were exclusively induced only in the exosome-treated group;

- twenty-three genes (Prom1, Tiam1, Bcl2, Bax, Casp9, Hgf, Jak2, Met, Mapk2k2, Alcam1, Eng, Flot2, Itga4, Itga6, Kit, Pecam1, Thy, Cdc42, Casp8, Ets1, Myb, Map2k1, Mapk1) were found to be upregulated both in the exosome and $\mathrm{MSC}^{\mathrm{PD}-1+}$ cell treated groups;

- fourteen genes (Bmi1, Cd44, Itgb1, Muc1, Cdk4, Nras, Sat3, Kitl, Raf1, Mtor, Akt1, Mapk3, Pik3ca, Rac1) were found to be upregulated in all three groups.

Finally, it should be noted that the dramatic gene expression alterations seen in the exosome-related groups were exclusively due to the presence of the exosomes as the "MSC $\mathrm{PD}-1+$ only" cluster contained no genes (Figure 4F).

\section{Melanoma-Derived Exosomes Promote Tumorigenic and Cell Survival Signaling Pathway(s)}

Figure 5 shows the map of interaction pathways based on overexpressed molecules (red symbols) in in vivo experiments. We detected overexpressed elements of three main pathways 


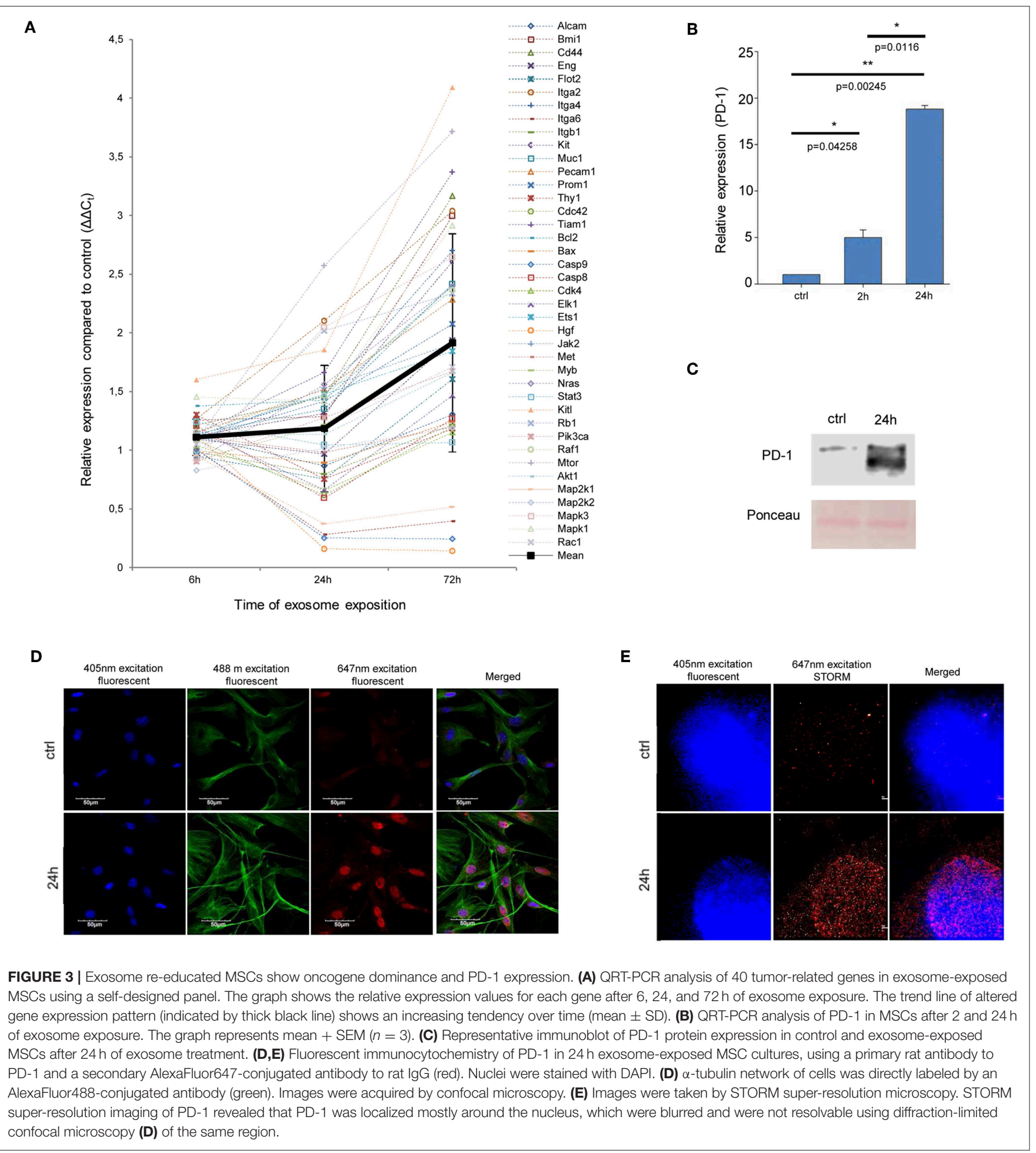

which do participate in tumor progression and metastasis formation (47). Using the IPA Path Designer Grow tool, we generated an interaction map which contains proteins (or their established complexes) encoded by the overexpressed genes, and exosomal miRNAs and proteins (gray boxes) which were previously shown to control or affect the marked signaling molecules.

To demonstrate these results and to explore the potential causative exosomal factors, we again performed an IPA. The analysis allowed further refinement of the underlying molecular 


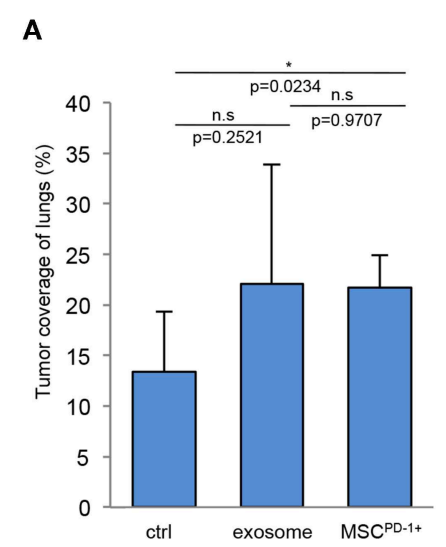

D

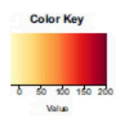

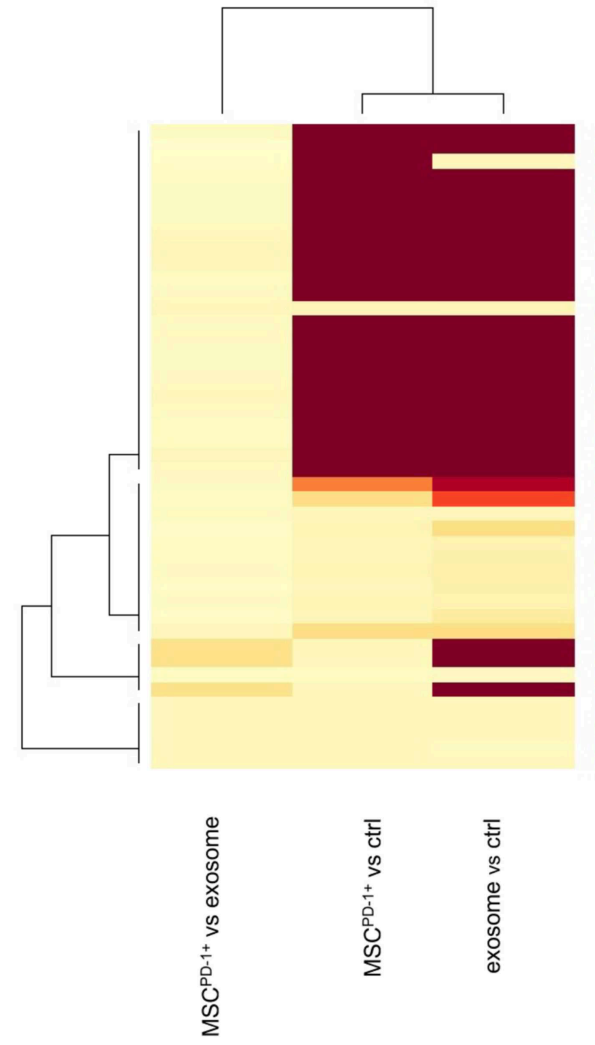

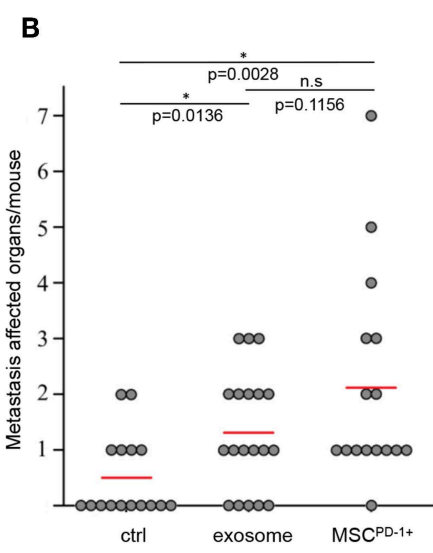

C

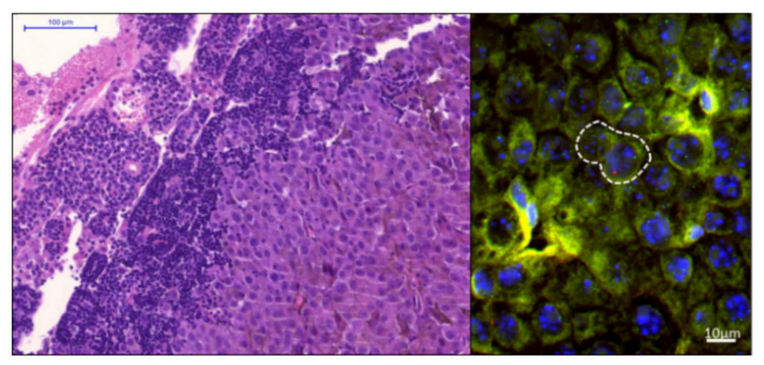

E

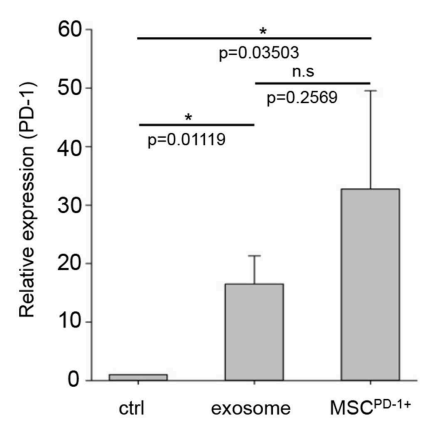

$\mathbf{F}$

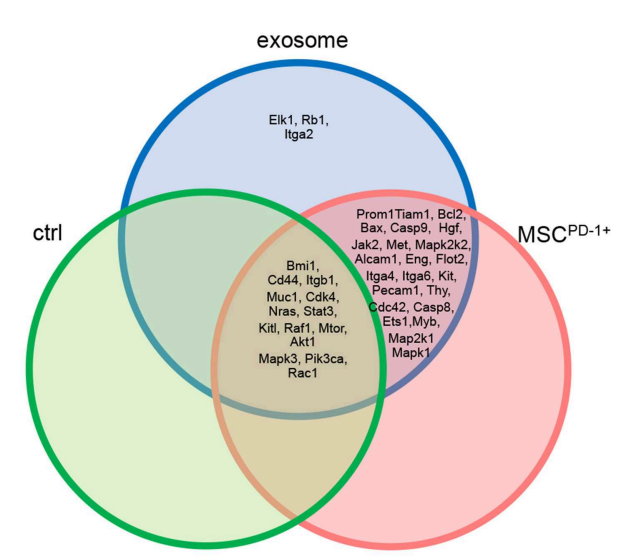

FIGURE 4 | Melanoma exosomes promote tumor progression and metastasis formation in vivo. (A) Tumor coverage of lungs on day 15 in different animal groups. The graph represents mean $+\mathrm{SD}(n=3)$. (B) Number of distant metastases on day 25. One dot represents one animal in each group, red lines show the average number of metastases per animal. (C) FISH analysis of a paraortic lymph node metastases, which showed the presence of exosome-exposed MSC. Y chromosome (red dot) of the male mouse-derived MSC was detected in the metastases of a female mouse. (D) Heatmap and cluster analysis of gene expression pattern, which show protooncogenic dominance in exosome or $\mathrm{MSC}^{\mathrm{PD}-1+}$ groups. Robust hierarchical clustering based on fold changes in the gene expression data between selected groups divide the treated groups into several upper classes. Groups treated by exosomes or MSC ${ }^{\mathrm{PD}-1+}$ were compared to untreated controls. Exosomes MSCPD-1+ treatments were closely related. (E) QRT-PCR analysis of PD-1 in the lung samples using TagMan probes $(n=3)$. (F) The Venn diagram shows possible relations between a finite collections of different sets of genes measured during gene expression profiling (D)

processes and pathways involved in the summarized effect of this gene expression profile. The previously described 40 -gene QRT-PCR panel was enriched in genes related to cellular movement and migration, cell survival and connective tissue development and function, involving tumor cells. Additional processes included growth and proliferation of tumor cells as well as the PD-1:PD-L1 interaction (and its consequences). Focusing on PD-1 and mTOR pathway, a network was 


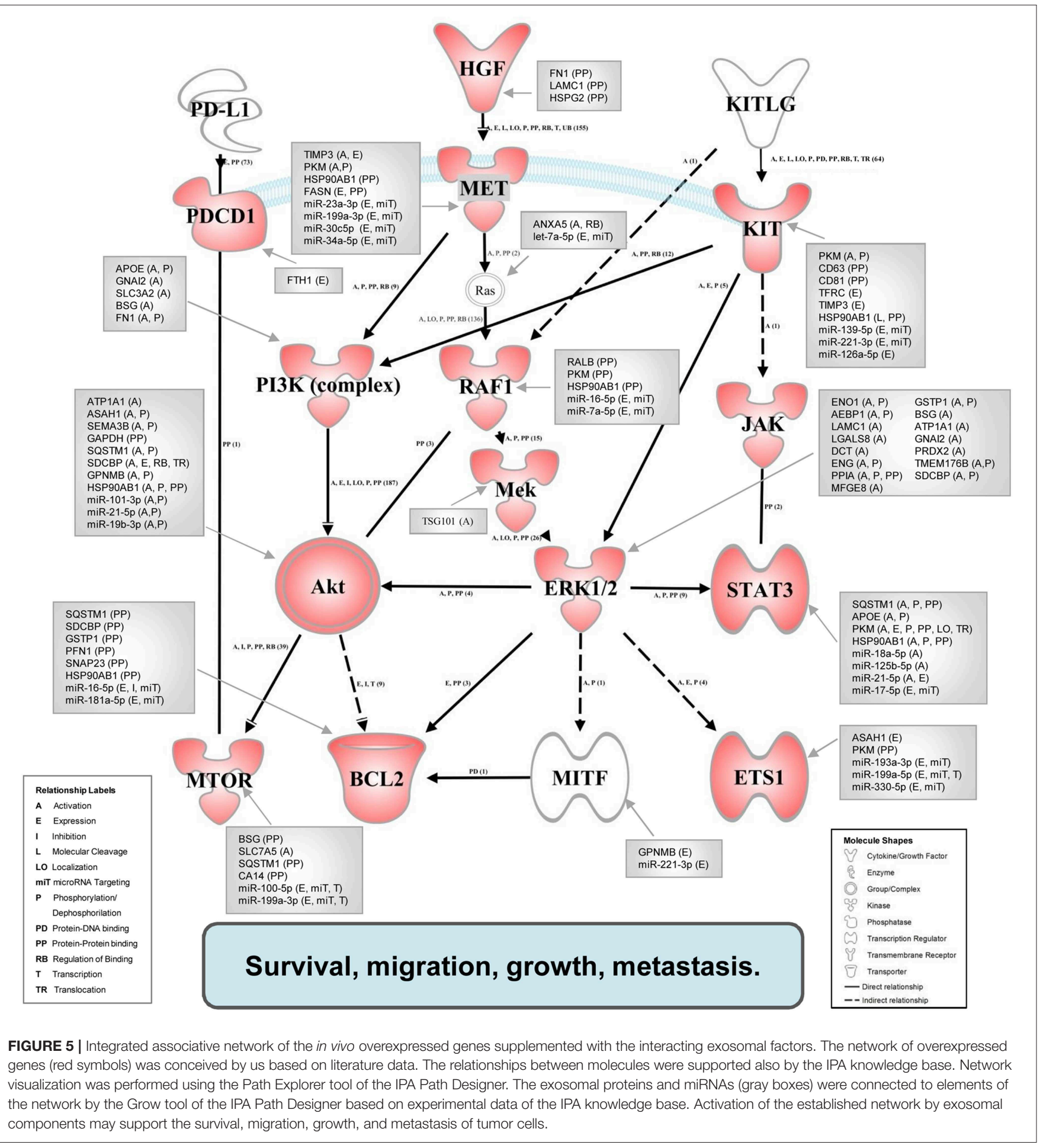

built from the overexpressed genes, and their complexes known to be related to tumor progression using the Path Explorer tool in the IPA Path Designer. Then a list was generated that contained the exosomal proteins detected by MS and the exosomal miRNAs identified by SOLiD sequencing. The Grow tool in the IPA Path Designer revealed significant interactions between the exosome-induced expression network and the molecular content of these vesicles. The resulting interaction network shows both direct and indirect interactions, but it contains only experimentally observed relationships based on the Ingenuity Knowledge Base. This network demonstrates that 61 exosomal molecules (Table 4) 
TABLE 4 | List of exosomal proteins and miRNAs connected to the integrated associative network by IPA Path Designer.

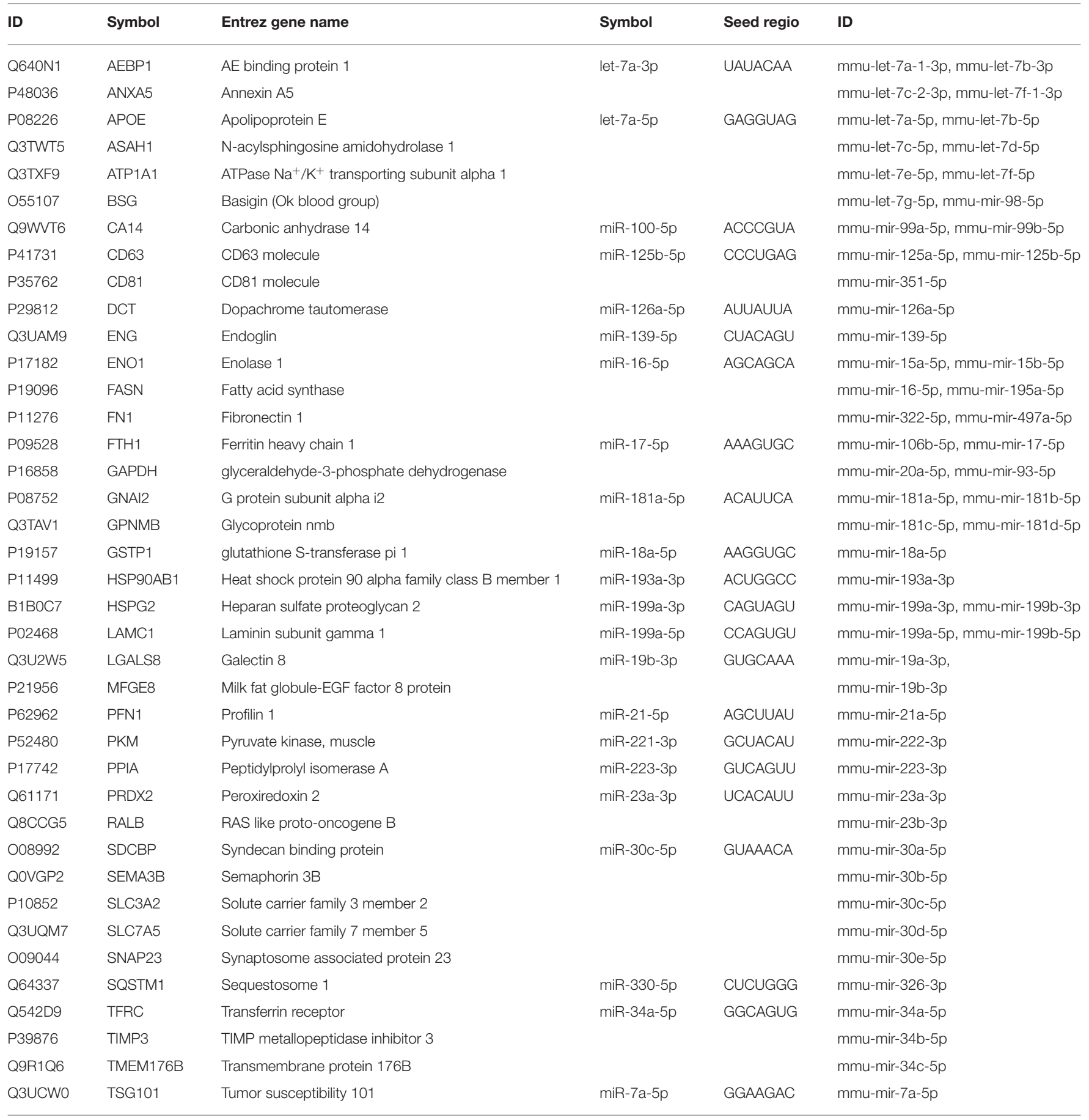

may affect tumor progression through pathways controlled by key components including MET, Ras, RAF1, Mek, ERK1/2, MITF, BCL2, PI3K, Akt, mTOR, PD-1, KIT, JAK STAT3, or ETS1.

Taken together, these findings demonstrate that interaction between exosomes and MSCs induces a tumor-like phenotype with PD-1 overexpression of naïve MSC in vitro and a fast tumor progression in vivo.

\section{DISCUSSION}

Considering the fact that metastatic complications are responsible for almost $90 \%$ of cancer-driven mortality (48), a deep understanding of tumor metastatic processes is one of the most important challenges in both fundamental and applied research directions in tumor biology. Whilst certain organotropic aspects of tumor cells were addressed by Paget's 
"seed-and-soil" hypothesis in the early nineteenth century (49), the detailed description of the spatiotemporal characteristics of the metastatic processes requires an extended model. MSCs, as multipotent stromal cells, are important players in dynamic tumor microenvironment (12). MSCs could stimulate angiogenesis via paracrine signaling and participate in metastasis formation to promote the epithelial-mesenchymal transition (12). Consequently, in the last decade, numerous studies suggested the importance of soluble and vesicle-like components of the tumor microenvironment in epithelial-mesenchymal transition as crucial factors in the metastatic establishment (50). Among these, recent findings of intercellular communication mechanisms unquestionably highlighted the crucial role of EVs (e.g., exosomes) serving as "information packages" within the coordinated cell-to-cell signaling (51).

In the current study, we demonstrate that MSCs, widely abundant in solid tumors as well as in healthy tissues, undergo a marked re-education process upon communication with metastatic cancer cells via exosome-mediated information transfer. This transformation process results in characteristic response-patterns corresponding to a given cancerous cell lines (12).

First, we intended to precisely define the EV population which we later employed via in vitro and in vivo experimental approaches. Besides applying the experimental requirements of the International Society for Extracellular Vesicles (ISEV) (i.e., determination of shape, size distribution and protein markers) (52), we thoroughly defined the miRNA and protein content of melanoma exosomes. These systematic assays concluded that the isolated vesicles contained factors, which may regulate signaling pathways related to cell death and survival, cellular movement, development and cell proliferation. Given the appropriate uptake of these vesicles by the targeted, multipotent recipient cells, it is highly probable that most signaling pathways mentioned above were significantly affected. In accordance with previous results in the literature, we showed successful internalization of exosomes by recipient MSCs. As expected, following $72 \mathrm{~h}$ of exposure, MSCs displayed accelerated proliferation presumably initiated by the transforming exosome cargo. Moreover, melanoma exosomes were able to hinder the induction of the cell death response commonly triggered by TNF $\alpha$. TNF $\alpha$ is a frequently identified, soluble, tumor-suppressing factor in cancerous microenvironments (53). This inhibition strongly suggested that exosomes initialize and sustain malignant transformation of MSCs.

Further, we described numerous, exosome-induced phenotypic manifestation of the above malignant re-education process in the recipient cells. Apart from identifying tumor markers, the transformation of exposed cells is characterized by markedly modified gene expression patterns. As discussed previously, specific gene expression alterations are often associated with specific steps of cancerous transformation. Malignant cell lines possess altered expression of genes responsible for apoptotic regulation ( $\mathrm{Bax}, \mathrm{Bcl} 2$, caspases), regulating metastasis (integrins, ITGA2-6, ITGB1, KIT) or various growth factors (ETS1, HGF, MET, etc.). Importantly, we successfully identified two melanoma-specific markers, i.e., MITF (Microphthalmia-associated transcription factor) and MLANA (Melanoma antigen recognized by T-cells) in the exosome-exposed MSCs. MLANA and its regulator MITF play a fundamental role in melanocyte development, tumor progression and are overexpressed in melanoma cells (54). The presence of these markers in exosome-exposed MSCs shows effective transfer of the encoded tumorigenic information, and may have a pivotal role in disease progression. Besides, we also identified a characteristic set of genes, belonging mostly to protooncogenes, whose expression was significantly elevated upon exosome treatment. Among these molecules, we emphasize the increased expression of CD44 which was previously assigned as cancer stem cell marker and of mTOR, a characteristic indicator of the engagement of the PD-1:PD-L1 pathway (15). Indeed, activation of mTOR signaling is particularly important. As it was previously demonstrated by Kleffel et al., overexpression of PD-1 in exceptionally aggressive melanoma subpopulations facilitated tumor progression by activating the mTOR signaling pathway (actually, this response was highly unexpected compared to that of the classic PD-1:PD-L1 dependent T-cell anergy, a crucial aspect of immunotherapy in the clinics) (15). Quite strikingly, in exosome treated MSCs, we detected a significant induction of PD-1 and mTOR. These novel data strongly support that these vesicles might trigger the formation of an aggressive, melanoma-like subpopulation of re-educated recipient MSCs.

Our findings therefore propose that the re-educated melanoma-like $\mathrm{MSC}^{\mathrm{PD}-1+}$ subpopulation, originating from multipotent tissue-derived MSCs, is an autonomous entity. It is characterized by typical properties of cancerous transformation such as hyperproliferation, resistance to apoptosis, expression of melanoma markers, proto-oncogenic gene expression patterns, and PD-1 over-expression. Indeed, those melanoma exosomes that induced in vitro generation of the melanoma-like $\mathrm{MSC}^{\mathrm{PD}-1+}$ cells, also facilitated in vivo metastasis formation in the lungs or other distant organs of treated animals. Actually, our findings that melanoma exosomes or exosome re-educated $\mathrm{MSC}^{\mathrm{PD}-1+}$ promote tumor progression, are in good accordance with previous finding of Kleffel et al. describing that shRNA or antibody mediated inhibition of PD-1 signaling inhibits metastasis formation and tumor progression in experimental animals (15). Assessment of the expression patterns of genes related to tumor progression in the exposed animal tissues revealed numerous cases of exosome-induced overexpression; this supports our hypothesis that melanoma exosomes should be considered as oncosomes (6).

Our results also demonstrated that melanoma exosomes also generated a characteristic signaling pattern in $\mathrm{MSC}^{\mathrm{PD}-1+}$ recipient population. As it was shown in numerous studies PI3K/Akt, Ras/MAPK, and STAT3 pathways are commonly activated in tumor cells $(47,55)$. In this study, based on our in vitro and in vivo results and literature data, we introduce a novel, comprehensive network of common tumorrelated proteins, such as PD-1, MET, RAF1, STAT3, BCL2, or mTOR. The network highlights not only the relationship of these elements, but also contains upstream exosomal regulating factors which may contribute to the activation of tumorigenic signaling, and hence, fast tumor progression. 
According to our best knowledge, this associative network between overexpressed genes and the potential exosomal inducers, is the first tumor progression signaling pattern which connected experimental response-patterns with experimentally detected exosomal-molecular-patterns. Further, our data also indicate that the above complexity of exosomal communication requires system-level approaches.

Finally, it is important to note that our conclusions are not based on in silico predictions exclusively, but rather on carefully designed and systematically executed in vitro and in vivo experiments, all suggesting that the specific, robust, molecular content of the isolated exosomes can indeed generate a unique intercellular niche responsible for the re-education of neighboring cells via oncogenic transformation. These reeducated melanoma-like $\mathrm{MSC}^{\mathrm{PD}-1+}$ cells, in turn, facilitate metastatic disease progression via ignition of a complex series of subsequent events both locally and systematically. Thus, based on our results and recently published, additional evidences about exosomes, there is an urgent demand to supplement and extend the outdated "seed-and-soil" hypothesis with the oncosomedriven re-education process.

\section{DATA AVAILABILITY STATEMENT}

All datasets generated for this study are included in the manuscript/Supplementary Files.

\section{ETHICS STATEMENT}

All animal experiments were performed in accordance with national (1998. XXVIII; 40/2013) and European (2010/63/EU) animal ethics guidelines. The experimental protocols were approved by the Animal Experimentation and Ethics Committee of the Biological Research Centre of the Hungarian Academy of Sciences and the Hungarian National Animal Experimentation and Ethics Board (clearance number: XVI./03521/2011 and XVI./78/2018).

\section{AUTHOR CONTRIBUTIONS}

EG-S: acquisition, analysis and interpretation of data (in vitro and in vivo experiments), drafting or revising the article. $\mathrm{MH}$ and GD: acquisition, analysis and interpretation of data, drafting or revising the article. IBN: histology. JM: acquisition, analysis

\section{REFERENCES}

1. Li HLL, Fan X, Houghton J. Tumor microenvironment: the role of the tumor stroma in cancer. J Cell Biochem. (2007) 101:805-15. doi: 10.1002/jcb.21159

2. Yang C, Robbins PD. The roles of tumor-derived exosomes in cancer pathogenesis. Clin Dev Immunol. (2011) 2011:842849. doi: 10.1155/2011/842849

3. Braicu C, Tomuleasa C, Monroig P, Cucuianu A, Berindan-Neagoe I, Calin GA. Exosomes as divine messengers: are they the Hermes of modern molecular oncology? Cell Death Differ. (2015) 22:34-45. doi: $10.1038 /$ cdd. 2014.130 and interpretation of data-in vivo QRT-PCR. ÁZ: acquisition, analysis and interpretation of data (MLANA, MITF). ÉH-G: acquisition, analysis and interpretation of Proteomics data. RK: providing and supervising of stem cell protocols. IN: acquisition, analysis and interpretation of miRNA data. $\mathrm{PH}$ : supervising of machine learning. ÁB: high content screening. ÁS: acquisition, analysis and interpretation of machine learning data. MK: acquisition, analysis and interpretation of confocal data. TP: drafting or revising the article, PD-1 QRT-PCR. BB: acquisition, analysis of data (in vitro QRT-PCR). ME: STORM microscopy. ZS: AFM microscopy. ZV: acquisition, analysis and interpretation of apoptosis data. EB: drafting or revising the article. LK: clinical relevance and drafting or revising the article. TB: conception and design and drafting or revising the article. KB: conception and design, acquisition of data, analysis and interpretation of data, drafting or revising the article and founding acquisition.

\section{FUNDING}

This work was in part supported by the National Research, Development and Innovation Fund of Hungary, financed under the NKFI-6-K funding scheme (11493 project), GINOP-2.3.2-15-2016-00015, GINOP-2.2.1-152017-00052, János Bolyai Research Scholarship of the Hungarian Academy of Sciences ÚNKP-19-4 New National Excellence Program of the Ministry for Innovation and Technology and University of Szeged Open Access Fund. The dSTORM experiments were funded by the Hungarian Brain Research Program (KTIA_13_NAP-A-I/14) and the GINOP-2.3.2-15-2016-00036.

\section{ACKNOWLEDGMENTS}

The authors wish to thank Gábor Braunitzer and Csaba Vizler, for scientific discussion, Annamária Marton for technical issues and adaptation of MSC isolation protocol, Lilla Pintér for technical support.

\section{SUPPLEMENTARY MATERIAL}

The Supplementary Material for this article can be found online at: https://www.frontiersin.org/articles/10.3389/fimmu. 2019.02459/full\#supplementary-material
4. Yáñez-Mó M, Siljander PR, Andreu Z, Zavec AB, Borràs FE, Buzas E, et al. Biological properties of extracellular vesicles and their physiological functions. J Extracell Vesicles. (2015) 4:27066. doi: 10.3402/jev.v4. 27066

5. Harmati M, Tarnai Z, Decsi G, Kormondi S, Szegletes Z, Janovak L, et al. Stressors alter intercellular communication and exosome profile of nasopharyngeal carcinoma cells. J Oral Pathol Med. (2017) 46:259-66. doi: $10.1111 /$ jop. 12486

6. Meehan B, Rak J, Di Vizio D. Oncosomes - large and small: what are they, where they came from? J Extracell Vesicles. (2016) 5:33109. doi: 10.3402/jev.v5.33109 
7. Minciacchi VR, Freeman MR, Di Vizio D. Extracellular vesicles in cancer: exosomes, microvesicles and the emerging role of large oncosomes. Semin Cell Dev Biol. (2015) 40:41-51. doi: 10.1016/j.semcdb.2015. 02.010

8. Chen PM, Yen ML, Liu KJ, Sytwu HK, Yen BL. Immunomodulatory properties of human adult and fetal multipotent mesenchymal stem cells. $J$ Biomed Sci. (2011) 18:49. doi: 10.1186/1423-0127-18-49

9. Ridge SM, Sullivan FJ, Glynn SA. Mesenchymal stem cells: key players in cancer progression. Mol Cancer. (2017) 16:31. doi: 10.1186/s12943-017-0597-8

10. Suzuki K, Sun R, Origuchi M, Kanehira M, Takahata T, Itoh J, et al. Mesenchymal stromal cells promote tumor growth through the enhancement of neovascularization. Mol Med. (2011) 17:579-87. doi: 10.2119/molmed.2010.00157

11. Mishra PJ, Mishra PJ, Glod JW, Banerjee D. Mesenchymal stem cells: flip side of the coin. Cancer Res. (2009) 69:1255-8. doi: 10.1158/0008-5472.CAN-08-3562

12. Baglio SR, Lagerweij T, Pérez-Lanzón $M$, Ho XD, Léveillé N, Melo $\mathrm{SA}$, et al. Blocking tumor-educated MSC paracrine activity halts osteosarcoma progression. Clin Cancer Res. (2017) 23:3721-33. doi: 10.1158/1078-0432.CCR-16-2726

13. Peinado H, Alečković M, Lavotshkin S, Matei I, Costa-Silva B, MorenoBueno G, et al. Melanoma exosomes educate bone marrow progenitor cells toward a pro-metastatic phenotype through MET. Nat Med. (2012) 18:883-91. doi: $10.1038 / \mathrm{nm} .2753$

14. Kee D, McArthur G. Immunotherapy of melanoma. Eur J Surg Oncol. (2017) 43:594-603. doi: 10.1016/j.ejso.2016.07.014

15. Kleffel S, Posch C, Barthel SR, Mueller H, Schlapbach C, Guenova E, et al. Melanoma cell-intrinsic PD-1 receptor functions promote tumor growth. Cell. (2015) 162:1242-56. doi: 10.1016/j.cell.2015.08.052

16. Bersanelli M, Buti S. From targeting the tumor to targeting the immune system: transversal challenges in oncology with the inhibition of the PD-1/PDL1 axis. World J Clin Oncol. (2017) 8:37-53. doi: 10.5306/wjco.v8.i1.37

17. Szebeni GJ, Kriston-Pál É, Blazsó P, Katona RL, Novák J, Szabó E, et al. Identification of galectin-1 as a critical factor in function of mouse mesenchymal stromal cell-mediated tumor promotion. PLoS ONE. (2012) 7:411372. doi: 10.1371/journal.pone.0041372

18. Carpenter AE, Jones TR, Lamprecht MR, Clarke C, Kang I, Friman O, et al. CellProfiler: image analysis software for identifying and quantifying cell phenotypes. Genome Biol. (2006) 7:R100. doi: 10.1186/gb-2006-7-10-r100

19. Olivo-Marin JC. Extraction of spots in biological images using multiscale products. Pattern Recognit. (2002) 35:1989-96. doi: 10.1016/S0031-3203(01)00127-3

20. van de Linde S, Löschberger A, Klein T, Heidbreder M, Wolter S, Heilemann M, et al. Direct stochastic optical reconstruction microscopy with standard fluorescent probes. Nat Protoc. (2011) 6:991-1009. doi: 10.1038 /nprot.2011.336

21. Rees EJ, Erdelyi M, Kaminski-Schierle GS, Knight AE, Kaminski CF. Elements of image processing in localisation microscopy. J Opt. (2013) 15:094012. doi: 10.1088/2040-8978/15/9/094012

22. R Development Core Team. R: A Language and Environment for Statistical Computing. R Foundation for Statistical Computing, Vienna, Austria (2008).

23. Donizy P, Zietek M, Halon A, Leskiewicz M, Kozyra C, Matkowski R. Prognostic significance of ALCAM (CD166/MEMD) expression in cutaneous melanoma patients. Diagn Pathol. (2015) 10:86. doi: $10.1186 /$ s13000-015-0331-z

24. Ferretti R, Bhutkar A, McNamara MC, Lees JA. BMI1 induces an invasive signature in melanoma that promotes metastasis and chemoresistance. Genes Dev. (2016) 30:18-33. doi: 10.1101/gad.267757.115

25. Mummert ME, Mummert DI, Ellinger L, Takashima A. Functional roles of hyaluronan in B16-F10 melanoma growth and experimental metastasis in mice. Mol Cancer Ther. (2003) 2:295-300.

26. Muñoz R, Arias Y, Ferreras JM, Jiménez P, Langa C, Rojo MA, et al. In vitro and in vivo effects of an anti-mouse endoglin (CD105)-immunotoxin on the early stages of mouse B16MEL4A5 melanoma tumours. Cancer Immunol Immunother. (2013) 62:541-51. doi: 10.1007/s00262-012-1357-7

27. Hazarika P, McCarty MF, Prieto VG, George S, Babu D, Koul D, et al. Up-regulation of Flotillin-2 is associated with melanoma progression and modulates expression of the thrombin receptor protease activated receptor 1. Cancer Res. (2004) 64:7361-9. doi: 10.1158/0008-5472.CAN04-0823

28. Lee N, Barthel SR, Schatton T. Melanoma stem cells and metastasis: mimicking hematopoietic cell trafficking? Lab Investig. (2014) 94:13-30. doi: 10.1038/labinvest.2013.116

29. Ahmed KBR, Davies AM. New molecular targets for the systemic therapy of melanoma. In: Murph M, editor. Research on Melanoma - A Glimpse into Current Directions and Future Trends. London, UK: Intech (2011). p. 161-80.

30. Dunleavey JM, Xiao L, Thompson J, Kim MM, Shields JM, Shelton SE, et al. Vascular channels formed by subpopulations of PECAM1+ melanoma cells. Nat Commun. (2014) 5:5200. doi: 10.1038/ncomms6200

31. Madjd Z, Erfani E, Gheytanchi E, Moradi-Lakeh M, Shariftabrizi A, AsadiLari M. Expression of CD133 cancer stem cell marker in melanoma: a systematic review and meta-analysis. Int J Biol Markers. (2016) 31:118-25. doi: $10.5301 /$ jbm. 5000209

32. Schubert K, Gutknecht D, Köberle M, Anderegg U, Saalbach A. Melanoma cells use Thy-1 (CD90) on endothelial cells for metastasis formation. Am J Pathol. (2013) 182:266-76. doi: 10.1016/j.ajpath.2012.10.003

33. Stengel K, Zheng Y. Cdc42 in oncogenic transformation, invasion, and tumorigenesis. Cell Signal. (2011) 23:1415-23. doi: 10.1016/j.cellsig.2011.04.001

34. Minard ME, Kim LS, Price JE, Gallick GE. The role of the guanine nucleotide exchange factor Tiam1 in cellular migration, invasion, adhesion and tumor progression. Breast Cancer Res Treat. (2004) 84:21-32. doi: 10.1023/B:BREA.0000018421.31632.e6

35. Raisova M, Hossini AM, Eberle J, Riebeling C, Wieder T, Sturm I, et al. The Bax/Bcl-2 ratio determines the susceptibility of human melanoma cells to CD95/Fas-mediated apoptosis. J Invest Dermatol. (2001) 117:333-40. doi: 10.1046/j.0022-202x.2001.01409.x

36. Stupack DG. Caspase- 8 as a therapeutic target in cancer. Cancer Lett. (2013) 332:133-40. doi: 10.1016/j.canlet.2010.07.022

37. Chen J, Zhang XD. Nanodelivery of anticancer agents in melanoma: encouraging, but a long way to go. In: Hamblin M, Avci P, Prow T, editors. Nanoscience in Dermatology. Cambridge: Academic Press (2016). p. 189-202. doi: 10.1016/B978-0-12-802926-8.00015-X

38. Hügel R, Muendlein A, Volbeding L, Drexel H, Richtig E, Wehkamp U, et al. Serum levels of hepatocyte growth factor as a potential tumor marker in patients with malignant melanoma. Melanoma Res. (2016) 26:354-60. doi: 10.1097/CMR.0000000000000269

39. Nicholas C, Lesinski GB. The Jak-STAT signal transduction pathway in melanoma. In: Tanaka Y, editor. Breakthroughs in Melanoma Research. London, UK: IntechOpen. (2011). p. 283-306. doi: 10.5772/18876

40. Al-U'datt DGF, Al-Husein BAA, Qasaimeh GR. A mini-review of c-Met as a potential therapeutic target in melanoma. Biomed Pharmacother. (2017) 88:194-202. doi: 10.1016/j.biopha.2017.01.045

41. Ramsay RG, Gonda TJ. MYB function in normal and cancer cells. Nat Rev Cancer. (2008) 8:523-34. doi: 10.1038/nrc2439

42. Cao HH, Chu JH, Kwan HY, Su T, Yu H, Cheng CY, et al. Inhibition of the STAT3 signaling pathway contributes to apigenin-mediated anti-metastatic effect in melanoma. Sci Rep. (2016) 6:21731. doi: 10.1038/srep21731

43. Roesch A, Becker B, Meyer S, Hafner C, Wild PJ, Landthaler M, et al. Overexpression and hyperphosphorylation of retinoblastoma protein in the progression of malignant melanoma. Mod Pathol. (2005) 18:565-72. doi: 10.1038/modpathol.3800324

44. Kyriakis JM, App H, Zhang XF, Banerjee P, Brautigan DL, Rapp UR, et al. Raf-1 activates MAP kinase-kinase. Nature. (1992) 358:417-21. doi: $10.1038 / 358417 \mathrm{a} 0$

45. Li A, Machesky L. Rac1 cycling fast in melanoma with P29S.M. Pigment Cell Melanoma Res. (2013) 26:289-90. doi: 10.1111/pcmr.12074

46. Buzás K, Marton A, Vizler C, Gyukity-Sebestyén E, Harmati M, Nagy K, et al. Bacterial sepsis increases survival in metastatic melanoma: Chlamydophila pneumoniae induces macrophage polarization and tumor regression. J Invest Dermatol. (2016) 136:862-5. doi: 10.1016/j.jid.2015.12.032

47. Flaherty KT, Hodi FS, Fisher DE. From genes to drugs: targeted strategies for melanoma. Nat Rev Cancer. (2012) 12:349-61. doi: 10.1038/nrc3218

48. Chaffer CL, Weinberg RA. A perspective on cancer cell metastasis. Science. (2011) 331:1559-64. doi: 10.1126/science.1203543 
49. Paget $\mathrm{S}$. The distribution of secondary growths in cancer of the breast. Lancet. (1889) 1:571-3. doi: 10.1016/S0140-6736(00)49915-0

50. Gopal SK, Greening DW, Rai A, Chen M, Xu R, Shafiq A, et al. Extracellular vesicles: their role in cancer biology and epithelial-mesenchymal transition. Biochem J. (2017) 474:21-45. doi: 10.1042/BCJ20160006

51. Tkach M, Théry C. Communication by extracellular vesicles: where we are and where we need to go. Cell. (2016) 164:1226-32. doi: 10.1016/j.cell.2016.01.043

52. Lötvall J, Hill AF, Hochberg F, Buzás E, Di Vizio D, Gardiner C, et al. Minimal experimental requirements for definition of extracellular vesicles and their functions: a position statement from the International Society for Extracellular Vesicles. J Extracell Vesicles. (2014) 3:26913. doi: 10.3402/jev.v3.26913

53. Balkwill F. Tumour necrosis factor and cancer. Nat Rev Cancer. (2009) 9:361-71. doi: 10.1038/nrc2628

54. Schepsky A, Bruser K, Gunnarsso GJ, Goodall J, Hallsson JH, Goding CR, et al. The microphthalmia-associated transcription factor Mitf interacts with betacatenin to determine target gene expression. Mol Cell Biol. (2006) 26:8914-27. doi: 10.1128/MCB.02299-05
55. Chen J, Shao R, Zhang XD, Chen C. Applications of nanotechnology for melanoma treatment, diagnosis, and theranostics. Int J Nanomedicine. (2013) 8:2677-88. doi: 10.2147/IJN.S45429

Conflict of Interest: The authors declare that the research was conducted in the absence of any commercial or financial relationships that could be construed as a potential conflict of interest.

Copyright (c) 2019 Gyukity-Sebestyén, Harmati, Dobra, Németh, Mihály, Zvara, Hunyadi-Gulyás, Katona, Nagy, Horváth, Bálind, Szkalisity, Kovács, Pankotai, Borsos, Erdélyi, Szegletes, Veréb, Buzás, Kemény, Bíró and Buzás. This is an openaccess article distributed under the terms of the Creative Commons Attribution License (CC BY). The use, distribution or reproduction in other forums is permitted, provided the original author(s) and the copyright owner(s) are credited and that the original publication in this journal is cited, in accordance with accepted academic practice. No use, distribution or reproduction is permitted which does not comply with these terms. 
K Buzás et al.

C. Pneumoniae Increases Survival in Melanoma

Nicholas G. Martin ${ }^{1}$ and Sarah E. Medland ${ }^{7}$

${ }^{1}$ QIMR Berghofer Medical Research Institute, Brisbane, Australia; ${ }^{2}$ School of Psychology, University of Queensland, Brisbane, Australia; ${ }^{3}$ MRC Integrative Epidemiology Unit, School of Social and Community Medicine, University of Bristol, Bristol, UK; ${ }^{4}$ University of Queensland Diamantina Institute, Translational Research Institute, Brisbane, Australia; ${ }^{5}$ School of Social \& Community Medicine, University of Bristol, Oakfield House, Oakfield Grove, Bristol, UK;

${ }^{6}$ Max Planck Institute for Psycholinguistics, Nijmegen, The Netherlands; ${ }^{7}$ Department of Psychiatry, Washington University, St. Louis, Missouri, USA; ${ }^{8}$ Department of Psychology, La Trobe University, Melbourne, Australia;

${ }^{9}$ National Centre for Forensic Studies, Faculty of Education, Science, Technology and Mathematics, University of Canberra, Canberra, Australia; and ${ }^{10}$ Office of the Chief Forensic Scientist, Forensic Services Department, Victoria Police, Australia *Corresponding author e-mail: Yvonne.Ho@ qimrberghofer.edu.au ord.evans1@uq.edu.au

\section{SUPPLEMENTARY MATERIAL}

Supplementary material is linked to the online version of the paper at www.jidonline.org, and at http://dx.doi.org/10.1016/j.jid.2015.10.062.

\section{REFERENCES \\ Abecasis GR, Cherny SS, Cookson WO, et al. Merlin-rapid analysis of dense genetic maps using sparse gene flow trees. Nat Genet 2002; 30:97-101. \\ Boker S, Neale M, Maes H, et al. OpenMx 1.2 User Guide. Charlottesville, VA: Department of Psychology, University of Virginia; 2012. \\ Boyd A, Golding J, Macleod J, et al. Cohort pro- file: the "children of the 90s"-the index offspring of the Avon Longitudinal Study of Parents and Children. Int J Epidemiol 2013;42: $111-27$. \\ Holt S. The genetics of dermal ridges. Springfield, IL: Charles C. Thomas; 1968. \\ Li M, Gui H, Kwan J, et al. GATES: a rapid and powerful gene-based association test using extended Simes procedure. Am J Hum Genet 2011;88:283-93. \\ Machado J, Fernandes P, Roquetti R, et al. Digital dermatoglyphic heritability differences as evi- denced by a female twin study. Twin Res Hum Genet 2010;13:482-9. \\ Martin N, Eaves L, Loesch D. A genetical analysis of covariation between finger ridge counts. Ann Hum Biol 1982;9:539-52. \\ Medland S, Loesch D, Mdzewski B, et al. Linkage analysis of a model quantitative trait in humans: finger ridge count shows significant multivariate}

\section{Bacterial Sepsis Increases Survival in Metastatic Melanoma: Chlamydophila Pneumoniae Induces Macrophage Polarization and Tumor Regression}

Journal of Investigative Dermatology (2016) 136, 862-865; doi:10.1016/j.jid.2015.12.032

\section{TO THE EDITOR}

The initiative of this study was the unexpected complete tumor regression in a patient with stage IV cutaneous metastatic melanoma, who suffered multifactorial sepsis syndrome during BOLD (bleomycin, oncovin, lomustine, and dacarbazine) chemotherapy (see Supplementary Figure S1 online). After targeted antibiotic treatment and combined complication-free chemotherapy, the patient's physical condition improved, and the metastases unexpectedly disappeared. The patient has been asymptomatic and metastasisfree since the end of BOLD therapy. A significant decrease in the volume of the previously palpable axillary and abdominal metastases had already been observed when BOLD therapy was interrupted because of sepsis. A timeline of events is given in Table 1.

Molecular genetics research in the last decade assisted in the development of BRAF inhibitors and immunooncological agents, which resulted in
Abbreviations: BOLD, bleomycin, oncovin, lomustine, and dacarbazine; $C O X$, cyclooxygenase; $C P$, Chlamydophila pneumoniae; LM, lung metastasis

Accepted manuscript published online 29 December 2015; corrected proof published online 11 February 2016

(c) 2015 The Authors. Published by Elsevier, Inc. on behalf of the Society for Investigative Dermatology. linkage to 5q14.1. PLoS Genet 2007a;3: 1736-44.

Medland S, Park D, Loesch D, et al. Ridgecounter: a program for obtaining semi-automated finger ridge counts. Ann Hum Biol 2007b;34: 504-17.

Medland S, Zayats T, Glaser B, et al. A variant in LIN28B is associated with 2D:4D finger-length ratio, a putative retrospective biomarker of prenatal testosterone exposure. Am J Hum Genet 2010;86:519-25.

Mulvihill J, Smith D. The genesis of dermatoglyphics. J Pediatr 1969;75:579-89.

Penrose L. Memorandum on dermatoglyphic nomenclature, Birth Defects Original Article Series, vol. 4. NY: National Foundation-March of Dimes; 1968.

Willer C, Li Y, Abecasis G. METAL: fast and efficient meta-analysis of genomewide association scans. Bioinformatics 2010;26: 2190-1.

Yao J, Zhou B, Zhang J, et al. A new tumor suppressor LncRNA ADAMTS9-AS2 is regulated by DNMT1 and inhibits migration of glioma cells. Tumour Biol 2014;35:1-10.

Zhang J, Rubio V, Zheng S, et al. Knockdown of OLA1, a regulator of oxidative stress response, inhibits motility and invasion of breast cancer cells. J Zhejiang Univ Sci B 2009;10:796-804. significant improvement in the life expectancy of melanoma patients. Dacarbazine-based chemotherapies, once the gold standard (Hobohm, 2001; Wiemann and Starnes, 1994), are still approved and widely applied in melanoma therapy, but their efficacy is relatively low (Garbe et al., 2011). Therefore, the fact that clinical improvement was observed quite early during the chemotherapy suggested other factors behind the outcome, and the concurrent sepsis seemed to offer a potential explanation.

It has long been recognized that cancer patients might recover after bacterial infections (Hobohm, 2001). The hypothesis was that fever and tumor necrosis factor- $\alpha$ induced by the 


\section{Table 1. Timeline of clinical case report}

Day

-360 The patient herself detected a bleeding nevus-like lesion on the back and an enlarged axillary lymph node. No steps were taken.

120 Hospital visit. X-ray, mammography, and abdominal Doppler seem to be negative. Axillary lymph node biopsy was proposed. The patient was temporarily lost to follow-up.

$0 \quad$ Hospital visit for abdominal pain. Gastritis was diagnosed, and a gastric polyp was removed. Tumor masses were discovered in the retroperitoneal lymph nodes $(15-20 \mathrm{~mm})$, spleen $(67 \mathrm{~mm})$, and bladder $(40 \times 68 \mathrm{~mm})$. Another tumor was detected in the brain by computerized tomography $(40 \mathrm{~mm})$.

$4 \quad$ The intracranial tumor mass was removed surgically and diagnosed as amelanotic melanoma metastasis.

24 Cranial radiotherapy was initiated.

30 Leukocytosis, fever. Amoxicillin + clavulanic acid treatment.

32 Radiotherapy completed.

35 BOLD (bleomycin, oncovin, lomustine, and dacarbazine) chemotherapy initiated.

37 Chemotherapy was suspended on the third day because of vomiting and fever. Gastric fluid contained Escherichia coli and Candida albicans. Clostridium difficile toxin also was detected. Fluconazole and ceftriaxone (later metronidazole) treatment was initiated.

52 Central venous catheter was removed because of putative Pseudomonas aeruginosa infection, which was later confirmed by blood test.

59 The primary tumor was excised and analyzed (melanoma malignum, $\mathrm{Br} 1.52 \mathrm{~mm}$, C1. III., pT2b).

77 BOLD, second treatment cycle. Decrease of axillary and abdominal metastases was detected.

120 BOLD, third cycle. Further improvement of the axillary and intra-abdominal metastases was recorded. No intra-abdominal lymphadenomegaly was seen, and a single liver metastasis and shrinking splenic metastasis were detected.

162 BOLD, fourth cycle. Complete remission of the axillary and abdominal metastases was observed.

210 BOLD, fifth cycle. Complete remission of the axillary and abdominal metastases was observed.

255 BOLD, sixth cycle. The patient was asymptomatic, verified by positron emission tomography/computerized tomography to be metastases-free.

$>1,500$ The patient is asymptomatic, verified by positron emission tomography/computerized tomography to be metastases-free.

infectious agents caused tumor regression, but this could not be reproduced by administration of tumor necrosis factor- $\alpha$ or by hyperthermia (Nauts et al., 1946; Tsung and Norton, 2006). It has been observed that an attenuated form of Listeria monocytogenes can infect cancer cells but not normal cells, and this phenomenon resulted in a potentially effective experimental cancer therapy (Quispe-Tintaya et al., 2013).

Vaccination with intracellular pathogens such as bacille Calmette-Guérin or vaccinia virus significantly decreased the incidence of melanoma (Krone et al., 2005). However, a convincing explanation is still missing.

Although it is generally accepted that antitumor immune mechanisms overlap with antibacterial immune responses (Adams, 2009; Chen et al., 2007), the exact mechanism induced by microbes is not understood.
Because immune responses also appear to be decisive factors in the outcome of melanoma (Ridnour et al., 2013; Shimanovsky et al., 2013), we hypothesized that sepsis, by triggering polarized, "joint" antibacterial and antitumor immune responses, could induce tumor regression. This hypothesis was tested in our experimental model.

To clarify the role of the adaptive immune system in the antitumor immune mechanisms induced by Chlamydophila pneumoniae (CP; successfully identified in the primary melanoma after our patient recovered from sepsis; see Supplementary Figure S1f), lung metastases (LMs) were induced in immunocompetent $\mathrm{C} 57 \mathrm{BL} / 6$ mice or immunodeficient NSG mice. Animals were then $\mathrm{CP}$ - or mock-treated (see Supplementary Materials online). To assess the effects of treatment, histological, immunological, and molecular analyses were performed.
In immunocompetent, CP-treated animals, the number of LMs significantly decreased $(P=0.003)$ (Figure 1a) and survival (Figure 1t) significantly increased $(P=0.04)$ compared to mock treatment. This was not observed in immunodeficient mice, and the treated animals did not develop fever $\left(33.2{ }^{\circ} \mathrm{C} \pm 1.0{ }^{\circ} \mathrm{C}\right.$ mock vs. $34.8{ }^{\circ} \mathrm{C} \pm 0.5{ }^{\circ} \mathrm{C} \mathrm{CP}$ ) or high plasma levels of tumor necrosis factor- $\alpha$, which is against the "fever hypothesis" (Hobohm, 2001; Wiemann and Starnes, 1994).

Histological analysis of slices from the lungs of mock-treated melanomabearing immunocompetent mice showed a high number of LMs, with frequent intratumor necrosis (Figure 1e). In contrast, fewer and smaller foci of regressive LMs were observed in the CP-treated immunocompetent animals (Figure 1f). Moreover, in this group, high numbers of tumor-infiltrating mononuclear histiocytes and lymphoid cells were identified in the LMs. The LMs did not exhibit significant intratumor immune reactions in the immunodeficient mice, regardless of treatment type (Figure $1 \mathrm{~g}$ and h). Markedly increased immune reaction in the lungs of the $\mathrm{CP}$-treated mice was also verified by immunolabeling of the cell surface activation markers CD11b and CD80 (Figure 1i-I). Immune cell invasion was not detected after mock treatment; the immune cells were concentrated in the marginal zones of the tumors (Figure $1 \mathrm{i}$ and $\mathrm{k}$ ). In contrast, after CP treatment, marked infiltration by activated lymphocytes was seen in the internal tumor stroma (Figure $1 \mathrm{j}$ and $\mathrm{I}$ ), and the differences were significant $(P=0.0001)$ (Figure $1 \mathrm{~m}$ and $\mathrm{n}$ ).

To assess macrophage polarization, M1 (antitumor) or M2 (protumor) macrophage-specific cytokine and chemokine transcriptome profiling was performed (Mantovani et al., 2004). Macrophage markers were detected with quantitative PCR from pooled lung samples 2, 4, and 12 hours after mock or $\mathrm{CP}$ treatment. Four hours after $\mathrm{CP}$ application, markedly increased levels of M1-specific mRNA transcripts for CCL2, CCL3, IL-6, CXCL10, CCL7, CD80, CXCL11, CXCL9, IL-23, and tumor necrosis factor- $\alpha$ were detected. In line with this, mRNA expression of 
K Buzás et al.

C. Pneumoniae Increases Survival in Melanoma

$\mathrm{C} 57 \mathrm{BL} / 6$

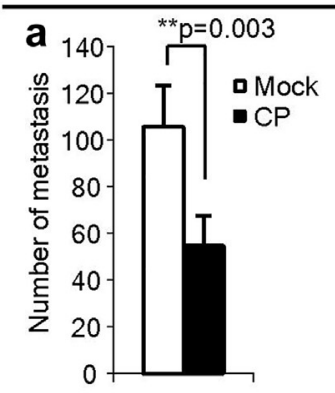

e
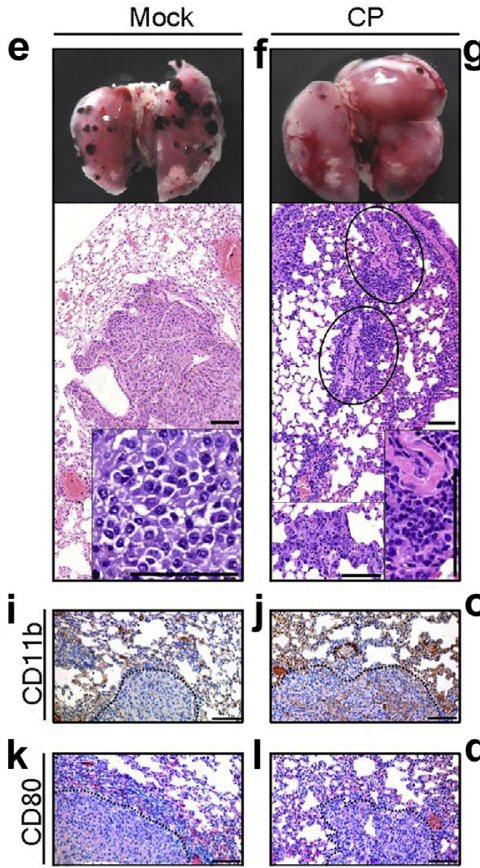
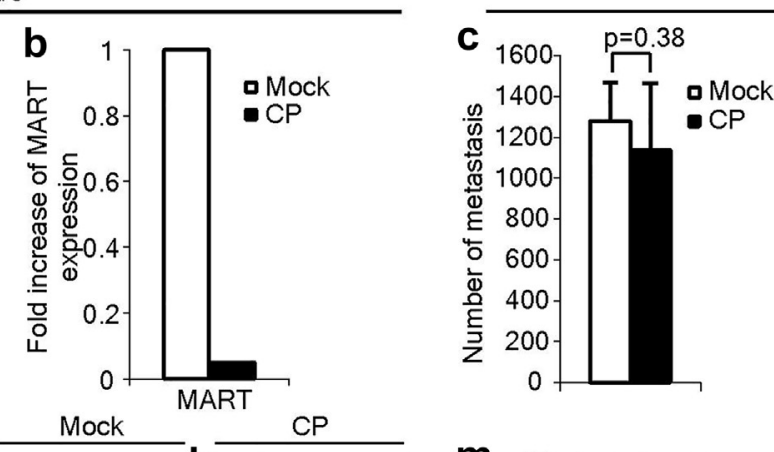

NSG
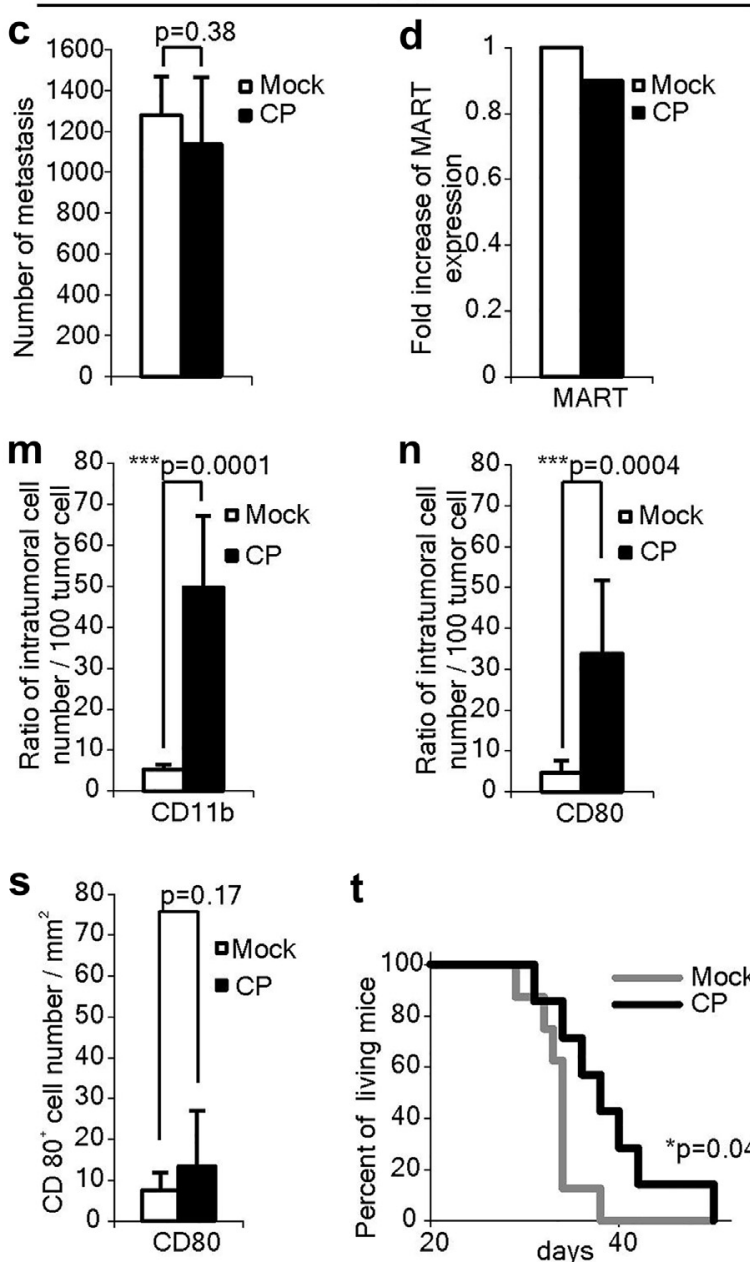

$\mathbf{t}$

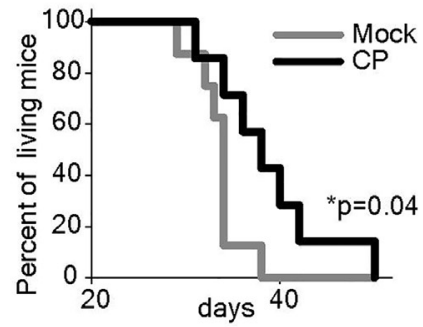

Figure 1. Chlamydophila pneumoniae treatment results in melanoma metastasis regression and increases survival of animals as well as of CD11 $b^{+}$and $\mathrm{CD}^{+}{ }^{+}$immune cell infiltration of tumor tissues. $(\mathbf{a}, \mathbf{c})$ Number of lung metastases, $(\mathbf{b}, \mathbf{d})$ melanoma antigen MART, and (t) survival rate of mock or Chlamydophila pneumoniae (CP)-treated immunocompetent C57BL/6 and immunodeficient NSG mice. (e-h) Representative images and hematoxylin and eosinstained histological sections of dissected lungs of (e) mock and (f) CP-treated immunocompetent mice, as well as of (g) mock and (h) CP-treated immunodeficient (NSG) animals. Note that subpleural metastasis formation is extensive in diameter but not in thickness in NSG mice (arrowheads). Bars $=100 \mu \mathrm{m}$. (e) Trophical necroses indicate high tumor burden. Inset: atypical tumor cells and regions of necrosis. (f) Circles and right inset: foci of regressive metastases. Left inset: areas of residual pneumonitis after CP treatment. ( $\mathbf{g}$, $\mathbf{h}$ ) In both mock and CP-treated NSG mice, miliary metastases were developed subpleurally (arrowheads) and intraparenchymally (circles) without significant inflammatory reactions (insets: higher magnification of intraparenchymal metastases). (i-l) Immunohistochemistry of (i, j) CD11 b (diaminobenzidine, brown) and (k, l) CD80 (Fast red, red) on lungs of (i, $\mathbf{k}$ ) mock or ( $\mathbf{j}, \mathbf{l})$ CP-treated C57BL/6 mice. Dashed lines indicate tumor border. (i-r) Arrows indicate infiltrating immune cells. Intratumoral number of $(\mathbf{m}) \mathrm{CD} 11 \mathrm{~b}^{+}$and $(\mathbf{n}) \mathrm{CD} 80^{+}$cells determined as a ratio of 100 tumor cells in $\mathrm{C} 57 \mathrm{BL} / 6 \mathrm{mice}$. (s) $\mathrm{CD}^{+} 0^{+}$cells in NSG lungs counted by square millimeter. Data are given as mean \pm standard deviation.

most M2-specific markers decreased. Interestingly, the levels of some important M2 markers (CXCL13, IL1Ra) were actually increased (see Supplementary Figure S2a and b online). Upon CP administration, the quantity of M1specific cytokine and chemokine mRNA was significantly increased $(P=$ $0.014)$ after 4 hours in comparison to M2 markers.

Alteration in the expression pattern of cyclooxygenase (COX)- 1 and COX-2 is one of the key markers of macrophage polarization (Mantovani et al., 2013; Martinez et al., 2006). Western blot analysis revealed that 12 hours after CP treatment, protein expression of the M2-specific COX-1 decreased by half, whereas protein expression of the M1specific COX-2 increased more than twofold (see Supplementary Figure S2C and d).

Two hours after $\mathrm{CP}$ treatment but not after mock treatment, CXCL1 melanoma growth factor immunoreactivity become undetectable (see Supplementary Figure S3a and b online). To assess whether this in vivo phenomenon was due to a direct $\mathrm{CP}$ CXCL1 interaction, equal amounts of recombinant CXCL1 were incubated in vitro (in the presence of protease inhibitors) with increasing quantities of CP. CXCL1 levels were determined by western blotting. CP depleted CXCL1 in a dose-dependent manner, suggesting strong and direct binding by $\mathrm{CP}$ (see Supplementary Figure S3c and d).

Our results seem to indicate that CP treatment does indeed induce a complex antitumor response. We showed that $\mathrm{CP}$ treatment can suppress LM formation in immunocompetent (but not in immunodeficient) mice. M1type macrophage polarization was 
demonstrated, which is associated with antitumor effects (Sica et al., 2008). The antitumor immune polarization/ activation was further supported by the profound enrichment of CD80- and CD11b-expressing immune cells in the lungs of CP-treated animals (Prebeck et al., 2001). Of special importance, the melanoma growth factor CXCL1 was completely depleted by $\mathrm{CP}$, both in vivo and in vitro.

Because (i) CXCL1-induced NF-KB activity was shown to facilitate melanoma transformation by allowing melanocytes to escape apoptosis and (ii) $\quad \mathrm{\kappa} \mathrm{B}-\alpha \quad \Delta \mathrm{N}$ (super-repressor of NF-rkB) reduced tumor growth and metastatic potential of melanoma cells (Dhawan et al., 2002), we consider the possible scenario not only that $\mathrm{CP}$ induced M1-type macrophage polarization but that CXCL1-induced depletion could significantly contribute to tumor regression. Evidently, results of the animal study strongly support our assumption about the role of sepsis in the observed outcome; however, these data cannot exclude the role of the BOLD therapy. The conclusion that can be safely drawn at this point is that sepsis, in the context of BOLD therapy, resulted in dramatic improvement, otherwise not seen in uncomplicated therapy, which suggests that the occurrence of sepsis was an event of key importance.

\section{CONFLICT OF INTEREST}

The authors state no conflict of interest.

\section{ACKNOWLEDGMENTS}

This work was approved by The Medical Ethics Committee of University of Szeged and was conducted in accordance with the Declaration of Helsinki Principles. Written informed consent was obtained from our patient. This project has been funded by Hungarian Scientific Research FundOTKA PD 84064, OTKA 112493, TAMOP-4.2.2A-11/1/KONV-2012-0025, IPA HUSRB/1203/ 214/230. We thank Zoltan Kis and Laszlo Puskas for PCR, Gabriella Dobra for technical assistance; Erno Duda, Janos Minarovits, and Gabor
Braunitzer for helpful discussions; and Biocenter Ltd for the RNA purification kit.

Krisztina Buzás ${ }^{1,2,9, *}$,

Annamária Marton ${ }^{2,9}$, Csaba Vizler ${ }^{2}$, Edina Gyukity-Sebestyén ${ }^{2}$,

Mária Harmati ${ }^{2}$, Katalin Nagy ${ }^{1}$, Ágnes Zvara ${ }^{3}$, Róbert L. Katona ${ }^{3}$, Vilmos Tubak ${ }^{4}$, Valéria Endrész ${ }^{5}$, István B. Németh ${ }^{6}$, Judit Oláh ${ }^{6}$, László Vígh ${ }^{2}$, Tamás Bíró ${ }^{7}$ and Lajos Kemény ${ }^{6,8}$

${ }^{1}$ University of Szeged, Faculty of Dentistry, Szeged, Hungary; ${ }^{2}$ Hungarian Academy of Sciences, Biological Research Centre, Institute of Biochemistry, Szeged, Hungary; ${ }^{3}$ Hungarian Academy of Sciences, Biological Research Centre, Institute of Genetics, Szeged, Hungary; ${ }^{4}$ Creative Laboratory Ltd, Szeged, Hungary; ${ }^{5}$ Department of Medical Microbiology and Immunobiology, University of Szeged, Szeged, Hungary; ${ }^{6}$ Department of Dermatology and Allergology, University of Szeged, Szeged, Hungary; "DE-MTA "Lendület" Cellular Physiology Research Group, Departments of Immunology and Physiology, University of Debrecen, Medical Faculty, Debrecen, Hungary; and ${ }^{8}$ MTA-SZTE Dermatological Research Group, University of Szeged, Szeged, Hungary

${ }^{9}$ These authors contributed equally to this work. *Corresponding author e-mail: kr.buzas@ gmail.comorbuzask@brc.hu

\section{SUPPLEMENTARY MATERIAL}

Supplementary material is linked to the online version of the paper at www.jidonline.org, and at http://dx.doi.org/10.1016/j.jid.2015.12.032.

\section{REFERENCES}

Adams S. Toll-like receptor agonists in cancer therapy. Immunotherapy 2009;1:949-64.

Chen K, Huang J, Gong W, Iribarren P, Dunlop NM, Wang JM. Toll-like receptors in inflammation, infection and cancer. Int Immunopharmacol 2007;7:1271-85.

Dhawan P, Richmond A. Role of CXCL1 in tumorigenesis of melanoma. J Leukoc Biol $2002 ; 72: 9-18$.

Garbe C, Eigentler TK, Keilholz U, Hauschild A, Kirkwood JM. Systematic review of medical treatment in melanoma: current status and future prospects. Oncologist 2011;16:5-24.

Hobohm U. Fever and cancer in perspective. Cancer Immunol Immunother 2001;50:391-6.
Krone B, Kolmel KF, Henz BM, Grange JM. Protection against melanoma by vaccination with Bacille Calmette-Guerin (BCG) and/or vaccinia: an epidemiology-based hypothesis on the nature of a melanoma risk factor and its immunological control. Eur J Cancer 2005;41: 104-17.

Mantovani A, Biswas SK, Galdiero MR, Sica A, Locati M. Macrophage plasticity and polarization in tissue repair and remodelling. J Pathol 2013;229:176-85.

Mantovani A, Sica A, Sozzani S, Allavena P, Vecchi A, Locati M. The chemokine system in diverse forms of macrophage activation and polarization. Trends Immunol 2004;25: 677-86.

Martinez FO, Gordon S, Locati M, Mantovani A. Transcriptional profiling of the human monocyte-to-macrophage differentiation and polarization: new molecules and patterns of gene expression. J Immunol 2006;177: 7303-11.

Nauts HC, Swift WE, Coley BL. The treatment of malignant tumors by bacterial toxins as developed by the late William B. Coley, M.D., reviewed in the light of modern research. Cancer Res 1946;6:205-16.

Prebeck S, Kirschning C, Dürr S, da Costa C, Donath B, Brand K, et al. Predominant role of toll-like receptor 2 versus 4 in Chlamydia pneumoniae-induced activation of dendritic cells. J Immunol 2001;167:3316-23.

Quispe-Tintaya W, Chandra D, Jahangir A, Harris M, Casadevall A, Dadachova E, et al. Nontoxic radioactive Listeria(at) is a highly effective therapy against metastatic pancreatic cancer. Proc Natl Acad Sci U S A 2013;110: 8668-73.

Ridnour LA, Cheng RY, Switzer $\mathrm{CH}$, Heinecke JL, Ambs S, Glynn S, et al. Molecular pathways: toll-like receptors in the tumor microenvironment-poor prognosis or new therapeutic opportunity. Clin Cancer Res 2013;19:1340-6.

Shimanovsky A, Jethava A, Dasanu CA. Immune alterations in malignant melanoma and current immunotherapy concepts. Expert Opin Biol Ther 2013;13:1413-27.

Sica A, Larghi P, Mancino A, Rubino L, Porta C, Totaro MG, et al. Macrophage polarization in tumour progression. Semin Cancer Biol 2008; 18:349-55.

Tsung K, Norton JA. Lessons from Coley's toxin. Surg Oncol 2006;15:25-8.

Wiemann B, Starnes CO. Coley's toxins, tumor necrosis factor and cancer research: a historical perspective. Pharmacol Ther 1994;64: 529-64. 